


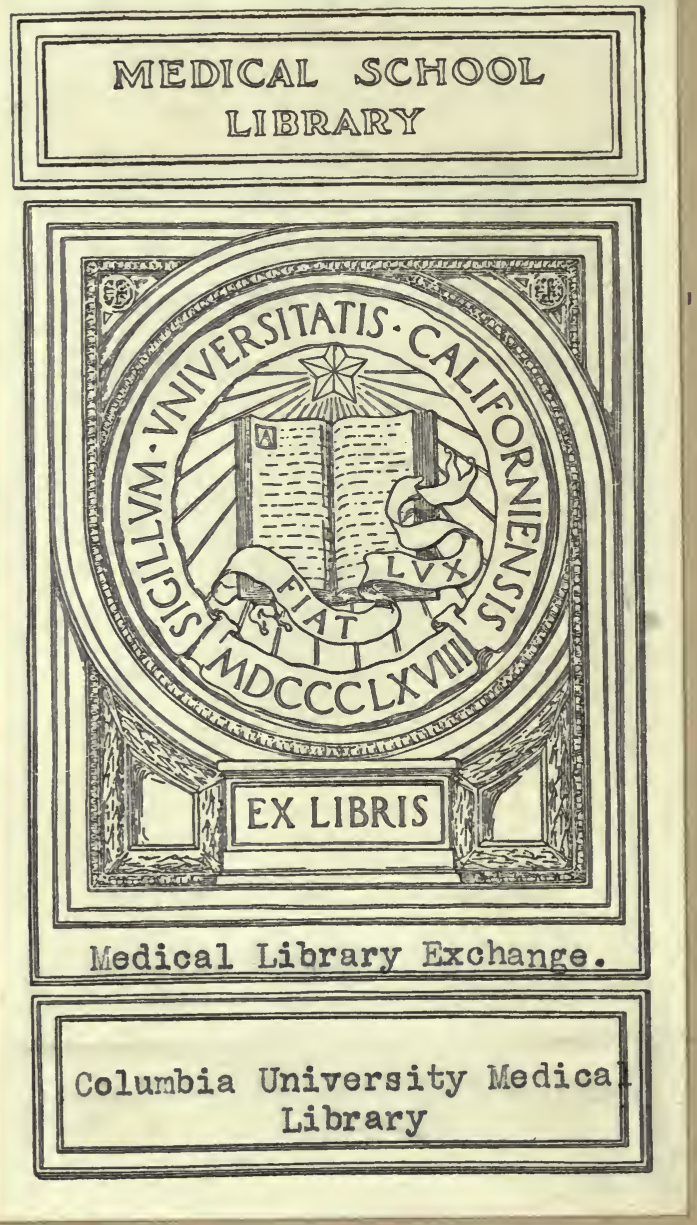




Digitized by the Internet Archive in 2007 with funding from Microsoft Corporation 



\title{
ADVANCED LESSONS
}

IN

\section{Practical Physiology FOR}

\author{
STUDENTS OF MEDICINE
}

\section{By \\ RUSSELL ЦBURTON-OPITZ \\ S. M., M. D., Рн. D.}

Associate Professor of Physiology, Columbia University; Professorial Lecturer in Physiology in Teachers College and the Extension Department of Columbia University

\section{ILLUSTRATED}

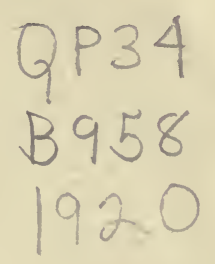 \\ QP34 B958 1920}

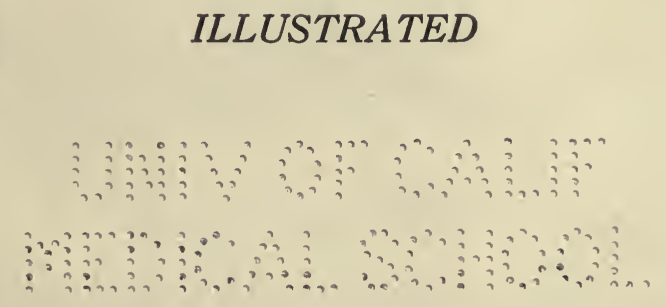

PHILADELPHIA AND LONDON

W. B. SAUNDERS COMPANY 1920 


\section{Mencoma

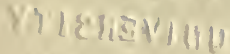 Xiation}

\title{
Copyright, 1920, by W. B. Saunders Company
}

\author{
$\longrightarrow$
}




\section{CONTENTS}

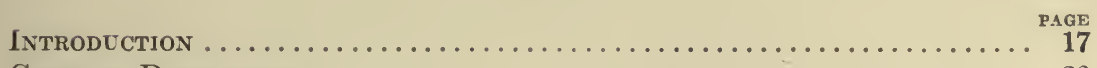

General Directions .................................. 20

Muscle and Nerve .................................... 21

Ameboid and Ciliary Motion. Methods of Stimulation............ 21

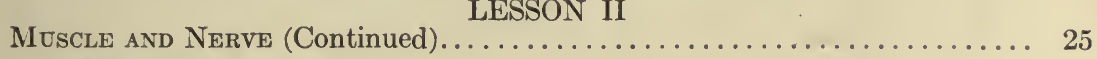

Myography $\ldots \ldots \ldots \ldots \ldots \ldots \ldots \ldots \ldots \ldots \ldots \ldots \ldots \ldots \ldots \ldots \ldots \ldots \ldots, 25$

Muscle and Nerve (Continued)........................... 35

Irritability, Conductivity, and Elasticity of Muscle. The Power of Muscle

Tissue in Relation to the Cross-section and Arrangement of Its Fibers

\section{LESSON IV}

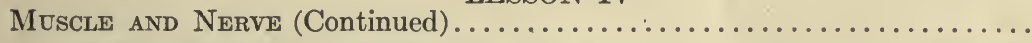

Single Contraction, Summation and Fusion of Contractions. Tetanus. Influience of Changes in the Strength of the Stimulus and Load of the Muscle .............................................

MUSCLe ANd Nerve (Continued)....... LESSON V

Influence of Temperature, Chemicals, and Fatigue Upon the Contraction of Muscle ...................................................

\section{LESSON VI}

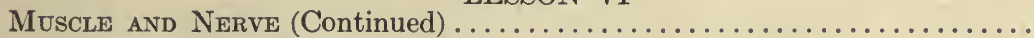

Contraction of Human Muscle. Influence of Blood-supply. Smooth Muscle.........................................

LESSON VII

Muscle and Nerve (Continued)

.............................

Speed of the Nerve Impulse in the Frog and Man. Conduction in Both Directions.

Muscle and Nerve (Continued)

\section{LESSON VIII}

Conduction in Nerve. Action Current of Muscle and Nerve. Stimulation of Motor Points.........................................

\section{LESSON IX}

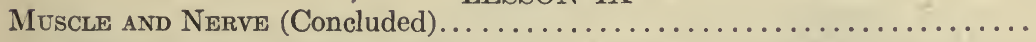

Electrotonus. Cathodic and Anodic Excitation. Law of Unipolar Stimulation of Human Muscle and Nerve.

\section{LESSON $\mathrm{X}$}

The BLOOD.

The Coagulation of the Blood. Counting of the Blood-corpuscles........ 


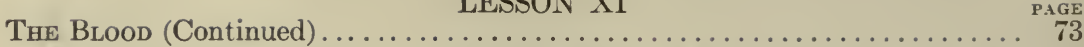

The Counting of Human Blood-corpuscles. Specific Gravity and Appearance of Blood

LESSON XII

THE BLood (Concluded) . . . . . . . . . . . . . . . . . . . . . 77

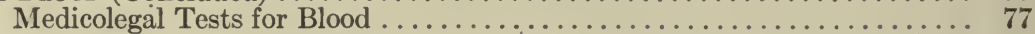

LESSON XIII

The Heart . . . . . . . . . . . . . . . . . . . . . . . . . . . . 79

Registration of the Heart-beat. Refractory Period. Extrasystole. Excised Heart. Action of Strips of Ventricular Tissue..............

LESSON XIV

The Heart (Continued) . . . . . . . . . . . . . . . . . . . . . . .

Inhibition and Acceleration of the Simple Heart. Action of Nicotin,

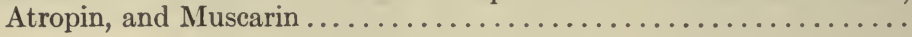

\section{LESSON XV}

The Heart (Continued) . . . . . . . . . . . . . . . . . . . . . . 89

Stannius' Experiment. Staircase Phenomenon. Summation of Stimuli. Action of the Constant Current, Ether, and Chloroform. Dissection

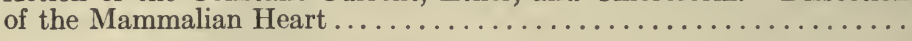

LESSON XVI

The Heart (Continued) . . . . . . . . . . . . . . . . . . . . . . 91

The Beating Mammalian Heart. Heart-block. Fibrillation.......... 91

Percussion and Auscultation of the Human Heart Under Different Con-

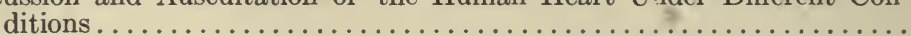

\section{LESSON XVIII}

The Circulation..................................... 99

The Capillary Circulation. Conversion of an Intermittent Into a Constant Flow. Schema of the Circulation................... 99

LESSON XIX

The Circulation (Continued).................................. 103

The Cause and Velocity of the Pulse. Direct Method of Ascertaining the

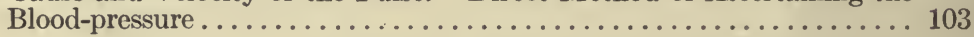

\section{LESSON XX}

The Circulation (Continued) . . . . . . . . . . . . . . . . . . . . . . 109

Venous Valves. Influence of Dyspnea Upon the Blood-pressure. Action of Amyl Nitrite and Ardenalin. Hemorrhage................ 109

\section{LESSON XXI}

The Circulation (Continued) ................................. 113

The Effect of Division and Stimulation of the Vagus Nerve Upon the Bloodpressure and Action of the Heart....................... 113

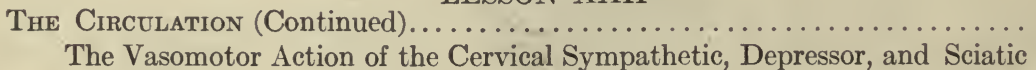


LESSON XXIII
The Circulation (Continued) $\ldots \ldots \ldots \ldots \ldots \ldots \ldots \ldots \ldots \ldots \ldots \ldots \ldots \ldots \ldots$
121

The Vasomotor Action of the Greater Splanchnic Nerve. The Vascularity

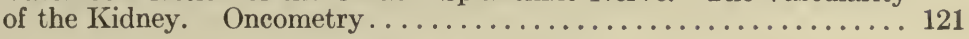

\section{LESSON XXIV}

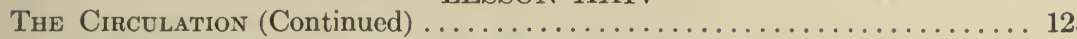

The Indirect Method of Measuring Blood-pressure. Effect of Posture and

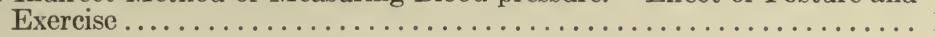

The Circulation (Concluded) LESSON XXV

The Character and Velocity of the Arterial and Venous Pulsations. Polyg-

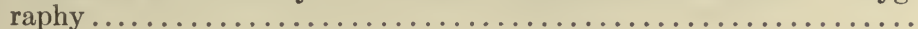

\section{LESSON XXVI}

Respiration ......................................... 133

Mechanics of Respiration .......................... 133

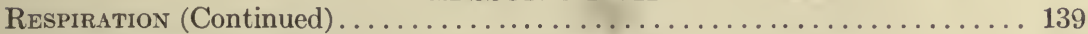

Stethography. Methods of Artificial Respiration. Pulmotor......... 139

Respiration (Continued) ........

\section{LESSON XXVIII}

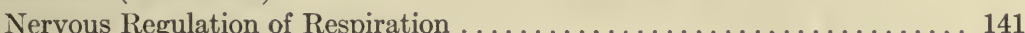

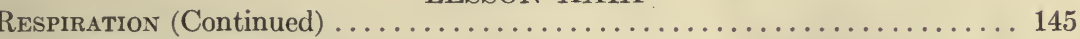

Localization of the Respiratory Center. Placenta. Respiration in the

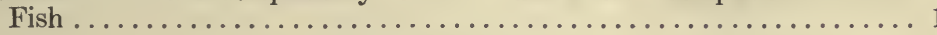

Respiration (Continued)

\section{LESSON XXX}

The Circulation in the Lung of the Frog. Phenomena of Inflammation. Effect of Changes in Intrathoracic Pressure Upon the Lesser Circuit . 147

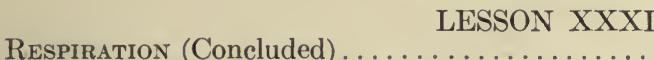

Respiration (Concluded)

Elimination of Carbon Dioxid and Consumption of Oxygen

LESSON XXXII

The Nervous System

Reflex Action

\section{LESSON XXXIII}

The Nervous System (Continued).

Reflex Action. Removal of Cerebrum .

\section{LESSON XXXIV}

The Nervods System (Continued)

Stimulation of the Cerebrum. The Function of the Roots of the Spinal Cord

The Nervous System (Concluded)

\section{LESSON XXXV}

Reaction Time

\section{LESSON XXXVI}

The Sense Organs . .....................
Cutaneous and Muscular Sensations 
The Sense Organs (Continued) . . . . . . . . . . . . . . . . . . 173

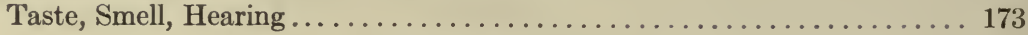

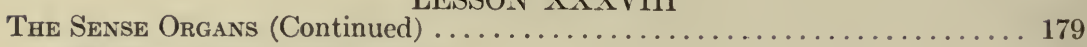

The Static and Dynamic Senses....................... 179

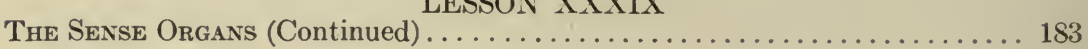

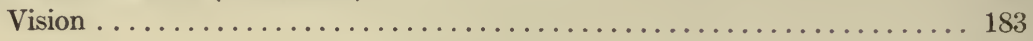

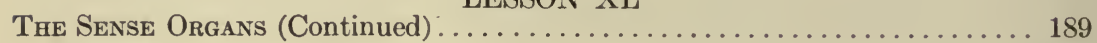

Vision . . . . . . . . . . . . . . . . . . . . . . . . . . . 189

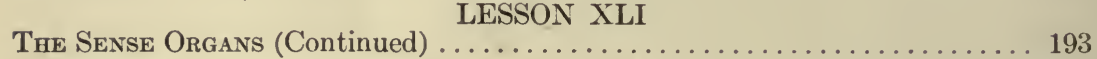

Vision . . . . . . . . . . . . . . . . . . . . . . . . . . . 193

The Sense Organs (Concluded) . . . . . . . . . . . . . . . . . . . 199

Vision . . . . . . . . . . . . . . . . . . . . . . . . . . . 199

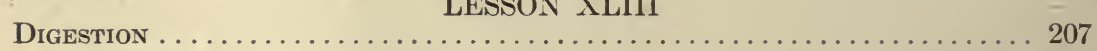

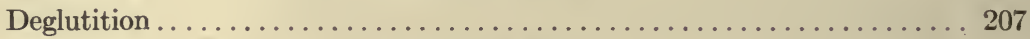

Digestion (Continued) . . . . . . . . . . . . . . . . . . . . . . 209

Secretion of Saliva .............................. 209

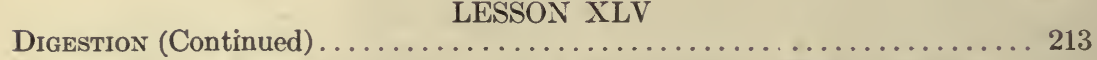

- Secretion of Pancreatic Juice. Action of Secretion. Gastro-enterostomy. 213

LESSON XLVI

Digestion (Concluded) . . . . . . . . . . . . . . . . . . . . 215

Lacteals and Thoracic Duct. Peristalsis. Secretion of Bile......... 215

LESSON XLVII

Absorption . . . . . . . . . . . . . . . . . .

Osmosis. Intestinal Peristalsis, Secretion of Intestinal Juice. Absorption From the Small Intestine ............................

LESSON XLVIII

Excretion . . . . . . . . . . . . . . . . . . . . . . . . . . . . 221

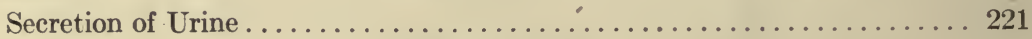

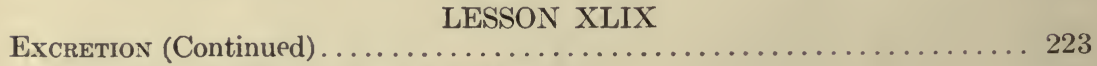

Secretion of Sweat. Body Temperature.................. 223

LESSON L

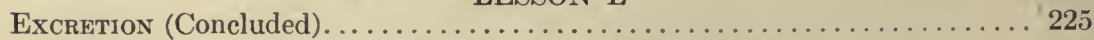

The Innervation of the Bladder. Pilomotor Reactions ............ 225

Demonstrations to be Given in Connection with the Preceding Lessons. 227

Weights and Measures ................................. 229

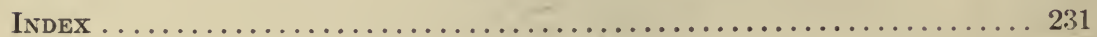




\section{ADVANCED LESSONS IN PRACTICAL PHYSIOLOGY}

\section{INTRODUCTION}

IN order to prepare the medical student for the clinical work that is to follow during his subsequent years of study, the subject of physiology must be presented to him in an eminently practical manner. For this reason the purely didactic lectures of not so many years ago have gradually been displaced by more or less informal discourses between the lecturer and students, pertaining more particularly to topics of unusual complexity and clinical value. Furthermore, the experiments which formerly constituted a large part of the physiologic lecture, have since been separated from the purely didactic subject matter and have been combined into a continuous course of practical exercises and demonstrations.

The earlier "experimental lectures" have failed in their purpose, because much time was frequently wasted in overcoming technical difficulties not apparent at the beginning of the hour, and because little opportunity was afforded the students to become acquainted with the apparatus and the technic required to perform physiologic experiments. These difficulties have been met in large part by instituting a course in practical physiology, designed so that the student himself may perform simple and instructive experiments. Obviously, the acquisition of knowledge by the laboratory method consumes a longer period of time and requires a definite experimental aptitude on the part of the student. Furthermore, this method of teaching entails the expenditure of large sums of money for apparatus and the salaries of additional teachers. These difficulties, however, have been overcome in recent years in all the schools of higher grade, and practical courses in physiology are now an accomplished fact, and rightly so, because the benefits which the students derive from work of this kind cannot be overestimated. It cultivates the faculty of close observation and accurate rating of facts. It develops the power of logical thought and expression, and impresses upon them facts and principles otherwise scarcely noted and comprehended. Indeed, many students must see things in order to be able to obtain a clear mental picture of them, but when once seen, the impression is lasting. Where else than in medicine could this manner of teaching be of greater service?

Quite aside from the fact that this method constitutes an admirable means of imparting physiologic knowledge, it also enables the students 
to familiarize themselves with the use of operative instruments and the action of different drugs. During a course of this kind each student is repeatedly called upon to attend to the narcosis; to perform tracheotomy; to expose and ligate blood-vessels, and to isolate nerves and other structures. It need scarcely be emphasized that the operative technic acquired by him upon animals under test conditions, will serve him in good stead later on when forced to repeat these procedures upon human beings.

The contention that students may derive their knowledge of physiology wholly from practical work, is scarcely worthy of consideration. While the average student is well able to abstract definite single facts from experiments, he is as yet in no position to appraise these facts and to combine them into a connected story of physiologic events. Knowledge gained by experimentation alone is, indeed, very fragmentary. It is the duty of the lecturer to bridge over these defects and to supply the student with those fundamental data which he is to make use of later on in formulating physiologic principles. Facts, as such, are of little value unless they can be joined to yield certain truths which have a direct bearing upon the student's subsequent clinical work. The student should be made to "physiologize" along lines more closely related to his chosen profession and should attain this state in as short a time as possible. In many instances this mental evolution may be greatly facilitated by referring to problems of general interest, such as may be obtained from treatises upon comparative physiology, biology, physics, and chemistry. Comparative physiology, in particular, is very rich in facts which will greatly aid the lecturer in clearing up doubtful or complex points in special physiology.

In the medical schools of higher grade about three hundred hours are allotted to physiology, exclusive of physiologic chemistry and clinical physiology or experimental medicine. This period of time is spent in part in the laboratory and in part in the lecture room. As a rule, one hundred and eighty hours are assigned to practical work and one hundred and twenty hours to lectures and conferences. Inasmuch as the academic year usually comprises thirty weeks, exclusive of the time set aside for examinations, the above enumeration leads us to infer that each student must devote ten hours per week to physiology. In many institutions, however, the "concentrated" system of teaching is employed, enabling the student by constant daily attendance to complete his work in physiology within about four months-similar periods of time being set aside for anatomy and physiologic chemistry.

Before submitting these lessons in practical physiology to the students I should like to mention that I have attempted to embody in them all those experiments which can be conveniently performed with the aid of simple apparatus. The lessons begin with experiments upon muscle and nerve, and gradually make a greater and greater demand upon the experimental aptitude of the student. Those experiments which require complex apparatus and may be more conveniently 
displayed to a large number of students, have been embodied in the demonstrations. A brief list of experiments of this kind is given on pages 227, 228.

While it is difficult to perform physiologic experiments in accordance with a definite time-schedule, the material embodied in this book has been arranged in such a way that each lesson will require about three hours for its completion and each demonstration one hour, making a total of one hundred and eighty hours. At the end of each period a few minutes should be set aside for a review of the work performed. Special attention should at this time be paid to those students who have failed to observe and formulate the essential facts and principles to be derived from any particular lesson. It would be a pedagogically unsound principle to call the attention of the students to these facts beforehand, because introductory explanations tend to rob the student of the pleasure of independent investigation and thought. If the work in physiology is well balanced and co-ordinated between the class-room and the laboratory, preliminary talks pertaining to the general bearing of the different experiments are actually worse than useless. Such discussions should concern themselves more particularly with matters closely related to the methods and apparatus, so that the student may be in a more favorable position to avoid mistakes in his technic. I have endeavored to aid him in this regard by supplying him with this laboratory guide, amplified, for the reason just stated, with explanations bearing directly upon the experiments.

Columbia University,

R. Burton-Opitz. May, 1920. 


\section{GENERAL DIRECTIONS}

EACH class of students should be divided into sections of not more than 40 each. To each section should be allotted 4 assistants, so that each assistant may be held responsible for the work of 10 students, arranged in pairs. A larger number than this cannot well be attended to by one instructor.

When mammals are being used, as many as 8 students may be assigned to one operating table. The formation of larger groups is not to be recommended, because it lessens the chances of the individual student to perform a considerable part of the work himself. Neither does it seem advisable to decrease this number materially, owing to the fact that such reduction would necessitate an extra expenditure for apparatus and material which is not proportional to the increase in the efficiency of the teaching. Since the work of the students requires close supervision, one assistant should be assigned to each operating table. If an additional table is put in use, these students should receive the necessary attention from the instructors at the two neighboring tables.

The function of the instructor is to advise the students how to proceed, and to guide them by pertinent questions and practical hints through the work assigned to them. Nothing shculd be told the students which they can readily discover for themselves, and nothing should be done for them which they can conveniently do themselves. At least, this plan of teaching should be followed as soon as the students have received their first instruction in etherization, in performing tracheotomy, and in exposing different blood-vessels. A record should be kept of the work done by each student during every exercise, so that a different task may be assigned to him during the succeeding exercise.

Each student should make brief entries in his note-book pertaining to the results and bearing of the experiments performed by him. In addition, this book should contain diagrams of the apparatus, explanatory schemas, and the curves recorded by him in the course of these practical exercises.

Each pair of students should be in possession of a set of operating instruments, embracing two pairs of forceps, two pairs of scissors of different size, and two scalpels of different size. Ligatures, sponges, ether, and towels will be supplied by the attendant. A rubber apron should be worn by every student during the experiments upon mammals. Dissecting gowns and instruments are not regarded with favor in the physiologic laboratory.

At the end of each session the apparatus is to be taken apart and each piece carefully cleaned. Special receptacles are provided for the discarded organic material. 


\section{LESSON I \\ MUSCLE AND NERVE}

\section{AMEBOID AND CILIARY MOTION. METHODS OF STIMULATION}

1. Ameboid Motion.-Place a few drops of a hay infusion upon a glass slide. Bring a large and active ameba into the field of the microscope. Observe carefully the behavior and position of the organism, making drawings of its shape at regular intervals. Add a few granules of India-ink to the medium and observe how these particles are engulfed.

2. Protoplasmic Streaming.-Examine with the low power of a microscope a leaflet of a fresh specimen of nitella. Observe the movement of the protoplasm. What part of the cell is in motion? In which direction does the flow take place? How is the movement changed by mechanical stimuli? Note the effect of warmth upon the movement.

3. Ciliary Motion.--Etherize a frog and destroy its brain and spinal cord. Place the animal upon its back. Make a median incision through the lower jaw, and retract the segments laterally. Place a small piece of cork upon the mucous surface between the eyes. Moisten the surface with normal saline solution, if necessary. Note how the piece of cork is gradually carried by the action of the cilia toward the esophagus. Determine its rate of movement. Moisten the surface with normal saline solution the temperature of which has been raised 2 or 3 degrees above that of the room. Note the effect upon the movement of the cork.

Tilt the plate upon which the frog is resting until the cilia are no longer able to move the piece of cork. Hold the plate horizontally and put small bits of lead upon the cork until one is found which the cilia are unable to propel. What is your idea regarding the strength of these structures?

Place a small segment of the gill-plate of a fresh clam upon a slide for microsicopic observation. Straighten its edge and immerse the entire preparation in a few drops of the fluid obtained when the shell of the clam was opened. Study carefully the action of the cilia at intervals, and especially later on, when their movement has been considerably slowed so that single cilia may be clearly made out. What is the position of the cilia when at rest and when contracting? Apply normal saline solution which has been slightly heated $\left(25^{\circ} \mathrm{C}\right.$.). Note its effect upon the rapidity of the movement.

4. Structure of the Different Types of Muscle Tissue.-Unless still quite familiar with the general structure of non-striated, striated, and cardiac muscle, examine preparations of these tissues under the microscope. Study the action of the myoids in stentor or vorticella. 
5. Muscular Movement.-With a small blunt instrument scrape off the wing muscles of a beetle (hydrophilus) and place them upon a slide for microscopic study. Observe the waves of contraction passing over the individual muscle-fibers. Note the increase in the diameter of the fiber at the point of contraction.

6. Stimuli. Muscle-nerve Preparation.-Living substance possesses the properties of irritability, conductivity, and contractility, i. e., it is capable of receiving a stimulus, of conducting it to some other part of its substance, and of reacting toward it in accordance with its structural peculiarities. A stimulus is any extraordinary change in the

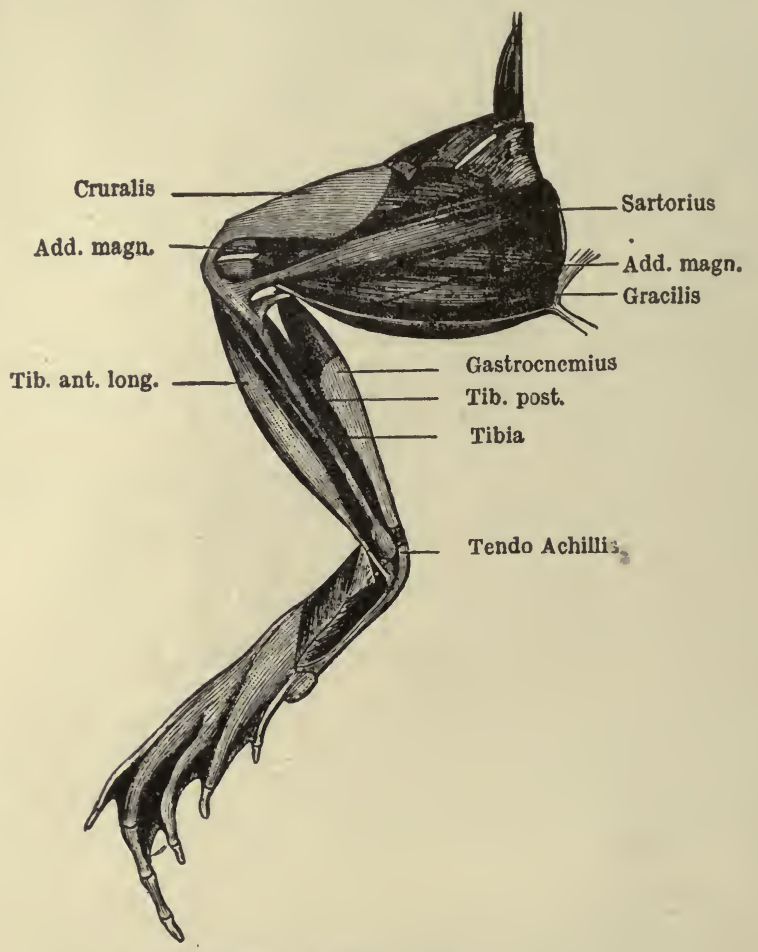

Fig. 1.-Muscles of Hind Leg of Frog. (Ecker.)

environment of the living entity, in consequence of which it evolves some form of energy. In this way a muscle may be made to contract and a gland to secrete. As a matter of convenience we usually employ the former tissue and principally that derived from the frog.

Grasp the pelvis of a lightly etherized frog between the thumb and index-finger of your left hand, allowing the ventral aspect of the thorax and head to rest upon your third and fourth fingers. With your right hand move the point of a small scalpel rapidly backward from between the eyes until you feel a depression at the junction of the head with the spinal column. This depression lies at the atlo-axoid articulation. 
Gently press the point of the scalpel transversely into this opening, dividing the spinal cord. Introduce a seeker through the incision, and, passing it upward into the cranial cavity, quickly destroy the cerebrum. Reverse the direction of the seeker and pass it downward through the vertebral canal, destroying the cord. This operation should consume only a few seconds of time. It is known as pithing, and corresponds to the destruction of the spinal cord of mammals by the excessive flexion of the head upon the spinal column. The odontoid process of the axis then lacerates the cord.

Amputate one leg. Remove the skin from the thigh and isolate the sciatic nerve. Cut away the muscles of the thigh, but preserve the femur and nerve. Suspend the leg from a clamp fastened horizontally to an iron stand by fixing the femur in its screw clip. Attach the central end of the sciatic nerve to a needle-holder and moisten the preparation with an isotonic solution of sodium chlorid (0.7 per cent.).

7. Direct and Indirect Stimulation of Muscle.-Use the following means to cause a contraction of the calf muscle (gastrocnemius):

(a) Mechanical stimulation. Pinch the end of the nerve with the forceps.

(b) Chemical stimulation. Dip the end of the nerve in a strong solution of sodium chlorid. Cut away the piece of the nerve used.

(c) Thermal stimulation. Heat a piece of wire and bring it in contact with the nerve.

(d) Electric stimulation. Apply the electrodes from the secondary coil of an inductorium to the nerve. Close, and open the key inserted in the primary circuit.

(e) Photic stimulation. Under these conditions rays of light do not serve as a stimulus.

Place your index-finger under the sole of the foot and repeat the electric stimulation. Explain the action of the gastrocnemius muscle. Remove the skin. Stimulate the muscle directly. Which stimulation is more effective?

Locate the tendo achillis. Carefully dissect the other leg and identify the sartorius and gracilis muscles. Open the abdomen of the frog and trace the sciatic nerve to its point of origin from the spinal cord. 





\section{LESSON II}

\section{MUSCLE AND NERVE (Continued)}

\section{MYOGRAPHY}

1. The Construction and Action of the Dry Cell.-The electric method of stimulation is employed most frequently in the laboratory because it is the most convenient. The electric energy is derived, as a rule, from a Voltaic cell. As a generator may be employed a Daniell, Grove, or Leclanché cell. The moist cells, however, have been displaced in the course of time by the so-called dry cells which give off no fumes and acids, need no refilling, and give, as a rule, good service at slighter cost. The dry cell commonly used is a modification of the Leclanché cell. It consists of a jacket of zinc lined with plaster of Paris and saturated with ammonium chlorid. Its inner space is taken up by a carbon plate which is surrounded by black oxid of manganese. The plate of carbon projecting from this mixture forms the positive pole or anode, whereas the negative pole or cathode is represented by the zinc.

Electricity "flows" from a place of high potential to a place of low potential. Hence, if the carbon and zinc of the battery are connected by means of a conductor, say, a copper wire, a current is set up which leaves the generator at the former pole and enters it at the latter. Inside the cell the current flows from the zinc to the carbon to complete the circuit (Fig. 2).

The difference in the potential between the two poles of a battery constitutes the electromotive force. It is maintained by the interaction of the chemical substances contained in the battery. The latter, therefore,

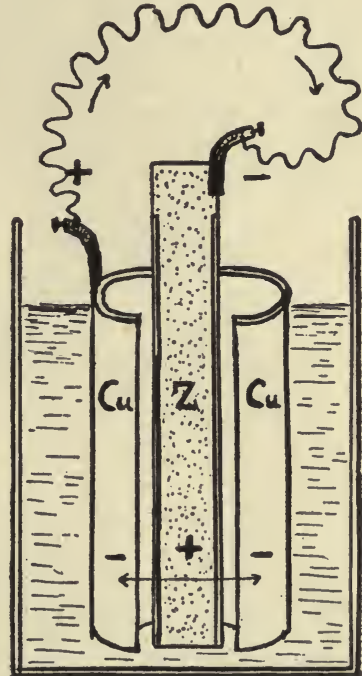

Fig. 2.-DiAgram oF Daniell Cell.

$C u$, Copper plate $(+) ; Z$, zinc plate (-). The direction of the current is indicated by the arrows. corresponds to a reservoir of electricity which remains filled as long as there is enough material present to yield chemical energy. When this material has been used up, the difference in the electric potential disappears and the current ceases.

In its passage through wires the electric current loses a certain amount of its initial energy, owing to the resistance which it must first overcome. Consequently, the strength of a current or the rate of flow 
of electricity between two different points of the conducting path is dependent not only upon the electromotive force but also upon the resistance of the conductor. A short and thick wire possesses less resistance than a long and thin wire; hence, provided that the electromotive force remains constant, the flow of electricity will be greater in the first instance. Besides this external resistance encountered by the current in its passage from the copper to the zinc, it is also opposed by an internal resistance resident in the constituents of the battery. In the latter case, the resistance is the less the larger the surface of the plates.

A unit of current is designated as an ampere, a unit of electromotive force as a volt, and a unit of resistance as an ohm. An ohm equals the resistance of a volume of mercury $1 \mathrm{~mm}$. in area and $1063 \mathrm{~mm}$. in length at $0^{\circ} \mathrm{C}$. The electromotive force of a Daniell cell is about 1 volt and that of an ordinary dry cell 1.5 volt.

The relationship existing between these different factors is expressed by Ohm's law, in accordance with which the

current strength $=\frac{\text { electrom. force }}{\text { int. res. and ext. res. }}$ or amperes $=\frac{\text { volts }}{\text { ohms }}$

Any one of these factors may be determined as follows:

$\begin{array}{ll}\text { volts } & =\text { amperes } \times \text { ohms } \\ \text { amperes } & =\text { volts } \quad \div \text { ohms } \\ \text { ohms } & =\text { volts } \quad \div \text { amperes }\end{array}$

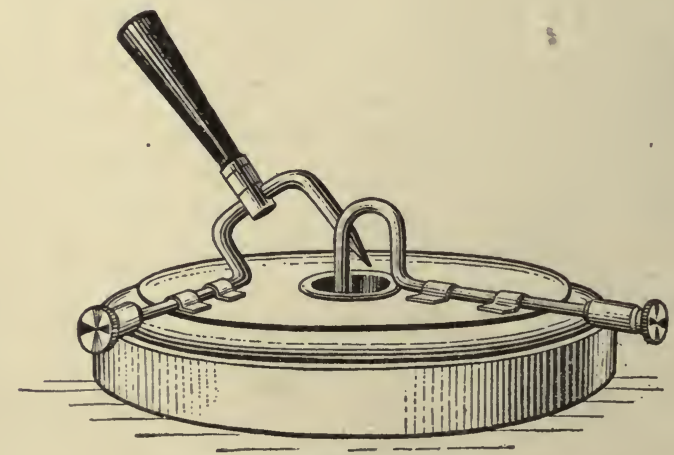

Fig. 3.-Mercury Key.

2. The Simple Key.--Living substance may be stimulated with an electric current by simply touching it with the ends of the loose wires leading out from the poles of a battery. A better way, however, is to leave the wires in firm contact with the living substance and to stimulate it by making and breaking the current by means of a key. Three kinds of keys are commonly used in the laboratory, namely, mercury, friction (DuBois-Reymond), and automatic keys.

The mercury key consists of a round wooden base weighted with iron. The center of its upper surface is depressed for the recep- 
tion of a small vulcanite cup which is partially filled with mercury. To the sides of the wooden base are attached two brass rods. The inner ends of these dip into the mercury, while their outer ends are

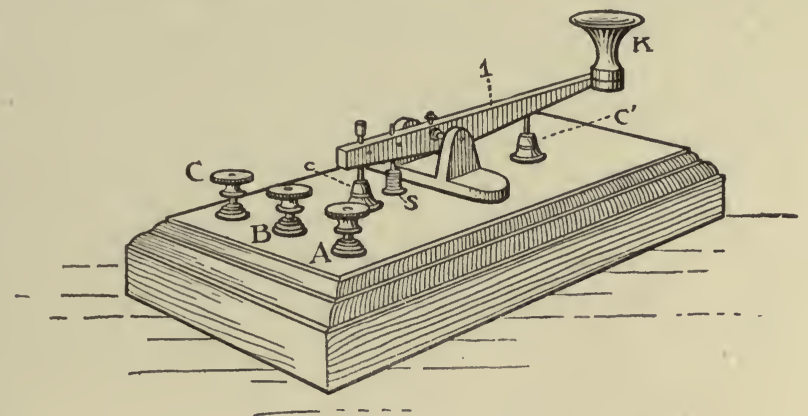

Fig. 4.-MORSE KeY.

connected by means of binding-posts with the poles of the battery. One of these rods is jointed so that its inner end may be dipped into the mercury or removed from it at will, thereby making and breaking the current. The friction key consists of a vulcanite base to which are attached two oblong bars, II and III, each of which is equipped with two binding-posts. Bars II and III are joined by a third bar, IV, which is jointed at one end and may be elevated at its other end, thereby breaking the contact between II and III. On lowering this bridge the current is made, while on raising it it is broken. The automatic key presents many forms. It consists, as a rule, of a rotating disk to which are attached a number of contacts. Since the speed of the disk and the position of these contacts may be varied, it is possible to make and break the circuit without effort and at definite intervals.

3. Stimulating Electrodes.-The electric current may be conveyed through living matter by simply applying the ends of the copper wires to its surface. The closure of the key then completes the circuit from carbon to zinc. Most commonly, however, the ends of the copper

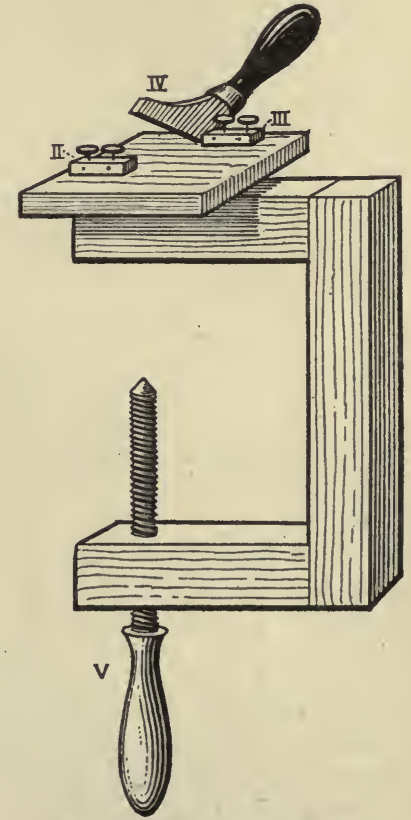

Fig. 5.-Friction Key. wires are brought close together and soldered to platinum points. They are then placed within a narrow piece of hard rubber, so that they can be conveniently handled. This form of electrode cannot be used for a long time, because the conduction of an electric current through moist 
conductors, such as are presented by animal tissue, invariably leads to a polarization of the current. The two metals of the battery, copper and zinc, are surrounded by electrolytes, the tendency of which is to pass toward the opposite pole. Thus, the positive ions, $\mathrm{Cu}$ and $\mathrm{H}$, progress toward the cathode, whereas the $\mathrm{OH}$ and $\mathrm{SO}_{4}$ pass toward the anode, which inside the cell is the zinc. Presently the copper plate becomes covered with bubbles of $\mathrm{H}$, which place a high resistance in the path of the current and eventually neutralize it. This phenomenon is known as polarization.

A similar action takes place at the points of contact between the electrodes and the tissue. It may be prevented by the use of the socalled non-polarizable electrodes. Those devised by DuBois-Reymond consist of zinc terminals immersed in a solution of zinc sulphate. A

D
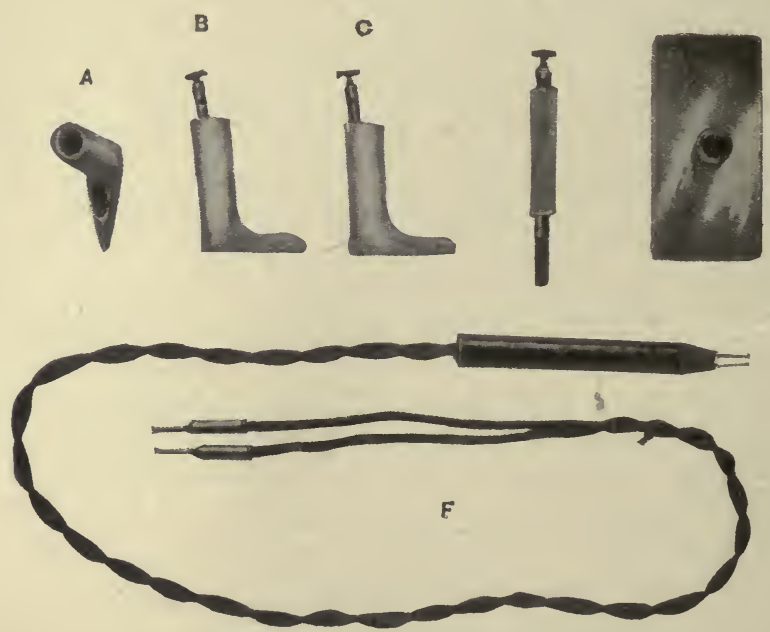

Fig. 6.-Stimulating Electrodes.

$A, B$, and $C$, Boot electrodes; $D$ and $E$, clinical electrodes; $F$, hand electrodes. (Harvard Apparatus Co.)

very simple form may be constructed by taking two pieces of glass tubing, measuring $4 \mathrm{~mm}$. in diameter and. $6 \mathrm{~mm}$. in length. One end of each tube is filled with modeling clay moistened with normal saline solution. Above the plug the tube is filled with a saturated solution of sulphate of zinc into which is placed a short rod of amalgamated zinc carrying the ends of the copper wires. The points of contact with the tissue are wrapped in cotton moistened with saline solution. These electrodes must be immersed in saline solution for some hours before they are used so as to render the clay permeable (Fig. 7).

4. The Different Types of Electric Currents.-If the two poles of a battery are connected with one another by wires and a simple key, the current begins to flow as soon as the bridge has been closed, and ceases to flow after the latter has been opened. The strength of this current 
remains the same as long as the electromotive force and the resistance do not change. A current of this kind is known as a constant or galvanic current.

If a coil of insulated wire is arranged around a ring of iron, it will be found that the passage of a constant current through the iron sets up an electric variation in the outside spool of wire. This secondary current, however, develops only on the make and break of the primary current traversing the iron. An electric energy appearing in this form is called an induced current. Moreover, since the primary current may be made and broken at long and short intervals, we recognize single make and break shocks and rapidly repeated shocks. The latter form the so-called quickly interrupted or "tetanic" current. It will be noted that the induced current is independent of the primary current, and

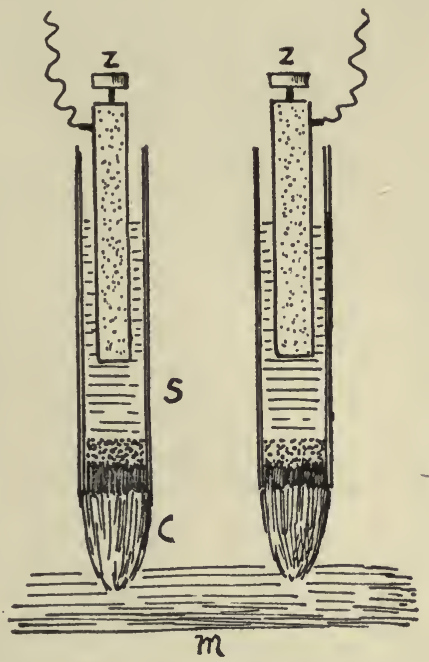

Fig. 7.-Non-polarizable Electrodes.

$M$, Muscle or nerve; $C$, cotton or camel's-hair brush; $S$, solution of zinc sulphate; $Z$, amalgamated zinc.

develops only on the make and break of the primary current. In between these two points no induction takes place, although the primary current continues to flow.

5. The Induction Coil.- The apparatus by means of which the induced current is obtained is known as an inductorium. It consists of about 130 coils of insulated copper wire of medium thickness, the terminals of which are connected with a key and the two elements of a battery. These connections constitute the primary circuit. The core of the primary coil is filled with a bundle of iron wire coated with shellac. A second spiral consisting of about 6000 coils of insulated copper wire of a thickness of $6.1 \mathrm{~mm}$. is adjusted around the primary coil in such a way that it may be moved nearer to or farther away from the primary. The two ends of the secondary wire are fastened to 
binding-posts which, in turn, may be connected with the stimulating electrodes. These connections constitute the secondary circuit of the inductorium. As has been stated above, the making and breaking of the primary current gives rise momentarily to an induced current in the secondary coil.

A rapid interruption of the primary current may be effected by closing and opening the key in quick succession with the hand. A
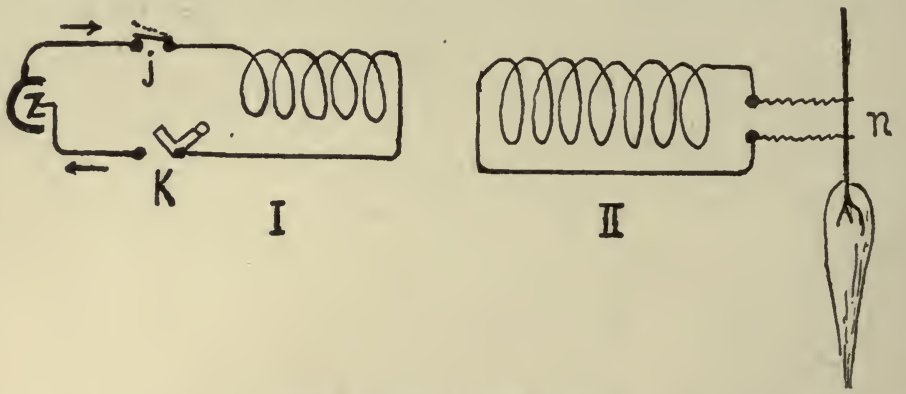

Fig. 8.-The Inductorium.

$I$, Primary circuit and coil; $I I$, secondary coil and circuit; $K$, key; $j$, automatic interrupter; $n$, nerve.

more convenient means, however, is afforded by such automatic interruptors as have been described by Neef. The latter mechanism consists of a vibrating rod and magnet, both of which are attached to the end of the inductorium. A glance at Fig. 10 will show that the current from the battery $(A)$ is led into pillar $B$ as far as the platinum contact $D$ upon the vibrator $V$. If the latter is in contact with the end of the

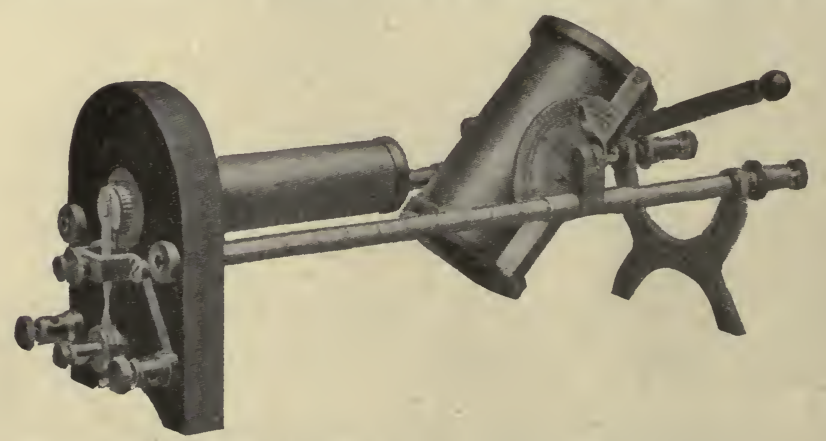

Fig. 9.-The Inductorium. (Harvard Apparatus Co.)

wire of the primary coil at $D$, the current will traverse this spiral $(P C)$ and return to pillar $F$ and the battery by way of the double spiral $E$. But as the current passes through spirals $E$ their iron cores are magnetized and attract the iron plate $H$ of the vibrator $V$, thereby breaking the contact at $D$. When the primary current is broken in this manner the spirals $(E)$ are again demagnetized. This plate $(H)$ being released, the vibrating rod moves upward and again makes contact 
at $D$. At the moment when the primary current is made and broken an induced current is momentarily developed in the secondary coil.

6. Stimulation of Muscle and Nerve-The Constant Current.-Connect in series two dry cells and a simple key and apply the ends of the wires leading away from them to the tongue. Make and break the current by closing and opening the key. When do you perceive the stimuli? Note that the make shock is the stronger of the two. Does the current stimulate while it continues to flow, $i$. e., between the make and break?

Upon the basis that a stimulus arises in consequence of any extraordinary change in the environment, it may be said that an electric cur-

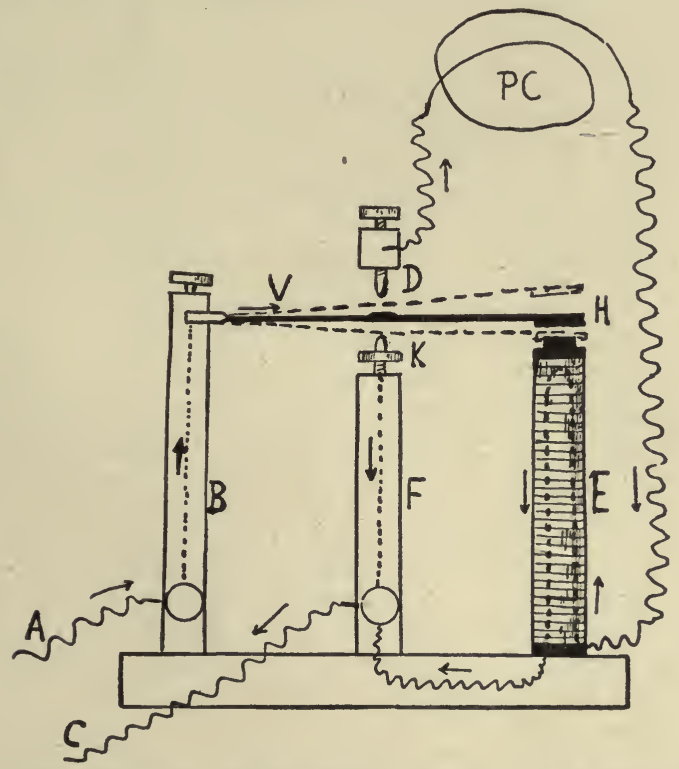

Fig. 10.-The Automatic Interrupter of the Inductorium (Neef's).

$A$, Entrance of current from battery into post $B$ and vibrator $V$ as far as $D$. In accordance with the position of the vibrating plate, the current now flows either back to the battery $C$ through post $F$ or into the primary coil $P C$ through $D$. In the latter case, the current first traverses magnet $E$ before it can reach the battery by way of post $F$.

rent stimulates only when its intensity is rapidly altered. Such alterations occur only on the make and on the break of the current, and hence, no stimulation is evoked in between these two points. Inasmuch as the full electric potential is thrown into the living substance on the make of the constant current, the latter reacts with greater amplitude at this time than later on (break), when it has been partially adapted to this stimulus.

Single Induction Shocks.-Connect in series two dry cells, a simple key, and posts 1 and 2 of the inductorium. This connection does not include Neef's automatic interruptor, and hence, the current may be made and broken at intervals of varying length. Make and break the 
primary current. When do you perceive the stimuli? Which stimulation is the stronger, the making or the breaking induction shock?

Annotation.-If the direction of the induced current is determined by means of a galvanometer, it will be found that the making shock is opposed to the primary current, while the breaking shock passes in the same direction as the primary current. Secondly, the make induction develops more slowly than the break induction, because the entering primary current must first overcome the self-induction in the primary coil before it can produce a similar effect in the secondary coil. As it passes from one turn of the primary wire to another, an induced current is momentarily set up in the secondary coil which possesses a direction opposite to the primary. Its strength is thereby diminished. On the break, this impediment is not present, and hence, the induction in the secondary coil is enabled to reach its maximal value with much greater rapidity.

\section{Quickly Repeated Induction Shocks or Tetanic Current.-Connect} in series two dry cells, a key, and posts 1 and 3 of the inductorium. This connection brings Neef's hammer into the circuit and allows us to make and break the primary current in rapid succession, thereby producing an entire series of inductions. Compare the stimulating value of the quickly interrupted or tetanic current with that of single induction shocks.

7. Changes in the Strength of the Current.-Use single induction shocks. Push the secondary coil of the inductorium over the primary. Stimulate. Gradually increase the distance between the secondary and primary coils and repeat the stimulation. What difference do you notice? Explain.

Annotation.-The strength of the induction shocks depends first of all upon the electromotive force of the primary current. Secondly, it is proportional to the separation of the secondary coil from the primary, becoming the weaker the greater the distance between them. The strength of the induction current may be indicated approximately by giving the strength of the battery and the distance between the coils in centimeters. The latter may be read off directly from the scale inscribed upon the base of the inductorium.

8. Direct and Indirect Stimulation.-Suspend the leg of a frog from the clamp in the manner described previously (p. 23). Place the sciatic nerve upon the electrodes. Moisten the preparation repeatedly with normal saline to prevent its drying. Stimulate it first with the constant and then with the interrupted current. Do the results agree with those obtained by stimulating your tongue or finger?

Hold the electrodes firmly against the body of the gastrocnemius muscle. Repeat the stimulation with the same strength of current and compare the effects of stimulating the muscle directly and indirectly through its nerve. Which is the more irritable tissue of the two, as betrayed by the threshold value of the current required to activate it?

9. Myography.-The registration of the contraction of muscle necessitates a means of holding the muscle, a writing lever, and a surface upon which the record may be made. The writing lever is fastened to the stand below the muscle clamp. The tendo achillis of the gastroc- 
nemius is then cut across and the muscle peeled off from the bones of the leg. Next the latter are cut across near the knee-joint and discarded, together with the foot. A thread is then tied around the end of the tendon and connected with the writing lever near its center of rotation. Below the tendon a slight weight is attached which is prevented from extending the muscle by an after-loading device. The electrodes are adjusted upon the upper part of the muscle (Fig. 11).

The recording surface is formed by a sheet of glazed paper which has been firmly attached to the surface of the cylinder of a kymograph and has been covered with a thin layer of soot. The smoking of the drum is accomplished most conveniently by suspending it in a smoking rack. The drum is then revolved at the rate of about once in every

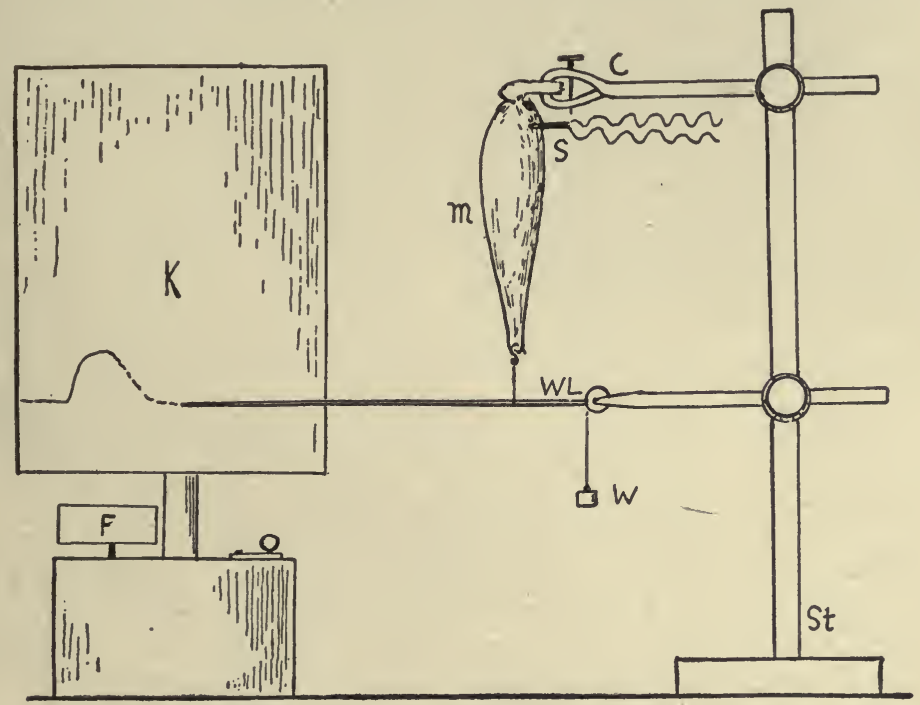

Fig. 11.-A Method Used to Register Muscular Contraction.

$S t$, Stand for holding of clamp $C$ and writing lever $W L$. The muscle $M$ is attached to the lever by means of a small hook and string. The lever carries weight $W$. The stimulation is effected through the electrodes, $S$. The speed of the kymograph $K$ may be varied by fan $F$.

second, while a broad gas flame is held directly underneath with its outer yellow zone barely touching the paper. Smoke the paper from left to right, remaining in the same place until an even brown surface has been produced. Never cease rotating the drum while the flame is directed against the paper, and do not overheat the latter, otherwise a burned gray surface will be obtained. When properly smoked, the paper should possess a velvety, chocolate-brown color (Fig. 12).

Replace the drum upon the rotating disk of the kymograph. Adjust the writing lever with the least possible friction upon the paper, and take care that it writes as a tangent upon a sphere and in a straight vertical line. The writing lever is provided with an after-loading device consisting, as a rule, of a screw-support by means of which the lever 
may be kept in the horizontal position without putting an additional weight upon the resting muscle.

Stimulate the muscle successively with the make and break shocks of a constant current, moving the drum each time by hand a distance of a few millimeters. Repeat with single induction shocks of medium strength. Allow the drum to revolve first slowly and then more rapidly, and repeat the stimulations. Obtain intermediate speeds of the drum by placing fans of different size upon the pivot of the starting gear.

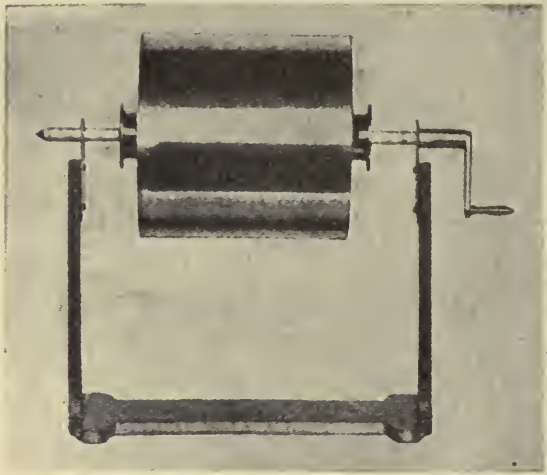

Fig. 12.-ARrangement For SMoking the PAPER. (Univ. of Penna. Lab. Outlines.) Observe the changes in the outline of the myogram as the speed is increased.

Fasten a tuning-fork, vibrating one hundred times in a second, to a separate stand, and allow its beats to be registered below the myogram.

Stimulate the muscle with a quickly interrupted current (posts 1 and 3) for a period of five seconds, and record its contraction upon a slowly revolving drum. Never permit the tracing to extend across the line where the paper has been glued together.

10. Fixation of the Records. - Write your name upon the paper and label the different tracings. Remove the drum from the stand, and hold it in your left hand with the tip of the thumb upon the edge of the paper. Insert the tip of a small forceps underneath the paper near the line where its ends have been glued together. Break the paper in a straight line. Hold the paper firmly between your hands and draw it with the blackened surface turned upward through a solution of shellac in alcohol. Suspend the paper in the frame provided for this purpose and allow it to dry. Cut out the different tracings and paste them in your note-book in an orderly manner.

11. Isotonic and Isometric Myograms.-If a muscle is made to contract against a writing lever and a slight load, a very small portion of its energy will be used up in overcoming this resistance. By far the greatest amount of its energy, however, will be set free to yield visible mechanical energy, heat, and a small fraction of electricity. If the muscle is now attached near the fulcrum of the lever, while the end near the writing point is prevented from moving by a counter-force, the shortening of its fibers will be reduced to a minimum and practically no mechanical energy will be liberated. A relatively much larger amount of the total energy will thereby be converted into heat. The former arrangement is characterized as isotonic and the latter as isometric. 




\section{LESSON III}

\section{MUSCLE AND NERVE (Continued)}

\section{IRRITABILITY, CONDUCTIVITY, AND ELASTICITY OF MUSCLE. THE POWER OF MUSCLE TISSUE IN RELATION TO THE CROSS-SECTION AND ARRANGEMENT OF ITS FIBERS}

1. Independent Irritability of Muscle.-Pith a frog and destroy its brain without losing any blood. Immediately close the opening with the pointed end of a match. Make a median incision along the posterior surface of the left thigh. Isolate the sciatic nerve carefully without injuring the femoral vessels. Tie a ligature around the thigh, exclusive of the sciatic nerve. With a large hypodermic needle inject a few drops of curare under the skin of the back. Be sure that the solution does not escape through the incision in the thigh.

After its absorption the curare circulates and is carried to all the tissues except those of the ligated leg. When a complete motor paralysis has been established in about fifteen to twenty minutes expose both sciatic nerves and place them in loose ligatures. Also expose both gastrocnemii muscles by cutting an oval window in the skin over each. Use weak induction shocks and stimulate the sciatic nerve on the side of the ligature (1) as well as on the normal side (2). In the same way stimulate both gastrocnemii muscles ( 3 and 4 ). The following results will be obtained: (1) positive, (2) negative, (3) positive, (4) positive.

Annotation.-A piece of solid curare (wurare or urare) is pulverized in a mortar and extracted with a solution of 0.7 per cent. sodium chlorid until dark in color. Its strength cannot be standardized, but must be determined by the physiologic result. Do not filter, but inject as it is.

Test the current by applying the electrodes directly to the gastrocnemius muscle. Use a strength of current just sufficient to cause a well-marked contraction. If a strong current is used, the paralysis must be more com-

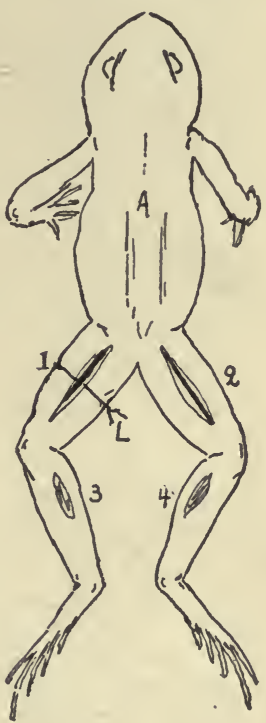

Fig. 13.-INDEPENDENT IRRITABILITY OF MUSCLE.

$A$, Dorsal lymph sac into which curare is injected; $L$, ligature upon left thigh. The stimulation of the sciatic nerve at 1 is then effective, but is ineffective at 2 . The gastrocnemii muscles, when stimulated directly at 3 and 4 , give a contraction. plete, which requires a larger amount of curare.

Curare paralyzes the motor plates in the muscle, and hence muscle (4) cannot be activated through its nerve, while muscle (3) can be. Both sciatic nerves conduct normally, as can be shown by attaching the poles of a galvanometer to them. In fact, in many instances the stimulation of the nerve on the normal side (4) produces a contraction of the gastrocnemius on the side of the ligature. The elicitation of 
this reflex proves that the impulses set up in nerve 2 are conveyed into the spinal cord and from here outward to the muscles of the non-curarized left leg. On stimulating either gastrocnemius muscle (3 and 4) a reaction is obtained. This proves that the curare establishes a block between the nerve-fibers and the substance of the muscle, $i . e$., it paralyzes the motor plates. Secondly, it may be concluded that a muscle which has been isolated in this manner from the central nervous system is still irritable and contractile. Consequently, muscle substance so isolated is capable of acting independently of the nervous tissue.

2. Irritability and Conductivity.-In order to distinguish between these two properties of living substance we usually make use of a nerve-

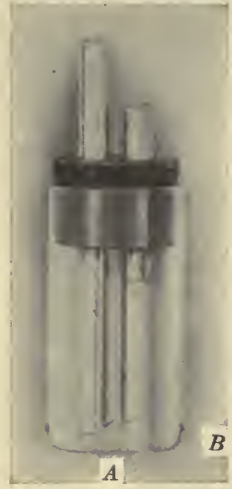

FIG. 14.-GAS Chamber for DeTERMINING THE IRRITABILITY AND CONDUCTIVITY OF Nerve. (Harvard Apparatus Co.) muscle preparation which is placed upon a plate, while its nerve is drawn through the openings of a small gas chamber. Pieces of moistened filter-paper are placed flat across these openings. Inside the chamber the nerve rests upon a pair of needle electrodes $(A)$, and outside the chamber upon a pair of ordinary platinum electrodes $(B)$. Both pair of electrodes are connected with the end-cups of a pole changer. The side cups of the latter are then united with the secondary coil of an inductorium. The inlet tube of the gas chamber is made to communicate with a generator bottle containing pieces of marble.

Stimulate successively at $A$ and $B$ with induction shocks of moderate strength. A positive result will be obtained in each case provided the nerve has not been injured. Pour a small. quantity of 20 per cent. $\mathrm{HCl}$ upon the marble. Repeat the stimulations as soon as the $\mathrm{CO}_{2}$ has begun to pass over into the chamber. It will be found that the stimulation at $A$ is now less effective than before or abolished altogether. Disconnect the generator and blow fresh air into the chamber. Stimulate again. Both excitations now give positive results.

Annotation.-The carbon dioxid destroys first the irritability and later on also the conductivity. As this gas enters the chamber containing the nerve it diminishes the irritability of the latter $(A)$, but permits its conductivity to remain practically normal $(B)$. Consequently, these properties of nerve are independent of one another.

Pour a few drops of alcohol through the inlet tube of the gas chamber, but in such a way that it does not come in contact with the nerve. Stimulate at $A$ as well as at $B$. It is to be noted that the stimulation at $B$ now remains ineffective, because the vapors of alcohol have destroyed the conductile power of the nerve-fibers, while they have diminished their irritability in a much slighter degree.

3. Extensibility of Muscle.-Use the same preparation; cut off the nerve near the muscle, and fasten the femur in the clamp. Attach the tendo achillis to the writing lever and adjust a large scale pan underneath the tendon. Release the after-loading appliance so that the 
lever may record down-strokes. Record the zero line or abscissa. Put ten weights of 10 grams each successively into the scale pan, recording each time the extension which the muscle suffers. Carefully remove the weights one by one, allowing the muscle to register its curve of detention. Does the lever return to the abscissa?

Obtain a curve of elasticity from a rubber band under similar conditions. Compare the results. Adjust the stimulating electrodes upon the upper part of the muscle. Obtain a curve of elasticity from this muscle while it is being stimulated with a tetanic current of moderate strength. What differences do you detect between this curve and that obtained under ordinary conditions? Explain.

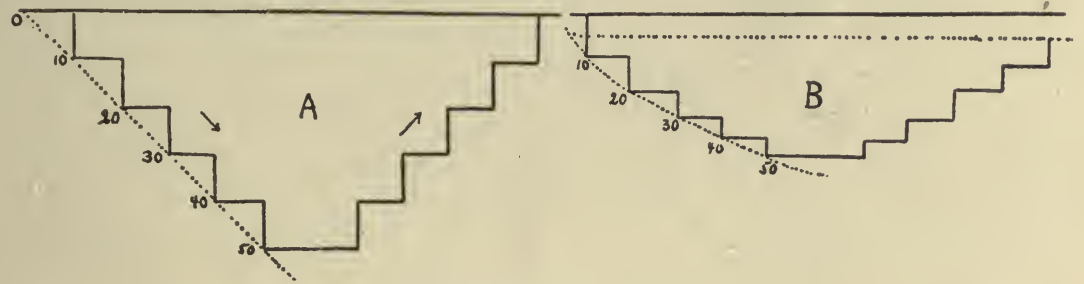

Fig. 15.-Extensibility and Elasticity.

$A$, Rubber band, and $B$, gastrocnemius muscle of frog successively loaded with $10-$ gram weights. The second curve shows a decreasing extension for equal increments, hence, the line joining the ends of the ordinates is curved.

Annotation.-A rubber band is perfectly elastic, $i$. e., it recoils until its abscissa has been reached, provided it has not been extended unduly. The same holds true of muscles in situ; in fact, they are well protected against all excessive degrees of extension. Outside the body muscles are imperfectly elastic. After their constituent fibers have been stretched they cannot resume their original shape. A fatigued muscle (tetanized) can be extended much more easily and recoils with much greater difficulty.

4. Measurement of Muscular Power.-Prepare a gastrocnemius muscle of a recently killed frog and fasten the femur in the clamp. Connect its tendon with the writing lever. Adjust the after-loading mechanism so that the lever remains horizontal. Attach weights of 100 grams each to the lever until the muscle is no longer able to lift them when stimulated with a tetanic current of moderate strength and brief duration. Determine the maximal load lifted and also the weight of the muscle. Compute the power per gram of muscle substance.

5. Comparison Between Compact and Long Muscles.-Prepare the sartorius muscle of the opposite leg of the same frog. It is situated upon the inner aspect of the thigh and extends between the ilium and the tibia. Raise its tendon at the tibia and tie a fine silk thread around it. Separate the entire muscle from the fascia connecting it with other muscles and cut its other end, leaving the ilium attached. Determine the maximal load which this muscle is capable of lifting under conditions identical with those just described. Which muscle develops 
more power, the long or the short and compact? What advantage does the latter possess over the former?

Annotation.-Short compact muscles, such as the gastrocnemius, are meant to lift heavy weights, while long, slender muscles, such as the sartorius, excel in height of contraction rather than in actual force.

6. Relation of Force to Cross-section.-Prepare the other gastrocnemius muscle of the same animal. Fasten the one already used and this one side by side in the clamp and connect their tendons jointly with the writing lever. Determine the maximal load lifted by them in the manner described previously. Quickly disconnect and arrange these muscles tandem by uniting the tendon of one to the point of attachment of the other by means of a short wire. Adjust one wire from

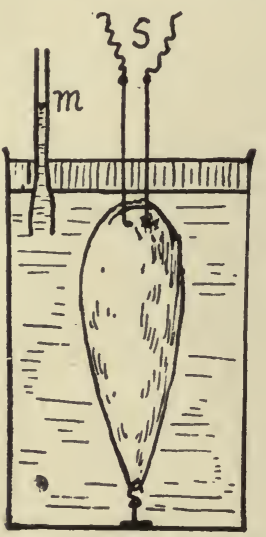

Frg. 16.-Schema to Show that Contracting Muscle Does Not Change its Volume.

$M$, Meniscus of saline solution; $S$, electrodes through which muscle in receptacle is stimulated.
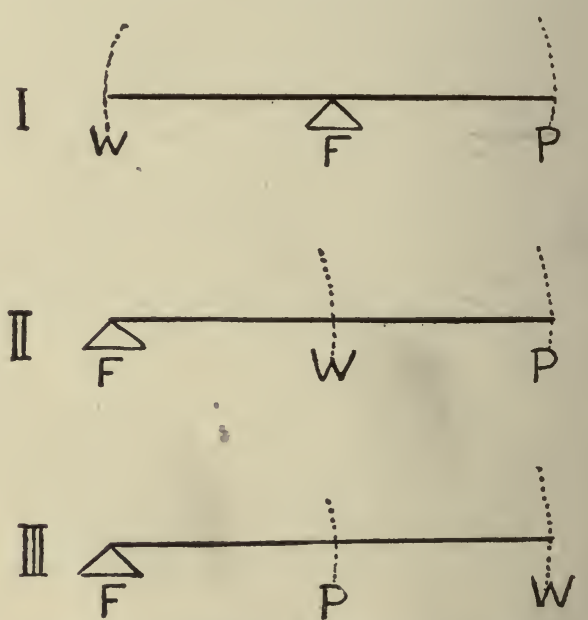

Fig. 17.-Different Systems of Levers. $F$, Fulcrum; $P$, power; $W$, weight:

the secondary coil of the inductorium to the body of the upper muscle and the other to the body of the lower muscle. Stimulate with the same strength of current used before, and determine the maximal load lifted by them when arranged in this way instead of side by side.

7. Effect of Contraction Upon the Volume of the Muscle.-Place one of these gastrocnemii muscles in the glass receptacle provided for this purpose. Be sure that its ends are firmly attached to the hooks projecting from the bottom and top of this receptacle. Fill the receptacle with boiled saline solution; close it, and adjust the capillary tube so that the "meniscus," indicating the level of the liquid, can be clearly seen. Connect the ends of the hooks with the secondary coil of an inductorium. Stimulate with single induction shocks, and note whether or no a decided change results in the position of the meniscus when the muscle contracts (Fig. 16). 
Annotation.-Since the contracting muscle does not interchange material with the medium, but merely shifts its substance, its volume is not altered. The meniscus remains stationary, provided all the air has been driven out of the saline by boiling.

8. Manner of Attachment of the Muscles to the Bones.-Determine the position of the fulcrum, weight and power in the cases of the biceps in flexing the forearm, triceps in extending the forearm, muscles upon the ventral aspect of the neck in moving the head, gastrocnemius in raising the body on tiptoe, tibialis anticus in raising the body on the heel, and the masseter in raising the jaw (Fig. 17).

9. Center of Gravity.-With chalk outline the feet of a person when standing erect. Approximately determine by means of a ruler, held vertical, the position of his center of gravity. Attach a heavy load first to the front and then to the back of his body, and note the manner and degree in which the body is shifted to support the center of gravity. What use may be made of the legs and arms in gaining additional support? Draw a diagram illustrating the progression of the center of gravity during the act of walking. 





\section{MUSCLE AND NERVE (Continued)}

\section{SINGLE CONTRACTION, SUMMATION AND FUSION OF CONTRACTIONS. TETANUS. INFLUENCE OF CHANGES IN THE STRENGTH OF THE STIMULUS AND LOAD OF THE MUSCLE}

1. Single Contraction or "Twitch."-Attach a gastrocnemius muscle to the clamp and the writing lever. Place an electromagnetic signal directly below the writing lever, so that their points come to lie close together and precisely in the same vertical line (ordinate). Fasten a scale pan underneath the writing lever, but in such a way that it cannot interfere with the movement of the lever. Connect the signal in series with the cells and posts 1 and 2 of the inductorium. Adjust the secondary coil to obtain induction shocks of medium strength. Fasten a tuning-fork to a separate stand which is placed to the left of the kymograph, so that the pointer of the fork vibrates against the rotation of

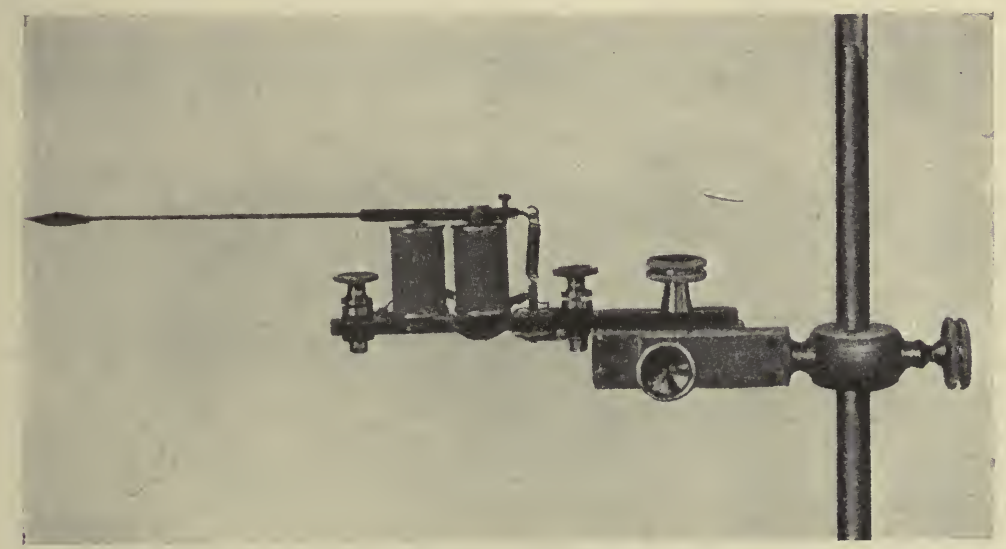

Fig. 18.-Electromagnetic Signal. (Univ. of Penna. Lab. Outlines.)

the drum. Raise the drum of the kymograph from its friction-surface by properly adjusting the screw in the top of the rod.

Let the tuning-fork register its vibrations $\left(\frac{1}{100}\right.$ sec.) directly below the record of the signal. With your left hand then spin the drum once around its axis, and with your right hand close and open the key of the primary circuit. The speed of the drum should be such that the circuit may be made and broken conveniently during a single rotation of the drum. Repeat this experiment a number of times. Place a ruler vertically against the kymograph and draw perpendiculars through the: 
(a) Moment of stimulation as indicated by the signal,

(b) Point at which the muscle just begins to shorten,

(c) Point at which the muscle shows its greatest degree of shortening, and

(d) Point at which the writing lever again reaches the abscissa.

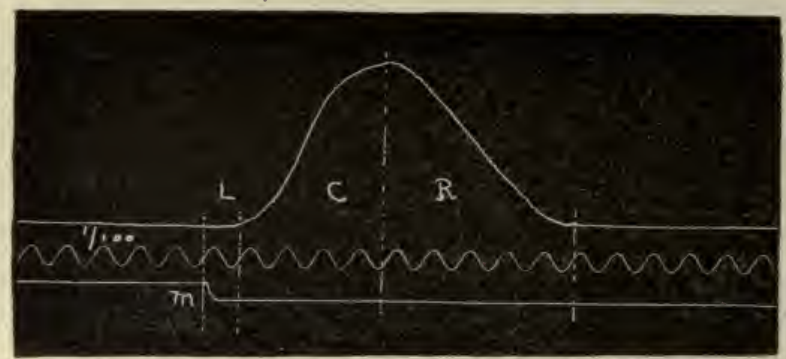

Fig. 19.-A Muscle Twitch.

$M$, Make shock recorded by magnetic signal connected with primary circuit. Time in I 10 sec.; $L$, latent period; $C$, period of contraction; $R$, period of relaxation.

Determine the duration of: (a) The latent period, (b) the period of contraction, and $(c)$ the period of relaxation. Note that the muscle does not relax properly if the stimulus is too strong, and that the length of the curve increases after a time, owing to fatigue.

2. Summation of Contractions.-Remove the tuning-fork. Use a rapid speed of drum and again stimulate the muscle with a make and

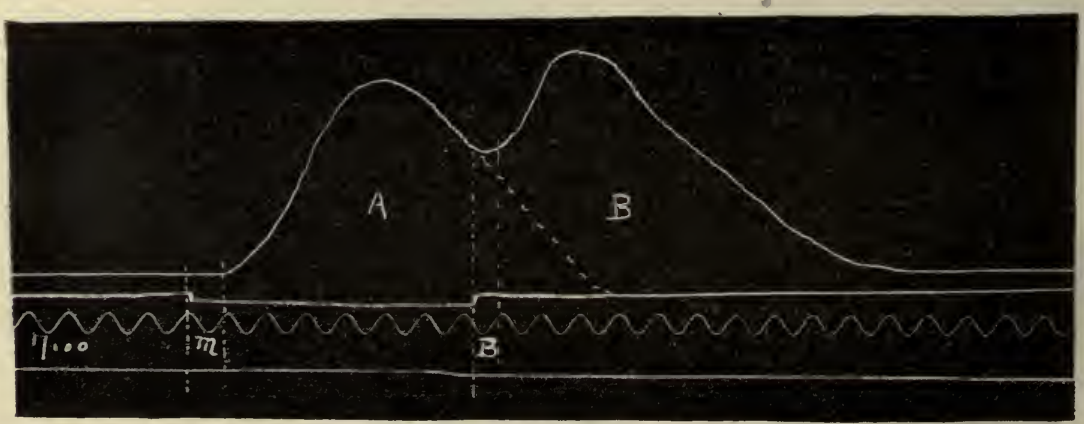

Fig. 20.-Summation of Contractions.

$M$ and $B$, Make and break shocks indicated by an electromagnetic signal. Time in $\frac{1}{100}$ sec. As the break contraction occurs during the period of relaxation of the make contraction, it is added to the first.

break shock, but in such a manner that the break stimulus strikes the muscle during its period of relaxation. A second contraction then results which is added to the first, thereby rendering it higher than the first.

3. Fusion of Contractions.-Reduce the speed of the drum. Repeat the previous experiment, gradually increasing the rate of stimulation until the contractions resulting from the separate stimuli are partially 
and completely fused. This fusion of single contractions eventually gives rise to a tetanus.

4. Compound Contraction or "Tetanus." - Connect in series two dry cells, a simple key, and posts 1 and 3 of the inductorium. Change the gears of the kymograph so as to obtain the slowest possible speed of rotation. Use a moderate strength of current and stimulate the

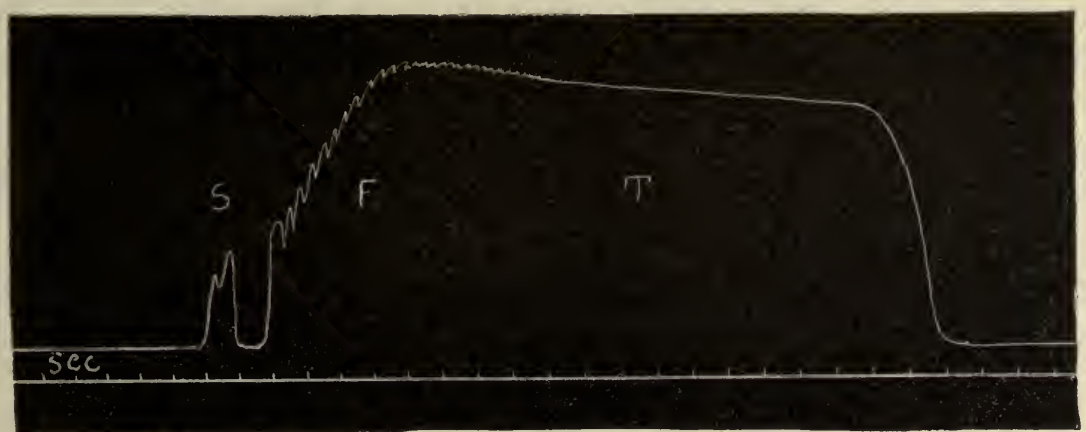

Fig. 21.-Fusion and Tetanus.

$S$, Summation; $F$, fusion; $T$, tetanus. Time in seconds. The individual make and break shocks are repeated so quickly that a continuous contraction is obtained.

muscle during a period of ten seconds. Compare this curve with the one obtained previously. Since the tetanic contraction is the result of the summation and fusion of simple twitches, it is much higher and longer than a twitch. If long continued, the height of the curve declines slowly, owing to fatigue.

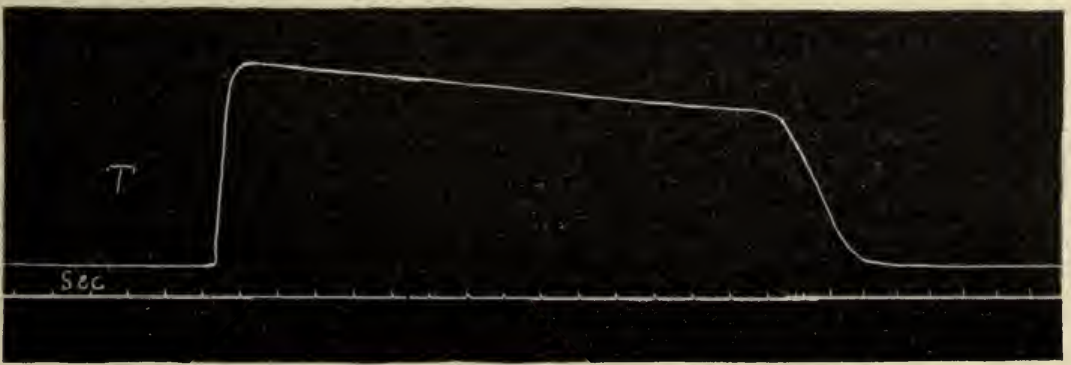

Fig. 22.-Tetanic Contraction.

Recorded by means of Neef's automatic interrupter. Time in seconds. The decline of the curve is an indication of fatigue.

5. Relation of the Strength of Stimulus to the Height of Contraction. -Prepare a gastrocnemius muscle and fasten the femur in the clamp. Connect the tendon with the writing lever, and put a 10-gram weight into the scale pan. Record the abscissa. Retain the writing lever in this line by means of the after-loading screw. Apply the electrodes firmly to the upper part of the muscle. Push the secondary coil of the inductorium over the primary. Record the make contraction, keeping 
the fingers of your right hand firmly upon the key. When the lever has again assumed the horizontal position, turn the drum with your left hand about $2 \mathrm{~mm}$. to the left, and record the break contraction. Move the secondary. coil outward for a distance of $1 \mathrm{~cm}$. Turn the drum $5 \mathrm{~mm}$. to the left. Again make and break the current as described. Repeat this experiment, constantly increasing the distance between the coils, $1 \mathrm{~cm}$. at a time, until the stimuli cease being effective. In closing and opening the key, hold the handle firmly, so that a steady contact is obtained each time. Determine the threshold values of the make and break shocks in terms of "distance of coils" and "deviation of the secondary coil." Note that the maximal reactions are obtained at about 1 or $2 \mathrm{~cm}$. distance of coils, and not when the coils are fully approximated. Beginning at this point the height of the contractions decreases gradually to zero, the make contractions disappearing sooner than the break contractions. Explain this difference upon the basis of the results of Lesson II.

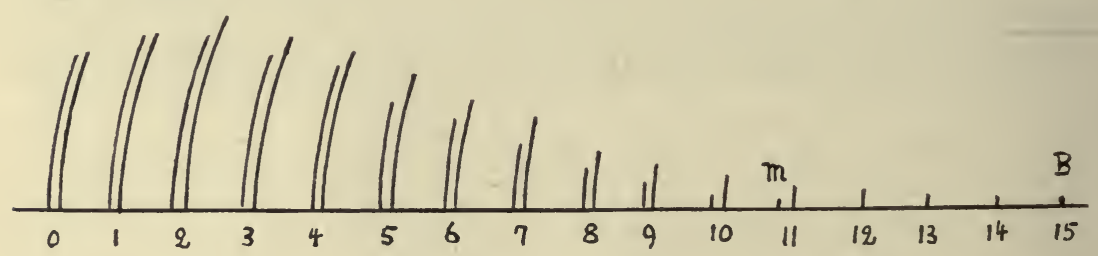

Fig. 23.-Successive Make and Break Contractions.

The strength of the current is gradually diminished by more widely separating the secondary from the primary coil. The figures indicate this separation in centimeters of distance. $M$, Threshold of make; $B$, threshold of break.

6. Summation of Subminimal Stimuli.-With induction shocks which just fail to evoke a visible contraction, stimulate the muscle at intervals of one second. Repeat the stimulation at a much faster rate. Do the subminimal stimuli eventually become supraminimal?

Annotation.-Two views may be held, namely: $(a)$ that the individual electric potentials are added to one another and finally produce a potential of sufficient magnitude to stimulate, and $(b)$ that the successive subminimal stimuli progressively increase the irritability of the muscle tissue until it eventually reacts to a stimulus which, when applied singly, does not activate it. The first view, therefore, advocates a summation of stimuli, and the second, a summation of protoplasmic excitability.

\section{Relation of the Amount of the Load to the Height of Contrac-} tion.-Prepare a fresh muscle and arrange the apparatus for stimulation with moderate break shocks. Attach a scale pan to the writing lever and record the abscissa. Keep the lever in this line by means of the afterloading device. Record a make and break contraction upon the stationary drum. In this case it is permissible to record both in the same ordinate, because the break contraction is higher than the make contraction, but do not break the circuit too soon after the make, otherwise 
you will obtain a summation. Load the muscle with a weight of 10 grams, and move the drum a distance of about $5 \mathrm{~mm}$. to the left. Repeat the stimulation. Continue to load the muscle successively with

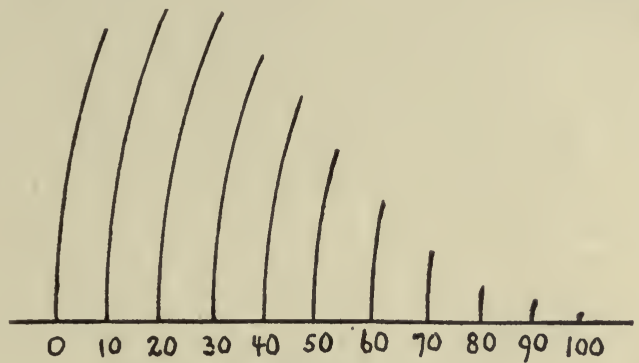

Fig. 24.-Influence of LOAD.

This muscle has been successively loaded with 10 -gram weights.

10 -gram weights. until it ceases to show an appreciable contraction. Observe the general outline of the curve obtained, and formulate a general rule regarding the height of the contraction and the load.

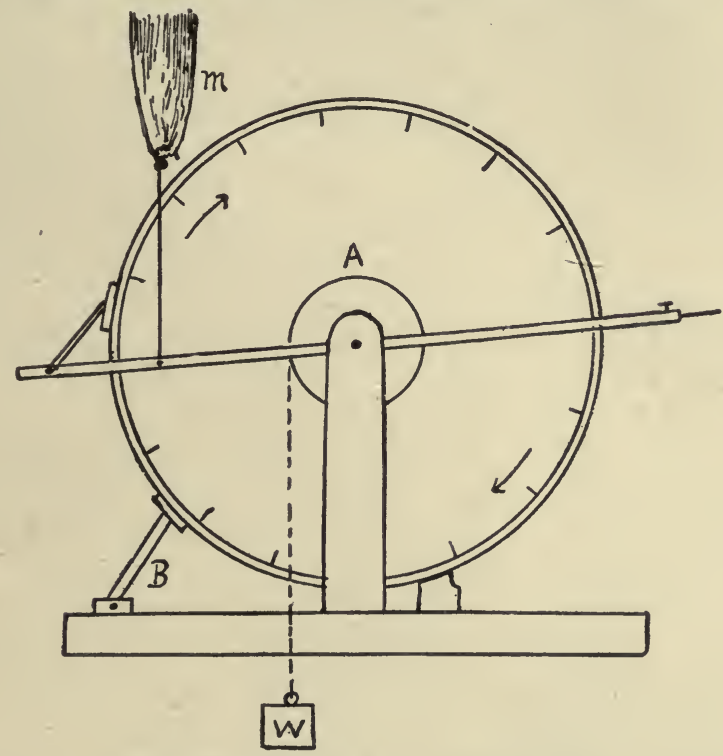

Fig. 25.-Diagram of Work-ADder.

$A$, Wheel which is turned by muscle $M$ in direction of arrows. It is held in place by brake $B$. Each contraction of muscle raises weight $W$.

8. Muscular Work.-Obtain in millimeters the values for: $L$, the total length of the writing lever, $l$, the distance from its axis to the point of attachment of the muscle, and $H$, the height of each contraction.

In accordance with the formula, $L: H:: l: h$, compute the height to which the weight has been lifted during each contraction $(h)$. The 
work $(W)$ performed by the muscle each time equals the weight $(w)$ multiplied by the height to which it has been lifted $(h)$. The work of the muscle is expressed in gram-millimeters.

9. Addition of Work.-In order to determine the amount of work accomplished by a muscle in a given period of time we make use of an instrument which is known as a work-adder (Fick). Adjust this instrument upon the edge of the table, allowing the weight (10 grams) suspended from its pulley to touch the floor. Fasten the femur of a gastrocnemius preparation in the clamp and attach its tendon to the lever of the work-adder. Apply the electrodes to the upper part of the muscle, and stimulate it at the rate of once in every second during a period of thirty seconds. The individual make and break shocks may be put in successively, $i$. e., it is not necessary to short-circuit the makes. Compute the work performed during this period by multiplying the weight by the height to which it has been lifted $(W=w h)$.

Annotation.-In short-circuiting the make, the cross-bar attached to the rods of the secondary coil is moved downward into its position of closure. The current is then made, but cannot reach the muscle because it selects the route of least resistance across the bar. Now open this bridge and break the circuit. The break shock may be short-circuited by simply closing the bridge directly after the make has been allowed to enter the muscle. 



\section{LESSON V \\ MUSCLE AND NERVE (Continued)}

\section{INFLUENCE OF TEMPERATURE, CHEMICALS, AND FATIGUE UPON THE CONTRACTION OF MUSCLE}

1. The Influence of Changes in Temperature on Contraction.-Fill the outer space of the muscle chamber with cracked ice and fasten it in the stand. Suspend a gastrocnemius muscle from the hook upon the inner surface of its cover and connect the tendo achillis by means of a long thread with a writing lever placed directly underneath the floor of

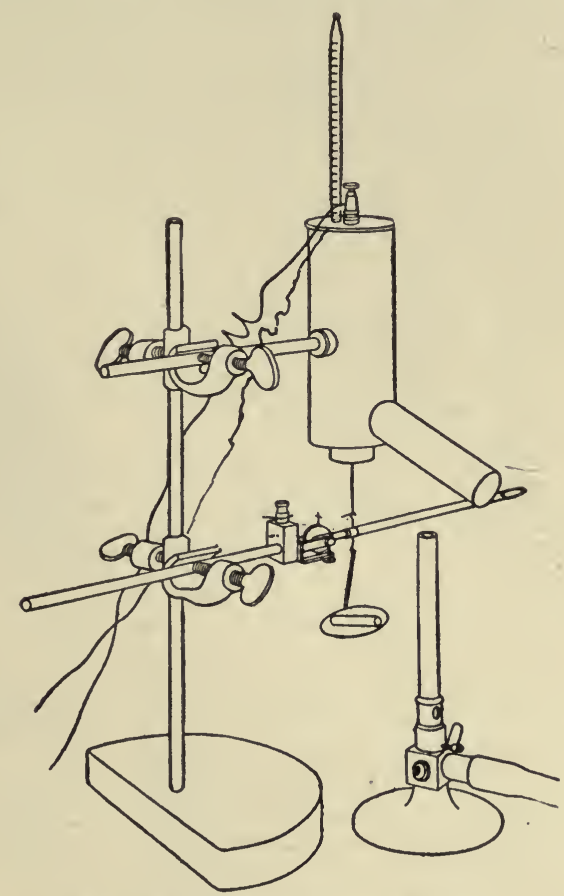

Fig. 26.-The Muscle Warmer.

An apparatus for studying the influence of temperature on muscular contraction. (Porter.)

the chamber. Fasten the ends of the wires from the secondary coil in the upper part of the muscle, and arrange the inductorium for stimulation with single induction shocks. Suspend a thermometer next to the muscle in the inner compartment, but in such a way that its bulb does not come in contact with the metal.

When the temperature has reached a point near zero, record a single break contraction upon a rapidly revolving drum. Be sure to break 
directly behind the line where the edges of the paper have been glued together. Without removing the writing lever from the drum, revolve the latter to this line. By means of an alcohol lamp gradually raise the temperature in the muscle chamber to $5^{\circ} \mathrm{C}$. Make the current. Allow the drum to revolve, and when the writing lever has passed the first contraction, open the circuit. Again rotate the drum to the aforesaid line; adjust the writing lever in the abscissa, and heat to $10^{\circ} \mathrm{C}$. Make the current and allow the drum to revolve, again breaking the circuit directly after the second contraction. Repeat this procedure at intervals of 5 degrees up to $40^{\circ} \mathrm{C}$. Contrast the sluggish contractions obtained at low temperatures with the rapid twitches obtained at higher temperatures. Is the increase in the height and decrease in the length of the individual contractions uniform?

Increase the temperature still further, and revolve the drum by hand a little at a time. At about $43^{\circ} \mathrm{C}$. the frog's gastrocnemius enters the state of heat-rigor, and finally shortens maximally. Remove the

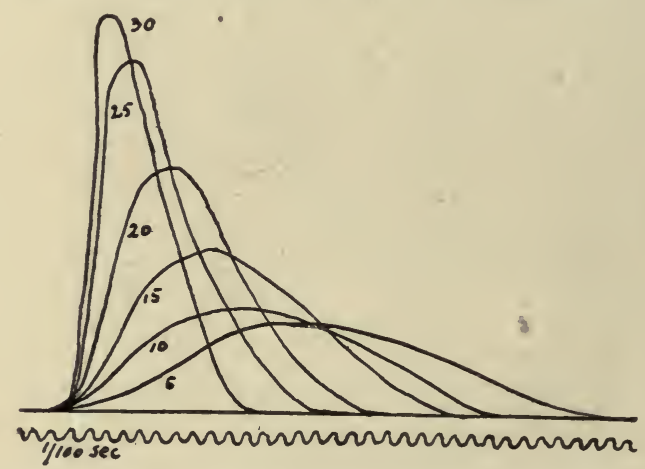

Fig. 27.-Effect of Changes in Temperature on Muscular Contraction. The temperature was raised 5 degrees each time.

muscle from the chamber and examine its texture and appearance. Stimulate it and ascertain whether it contracts.

Annotation.-By attaching an automatic key to the kymograph the current may be broken precisely at the same moment. This permits the individual contractions to become superimposed (Fig. 27).

2. Influence of Chemicals on Contraction.-Inject a few drops of a 1 per cent. solution of veratrin sulphate into the dorsal lymph-sac of a frog. Wait fifteen minutes. Prepare the gastrocnemius muscle in the usual manner, and fasten the femur in the clamp. Attach its tendon to the writing lever, and arrange the inductorium for stimulation with single induction shocks of moderate strength. Record several contractions upon a rapidly revolving drum. Study the character of the contraction of the veratrinized muscle. Wherein does it differ from the contraction of normal muscle? Also note that the veratrin 
reactions commonly alternate with perfectly normal ones. The reason for this is not known. Heat neutralizes the influence of veratrin.

Annotation.-In order to economize, the other gastrocnemius muscle of the frog used for the preceding experiment may be immersed for a few moments in a 1 per cent. solution of veratrin. If it is then adjusted in the recording apparatus, it will give characteristic veratrin contractions.

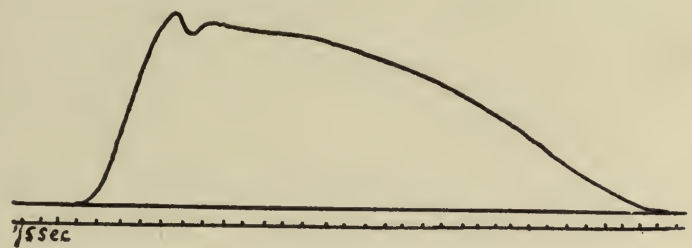

Fig. 28.-The Effect of Veratrin on Muscular Contraction.

3. The Effect of Excessive Stimulation on Contraction. Fatigue.Attach a fresh gastrocnemius muscle to the recording apparatus. Put a 10-gram weight in the scale pan. Arrange the electric apparatus for stimulation with single induction shocks of moderate strength. Make the current. Allow the drum to revolve at a rapid rate and break the current after the writing point has passed the line where the paper has been glued together. Turn the drum by hand to the end of the paper without removing the writing lever. Now stimulate the muscle at brief intervals with twenty-five make and break shocks. After the twentysixth make contraction hold the bridge of the key down. Allow the

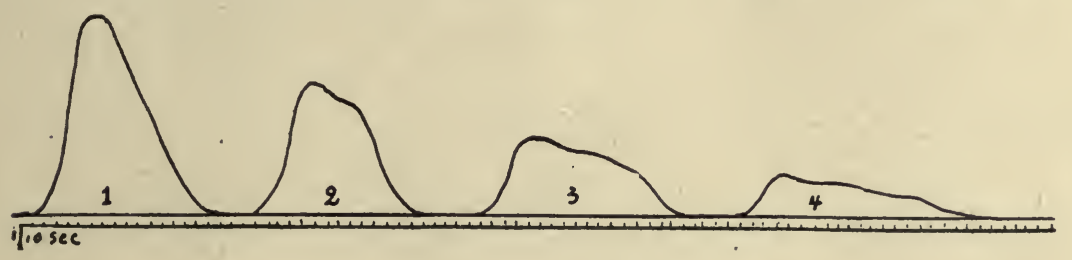

Fig. 29.-Fatigue of Muscle.

A gastrocnemius muscle of the frog stimulated successively 150 times. The 1st, 50 th, 100 th, and 150 th contractions are recorded.

drum to revolve and record the twenty-sixth break contraction directly after the first. Again stimulate the muscle twenty-five times near the line where the paper has been pasted together, and record the fiftyfirst break contraction as described. Repeat this procedure, recording the contractions at these intervals until the muscle ceases to lift the lever. Compare the different curves with one another, noting their relative heights and lengths. Wherein does fatigue betray itself? Wait five minutes and stimulate the muscle again. Does it regain its power of contraction? Compare its behavior with that of a normal muscle after excessive exercise. 
Annotation.-If this muscle possesses a high degree of irritability, increase the number of the contractions between the successive records. Since a very responsive muscle may contract as many as 750 times against a weight of 20 grams before it is fatigued, the differences between the successive contractions might not be sufficiently evident unless some such procedure is followed to hasten the onset of fatigue. Naturally, fatigue may also be brought on more quickly by increasing the weight lifted by the muscle. As has been stated above, the writing lever should not be removed from the smoked paper, because this alters the friction and interferes with the steady decline and lengthening of the successive curves.

Use the other gastrocnemius muscle of the same frog and attach it to the recording apparatus. Arrange the electric apparatus for stimulation with a quickly interrupted current (posts 1 and 3 of the inductorium). Upon a slowly revolving drum record a tetanic contraction lasting twenty seconds. Directly underneath record a second contraction of equal duration, and underneath this one, a third and fourth, until the muscle has been fatigued. 




\section{LESSON VI}

\section{MUSCLE AND NERVE (Continued)}

\section{CONTRACTION OF HUMAN MUSCLE. INFLUENCE OF BLOOD-SUPPLY. SMOOTH MUSCLE}

1. Contraction of Human Muscle.-Adjust your right forearm in the holder of the ergograph and place the middle finger in the sling supporting a weight of 2 to 3 kilos. Attach a pointer to the latter, permitting it to rest against the paper of a kymograph. Make several voluntary contractions upon a rapidly revolving drum and above the record of a tuning-fork. Estimate the amount of work accomplished each time.

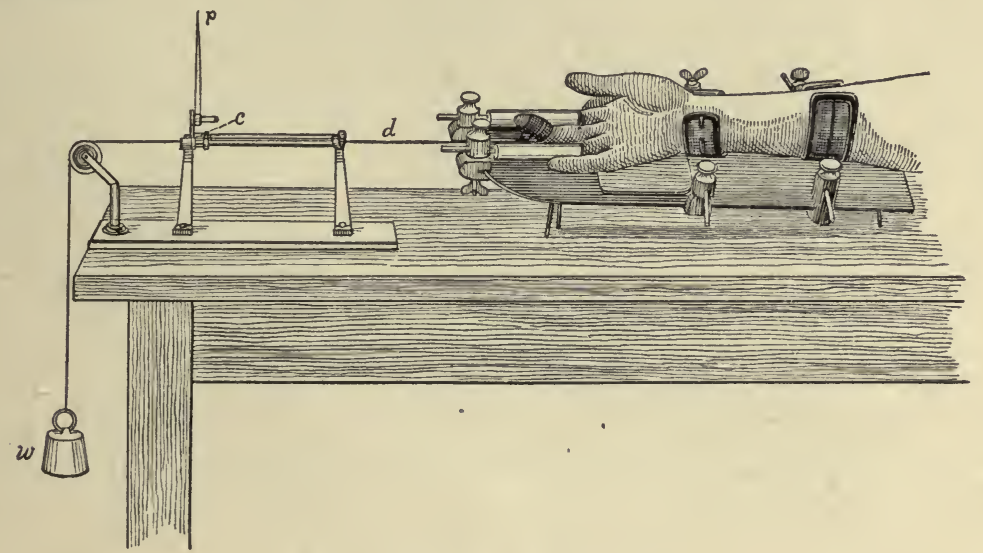

Fig. 30.-Mosso's Ergograph.

$C$ is the carriage moving to and fro on runners by means of the cord $d$, which passes from the carriage to a holder attached to the last two phalanges of the middle finger (the adjoining fingers are held in place by clamps); $p$, the writing point of the carriage, $c$, which makes the record of its movements on the kymographion; $w$, the weight to be lifted. (Howell.)

Use a very slow drum. Flex the middle finger and retain it in this position until the end of the paper has been reached (tetanic contraction). Note the oscillations and resulting fatigue.

Use a very slow drum. Flex the middle finger at a definite rate, say, once in every second, until fatigue has resulted. Allow the speed of rotation to be indicated by a chronograph beating at intervals of two seconds:

Annotation.-If Mosso's ergograph is not available, use a spring ergograph which consists of a horizontal lever attached to a metal upright. The fingers of the right hand are securely fastened in a holder, while the index-finger of the same hand is abducted against the lever. Isotonic contractions are obtained if the rod resting 
upon the index-finger and connecting the latter with the lever is moved far out toward the writing point. Isometric effects result if the vertical rod is adjusted near the metal support of the lever (Fig. 31).

2. Dynamometer.-Place a dynamometer in your right hand and contract the muscles against its spring. How great a force can you exert? Repeat with the left hand. Compare.

3. Ergographic Record of the Frog's Gastrocnemius.-Procure a metronome and insert it in the primary circuit of the inductorium. Adjust it to yield one stimulus in every second. Pith a frog and prepare a gastrocnemius muscle. Attach it to the writing lever in the usual manner. Allow the drum of the kymograph to revolve at a slow rate, and register the successive contractions of the muscle until fatigue

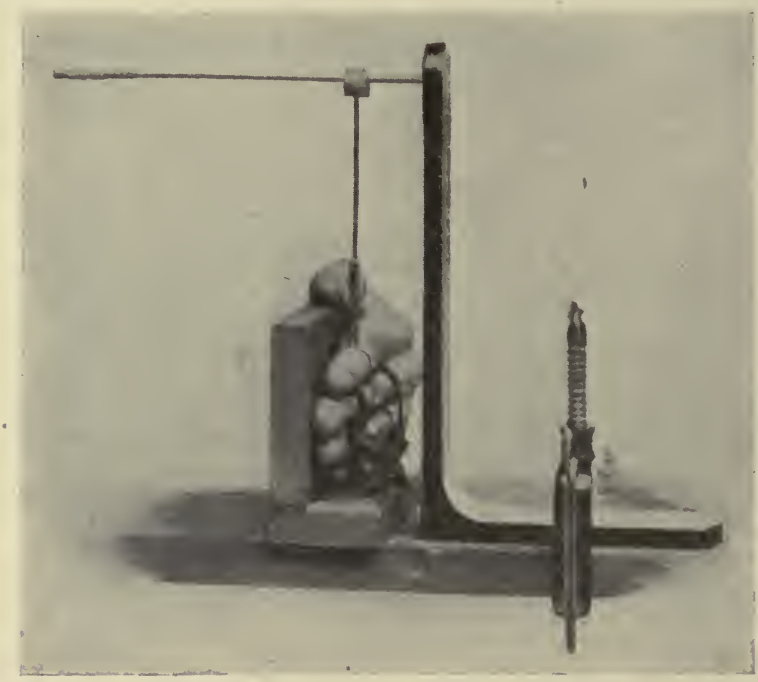

Fig. 31.-Spring Ergograph. (Harvard Apparatus Co.)

has set in. How many contractions have been obtained and during how long a period?

4. The Influence of the Blood-supply Upon Muscular Contraction.Procure a metronome and insert it in the primary circuit of the inductorium. Adjust this instrument to stimulate once in every second. Pith a frog and block the opening with a pointed piece of match so that no blood is lost. Fasten the frog upon a narrow board of cork raised to the height of the upper margin of the drum of the kymograph. Isolate the femoral blood-vessels on one side and ligate them. Make an incision through the skin of the ankle and separate the tendo achillis from the bone. Attach a silk thread to the tendon and connect it across a pulley wheel with a writing lever adjusted upon the paper of the kymograph underneath the edge of the cork board. Isolate the opposite tendo achillis in the same way, and connect it with a second writ- 
ing lever placed in the same ordinate below the first. Pull upon the strings to ascertain whether these levers interfere with one another. Attach a weight of 10 grams to each. Make a small opening in the skin over the upper part of each gastrocnemius muscle. Fasten the wires from the secondary coil of the inductorium near the knee-joint-one on each side. Then complete the circuit by uniting the gastrocnemii muscles by means of a short piece of flexible wire.

Allow the drum of the kymograph to revolve very slowly. Record the contractions of these muscles in the same ordinate until completely fatigued. Note that the bloodless muscle is much more easily fatigued. Explain.

5. Smooth Muscle.-Connect the secondary coil of the inductorium with the two outside posts of a moist chamber and attach two shorter wires of medium thickness to the corresponding inside posts.

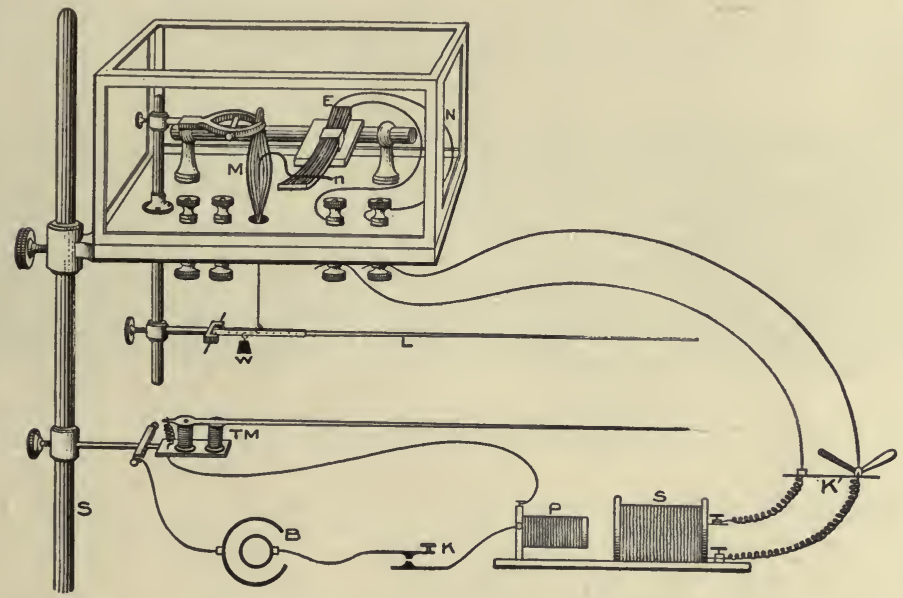

Fig. 32.-Moist Chamber.

$S$, Stand; $M$, muscle; $E$, electrodes; $L$, writing lever: $T M$, signal; $K$, simple key; $P S$, inductorium; $K^{\prime}$, short-circuiting key. (Stirling.)

Place a piece of filter-paper moistened with saline solution against the inner surface of the glass cover. If the temperature of the room is low, place a lighted alcohol lamp at some distance below the floor of the moist chamber. Arrange the electric apparatus for stimulation with single induction shocks.

Obtain a preparation of smooth muscle tissue and fasten it in the clamp at the end of the horizontal rod in the moist chamber. To its other end tie a silk thread, which is then brought through the orifice in the floor of the chamber and fastened to the writing lever directly underneath the latter. The writing lever should be adjusted in a horizontal position by moving it up or down. Do not tighten the afterloading screw, because the lever should at first be able to move upward as well as downward. Fasten the ends of the short wires in the top of the preparation. Adjust a chronograph underneath the point of the 
writing lever. Allow it to register at intervals of two seconds. Allow the drum to revolve very slowly and observe whether the muscle records spontaneous contractions.

Draw the abscissa, and adjust the writing lever properly in this line. Start at the beginning of the paper and stimulate the muscle with a single break shock. If the preparation does not react to this strength of stimulus, put in two or more shocks in quick succession-makes and breaks, if you choose. Note the unusual length of the latent period. Allow the muscle to relax fully, which, if the contraction possesses a height of about $5 \mathrm{~cm}$., may require the entire length of the paper.

Produce a summation of contractions by stimulating the muscle first by means of a make shock and again with a break shock very shortly after it has entered upon its period of relaxation. Naturally, if single stimuli do not prove effective, employ a number of them in quick succession.

Employ a brief tetanic current, being careful not to contract the muscle excessively, otherwise it might not be able to complete its period

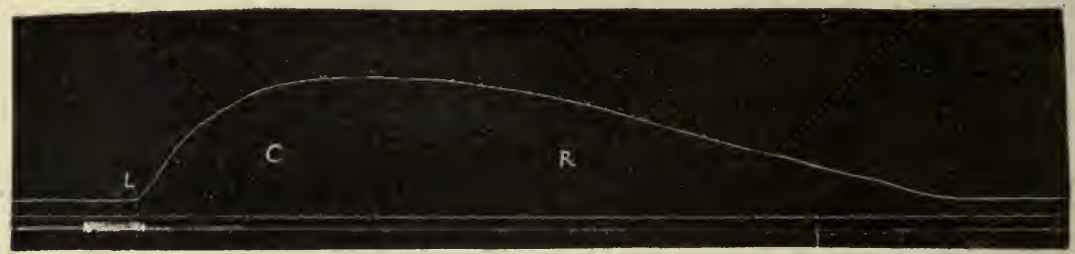

Fig. 33.-Contraction of Smooth Muscle (Cat's Bladder).

$L$. Latent period; $C$, period of contraction; $R$, period of relaxation; time in seconds.

of relaxation before the end of the paper has been reached. Does the character of the "tetanic" curve of smooth muscle differ from that of the curve obtained with single stimuli?

Annotation.-Preparations of smooth muscle may be obtained most conveniently from the stomach of the frog and the stomach, intestine, and bladder of the cat. No doubt, the bladder is best adapted for this purpose. Having opened the abdomen. in deep narcosis, the bladder is partially emptied and lifted out of the wound. A long silk thread is then tied around the urethra at a distance of 1 to 2 $\mathrm{cm}$. from the cervix of the bladder. The upper pole of the fundus is securely fastened to a pair of needle electrodes and quickly placed in the moist chamber prepared as described above. The silk thread is attached to the writing lever placed at a convenient distance below the floor of the chamber. Do not injure the organ mechanically, and moisten it from time to time with slightly warmed saline solution.

If the stomach is employed, cut a ring about $1 \mathrm{~cm}$. in width from its pyloric portion and fasten it in this position to the rod of the moist chamber. To its upper pole attach one of the wires from the secondary coil of the inductorium. To its lower pole attach the writing lever by means of a thread and also the other wire from the secondary coil. The same procedure is to be followed in the case of segments of intestine. Be sure not to arrange them horizontally, because only the circular muscle-fibers will give a marked movement of the lever. Under proper conditions of experimentation the frog's stomach will yield contractions 2 to $3 \mathrm{~cm}$. in height. 




\section{LESSON VII \\ MUSCLE AND NERVE (Continued)}

SPEED OF THE NERVE IMPULSE IN THE FROG AND MAN. CONDUCTION IN BOTH DIRECTIONS

1. Histologic Study of Nerve Tissue.-Examine nerve tissue under the low and high powers of a microscope. Employ horizontal as well as transverse sections. Identify the axis-cylinder with its fibrils, the medullary sheath, neurilemma; and nodes of Ranvier.

2. Speed of the Nerve Impulse in Cold-blooded Animals.-Place two pairs of needle electrodes upon the rod in the moist chamber, and connect them by means of wire of medium caliber with their corresponding binding-posts inside the chamber. Attach the wires from

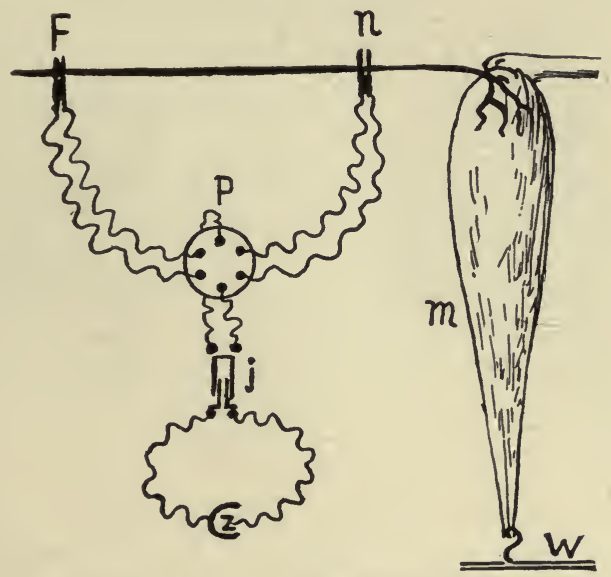

Fig. 34.-Speed of the Nerve Impulse.

$M$, Muscle and nerve connected with writing lever $W$ and two pairs of electrodes $N$ and $F$. The wires from inductorium $J$ are connected with the pole changer $P$, so that the nerve may be stimulated either near to or far away from the muscle.

the secondary coil of the inductorium to either pair of outside posts. Underneath the moist chamber adjust a writing lever and electromagnetic signal. The writing point of the latter should be adjusted in the same ordinate as the point of the recording lever. The binding-posts upon the signal are connected, on the one hand, with post 1 of the inductorium and, on the other, with the simple key. From the latter the wire is continued onward to the dry cell and subsequently to post 2 of the inductorium. Adjust the secondary coil to give induction shocks of medium strength.

Make a muscle-nerve preparation, taking care not to injure the nerve. Permit the lower segment of the spinal cord to remain attached to it. 
In the frog the cord extends as far as the ninth vertebra (at the dorsal prominence). From here the tenth vertebra or urostyle passes backward to the tip of the animal. It is best to open the abdominal cavity in the median line and to remove the viscera. Make two transverse sections so as to isolate the seventh and eighth vertebræ and roots of the sciatic nerve of one side. Trace these fibers through the pelvic aperture and down upon the dorsal aspect of the thigh. Isolate the gastrocnemius muscle and place the coiled up nerve temporarily upon it, but do not allow it to come in contact with the skin. Remove the muscles of the thigh and cut the femur. Adjust this preparation in the clamp of the moist chamber and lay the nerve across the widely separated needle electrodes. Moisten the nerve with saline solution and replace the glass cover. Test the two electrodes to see whether both are effective.

The procedure to be followed is the same as that described previously for determining the different periods of the muscle twitch. Having set

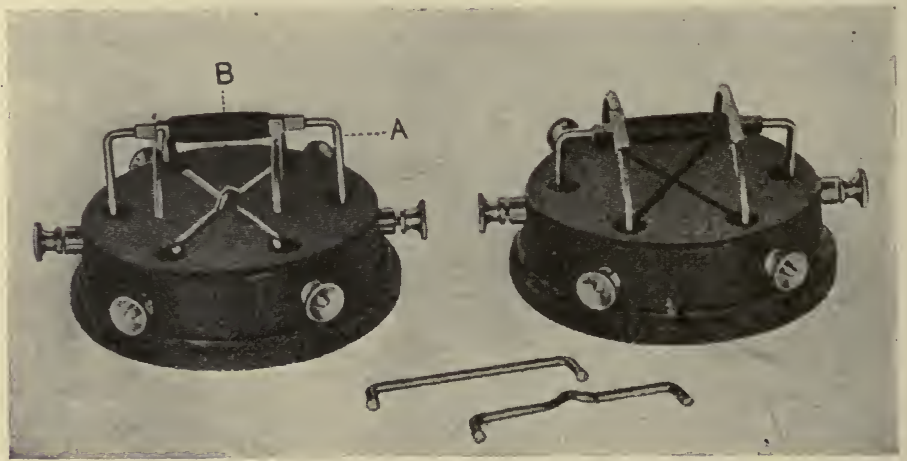

Fig. 35.-Pohl's Commutator. (Univ. of Penna. Lab. Outlines.)

the tuning-fork in vibration, the muscle is made to contract by closing the key, the contraction may occupy a single rotation of the drum, and must be repeated a number of times on far stimulation as well as on near stimulation. Obviously, the current may be diverted into either pair of electrodes by simply changing the position of the wires at the binding-posts outside the moist chamber. A pole changer may also be employed, but since this requires considerable lengths of extra wire the change may be more conveniently effected in the manner suggested above.

Draw perpendiculars to determine the length of the latent period on near and far stimulation. Measure the distance between the electrodes in millimeters. The time occupied by the nerve impulse in traversing the measured stretch of nerve, corresponds to the difference in the latent periods. Calculate the velocity of the nerve impulse.

3. Rate of the Nerve Impulse in Man.-A simple method for determining the speed of the nerve impulse in man not being available, the 
following procedure may be tried: Connect a rubber ball by means of rubber tubing with a recording tambour, and request the subject to hold the ball between his middle finger and thumb. Adjust an electromagnetic signal underneath the tambour in such a way that their writing points come to lie in the same ordinate. Place a tuning-fork fastened to a separate stand below the latter, its pointer being directed against the rotation of the drum. The binding-posts of the signal are then connected in series with a key, posts 1 and 2 of the inductorium, and a dry cell. From the secondary coil two wires are led off to a pair of clinical electrodes. The large indifferent electrode is placed upon the outer aspect of the arm of the subject, whereas the pointed one is held against the region of the median nerve at the bend of the elbow.

Allow the tuning-fork to vibrate. Spin the drum with your left hand, and close the key with your right hand. The moment of stimulation will be recorded by the signal, and the moment of contraction of the flexor muscles by the tambour. Draw ordinates and

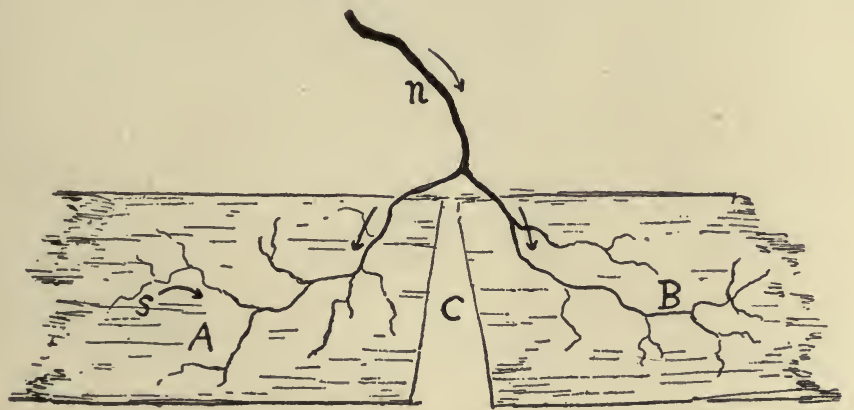

Fig. 36.-Conduction in Both Directions in Gracilis Muscle.

$A$ and $B$, Segments of gracilis muscle divided by cut $(C) ; S$, point of stimulation; $N$, motor nerve and its branches.

determine the length of the latent period. Repeat this experiment while the stimulating electrode is held over the brachial nerves above the clavicle. Again determine the length of the latent period. The difference in the latency between the two contractions corresponds to the time consumed by the impulse in its passage from the region of the clavicle to the elbow. Measure this distance, and calculate from these data the rate of transmission of the nerye impulse.

4. Nerve-fibers may be Made to Conduct in Both Directions.Pith a frog and reflect the skin from the ventral surface of the thigh. Cut transversely across the sartorius muscle near the knee and again near the ilium. Isolate the muscle along its outer border and turn its entire mass inward, so that its under surface is brought into full view. Its nerve will be seen to enter near the middle of its inner border, where it divides, sending branches toward both poles of the muscle. With the scissors cut transversely across the different muscle-fibers well into the triangle formed by the dividing nerve, so as to destroy the con- 
tinuity between the two ends of the muscle. Beginning at one end of the muscle, cut across the nerve terminals repeatedly, noting that the fibers in the other end of the muscle then contract. Do not stimulate with the electrodes, because this might invite the criticism that the contraction of the distant fibers is due to current skipping. The neighboring gracilis muscle may also be used for this experiment.

Annotation.--This motor nerve conducts ordinarily in a centrifugal direction. Since the stimulation of its terminals in one end of the divided muscle also gives rise to a muscular response in the other end of the muscle, the impulses here generated must be conveyed in an afferent direction to the nearest divisions of these efferent fibers, whence they proceed over the normally efferent fibers to the other pole of the muscle. In this way a normally efferent fiber may be made to conduct in an afferent direction. While it is probable that this nerve contains a certain number of afferent neurons, their presence cannot nullify the significance of this experiment, because in the event of their activation their impulses could only be transferred to the other end of the muscle by changing their normally afferent into an efferent type of conduction. 




\section{LESSON VIII}

\section{MUSCLE AND NERVE (Continued)}

\section{CONDUCTION IN NERVE. ACTION CURRENT OF MUSCLE AND NERVE. STIMULATION OF MOTOR POINTS}

1. Motor Nerve More Irritable Near its Center.-Pith a frog. Expose the sciatic nerve in two places, namely: $(a)$ in the pelvis by an incision between the urostyle and the ilium, and $(b)$ in the thigh (dorsal) near the gastrocnemius muscle. Insulate both segments carefully by means of narrow strips of rubber membrane. Arrange the electric apparatus for stimulation with single induction shocks and determine the least strength of make or break shocks which will give a contraction in these two places. Compare the results.

2. Influence of Temperature.-Isolate the nerve just used in its entire length, and place it upon a layer of cotton moistened with warmed saline solution $\left(40^{\circ}\right.$ to $45^{\circ} \mathrm{C}$.). Apply the electrodes to this stretch of nerve, and determine the threshold value of the make shock now required to evoke a contraction of the gastrocnemius muscle. Quickly place the nerve upon a piece of ice, and again determine the threshold of the least effective make shock. . Compare the results.

3. Galvani's Experiment.-Destroy the brain of a frog and cut away the forepart of its body. Also remove the viscera, ilium, and urostyle, but without injuring the roots of the sciatic nerves. The latter then form the only connection between the lower end of the spinal cord and the legs. Remove the skin from the latter and pass a copper hook through the spinal segment. Suspend this preparation from an ordinary iron tripod. Tilt the latter so that the legs come in contact with the iron. Vigorous contractions will result whenever the legs touch the iron. Explain, giving Galvani's as well as Volta's view.

4. Action Current of Muscle. Rheoscopic Frog.-Prepare two gastrocnemii muscles with long segments of nerve. Place both upon a plate at some distance from one another, allowing the end of nerve $A$ to rest upon the raised electrodes, and nerve $B$ of the other muscle lengthwise upon muscle $A$. Moisten this preparation with saline, but do not allow any of this solution to flow upon the plate. Arrange the induction coil for stimulation with quickly repeated shocks. Stimulate nerve $A$ briefly, noting that muscle $B$ reacts simultaneously with muscle $A$.

Annotation.-The impulses set up in nerve $A$ by the stimulation produce a contraction of muscle $A$. Since the different segments of this muscle do not react simultaneously but successively, certain segments of it must be at rest and others active. The active ones are galvanometrically negative to the resting ones, and hence a difference in electric potential is set up in this muscle which is sufficient to 
stimulate. Consequently, the response of muscle $B$ is the result of impulses which are produced in nerve $B$ by the current of action of muscle $A$.

5. Action Current of Nerve.-Cut nerve $A$ near the muscle and place it alongside nerve $B$, so that the two overlap for a distance of about $2 \mathrm{~cm}$. Stimulate nerve $A$ briefly and note that muscle $B$ contracts.

Annotation.-Nerve tissue shows a difference in potential in accordance with the progressive activation of its segments. This current of action in nerve $A$ is responsible for the excitation of nerve $B$ and the contraction of the muscle. The fact that it is not due to a direct escape of the faradic current may be proved by placing a ligature upon nerve $B$. This effectively destroys the response of the muscle.

6. Action Current of the Frog's Heart.-The fact that the beating heart generates an action current may be easily proved by exposing the heart of a rabbit during deep narcosis and placing the nerve of a muscle-

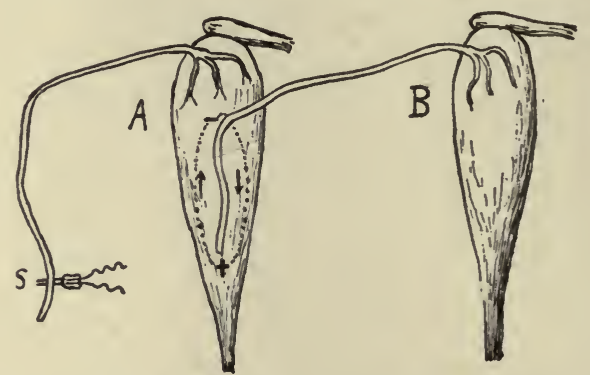

Fig. 37.-The Rheoscopic Frog Prepatation.

Muscle $A$, stimulated through its nerve at $S$, generates an action current which causes muscle $B$ to contract.

nerve preparation lengthwise upon its surface: The muscle then responds to every systole of this organ.

While the uninjured heart of a frog does not show this phenomenon very readily, it may be obtained in the following way: Excise the heart of a pithed frog and permit it to continue its activity upon a plate. Cut off its apex and apply to it the cut surface of the sciatic nerve. Place the more distant segment of this nerve upon the surface of the basal portion of the ventricle. The muscle then twitches with each beat of the heart.

7. Paradoxic Contraction.-The sciatic nerve of the frog divides near the knee into its peroneal and popliteal branches. The fibers constituting these two minor nerves are not formed by a splitting of the distal axons, but arise separately from the spinal cord and merely lie alongside each other in the nerve itself. Isolate the outer or peroneal branch and divide-it near the knee. Stimulate its central end with a brief faradic current. When a certain strength of current is employed 
the muscles supplied by the popliteal branch will be thrown into tetanus. This result is not due to the transmission of the nerve impulse in a centripetal direction in the peroneal fibers and in a centrifugal direction in the popliteal fibers, but to the excitation of the latter by the action current developed in the former.

8. Nerve Currents.-Moisten a piece of koalin in normal saline solution and draw it out in two strips about $1 \mathrm{~cm}$. in width and $6 \mathrm{~cm}$. in length. Bend each at right angles and hang them over the edge of a plate of glass at a distance of about $6 \mathrm{~mm}$. from one another. Place

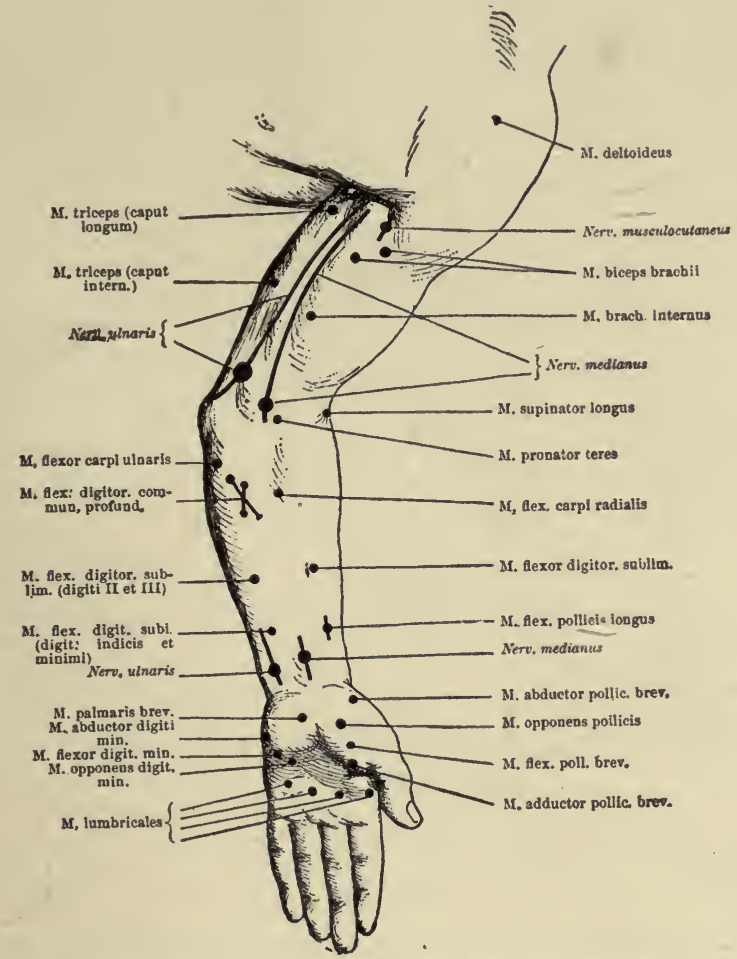

Fig. 38.-Motor Points in Upper Extremity. (Howell.)

the nerve of a nerve-muscle preparation across these strips of clay, allowing the muscle to rest upon the glass. Fill a small beaker with normal saline solution and dip the vertical ends of the clay into it. The muscle contracts every time this contact is made, because it establishes a complete circuit for the interchange of its own demarcation current.

9. Stimulation of Motor Points.-Connect the secondary coil of the inductorium by means of wires of medium caliber with two clinical electrodes (see Fig. 6, $D$ and $E$ ), one of which is flat and the other pointed. The flat, indifferent electrode is applied elsewhere to the 
integument, whereas the pointed one is used for stimulation. In order to lessen the resistance, each electrode is equipped with a layer of cotton

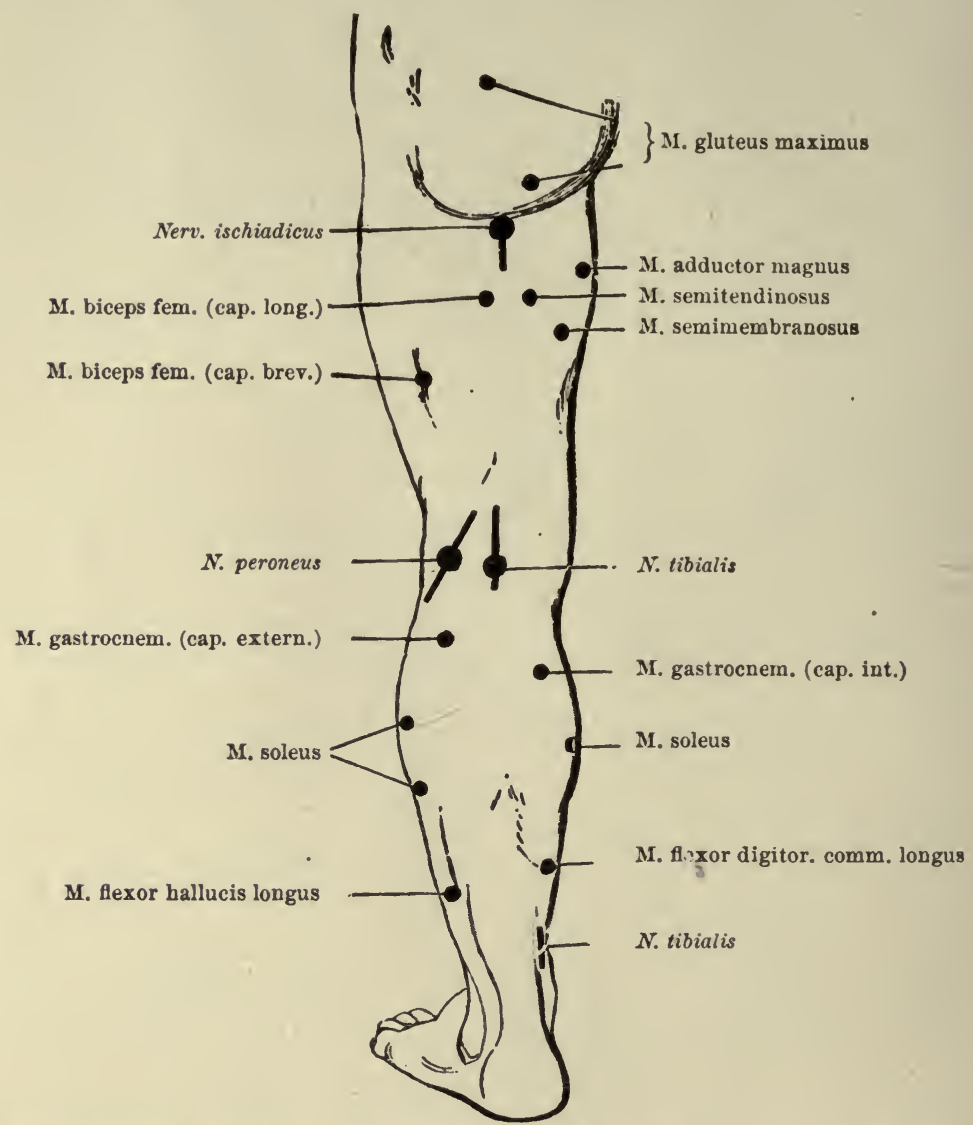

Fig. 39.-Nerves and Motor Points in Lower Extremity. (Church and Peterson.) moistened with saline solution. Localize different motor points, using Figs. 38 and 39 as guides, and stimulate them with break shocks of moderate intensity. 



\section{LESSON IX}

\section{MUSCLE AND NERVE (Concluded)}

\section{ELECTROTONUS. CATHODIC AND ANODIC EXCITATION. LAW OF UNIPOLAR STIMULATION OF HUMAN MUSCLE AND NERVE}

1. Electrotonus.-When a nerve is traversed by a constant or galvanic current it undergoes certain physicochemical changes which betray themselves in a change in its irritability at the points of entrance and exit of the current. When the key is closed the excitation producing the nerve impulse is had at the cathode (-). The anode $(+)$ is depressed at this time. On opening the key the excitation is had at the anode, while the cathode is depressed. The former condition is known as anelectrotonus, and the latter as catelectrotonus. Obviously, during the passage of the constant current, we obtain only the condition of anelectrotonus, while the catelectrotonus develops in the wake of the current, $i . e$., after it has been broken.

Anodic Depression.-Fasten two holders to the rod in the moist chamber and secure in each a non-polarizable electrode. A convenient form of the latter is the clay-boot, containing a rod of zinc. To begin with, these boots should be immersed in normal saline solution for a period of about twentyfour hours to render the clay permeable to the electric current. They are then fastened in the holders. A zinc rod having been

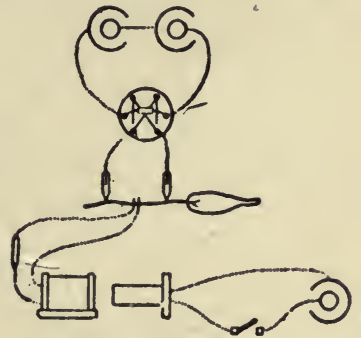

Fig. 40.-METHOD OF Testing the Electrotonic Condition of Nerve. (Porter.) placed in each, the remaining space is carefully filled by means of a dropper with a solution of zinc sulphate (see Fig. 7).

Connect in series the binding-posts upon the rods, a key, and two dry cells. Place a small tuft of cotton moistened with normal saline solution upon each boot. Make a nerve-muscle preparation, and place the nerve across the tufts of cotton, adjusting the distance between the electrodes to suit the length of the nerve. Make and break the current by closing and opening the key. With this strength of current a contraction is obtained only on the make of the current. Which electrode is connected with the carbon $(+)$ of the battery? Is the current ascending or descending in its direction?

Arrange the inductorium for stimulation with single induction shocks. Apply the hand-electrodes to any part of the nerve and accurately determine the strength of the make shock which is required to give a just perceptible contraction. Now close the key in the circuit of the constant current, and while this current is allowed to flow, stimulate the region 
of the anode of the nerve with a make shock possessing, as has just been determined, a threshold value. The anelectrotonic diminution in the excitability of the nerve will, in all probability, now render this stimulus ineffective.

If not sufficiently decisive the depression may be increased by the addition, of another dry cell or two. It eventually becomes so strong that it prevents the passage of the nerve impulse developed at the cathode. In order to show this blocking effect place the anode near the muscle, $i$. e., render the current ascending. On the make of this current the excitation is developed at the cathode. The resulting nerve impulse travels toward the muscle, but never reaches it, because it is blocked by the area of depression in the region of the anode.

This point may also be proved by placing a drop of a solution of sodium chlorid upon the nerve in the region of the anode, the anode

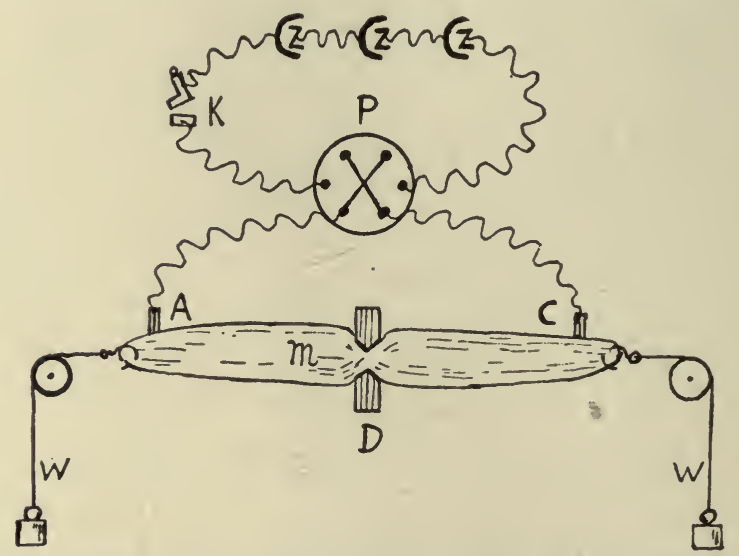

Fig. 41.-Method Used to Show Electrotonic Changes on Making and Breaking of Galvanic Current.

$K$, Key for making and breaking of current; $P$, pole changer for making either end of muscle $(M)$ anodic or cathodic; $D$, clamp applied to muscle to destroy contraction wave, but not wave of excitation; $W$, weight sattached to ends of muscle. These may be displaced by writing levers.

being in this case situated near the muscles. Presently the muscles of this leg will begin to twitch and finally become tetanically contracted. Close the key in the circuit of the constant current. The excitability of this region now having been diminished, the muscles will relax or at least show a slighter degree of tetanus. Quickly change the wires so that the cathode comes to lie near the muscle. The limb again becomes tetanic. Repeat this test.

Knowing these facts, explain the third phase of the law of Pflüger, pertaining to the effect of the constant current upon normal excised muscle and nerve.

2. Cathodic and Anodic Excitation.-When the constant current is made, the stimulation arises at the cathode, and when broken, at the anode. Suspend a sartorius muscle of a curarized frog vertically from 
a hook. Place two non-polarizable electrodes against the opposite borders of its upper portion. Make and break the current. When made, the muscle deviates toward the cathode, and when broken, toward the anode.

Slit this muscle longitudinally to near its upper end. Place a piece of rubber membrane between its two halves and adjust a non-polarizable electrode upon the surface of each. On the make, its cathodic half will contract, and on the break, its anodic half.

Isolate the opposite sartorius muscle with its attachments. Adjust a screw clamp upon its central area and tighten it sufficiently so that the wave of contraction is blocked without impairing the conduction between its two ends. Suspend this preparation. Connect each end of the muscle separately by means of a fine thread with a writing lever. Adjust a non-polarizable electrode to each end. Make and break the constant current. Note that the lever attached to the cathode moves first on the make, whereas the lever attached to the anode rises first on the break (Fig. 41).

3. Law of Unipolar Stimulation of Human Muscle and Nerve.Study the arrangement and action of the apparatus, consisting of: (a) 50 dry or moist cells connected in series, $(b)$ a pole changer, and $(c)$ two clinical electrodes, one of which is broad and the other pointed. The latter is equipped with a key, by means of which the current may be made and broken. Moisten the felt lining of these electrodes with saline solution, and apply the broad indifferent one to the shoulder. The pointed stimulating electrode hold against the skin over the ulnar nerve, near the internal condyle of the humerus. To begin with, employ 8 cells, making and breaking the current while the anode is over the nerve. Reverse the current, so that the cathode now lies upon the nerve. Repeat the stimulations. Which stimulus is effective?

Increase the strength of the stimulation by the addition of several cells (possibly 4 to 6 ). Repeat the stimulations while first the anode and then the cathode lies over the nerve. Which stimuli are effective? Add other cells, and repeat the stimulations. Tabulate the results and compare them with those forming the basis of Pflüger's law of polar stimulation. State why the latter is not applicable to normal human muscle and nerve in situ?

Annotation.-Normal human muscle and nerve give the following results with the constant current:

$\begin{array}{ccc}\text { Weak. } & \text { Medium. } & \text { Strong. } \\ \text { C C C } & \text { C C C } & \text { C C C } \\ & \text { A C C } & \text { A C C } \\ & \text { A O C } & \text { A O C } \\ & & \text { C O C }\end{array}$

This shows that the cathodic closing stimulus is the strongest of all, and the cathodic opening stimulus the weakest. Explain these results. 

4 


\section{LESSON X}

\section{THE BLOOD}

\section{THE COAGULATION OF THE BLOOD. COUNTING OF THE BLOOD- CORPUSCLES}

1. Preparation of the Animal.-Place a cat under the bell-jar, containing a small sponge moistened with ether, and carefully note its behavior during the consecutive stages of the narcosis. When fully under the influence of the ether transfer the animal to the operating table, and apply a mask to its mouth, maintaining the anesthesia throughout the following experiments.

Annotation.-Like in operations upon human beings, the depth of the narcosis is ascertained by the intensity of the corneal reflex. On touching the cornea the eyelids are closed. The quickness with which this reaction takes place serves as a guide in administering the ether. If the reflex becomes sluggish in its character, give less ether. If the reflex is abolished entirely, institute artificial respiration immediately until the respiratory movements are again executed spontaneously. Also watch the character of the respiratory movements, and note the size of the pupil, because the abolition of the reflexes is accompanied by an extreme dilatation of the pupil. The action of the heart usually ceases some time after the stoppage of respiration, and hence it is possible to resuscitate the animal if prompt measures are taken. The abdominal reflex may also be employed as a guide. On tapping upon the abdomen of the animal with the flat of the hand the abdominal muscles contract, rendering the abdominal wall tense. Obviously, if the narcosis-has been carried too far, this reaction does not take place, and the hand does not rebound.

A very efficient narcosis may also be established by means of urethane or chloral. Chloretone is administered in doses of 0.25 gram per kilo of weight. Dogs should receive $\frac{1}{8}$ to $\frac{1}{4} \mathrm{gr}$. of morphin and $\frac{1}{2} 0$ to $\frac{1}{100} \mathrm{gr}$. of atropin sulphate about thirty minutes before ether is given. When chloral is administered the animal should be kept warm, otherwise an excessive loss of heat may result. Ether narcosis is adhered to in these experiments in order to give the student as much experience as possible preparatory to his clinical work.

Tracheotomy: Insertion of Cannula.-Insert a cannula in the trachea and connect it with a glass bottle containing a sponge moistened with ether. Expose the right common carotid artery and left external jugular vein and insert a straight glass cannula in the central end of the former and the distal end of the latter blood-vessel.

Annotation.-The operation of tracheotomy is performed in the following manner: Make a median incision in the skin of the neck, beginning about $2 \mathrm{~cm}$. below the larynx. Lay the scalpel aside and cut through the underlying fascia with the scissors. With the help of two forceps separate the muscles in the median line until the trachea is brought into view. Separate the latter from the fascia surrounding it, and draw a loose cotton ligature around it. With your left hand raise the trachea sufficiently to be able to make a transverse incision between two adjoining rings of cartilage. Cut forward through two rings of cartilage. Quickly insert the short arm of the tracheal cannula through this opening and tie the ligature around 
it. To hold it firmly in place carry this ligature also around the free end of the cannula. Connect the latter by means of a piece of large rubber tubing with the ether bottle.

In inserting a cannula in the carotid artery proceed as follows: Identify the sternocleidomastoid muscle. Loosen its inner border from the neighboring tissues by means of the blunt end of forceps. Retract the muscle laterally outward. In the bottom of the wound will be found the carotid artery, internal jugular vein, and vagus nerve. The carotid artery is easily recognized as a pulsating blood-vessel of considerable size. While your assistant retracts the margins of the wound, free the artery from its sheath by means of two forceps. Place a silk ligature around it and tie it. Grasp the ligature between the thumb and middle finger of your left hand, and allow the artery to rest upon the tip of your index-finger. Apply to it a spring clip at a distance of about $2 \mathrm{~cm}$. centrally to the ligature. By means of scissors held in your right hand make a transverse incision in the artery at a distance of about $2 \mathrm{~mm}$. centrally to the ligature. Insert the beveled end of a straight glass cannula through this opening (toward the heart), and secure it by means of a silk ligature.

In inserting a cannula in the distal end of the external jugular vein the same procedure is to be followed. Make an incision along the outer margin of the sternocleidomastoid muscle. Reflect the skin outward by separating it from the fascia

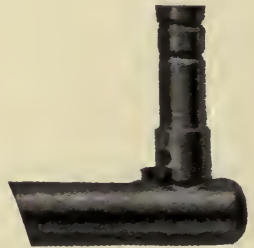

Fig. 42.-Tracheal Cannula.

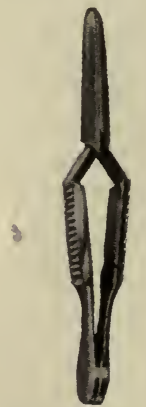

Fig. 43.-Arteiry Clamp.

(Harvard Apparatus Co.)

underneath. Cut close to the skin, because this vein lies very superficial and is easily compressed. It is recognized by its large caliber and dark color which sharply contrasts it against its investment of fatty tissue. Isolate it for a distance of 3 or $4 \mathrm{~cm}$., and ligate it with a silk thread. Apply a spring clip about $2 \mathrm{~cm}$. distally to the ligature. Incise it transversely in the manner just described and insert a cannula, securing the latter by means of a silk ligature. Since the vein collapses after the incision the opening is sometimes not easily found. The spring clip may then be opened momentarily to allow a few drops of blood to escape. Always use a cannula of about the same caliber as that of the blood-vessel into which it is inserted.

2. Arterial, Venous, and Asphyctic Blood.-Release the spring clips upon the artery and vein sufficiently to allow the cannulas to become filled with blood. Observe the difference in color. Explain. Close the tracheal tube for a short time until the animal shows forced respiratory movements. Release the clip upon the artery, and allow the blood in this cannula to be displaced by fresh blood. What is the color of the latter? Compare it with that of the venous blood previously withdrawn. Explain this difference. Clean both cannulas thoroughly by 
means of a small plug of cotton fastened to the roughened end of a short wire.

Annotation.-In operations upon animals the work should be divided in such a way that every student has his own particular task to perform. Thus, one should be assigned to give ether, another to perform the tracheotomy, and still another to cannulaize the artery. During the next laboratory period the students should be made to rotate, so that each has a different task to perform.

3. Coagulation of the Blood.-Expose the opposite external jugular vein widely. Place two ligatures about $3 \mathrm{~mm}$. apart around its central portion. Again ligate twice its distal portion. Remove the intervening segment of vein in its entirety by cutting between the two central and two peripheral ligatures. Suspend this preparation for a period of about forty minutes. Meanwhile perform the following experiments.

Annotation.--Unless the walls of the vein have been injured the blood in this segment will remain fluid for an indefinite period of time, because no agent is present therein to destroy the thrombocytes and to liberate thrombokinase.

Withdraw a small quantity of blood from the artery into a watchglass and observe the formation of the coagulum.

Allow a drop of blood to fall upon a glass slide, and observe under the microscope the clumping of the corpuscles produced by the deposition of fibrin shreds.

Draw blood into a beaker and vigorously whip it for a few minutes with a roughened piece of wood. Wash the fibrin attached to the stick, and note its appearance, texture, and elastic-properties. State why blood from which the fibrin has been removed remains fluid. Thoroughly cleanse the cannula.

Collect a few cubic centimeters of blood in a test-tube and upon a plate. Which specimen clots more rapidly?

Annotation.-Since the walls of the test-tube present a larger destructive surface to the blood, this portion will clot more speedily.

Draw a small quantity of blood into a test-tube about $1 \mathrm{~cm}$. in diameter. Note the time of its withdrawal. Hold the test-tube steady in your hand, slightly tilting it after one minute and again at intervals of one-half minute until it can be inverted without the blood flowing out. The time intervening between the withdrawal of the blood and its coagulation is known as the coagulation time.

Withdraw a sample of blood into a test-tube and place the latter in crushed ice. Since cold retards all chemical processes, it prolongs the coagulation time.

Withdraw equal quantities of blood into two test-tubes, one of which has been thoroughly anointed with vaselin. Give an explanation for the fact that the blood in the latter clots less speedily.

Draw blood into one-quarter of its volume of a 1 per cent. solution of potassium oxalate. Explain the fact that this blood remains fluid. 
Later on add a few drops of a 2 per cent. solution of calcium chlorid. Does this blood clot? Explain.

Draw blood into one-quarter of its volume of a saturated solution of magnesium sulphate. Give a reason for its remaining fluid.

Thoroughly cleanse the glass cannula, and connect. it with a short curved tube. Allow blood to enter this tube until the air has been expelled from it. Then dip the tube into a receptacle filled with mercury and allow a small quantity of blood to collect over mercury contained in a narrow vessel. Does this blood which has not come in contact with air clot?

Annotation.-Air is not an essential factor in the coagulation of the blood, and hence a perfect clot is obtained under this condition.

Draw a small quantity of blood into a test-tube. Add a few drops of distilled water. Note the changes in the color and general appearance of this blood. Give their cause. Blood so hemolyzed is known as laked blood.

To a thin layer of blood collected upon a slide add a drop of a solution of bile salts. Note under the microscope the rapid destruction of the red corpuscles.

To another layer of blood add a drop of a hypertonic solution of sodium chlorid. Study under the microscope the abnormal types of red cells. Make sketches of them in different stages of crenation.

4. Relative Amounts of Plasma and Corpuscles.-Collect 30 c.c. of blood in a test-tube. Immediately place it in the centrifuge opposite a test-tube of the same size filled with water. Centrifugalize for a period of five minutes. What is the proportion of plasma and corpuscles?

5. Enumeration of the Red Corpuscles.-Collect a drop of blood in a watch-glass. Dip into it the end of a counting pipet (Thoma-Zeiss) and aspirate gently upon the rubber tube until the pipet has been filled with blood to line 1. Quickly cleanse its end with a piece of filterpaper and dip it into Hayem's fluid. Aspirate until the bulb of the pipet has been filled to line 101. Shake the tube well. Allow a drop or two of this mixture to escape and place the next one upon the raised base of the counting chamber. Do not allow it to overflow into the space next to the base. Replace the cover, and determine under the microscope the number of red cells in 20 squares. Take the average and multiply this figure by 100 and 4000 .

Annotation.-Since each square covers an area of $\frac{1}{400} \mathrm{sq} . \mathrm{mm}$. and has a capacity of $\frac{1}{4000}$ c.mm., 1 c.mm. must contain 4000 times the average number found in these squares. Moreover, since the dilution is $1: 100$, the number of red corpuscles in 1 c.mm. of blood must correspond to the number of red cells in a square $\times 4000 \times 100$.

Hayem's fluid contains 2 grams of sodium chlorid, 10 grams of sodium sulphate, 1 gram of corrosive sublimate, and 400 grams of water.

The pipet is cleaned by drawing distilled water through it, then alcohol, and lastly ether. A current of air is passed through it from a rubber bulb until dry. 
The counting chamber is cleaned with distilled water and nothing rougher than a camel's-hair brush. Do not use alcohol or ether.

6. Enumeration of the White Corpuscles.-Proceed as before, but dilute the blood with a 1 per cent. solution of acetic acid. This agent destroys the red corpuscles. The dilution usually made is $1: 200$.
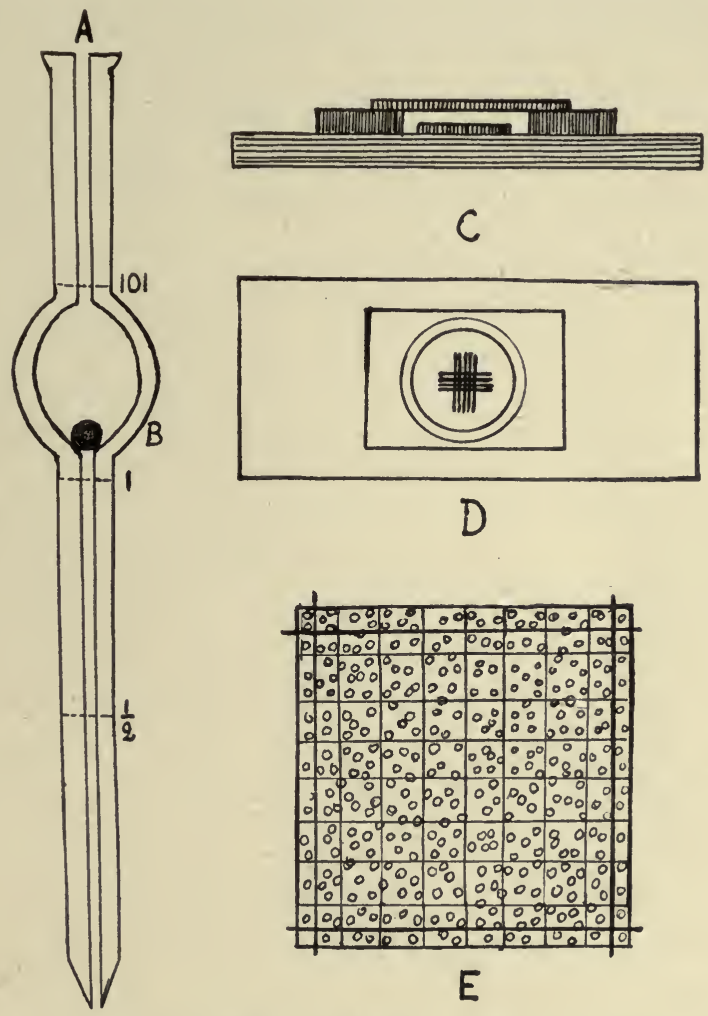

Fig. 44.-Hemocytometer. (Thoma-Zeiss.)

$A$, Pipet; $B$, glass bead; $C$, counting chamber seen from side; $D$, counting chamber seen from above; $E$, field as seen under microscope.

7. Hemorrhage.--Remove the clip from the carotid artery and allow the blood to escape into a tall beaker. Notice the differences in the force of the flow and the changes in the character of the respiratory movements, resulting in consequence of the progressive anemia and insufficient aëration of the tissues (hemorrhagic dyspnea). Kill the animal by giving an excessive amount of ether.

Place the beaker with the blood in a cool place and allow it to stand for twenty-four hours. Study the appearance of the serum and coagulum at the end of this period. 

. 



\section{THE BLOOD (Continued)}

\section{THE COUNTING OF HUMAN BLOOD-CORPUSCLES. SPECIFIC GRAVITY AND APPEARANCE OF BLOOD}

1. Microscopic Examination of Blood.-Pith a frog and open the abdomen. Incise the ventricle and allow a few drops of blood to fall into 20 c.c. of a solution of 0.7 per cent. sodium chlorid. Place a drop of this mixture upon a glass slide and examine the corpuscles under the microscope. Note their shape, size, and nucleus.

Place a droplet of blood from your finger upon a glass side and spread it out by drawing the edge of a cover-glass through it. Note the shape and size of the corpuscles. Move the cover-glass slightly so as to obtain a lateral view of some of them. Note their shape.

Insert a thin layer of porous wood under the skin of the dorsal lymph-sac of a normal frog. Allow it to remain there for twenty-four hours. Remove it and carefully wash it in a few drops of normal saline. Place a drop of the latter upon a slide and examine it for leukocytes. Study the movements of one of these, making sketches in gross outline at intervals of three minutes. If not actively moving, gently warm the slide over an alcohol lamp. Add a few granules of powdered India-ink and observe the manner in which the leukocytes envelop this foreign substance.

2. Counting of Human Red Cells.-Wash the tip of your finger with a cloth moistened in alcohol. Allow it to dry. Pierce the skin of its dorsal surface with a lancet-shaped needle and squeeze the tissues to obtain a droplet of blood as quickly as possible. Count the number of the red corpuscles in the manner described in the preceding lesson. Obtain a fresh droplet of blood and determine the number of the leukocytes.

3. Simultaneous Count of Red and White Corpuscles.-Proceed as described above when counting the red cells alone. Add a stain to the diluting fluid that will color the white cells only, for example:

\begin{tabular}{lll} 
Methyl violet, & \multicolumn{2}{c}{0.025 gram } \\
Sodium chlorid, & 1.0 " \\
Distilled water, & 100 & c.c.
\end{tabular}

Count the red cells in a group of 36 spaces. Then count the white cells in all of the 9 square millimeter spaces. Repeat with two or three different samples. Obtain the average. Multiply by 4000 and again by 100 .

4. Estimation of the Percentage of Hemoglobin.-Procure a Fleischl hemoglobinometer, glover's needle, and a small beaker. The metallic 
cup of the aforesaid instrument is divided into two compartments of equal size, and has a glass bottom and detached glass top. Added to it is a glass capillary tube held in a narrow metallic handle.

Cleanse the metallic cup thoroughly with water and dry it with a cloth if necessary. Also cleanse the capillary tube with water and hydrogen peroxid, and then again with water. Dry it by blowing a current of air through it from a rubber pouch. Fill each side of the metallic cup with distilled water-about three-fourths full.

Collect a drop of blood upon the lobule of the ear of the subject. Hold the end of the capillary tube horizontally against the drop. If the tube is clean, it will fill rapidly by capillary attraction. Remove

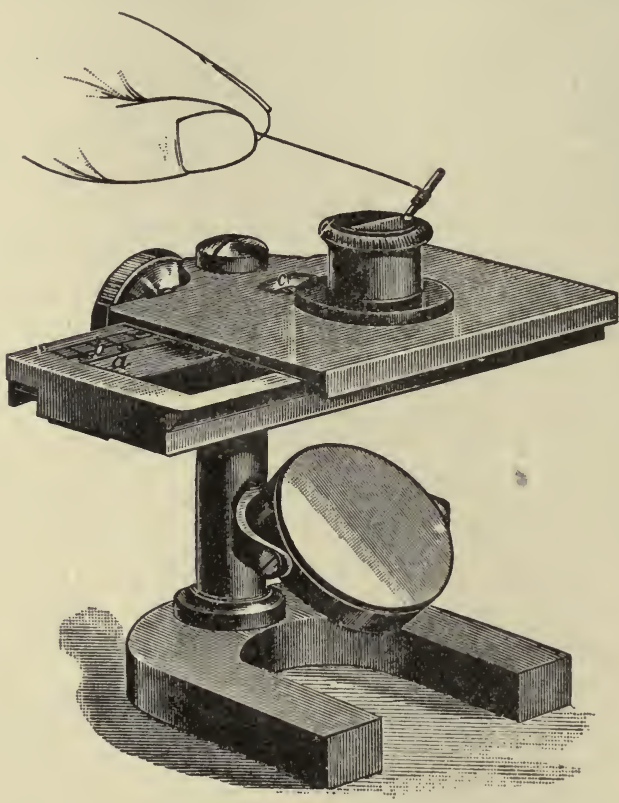

Fig. 45.-Fleischl's Hemoglobinometer. (Hall.)

excess by touching its end carefully with filter-paper. Quickly put the capillary tube in the water on one side of the metallic cup. Wave it back and forth and finally allow a few drops of distilled water from a dropper to flow through it. Fill each compartment with distilled water to the brim, stirring the mixture of blood and water until completely mixed. Close the cup with the cover-glass.

Adjust the hemoglobinometer in front of a gas-lamp in a dark room, so that the light is reflected from the mirror equally into the two compartments. Now, move the colored glass slide until the tint of the diluted blood appears to be the same as that of the colored slide. Make the reading. Repeat this procedure several times, resting your eyes repeatedly. Obtain an average reading. Thus, if the colors are 
matched, say, at division of 75 , the blood contains 75 per cent. of the normal quantity of hemoglobin.

Annotation.-Gower's hemoglobinometer, which may also be used, consists of a measuring pipet, a graduated tube, and a sealed tube containing a standard colored solution. This standard represents the color of a 1 per cent. solution of normal blood. The graduated tube is marked in 100 or more parts, each part representing 20 c.c. The capacity of the capillary tube is 20 c.c. Thus, if the blood examined is normal, it will be necessary to add water to mark 100 to make the colors correspond. If the blood is not normal, the percentage can be read off from the graduated tube above the diluted blood.

The pipet is filled in the usual way from a drop of blood collected upon the lobule of the ear of the subject. Wipe away any excess of blood after it has been filled. Then blow the blood drop by drop into the water. Shake this tube gently until a thorough mixture has been obtained. Suck a few drops of distilled water into the pipet and add this amount to the mixture. Place this tube beside the one containing the standard solution. Add distilled water drop by drop until the color
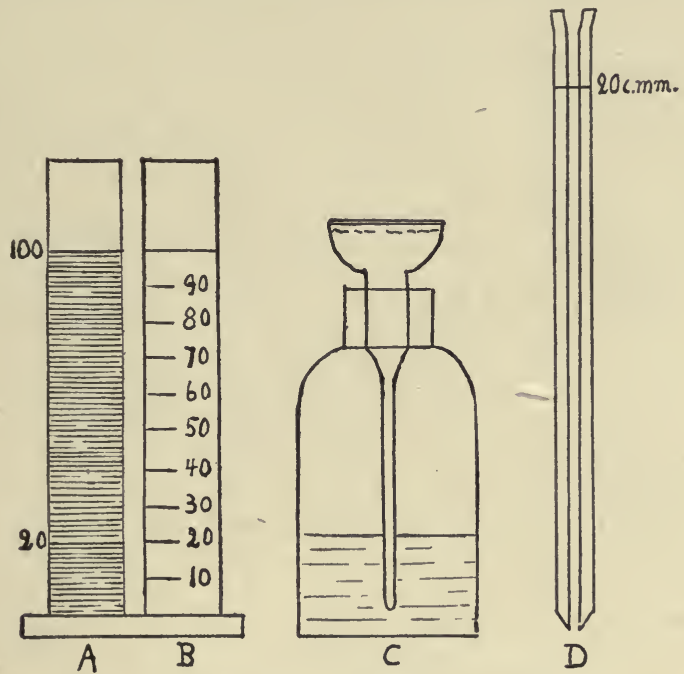

Fig. 46.-Hemoglobinometer. (Gowers.)

$A$, Tube filled with colored fluid; $B$, tube for mixing blood; $C$, receptacle for distilled water with dropper; $D$, pipet.

of the blood mixture corresponds precisely with that of the standard. Make the reading at this time, the percentage of hemoglobin being indicated upon the graduated cylinder.

5. The Specific Gravity of the Blood.-Procure a specific gravity bulb or hydrometer, a cylindric graduated glass tube about $15 \mathrm{~cm}$. in height, a pipet or pointed glass rod, a stirring rod, a glover's needle, and a mixture of benzol and chloroform. Secure a drop of blood in the usual way and allow it to fall into this mixture. If the drop of blood remains in the center of the mixture, its specific gravity equals that of the mixture. If it rises, it is lighter than the mixture, and if it gravitates downward, heavier than the mixture. In the former case, add benzol, and in the 
latter, chloroform, until the drop retains a central position. The specific gravity of the mixture is then determined by means of the hydrometer.

Annotation.-Since the specific gravity of normal blood varies with the amount of iron in the corpuscles, it must also vary with the percentage of hemoglobin. Thus, the former corresponds to the following values of hemoglobin:

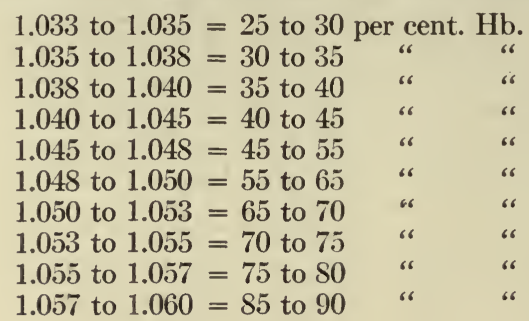





\section{LESSON XII \\ THE BLOOD (Concluded) \\ MEDICOLEGAL TESTS FOR BLOOD}

1. Spectroscopic Examination of Blood.-Place a few drops of blood in the glass cell provided for this purpose. Dilute it with water until a 0.3 per cent. solution has been obtained. Examine it with a spectroscope. Identify the $\alpha$ and $\beta$ bands. Burn a few crystals of sodium in the flame to produce a distinct Fraunhofer $D$ line.

Make a sketch showing the precise position and character of these absorption bands.

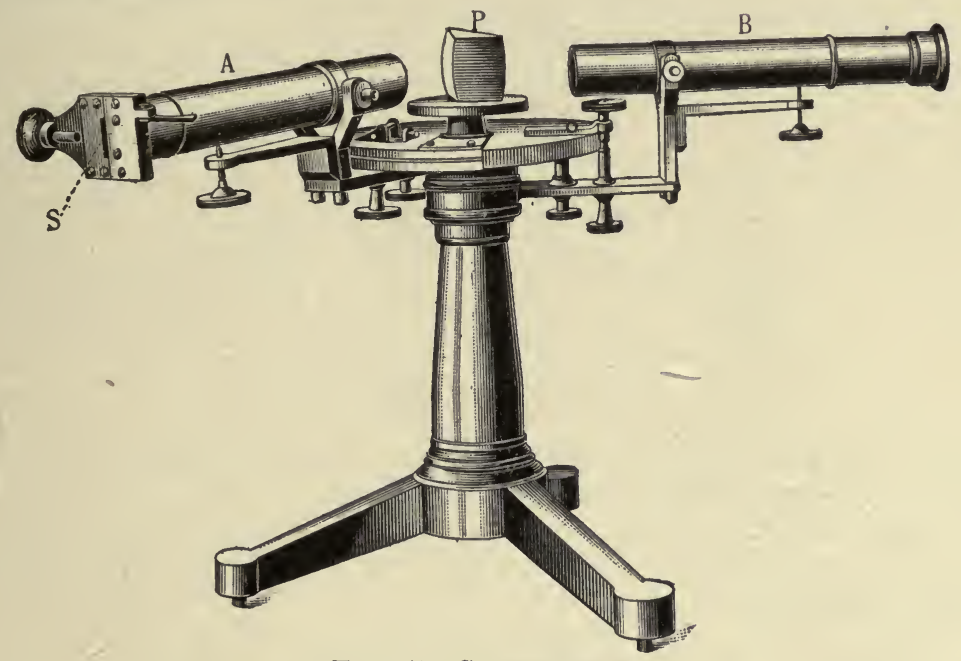

Fig. 47.-SPECTROSCOPE.

$P$, Glass prism; $A$, collimator tube, showing the slit, $S$, through which the light is admitted; $B$, telescope for observing the spectrum. (Howell.)

Reduce the oxyhemoglobin in the above solution by the addition of a few drops of Stoke's fluid. Repeat the examination. Identify the $r$ band.

Add to diluted blood a solution of caustic soda or potash, and warm. Reduce, and examine spectroscopically.

Annotation.-Greater concentrations than 0.65 per cent. produce a coalescence of the $a$ and $\beta$ bands, while very dilute solutions ( 0.01 to 0.03 per cent.) give rise to a single band, near the $D$ line. Employ solutions of 0.1 to 0.6 per cent. and use a cell the inner width of which measures $1 \mathrm{~cm}$.

Stoke's reducing fluid consists of a solution of ferrous sulphate, to which a little tartaric acid has been added. When used, add ammonia till its reaction becomes alkaline. Its color then changes from yellow to dark yellow. 
2. Examination of Suspected Blood.-Dissolve a small quantity of the suspected blood in normal saline solution. Search for corpuscles and apply the hemin test. Dissolve a small portion of the same material in water or in a dilute solution of caustic soda or potash and make a spectroscopic examination as described in the preceding paragraphs.

Annotation.-The student should at this time be shown the lytic and precipitin reactions of blood-sera. Since it often takes several weeks to sensitize an animal, these tests for blood cannot be performed by students individually.

3. Hemin Crystals.-Place a drop of blood upon a glass slide and dry it slowly in the gas flame. Add a few crystals of sodium chlorid and a drop of glacial acetic acid. Cover and gently heat until bubbles of gas are given off. Hemin crystals appear as minute, dark brown,

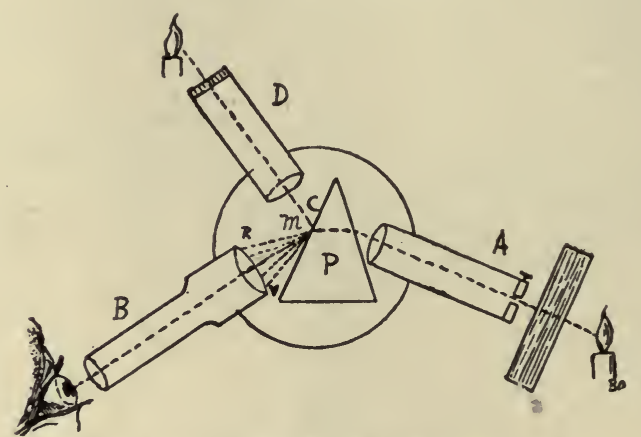

Fig. 48.-Diagram of Spectroscope.

rhombic crystals which cannot well be confounded with the irregular colorless crystals frequently seen in preparations of this kind.

4. Blood Crystals. - Mix a drop of rat's blood on a slide with a drop of water. After about five to ten minutes crystals of oxyhemoglobin will be seen to form.

5. Chemical Tests for Blood.-Add a small quantity of blood to tincture of guaiacum. Add a little hydrogen peroxid to this mixture. The blue color ensuing is due to the iron-containing radical in hemoglobin. Repeat this test with blood which has been boiled. The same reaction results.

Dilute a little blood until practically colorless. Add to it a few drops of benzidin dissolved in glacial acetic acid and a few drops of hydrogen peroxid. A blue color develops. Repeat this test with blood which has been boiled. The reaction is now less intense. 


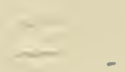

工 



\section{LESSON XIII}

\section{THE HEART}

REGISTRATION OF THE HEART-BEAT. REFRACTORY PERIOD. EXTRASYSTOLE. EXCISED HEART. ACTION OF STRIPS OF VENTRICULAR TISSUE

1. Normal Heart-beat.-Apply a ligature to the neck of an etherized turtle directly behind the occiput, and destroy the brain by pithing. Saw through the lateral aspect of the ventral shield or plastron, and remove the latter by cutting through the soft parts connecting it with the internal structures. Keep close to the bone so as to avoid the large blood-vessels. Identify the different superficial organs and note the texture, extent, and mode of attachment of the pericardial sac. Incise the latter by a longitudinal cut and separate the apex of the heart by dividing the strong band of connective tissue which unites it with the frenum. This band should be preserved to serve later on as an attachment for the hook of the writing lever.

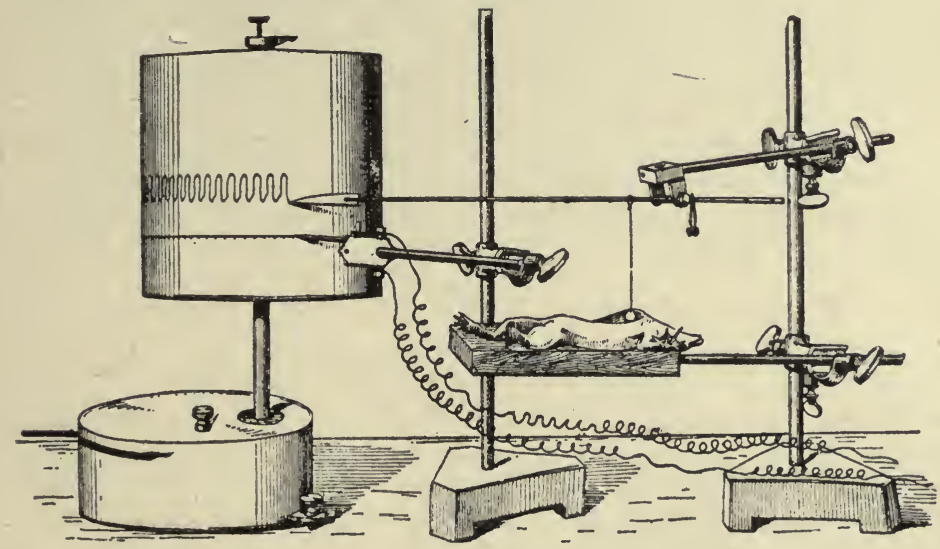

Fig. 49.-Arrangement for Registering the Contractions of the Frog's Heart. (Univ. of Missouri Lab. Outlines.)

Identify the venæ cavæ, sinus venosus, the right and left auricle, and the ventricle with its conus and main arterial trunks (aortæ). Note the color and shape of the ventricle on systole and diastole, and study the sequence of contraction of the different segments of the heart, viz., sinus, auricles, ventricle.

Insert a small hook in the apical band and connect it with a writing lever. Counterpoise the latter sufficiently to place the ventricle under a slight tension. Be sure that the plate upon which the turtle is resting is placed vertically under the writing lever, so that the heart is drawn 
upward. Allow the ventricle to register its cycles upon the smoked paper of a slowly revolving kymograph above the record of a chronograph beating once in every two seconds. What is the rate of the heart per minute? Register the auricular beats in the same manner after having previously inserted the hook in the wall of one of the auricles. Note that the rhythm is the same in the two records, and that the force of the contractions of the ventricle is much greater than that of the auricles.

Annotation.- Very good records may also be obtained from the heart of a frog. The animal is pithed in the usual way and fastened to a board by means of wire clips. A median incision is then made through the skin over the sternum, and the episternum raised with a pair of forceps. The edges of the sternum are cut through and the bone removed in its entirety. The pericardium is then lifted up and divided, thus exposing the beating heart. The sinus venosus of this heart is formed by the union of the large inferior vena cava and the two smaller superior venæ cavæ. It is continuous with the right auricle and ventricle. The latter continues as the bulbus aortæ, which gives origin to the two aortæ. From the latter arise the pulmonary arteries which supply the lungs. From here the blood is returned to the left auricle, whence it is again forced into the ventricle.

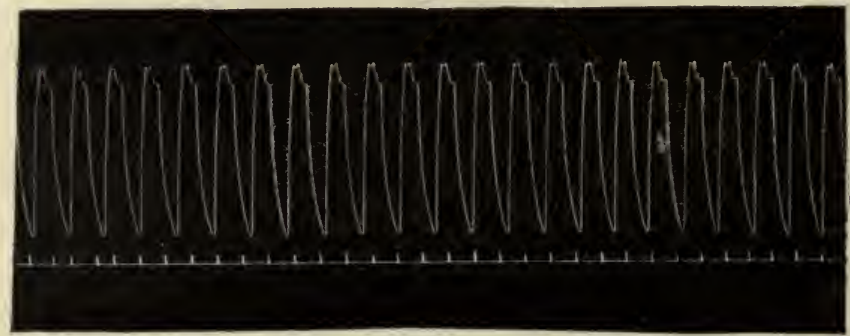

Fig. 50.-Record of the Contractions of the Frog's Heart. The time is registered in seconds.

The registration may be effected in two ways, namely, by:

(a) The Supporting Method.-A vertical rod of straw is fastened by means of a thin wire to the long arm of an ordinary writing lever. The joint between them should be very loose. The other end of this rod is made cup shaped by means of a piece of wax molded to fit the surface of the ventricle. When the latter contracts the lever is raised. Consequently, the systolic period is indicated by the up-stroke and the diastolic period by the down-stroke of each wave.

(b) The Suspension Miethod.-A fine thread is attached to the end of the short arm of an ordinary writing lever. It is connected by means of a small hook with the apex of the heart. The long arm of the writing lever is weighted sufficiently to place a slight degree of tension upon the heart. In this case the up-strokes of the record correspond to the systoles of the organ. If a spring lever is employed, the tendency of which is to move upward, the string must be fastened to its long arm. The contraction of the heart then pulls the lever downward, whereas the steel spring attached to the lever pulls it upward as soon as the cardiac musculature becomes passive during diastole.

2. Effect of Temperature.-Allow the ventricle to register its cycles upon the paper of a slowly revolving kymograph. By means of a pipet allow a few drops of iced saline solution $\left(5^{\circ}\right.$ to $10^{\circ} \mathrm{C}$.) to drop upon the 
heart. Note the reduction in the rate and slowing of each individual beat. After the heart has again resumed its normal rate and amplitude of contraction bathe it in the same manner with warmed saline solution $\left(20^{\circ}\right.$ to $25^{\circ}$. C. $)$. Note the increase in its rate, due to a greater rapidity of the individual contractions.

3. Refractory Period. Extrasystole.-Leave the heart in position, but place its ventricular portion in the cup of a heart-holder. Adjust the writing lever upon its surface, and connect the binding-posts with

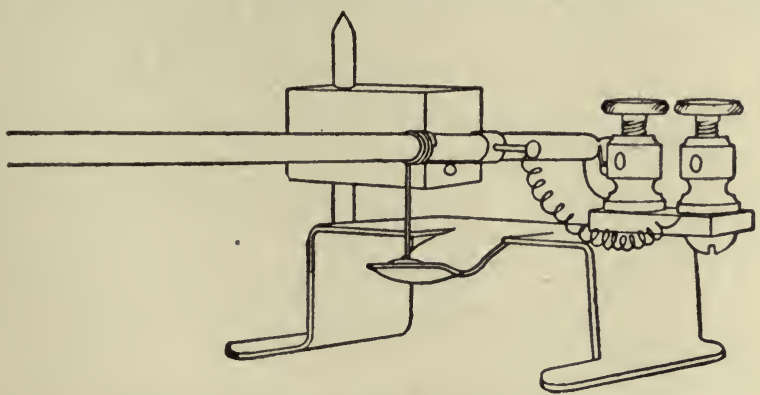

Fig. 51.-The Heart-holder. (Porter.)

the secondary coil of an inductorium. Arrange the electric apparatus for stimulation with single shocks, and insert a signal in the primary circuit. Place the writing point of the latter in the same ordinate with that of the heart lever. Allow the ventricle to register its cycles upon a drum revolving at a moderate speed. Stimulate at intervals first during the systolic and then during the diastolic period of the heart. Note that the former stimuli remain without effect, whereas the latter produce an extra contraction (extrasystole). While the musculature is in contraction it remains impervious to stimuli (refractory period).

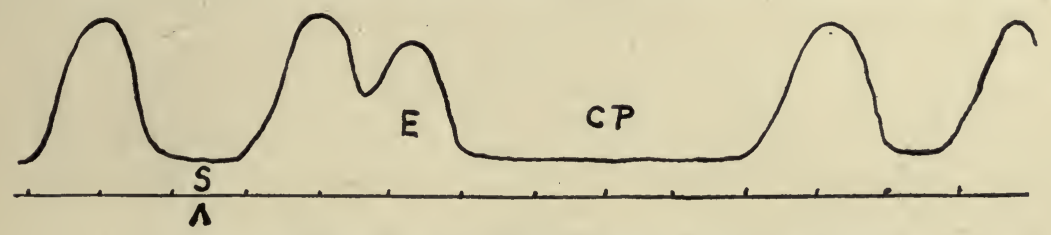

Fig. 52.-Stimulation of Frog's Heart During Diastole.

$S$, Moment of stimulation; $E$, extra contraction; $C P$, compensatory pause.

4. The Transmission of the Wave of Excitation.-The wave of excitation, ordinarily started at the venous entrance of the heart, is transmitted in the turtle and frog over muscular connections and activates its different segments consecutively. The transmission of this impulse may be interfered with by compressing these muscular bridges by means of a screw-clamp applied to the auriculoventricular junction. By gradually tightening this screw a degree of compression may be established which will allow only some of these waves of excitation to reach the 
ventricle. Not all of the auricular contractions will then be followed by ventricular contractions. This constitutes the condition of partial heart-block.

5. The Excised Heart.-Raise the heart as a whole by the frenum, and divide the aortæ and venæ cavæ. Place the excised organ in a watch-glass in some of its blood and cover it with another watch-glass. Count the number of its beats, and note that it becomes soft and flaccid during diastole and adjusts itself at this time to the surface upon which it rests. Apply a drop of warmed saline solution to the sinus. Note the increase in the rate of this segment, which, in turn, brings about a similar change in the other portions of the heart.

6. Isolated Segments of the Heart.-The fact that a heart when separated from the central nervous system continues to beat, shows that it is automatically active. Cut transversely across between the sinus venosus and the auricles. The sinus continues to beat, whereas the rest of the heart ceases to beat for a time. This proves that the auricles and ventricle are ordinarily activated by a wave of excitation derived from the sinus. After a time the severed portion of the heart develops a beat of its own and continues to contract rhythmically.

Sever the ventricle from the auricles. . It ceases to beat, but may be made to contract at any time by stimulating it, for example, by pricking it with the point of a scalpel. It usually executes several beats.

Cut off the apex of the ventricle, but preserve its basal portion. The former remains quiescent, but reacts promptly to all stimuli. Since the apex is free from nervous elements, this experiment is usually cited to prove that the heart-beat is of myogenic origin.

7. All-or-none Law.-Suspend this preparation of the apical portion of the ventricle by means of two silk threads between the writing lever and the plate. Apply the hand-electrodes to it and stimulate it successively with single induction shocks of different strength. Do you observe a difference in the amplitude of its contractions? How does striated and non-striated muscle tissue behave under these circumstances? Inquire into the reason for this difference.

8. Isolated Strips of Ventricle.-Fasten one pole of the quiescent basal portion of the ventricle to the hook of a weight resting upon the bottom of a beaker, and its upper pole to the hook and string of a writing lever. Counterpoise, so that this preparation is under the least possible tension. Pour a solution of 0.7 per cent. sodium chlorid into the beaker until it fully covers this preparation. Wait until the latter shows continued activity (twenty to thirty minutes), and record successive series of contractions at intervals of ten minutes. When the contractions have weakened, add a few drops of a 1 per cent. solution of calcium chlorid to the saline. The calcium stimulates cardiac muscle (systole), and hence the individual contractions should again assume their former amplitude.

In small doses potassium favors the relaxation of cardiac muscle, while in larger doses it brings about a continued diastole. To show this 
effect, it may suffice to add several drops of a 0.9 per cent. solution of potassium chlorid to the saline solution. As soon as the preparation has become quiescent, immerse it in fresh saline solution or in Ringer's fluid, which contains the aforesaid salts in the following proportion: $\mathrm{NaCl}, 0.7$ per cent.; $\mathrm{KCl}, 0.035$ per cent.; $\mathrm{CaCl}_{2}, 0.026$ per cent. The rhythm will presently be restored.

Annotation.-With some care a turtle's heart may be made to last throughout these experiments. If it does not, use a frog's heart to complete this series. The action of the salts may also be studied separately upon different hearts. The preceding order, however, should be adhered to, owing to the possibility of saving material.

Conduction Through the Ventricle.-Pith a frog and expose the heart. Destroy the continuity of the nerve-fibers in the ventricle by making four interdigitating cuts across it-two cuts starting from its left border and two from its right. Since the wave of contraction nevertheless descends over this zigzag strip, the wave of excitation must be propagated by the muscular elements.

Separate the ventricle from the auricles by a transverse cut. Note that the latter continue to beat synchronously with the sinus, while the former remains quiescent. Apply the hand-electrodes successively to the base and apex of this zigzag strip of ventricle. Observe that the wave of contraction can thus be made to travel from base to apex as well as in the reverse direction. 




\section{LESSON XIV}

\section{THE HEART (Continued)}

\section{INHIBITION AND ACCELERATION OF THE SIMPLE HEART. ACTION OF NICOTIN, ATROPIN, AND MUSCARIN}

1. Inhibition of the Heart.-Apply a ligature tightly to the neck of an etherized turtle and destroy the brain by pithing. Remove the ventral shield or plastron and clip away the projecting angles of the shoulder-blades. Arrange the inductorium for stimulation with a tetanic current of medium strength. Hold the extended neck of the turtle in place and carefully isolate the vagus nerve on each side. Place each in a loose ligature. Open the pericardial sac and connect the apical band of connective tissue with the writing lever. Adjust the

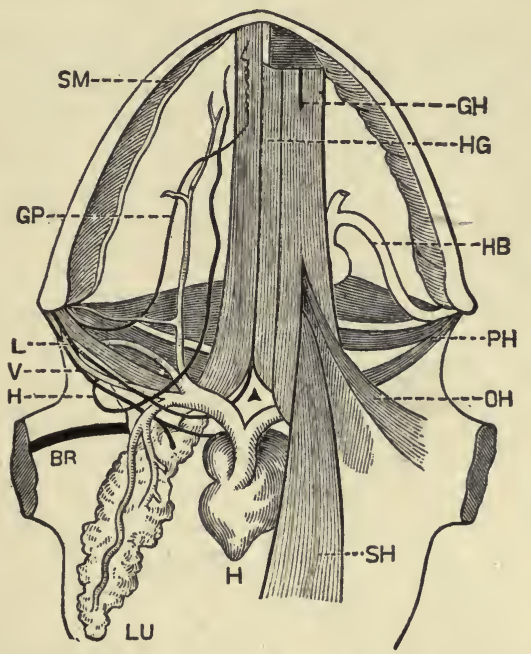

Fig. 53.-Course of Vagus Nerve in Frog. (Stirling.)

$S M$, Submentalis; $L U$, lung; $V$, vagus; $G P$, glossopharyngeal; $H S$, hypoglossal; $L$, laryngeal; $P H, S H, G H, O H$, petro-, sterno-, genio-, and omohyoid; $H G$, hypoglossus; $H$, heart; $B R$, brachial plexus.

writing point of a chronograph underneath the writing point of the lever. Raise the left vagus nerve and place it upon the electrodes. Having recorded a number of normal heart-beats, stimulate the aforesaid nerve. If the heart is not inhibited, increase the strength of the current, but not excessively, because an electrolysis might then result which would destroy conduction permanently.

Make a number of these records, stimulating each time for a few seconds. During what period of the cardiac cycle is the heart arrested? 
Study the character of the heart-beats occurring directly after the inhibition. They may be small at first and gradually become larger, or large at first, and slowly decrease to normal. No explanation can be given for this difference.

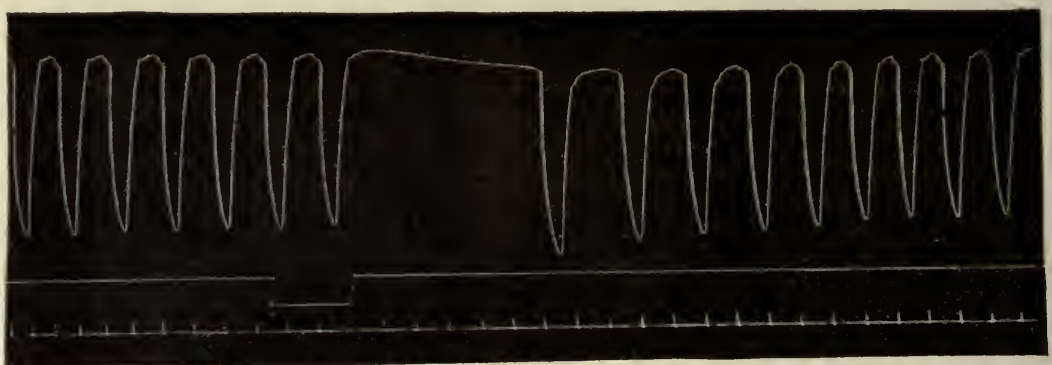

Fig. 54.-Record of the Contractions of the Frog's Heart During Stimulation of the Vagus Nerve (Tension Lever).

The time is given in seconds, the stimulation is indicated by the signal.

Stimulate the right vagus nerve with the same strength of current. Do the nerves possess the same inhibitory power? They differ, but not always in the same manner, $i . e$., the left or the right nerve may be more powerful, but most generally the right. The same differences may be

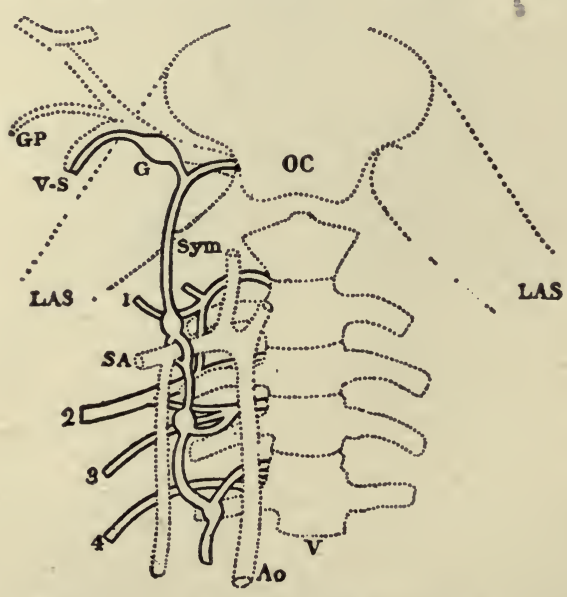

Fig. 55.-Schema of the Sympathetic Nerve in the Frog.

$O C$, Occiput; $L A S$, levator anguli scapulæ; $S y m$, sympathetic; $G P$, glossopharyngeus; $V-S$, vagosympathetic; $G$, ganglion of the vagus; $A o$, aorta; $S A$, subclavian artery. (Stirling.)

noted in the mammals. Occasionally a turtle will be found in which neither vagus possesses this function.

Stimulate either vagus nerve for three or four minutes. Note that the heart "escapes" from the inhibition in a very short time, and subsequently continues to beat in spite of the stimulation. 
Tighten the ligatures upon both vagi nerves. Apply a second ligature to each at a distance of $2 \mathrm{~mm}$. from the first. Cut between them. Stimulate both central ends successively. Do you observe a change in the character of the record now made? Stimulate both distal ends successively. Do you observe a difference between this record and that obtained previously with the intact nerve?

Apply the electrodes transversely to the sino-auricular region of the heart. Stimulate after you have recorded a limited number of normal beats. Does the inhibition produced in this way differ from that previously obtained by stimulating the vagi nerves?

Annotation.-A very convenient way is to trephine the ventral shield of the turtle in the region of the heart. 'This saves much labor and prevents loss of blood and drying of the tissues. Moreover, if small pulley-wheels are at the disposal of the students, the heart should be allowed to act in its normal horizontal position, while the string is made to move across the pulley, placed obliquely below the writing lever. Do not allow the heart to act under too great a tension and allow it to rest from time to time by disconnecting the string. In case it should cease contracting properly, apply a few drops of warmed saline solution to its surface. Arhythmias are not uncommon and may be remedied in just this way.

2. Action of Nicotin.-By means of a dropper apply a small quantity of a 0.2 per cent. solution of nicotin to the heart. After five minutes connect its apex with the writing lever and stimulate the vagus nerve. The heart is not inhibited. Stimulate the sino-auricular region directly. The heart is inhibited. What is your conclusion regarding the action of nicotin?

Annotation.-Nicotin is a nerve-cell poison affecting the neuron at the synapse. In this case it causes a break between the vagal terminals and the recipient cells of the inhibitor plexus (Remack's), situated in the region of the sino-auricular groove. Consequently, the inhibitor impulses set up by stimulating the vagus can never reach the postganglionic path and effector. The stimulation of the plexus itself remains effective, because the postganglionic elements are thereby excited directly.

3. Action of Atropin.-Apply to the heart of the same turtle a few drops of a 0.5 per cent. solution of atropin sulphate. After a few minutes stimulate the plexus situated at the sino-auricular junction. Observe that this stimulation now fails to inhibit the heart. Explain.

Annotation.-This experiment should, of course, be performed upon a fresh turtle. It will then be found that the atropin destroys the inhibitor power of the vagus as well as that of the plexus at the sino-auricular groove. This result is due to its paralytic effect upon the distal terminals of the postganglionic fibers. Atropin is primarily a nerve-fiber poison. Since in the above experiment the stimulation of the vagus has already been rendered ineffective by the nicotin, this effect cannot be noted. It is for this reason that a fresh turtle should be used for the atropin. With a class of, say, 100 students this would entail a considerable expense which may be avoided by supplying this information or by permitting one group of students to perform the experiment with nicotin, and another the experiment with atropin.

4. Action of Muscarin.-Apply to the ventricle of the same turtle a few drops of normal saline to which a little muscarin has been added. 
A 1.0 per cent. solution of pilocarpin acts in the same way as muscarin. Note the gradual inhibition of the ventricle.

Annotation.-It will be remembered that this poison produces its characteristic effect either by paralyzing the contractile elements of the muscle-fibers or by stimulating the inhibitor nerve mechanism. The latter view seems the more plausible, because if a few drops of a 0.5 per cent. solution of atropin are applied to a heart which has been arrested with muscarin, this organ resumes its beat. Atropin acts antagonistically to the muscarin by counteracting the effect of the latter.

5. Reflex Cardiac Inhibition.-Etherize a frog and lay it upon its back. Make a rounded orifice in the ventral surface of the thorax in the region of the heart so as to expose this organ fully to the view. With the flat handle of a scalpel continue to tap lightly upon the ventral aspect of the abdomen until the heart shows a material reduction in its frequency. Explain this result, comparing it with the symptoms following strokes upon the solar plexus in man.

Divide both vagi nerves and repeat this experiment. Note that the aforesaid procedure now fails to inhibit the heart, because the paths by means of which these impulses reach the heart have been cut.

Annotation.-In the frog the vagi nerves are not easily found. Insert a glass rod in the esophagus to distend it. Remove the tissues over the petrohyoid muscle, extending from the angle of the jaw to the thyroid process of the hyoid bone. Two nerves will be seen pursuing a course across this muscle, namely, the hypoglossal and the glossopharyngeal. The first is easily recognized by tracing its course to the tongue. It lies closer to the midline. Next to the lower border of the petrohyoid muscle and close to a blood-vessel lies the vagus (Fig. 53).

6. Acceleration of the Heart.-Arrange the apparatus for registering the contractions of the frog's heart upon the smoked paper of a moderately rapid kymograph. Pith a frog and expose the heart by making a median incision through the wall of the thorax. Expose the spinal column at the base of the skull by pushing the esophagus and trachea to one side. Identify the vagus ganglion. It lies under the upper part of the levator scapulæ muscle. After its formation the sympathetic nerve turns outward along the base of the jaw to become united with the vagal fibers. Black pigment marks the course of this nerve. Isolate this portion and insulate it with a narrow strip of rubber membrane. Allow the heart to register its beats while the drum revolves once around its axis in about one minute. Add the record of a chronograph. Directly underneath register a second line of heart-beats, but stimulate the sympathetic nerve during this entire period with a tetanic current of very moderate intensity. Count the beats in each line, and compare. The stimulation of this nerve in the frog does not produce a very decisive acceleration; still, records of this length should show a difference of from ten to fifteen beats. 




\section{LESSON XV}

\section{THE HEART (Continued)}

\section{STANNIUS' EXPERIMENT. STAIRCASE PHENOMENON. SUMMATION OF STIMULI. ACTION OF THE CONSTANT CURRENT, ETHER, AND CHLOROFORM. DISSECTION OF THE MAMMALIAN HEART}

1. Stannius' Experiment.-Pith a frog and expose the heart by a median incision through the wall of the thorax. Use two thin silk threads. Place one around the sino-auricular groove, and tighten it moderately until the auricles and ventricle cease beating. Apply a second ligature to the auriculoventricular groove, and tighten it sufficiently until all three parts of the heart beat again. Explain this phenomenon.

2. Staircase Phenomenon.-Remove the ligature previously applied to the auriculoventricular groove, so as to render the auricles and ventricle again quiescent. Connect the latter with a light heart lever (suspension method), and stimulate its substance with single induction shocks at intervals of five seconds. Each time move the stationary drum a short distance. The heart usually reacts to stimuli of different strengths by giving maximal contractions, but its amplitude of reaction is determined chiefly by its condition. In the Stannius preparation certain conditions have arisen which convert the first four or five contractions into an ascending series.

3. Summation of Stimuli.-The Stannius preparation may also be made to show the phenomenon of summation of stimuli. To accomplish this end separate the secondary coil from the primary until the break shock just ceases to be effective. Stimulate the heart with this subminimal stimulus in quick succession until a contraction is obtained.

4. Incomplete Tetanus.-The Stannius preparation may also be made to yield an incomplete tetanus by passing a quickly interrupted current through it.

5. Effects of the Constant Current.-Remove the ligature which has previously been applied to the sino-auricular groove. The heart should resume its normal beat. If not, use a fresh preparation. Insert in the frog's mouth the wire connected with the zinc of a dry cell (cathode). Place the flat end of the wire attached to the carbon (anode) upon the surface of the ventricle. As the well-filled ventricle contracts, that portion of it which rests against the anode will remain relaxed and present a flushed appearance. In this way a local diastole is produced in a general field of systole. Suddenly remove the wire, breaking the circuit. The area just alluded to now remains contracted, and shows, therefore, a pale appearance. A local systole arises within a field of general diastole. This experiment may be employed to show that the anode depresses on the make, but stimulates on the break. 
The wires should now be reversed to show the cathodal excitation on the making and the cathodal depression on the breaking of the constant current.

6. Action of Chloroform and Ether.-Excise two frogs' hearts and place each in a watch-glass. Cover them equally with a sufficient quantity of saline solution (5 c.c.). Add a drop of pure chloroform to one and a drop of ether to the other. Note that the heart in the former solution loses its tonus rapidly and soon ceases to beat, while the action of the other does not seem to have been materially impaired. In order to produce the same effect with the ether, as many as 10 drops might have to be added to the original solution.

7. Dissection of the Mammalian Heart.-Procure the heart of an ox or sheep. Ask to have the "tubes cut long." Identify its different parts and test the relative thickness of the walls of its chambers. What is the functional significance of this difference in the volume of the muscle substance? Identify the aorta, pulmonary artery, venæ cavæ, and pulmonary veins. Note the appearance and strength of the walls of these blood-vessels. Trace the coronary arteries from the arch of the aorta and identify the coronary sinus.

Explore the auricles and ventricles by inserting the index-finger through the stumps of the different vessels. Palpate the interauricular septum and locate the fossa ovalis. Slit open the two auricles and examine their walls and recesses. Note that the venous orifices are not guarded by valves.

Beginning at the apex of the heart make a series of transverse sections until both ventricles have been opened. Note that the left cavity is incised first and possesses a much thicker wall than the right. Obtain an unobstructed view of the tricuspid and mitral valves by making a longitudinal incision through the wall of each ventricle. Study the appearance and distribution of the papillary muscles, columnæ carneæ, moderator bands, and chordæ tendineæ. Approximate the valve flaps and note the manner of insertion of the chordæ tendineæ. Trace the course of the bundle of His.

Tie a short glass funnel in the stump of the ascending aorta. Hold the heart vertically and pour water into the funnel. Observe that the semilunar valve closes, thereby preventing the water from escaping into the left ventricle. By means of a long probe push one of the flaps aside. Note the rush of water into the ventricle. This condition represents aortic insufficiency (regurgitation). Repeat the experiment on the right side. The sinuses of Valsalva are here more prominent, because the vessel wall is much thinner. 




\section{LESSON XVI}

\section{THE HEART (Continued)}

\section{THE BEATING MAMMALIAN HEART. HEART-BLOCK. FIBRILLATION}

1. The Beating Mammalian Heart in Situ.-Test the artificial respiration apparatus to see whether everything is in proper working condition. Have the ether bottle and connecting parts ready for use. Etherize a cat and maintain deep anesthesia throughout these experiments and until the animal has been killed.

Perform tracheotomy in accordance with the directions given on page 67. Make a median incision through the skin covering the sternum. Cut through the ensiform cartilage and median line of the sternum, taking special care not to divide the mammary arteries. Institute artificial respiration, adjusting the volume of air so as to give a normal degree of expansion to the lungs. Stop bleeding by torsion

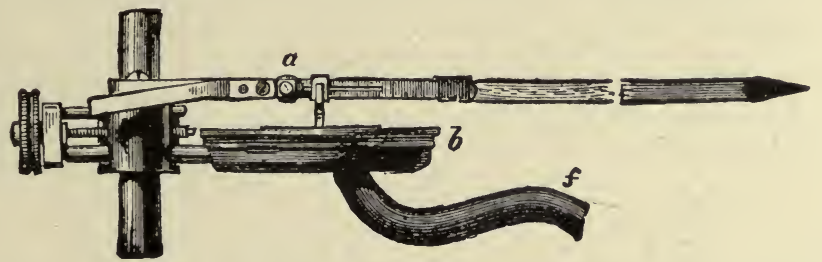

Fig. 56.-Marey's Tambour.

$a$, Axis of lever; $b$, metal tray covered with rubber membrane, and communicating by tube $f$ with the cannula.

and ligation of the vessels. Separate the walls of the thorax by means of a string drawn around the board. Identify the pericardium and large blood-vessels leaving and entering the heart.

Insert the end of a small cannula through an opening in the pericardial sac, and secure it by means of a ligature. Connect its free end by means of narrow rubber tubing with a recording tambour adjusted against the paper of a kymograph. The pericardial sac acts in this case as a plethysmograph and yields a tracing of the volumetric differences which the heart displays during its cycle. Detach the rubber tube from the recording drum and blow a small quantity of air into the pericardial sac. What influence does the increase in intrapericardial pressure exert upon the activity of the heart? Note that this procedure produces dynamic conditions such as are found in pericarditis.

Open the pericardial sac widely and reflect the pericardium upward. Note the character of the fluid escaping through the incision. What is its function? Identify the different parts of the heart. Which side of 
the heart is more fully exposed to the view when the chest is opened? Palpate with the tip of your index-finger the walls of the right and left ventricles, observing in each case the difference in the texture of the cardiac muscle on systole and diastole. Also note the much greater thickness of the left musculature. Observe that the two auricles contract practically simultaneously, and that their contraction begins near the orifice of the venæ cavæ. Likewise, note that the two ventricles contract together as soon as the auricular systole has been completed. Expose the superior and inferior cavæ and observe their pulsations (venous pulse). Expose the aorta and pulmonary artery and study their pulsations (arterial pulse).

Place the tip of your index-finger upon the surface of the left auricular appendix. Press downward. Do you feel the flaps of the mitral valve hitting against your finger? Repeat this observation on the opposite side. Blow a spray of albulin upon the heart to prevent its drying.

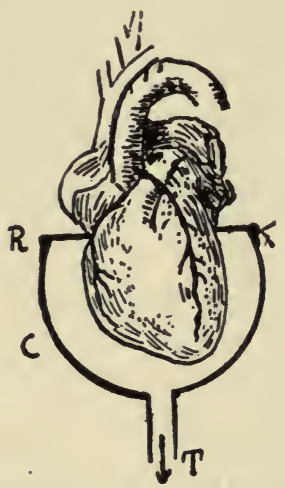

Fig. 57.-Cardiometer.

The heart is inserted through a perforation in rubber membrane $(R)$ into cavity of a hemispheric glass capsule $(C)$. The latter is connected with a recording tambour $(T)$.

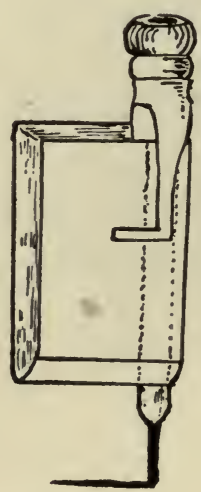

Fig. 58.-Clamp for Pronucing HeartBLOCK. (After Erlanger.)

Discontinue the artificial respiration for a brief period of time until the heart has been markedly slowed. Again study the progress of the wave of contraction and note the gradual change in the color of the heart. Its venosity is most clearly betrayed by the left auricle, the color of which gradually changes from bright red to dark purple. Explain this phenomenon. Resume artificial respiration. Allow a few drops of a slightly warmed saline solution to fall upon the heart. Note the increase in its frequency.

Procure a small cardiac plethysmograph, usually consisting of a hemispheric capsule of glass the orifice of which has been closed with a rubber membrane. Incise the rubber membrane near its center, and push the ventricular portion of the heart through this opening into the cardiometer. Connect the tubular outlet of the latter with a recording drum 
and again register the volumetric changes of the heart. Occlude the venæ cavæ for a few moments. Explain the result. Withdraw the ventricles from the cardiometer and allow the heart to recuperate. If necessary, cover it for a time with cotton moistened with warmed saline solution.

Again record the volume-curve of the ventricles. Temporarily obstruct the arch of the aorta. Explain the result. Allow the heart to rest.

2. Heart-block. Fibrillation.-Procure a clamp such as is represented in Fig. 58. Remove the loose connective tissue from the wall of the aorta along a line forming the right edge of the mass of fat which covers the anterior aspect of this blood-vessel. Set the point of the hook in this place, corresponding to the tip of the membranous septum of the ventricles. Direct the needle obliquely downward and toward the lumen of the aorta and force it into the left ventricle, approximately at the junction of the right and posterior flaps of the mitral valve. Turn the point of the hook backward, so that the short arm of the L-shaped clamp comes to lie parallel to the ventricular septum. Now, move the bar until it lies parallel to the first part of the arch of the aorta and force the short arm of the hook into the septum at a point about 3 to $6 \mathrm{~mm}$. below the auriculoventricular junction and somewhat posterior to the mesial flap of the mitral valve. Do not include the main trunk of the coronary artery in the clamp. Tighten the clamp sufficiently to cause a partial block. Not every auricular contraction will then be followed by a ventricular contraction. This condition simulates the condition of heart-block in man, usually brought about by inflammatory lesions along the course of the bundle of His.

If done in an improper manner, the ventricle will immediately be thrown into a condition of fibrillation. Its musculature then executes irregular wave-like contractions. This condition may also be produced by injections of strophanthin (about 0.0003 gram). Shortly before the onset of the fibrillation the heart frequently shows a typical block. 





\section{THE HEART (Concluded)}

\section{PERCUSSION AND AUSCULTATION OF THE HUMAN HEART UNDER DIFFERENT CONDITIONS}

1. The Area of Cardiac Dulness.-Percuss the region of the heart of the subject, beginning in each case well without the boundaries of this organ, and passing radially toward the sternal articulation of the third rib. Accurately note the point where the deep resonance of the lung passes, over into the area of dulness of the heart. Outline the boundaries of the latter with colored chalk. What is its size and position? How is this area changed by the movements of the lungs on inspiration and expiration? Explain. If abnormalities in its size are found, give probable cause. Outline the area of cardiac flatness.

Annotation.-Percussion is usually practised by placing the middle and indexfingers of your left hand flat against the wall of the chest. Tap upon them sharply with the index and middle fingers of your right hand. In tapping, the right hand should be held lose at the. wrist, and the second and third phalanges should be flexed upon the first at right angles.

2. The Rate of the Heart.-Determine by auscultation the number of the heart-beats of the subject while he successively assumes the recumbent, sitting, and standing position. Tabulate the results and ascertain the differences. Have the subject make forty flexions and extensions of the arms in one minute. Again determine the cardiac rate. Repeat the count after the subject has made thirty deep knee bendings in one minute, and again after a stationary run lasting onehalf minute. Arrange your results in the form of a table.

Determine the heart-rate for one minute. Ask the subject to swallow. What difference do you note?

Determine the heart-rate. Ask the subject to concentrate his attention upon his heart. Do you note any difference? Ascertain whether the subject is able to increase his cardiac rate volitionally.

Annotation.-The method of auscultation is conveniently practised by applying the ear to the chest of the subject, preferably in the region between the left nipple and the sternum. The sounds are loudest in this area. Since we are dealing in the above experiments with the frequency of the heart, you may allow a thin garment to intervene between your ear and the skin of the subject.

3. The Cardiac Impulse or Apex-beat.-Carefully observe the chest in the region of the apex of the heart, $i . e$, in the fifth intercostal space, and at a distance of about $2 \mathrm{~cm}$. to the right of the left nipple. Note the periodic protrusion of the chest wall. Mark the location of this 
impulse with a blue pencil. Observe the changes in its conspicuousness when the position of the subject is changed and during inspiration and expiration. Explain the results. If the impulse is not in its normal place, ascertain the direction and degree of its displacement. Give its probable cause. Palpate the radial artery and note the interval of time between the radial pulse and the cardiac impulse. Palpate the carotid artery low in the neck. Note that the difference in time between this

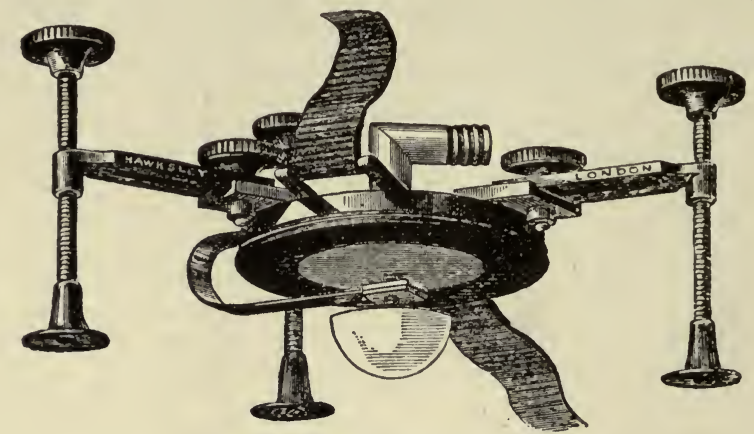

Fig. 59.-CARDIOGRAPH.

The tape is strapped around the chest. The central button is applied to the "apexbeat" and its pressure on the chest wall regulated by means of the three screws at the sides. The tube at the upper part of the instrument serves to connect the drum of the cardiograph with a registering tambour. (Sanderson.)

pulse and the cardiac impulse is much less, a result easily explicable upon the ground of distance.

Adjust a cardiograph to the area of the cardiac impulse. Connect it with a recording tambour and allow the latter to register its excursions upon the smoked paper of a kymograph revolving at a moderate speed. This record should be made above a time-curve, registered by a Jaquet chronograph.

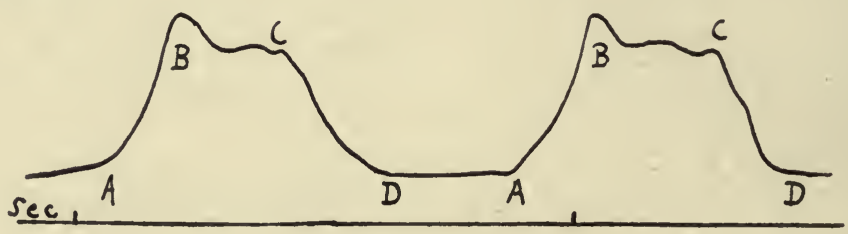

Fig. 60.-Cardiogram.

$A B$, Systole; $B C$, plateau; $C D$, diastole; $D A$, pause; time in seconds.

Explain the character of the cardiogram so obtained. Excepting the cardiac rate, is it possible to derive valuable data from this curve which more particularly pertain to the quality of the contractions of the heart? Give reasons for your conclusions.

Annotation.-The method of palpating the radial artery is practised as follows: With your left hand support the right hand of the subject in a position of slight extension. As a rule, the right artery gives better results. Place the second and 
third fingers of your right hand upon the radial artery close to the wrist. It is then a simple matter to determine the frequency of the pulse. Besides, the third finger may be employed to compress the artery and the second to palpate. In this way the experimenter may obtain an idea regarding the tension prevailing in the vascular system of the subject.

4. The Heart Sounds.-Adjust your ear directly to the area of the apex-beat of the subject. Study the quality and intensity of the cardiac sounds. How many sounds do you hear? Note the pause following the second sound. Palpate the radial pulse and determine whether these sounds are produced during ventricular systole or diastole. Ascertain precisely when they occur and where they are most intense. Request the subject to stop respiring after a deep inspiration and observe the lesser audibility of these sounds. State the reason for this change. Ask the subject to cease respiring after a forced expiration. Why are these sounds now more clearly heard?

Repeat these tests with the help of a stethoscope. Place its chestpiece over different regions of the heart. Compare the relative intensity of the sounds when heard at the apex and when heard over the junction of the second right costal cartilage. Do you obtain a difference? Give reasons for it.

Having thoroughly familiarized yourself with the character of the normal heart sounds, study a simple murmur, such as may arise in consequence of mitral stenosis or aortic regurgitation. Note its character, point of greatest intensity, and relation to the cardiac cycle. Also familiarize yourself with the so-called extracardiac friction sounds, hemic murmurs, and arterial and venous bruits. 

- 



\section{THE CIRCULATION}

\section{THE CAPILLARY CIRCULATION. CONVERSION OF AN INTERMITTENT} INTO A CONSTANT FLOW. SCHEMA OF THE CIRCULATION

1. The Capillary Circulation.-Procure a microscope with low- and high-power objectives, a thin cork board about $20 \mathrm{~cm}$. long and $10 \mathrm{~cm}$. wide, and a few pins. Bore a hole about $1 \mathrm{~cm}$. in diameter close to the margin of the cork board. Pith a frog, being careful not to lose any blood. Immediately close the opening with the pointed end of a short piece of wood. Place the frog, dorsum turned upward, upon the board and bring one foot over the hole in the cork board. Stretch the web uniting the second and third toes across the opening, and hold the toes in place by means of pins or threads. Do not stretch the web unduly, so as not to block the blood-vessels. Adjust the body of the frog in an easy position.

Fasten the cork board to the stage of the microscope and illuminate the web under an objective of low power. Moisten the frog repeatedly with saline solution. Observe the movement of the blood and differentiate between the red and white corpuscles. Note the differences in the caliber of the blood-vessels and the speed of the blood flow. Ascertain whether a certain vessel is an arteriole, a true capillary, or a venule. Select a true capillary and observe how the red cells force their way through it, elongating if necessary. Find a capillary which is so small that only plasma passes through it. Do you observe an intermittency in the flow anywhere in the field? What is its probable cause? Have you seen a reversion of the blood flow? Give its cause.

Carefully adjust the high-power objective to the web, and repeat the preceding observations.

2. Intermittent, Remittent, and Constant Flow.-Procure a piece of band-tubing about $1.5 \mathrm{~m}$. in length. Insert in one of its ends a glass cannula, and in the other a valved rubber syringe. Dip the inlet tube of the latter in a basin of water and ask the assistant to hold the end of the band-tubing over the sink.

Compress the rubber bulb at intervals of five seconds. Note that the tubing fills gradually, but does not discharge until it has attained a definite degree of distention. It then discharges a certain quantity of water with every compression of the bulb (intermittent flow). Compress at a faster rate until the flow does not cease entirely during the interims (remittent flow). Compress at still briefer intervals until the tubing attains a high degree of distention and continues to empty its contents evenly during the time when the rubber bulb aspirates (constant flow). Obviously, the successive muscular efforts required to 
compress the rubber bulb are stored in the wall of the tubing in the form of elastic tension. As soon as the internal pressure is lessened during the interims this elastic power acts upon the water within the tubing and continues to force it onward through the outlet. To accomplish this end the tubing must be highly distended, $i$. e., it must be

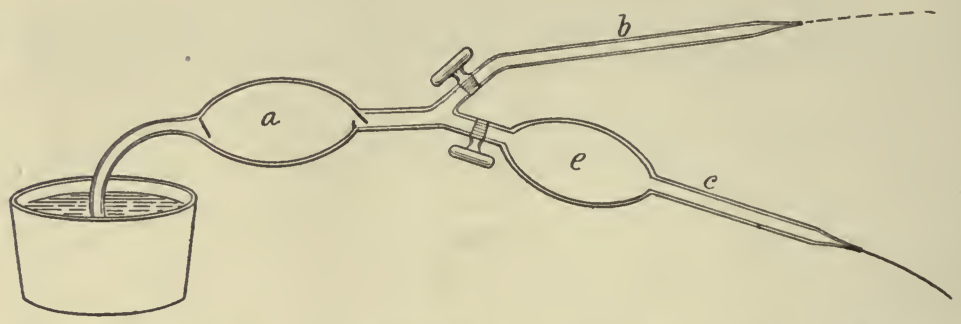

Fig. 61.-Simple Schema to Illustrate the Factors Producing a Constant Head of Pressure in the Arterial System.

$a$, A syringe bulb with valves, representing the heart; $b$, glass tube with fine point representing a path with resistance alone, but no extensibility (the outflow is in spurts synchronous with the strokes of the pump); $c$, outflow with resistance and also extensible and elastic walls represented by the large rubber bag, $e$, the outflow is a steady stream due to the elastic recoil of the distended bag, $e$. (Howell.);

retained in a condition of hyperfilling. To what constituents of the vascular system may the different parts of this apparatus be compared?

Remove the narrow glass cannula (capillaries) from the end of the band-tubing (arteries). This decreases the peripheral resistance. What effects do you observe? Insert a narrow glass carnula and note the effects of this increase in the peripheral resistance upon the distention

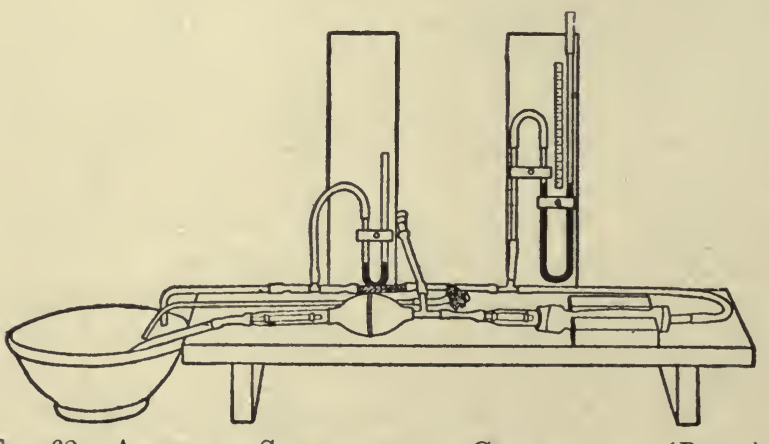

- Fig. 62.-Artificial Schema of the Circulation. (Porter.)

of the band-tubing and the escape of water. In the latter case you may materially decrease the number of the compressions without destroying the constancy of the flow.

3. Schema of the Circulation.-The basin of water represents the left auricle, the rubber bulb the left ventricle, and the large rubber tube the aorta. The large glass tube next to the basin contains the mitral 
valve, and the one distally to the bulb the aortic semilunar valve. The side branch from the ventricle may be connected by means of a thistle tube with a membrane manometer, and may thus be used to register the changes in pressure in the ventricle. In a similar manner the changes in pressure in the aorta may be recorded (arterial pulse). Beyond the aorta lies the remaining portion of the arterial system, to which there is attached a mercury manometer for measuring the arterial pressure. Into the dish opens the venous system. It is equipped with a mercury manometer for measuring the venous pressure. Between the arteries and veins are the capillaries, represented in the schema by a short piece of porous bamboo, and a side branch bearing a clamp.

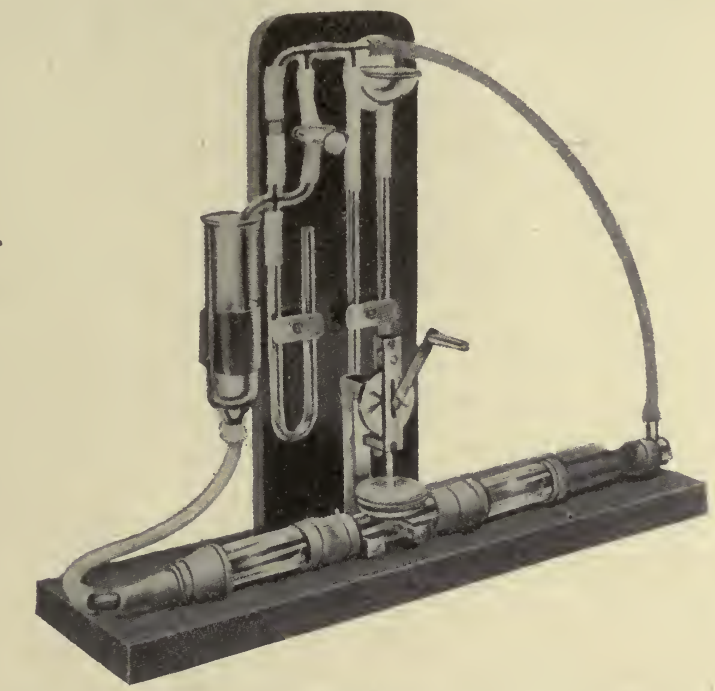

Fig. 63.-More Recent Schema of the Circulation.

The action of the heart is here imitated by a tambour, rhythmically compressed by hand. The veins empty their contents into a receptacle (right auricle) fastened to the side of the upright stand. (Harvard A pparatus Co.)

A. Normal Circulation.-Dip the inlet and outlet tubes of this system into the water in the basin and pump gently with the rubber bulb until the different tubes have been filled with water. Clamp the side branch between the arteries and veins. Pump gently at the rate of about sixty times in a minute, and observe the following:

(a) The action of the valves,

(b) The arterial pressure and its changes,

(c) The venous pressure,

(d) The pulse in the aorta, and

(e) The character of the flow from the veins.

Open the clamp slightly, so as to simulate dilatation of the arterioles, and pump as before. What is the effect of this procedure on the arterial and venous pressures and on the character of the venous flow? 
Open the clamp widely, and pump at the rate of about twelve times in a minute. Effect?

Determine the respective conditions under which the venous flow becomes intermittent, remittent, and constant.

Try to discover the causes, in the normal circulation of the blood, of the great difference between the arterial and venous pressures, and the absence of a pulse in the veins.

B. Intraventricular Pressure.-Place a thistle tube against the membrane closing the lateral tube of the rubber bulb (ventricle) and connect it with a recording drum. Register the changes in pressure occurring within the ventricle upon the smoked paper of a slowly revolving kymograph.

C. Pulse.-Place the thistle tube upon the "aorta" and register its pulsations.

D. Mitral Insufficiency.-Remove the rubber sheath from the glass tube representing the mitral valve. This produces a condition analogous to mitral insufficiency. Pump at a normal rate. Observe the effect of this lesion upon the intraventricular pressure, the arterial pressure, the aortic pulse, and the venous discharge. Replace the sheath.

E. Mitral Stenosis.-Tie a thread around the sheath of the mitral valve, thereby restricting the size of this opening. This produces a condition analogous to mitral stenosis. What effects do you notice?

F. Aortic Insufficiency.-Remove the sheath from the semilunar valve, producing thereby a condition analogous to aortic insufficiency. What changes do you observe? Replace the sheath.

G. Aortic Stenosis.-Tie a thread around the sheaih of the semilunar valve so that the opening becomes smaller. This condition simulates aortic stenosis. Observe the results. 




\section{THE CIRCULATION (Continued)}

\section{THE CAUSE AND VELOCITY OF THE PULSE. DIRECT METHOD OF ASCERTAINING THE BLOOD-PRESSURE}

\section{Schema Illustrating the Differences in the Velocity of the Blood} Flow.-Procure a glass bulb, such as is represented in Fig. 64. Connect its inlet tube by means of a relatively narrow glass tube with the water hydrant. On opening the stop-cock the water will advance to a higher level, its flow being most rapid in the narrow inlet tube, very slow in the enlarged central portion, and intermediate in the outlet tube. If all the arteries, capillaries, and veins could be united into single tubes, the calibers of these three divisions would differ in the same way, $i$. e., the bed formed by the arteries would be the smallest of all, and that of the capillaries the largest. Since the speed of flow is inversely proportional to the lumen of the vessel, the blood must attain its greatest velocity in the arteries, and its slightest velocity in the capillaries.

2. Schema Illustrating the Cause and Velocity of the Pulse.-Procure four glass tubes, each about $1 \mathrm{~m}$. in length and $1 \mathrm{~cm}$. in diameter. Arrange them vertically about $20 \mathrm{~cm}$. apart, and connect them in series by means of bandtubing, each piece being about $50 \mathrm{~cm}$. in length. Attach a valved rubber bulb to the end of this system, and allow its inlet tube to dip into a basin filled with water. Compress the bulb

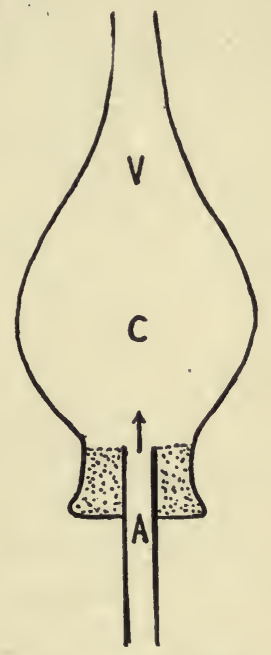

Fig. 64.-Schema IllustratiNG THE DIFFERENCE IN THE VELOCITY OF THE BLOOD FLOW. $A$, Arteries; $C$, capillaries; $V$, veins. rhythmically until this entire system has been well filled. The water then rises in the laterals to a certain level, indicating the pressure existing at these different points. Secondly, each compression of the bulb then produces an oscillation of the water in the successive laterals, the water in the lateral nearest the rubber bulb being moved first (Fig. 65).

3. The Direct Method of Determining the Arterial Blood-pressure. -Weigh the animal and compute the quantity of blood present in its body. Give it ether and maintain the anesthesia until it has been killed. Perform tracheotomy. Expose the carotid artery on the left side and the external jugular vein on the right side. (See Lesson X.) Place a silk thread loosely around each blood-vessel. Raise both sufficiently to occlude them. Notice that the artery is more highly distended on the 
central side of the ligature, and that its distal end does not collapse altogether, owing to the fact that the carotid arteries anastomose rather freely with one another. Observe that the distal end of the vein is highly distended, whereas its central end is collapsed. This is one of the tests which Harvey employed to prove the circulation of the blood.

Observe the changes in the caliber of the vein during inspiration and expiration. During the former period its size is decreased, and during the latter, increased. These changes are referable to the aspiratory action of the thorax, because the inspiratory movement augments the elastic pull of the lung tissue upon the yielding walls of the central venous trunks. During expiration, on the other hand, the lung tissue recoils more completely, and does not exert so powerful a traction upon the venous trunks and their contents. Accordingly, the inspiratory movement decreases the pressure in the central veins and draws a larger quantity of blood into them and the right side of the heart.

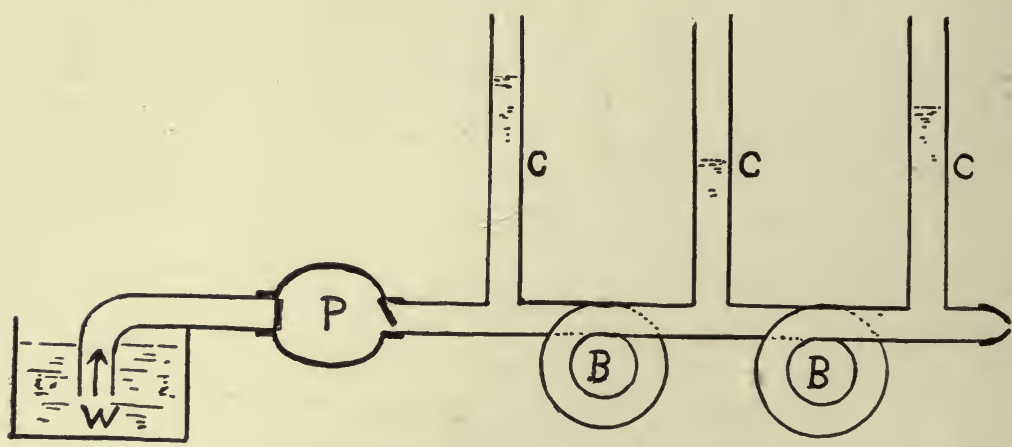

Fig. 65.-Schema Illustrating the Cause and Velocity of the Arterial Pulse.

$W$, Basin with water; $P$, valved rubber bulb; $B$, band-tubing between the laterals $C$.

Observe the changes in the caliber of the artery during inspiration and expiration. It increases during the former period, and decreases during the latter. Since the heart receives a larger quantity of blood during inspiration, it is capable of transferring a larger amount of it into the arteries. The pulmonary blood-vessels are at this time more widely dilated, and permit a free through flow.

Free the common carotid artery from its fascia and insert in it a glass cannula. Fill the latter with a solution of sodium carbonate. Fill the rubber tubing and central limb of the mercury manometer with the same solution. Determine the zero-line or line of atmospheric pressure $(760 \mathrm{~mm}$. Hg.). Connect the arterial cannula with the manometer.

Annotation.-The zero-line is ascertained as follows: Bring the level of the mercury in the central limb of the manometer on the same level with the carotid artery. Connect the rubber tubing with a glass bulb filled half-way with a solution of sodium carbonate. Adjust the level of this solution to the level of the mercury in the central limb of the manometer. At this time the recording needle of the float, 
resting upon the mercury in the distal limb of the manometer, is set at zero. In order to be able to retain this line during the succeeding experiments, adjust the sta-

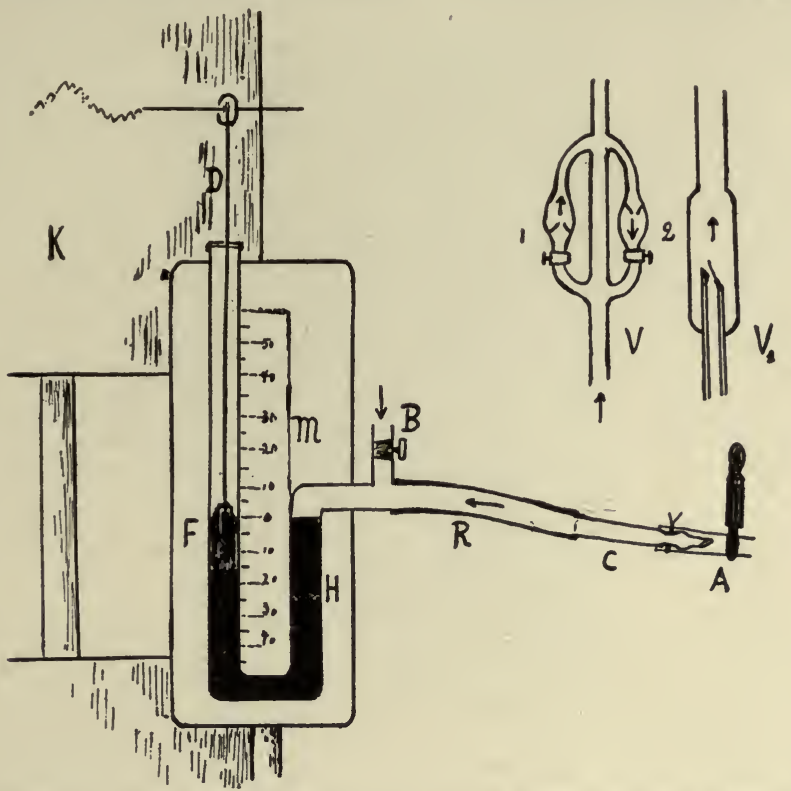

Fig. 66.-Schema to Show the Connection Made Between the Artery and ManOMETER.

$M$, Manometer; $H$, mercurial column; $F$, float; $D$, recording needle; $K$, kymograph; $B$, tube leading to reservoir filled with solution of sodium carbonate; $R$, rubber tubing filled with sodium carbonate solution; $C$, glass cannula in artery; $A$, clip upon artery; $V$, maximal-minimal valve (Frank) to be inserted in this circuit; 1 , maximal, 2, minimal side; $V_{1}$, maximal valve of Hurthle. A minimal valve is obtained by inverting the central tube.

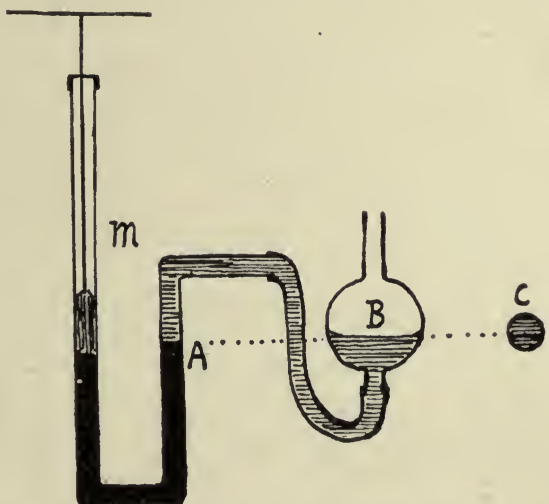

Fig. 67.-Diagram to Show the Adjustments Necessary for Determining the ZERo-line of the MANOMETER $(M)$.

Its central limb $(A)$ is brought upon the same horizontal line as the level of the water in the glass bulb $(B)$ when held at the level of the blood-vessel $(C)$.

tionary recording lever of the manometer to the level of the writing needle of the float. The latter may then rise above this zero-line to indicate the blood-pressure. 
The recording needle of the manometer having been placed against the smoked paper of the kymograph, allow a small quantity of sodium

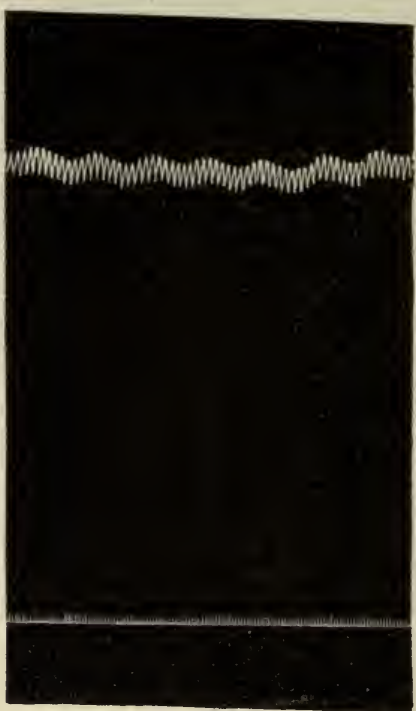

Fig. 68.-Record of Blood-Pressure Showing the Cardiac and Respiratory VariaTIONS.

The time, registered in seconds, serves as the abscissa.

carbonate to enter the connecting tubes from a receptacle suspended above the manometer. Remove the clip from the artery, and adjust

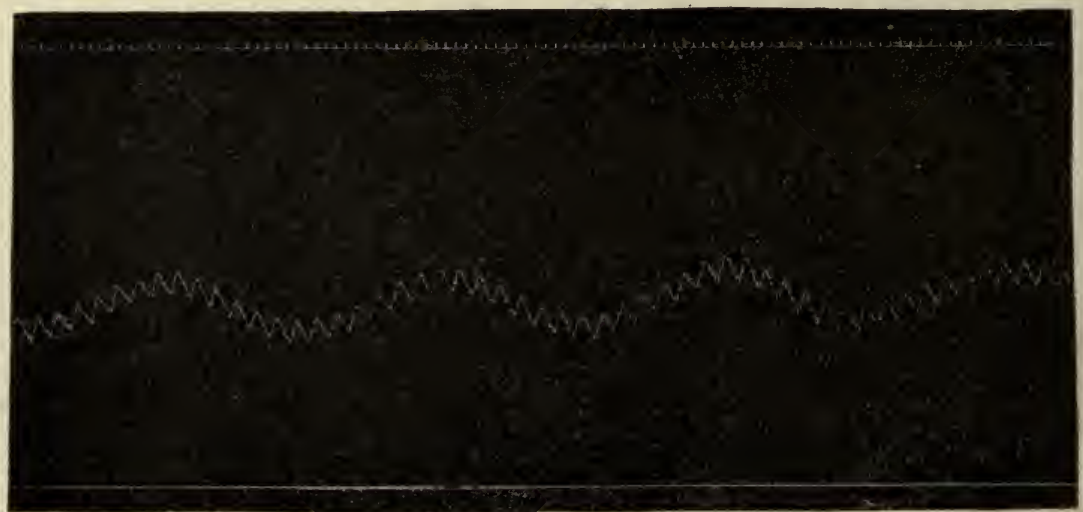

Fig. 69.-Traube-Hering Curves.

The time is given in seconds. The smallest pulsations represent the cardiac variations, those of intermediate size the respiratory variations, and the large waves the Traube-Hering variations.

the speed of the drum so as to render the individual cardiac variations clearly perceptible. Observe the character of the respiratory varia- 
tions in pressure and note that each is made up of a number of cardiac variations. Does the tracing show Traube-Hering curves, which are rhythmic waves including several respiratory variations? Ascertain the height of the pressure.

Annotation.- It is best to establish a certain degree of pressure in the manometer before the blood is actually allowed to act against the mercury. In a cat or dog we may expect to obtain a pressure equalling 120 to $130 \mathrm{~mm}$. Hg. Thus, if we establish a pressure of $100 \mathrm{~mm}$. Hg. beforehand, only a small quantity of blood need pass into the glass cannula to produce the additional pressure. On the other hand, if the recording needle is allowed to remain at zero to begin with, the aforesaid degree of pressure can only be produced by the passage of a considerable portion of the blood into the connecting tubes. In small animals this would give rise to a material decrease in pressure, and would greatly favor the occurrence of coagulation in the connecting tube.

To obtain the blood-pressure, measure the distance between the zero-line and the midline of the oscillations recorded by the manometer $(\mathrm{H})$. Employ the formula:

$$
\mathrm{P}=2 \mathrm{H}-\frac{\mathrm{H}}{13.5}
$$

Obviously, the recording needle registers merely the upward movement of the mercury in the distal limb of the manometer. It must be remembered, however, that the mercury in the central limb moves at this time an equal distance in a downward direction, and hence the distance between the zero-line and the oscillations in pressure, as registered by the needle, must be multiplied by 2 . In addition, the correction $\frac{\mathrm{H}}{13.5}$ must be made, because the blood acts against mercury, which is 13.5 times as heavy as water.

In order to obtain a more accurate mean pressure a large number of measurements must be made, first, of the systolic pressure, and secondly, of the diastolic pressure. The arithmetic mean between the averages of these pressures may be taken to be the mean blood-pressure. More accurate determinations require planometric measurements.

4. Influence of Posture.-Remove the clip from the artery, and again record the blood-pressure. Raise the posterior extremities of the animal vertically upward. Keep them in this position for ten seconds and again lower them slowly. By this means a large quantity of blood is forced into the head-circuit of the animal, occasioning a rise in the carotid pressure. Such increases in blood-pressure are usually associated with a reflex slowing of the heart.

5. Compression of the Abdominal Aorta.-The preceding result may also be obtained by temporarily occluding important subdivisions of the vascular system. Thus, if the thumb is pressed flat against the spinal column somewhat below the left costal arch of the animal, the resultant compresssion of the abdominal aorta must lead to an engorgement of the head-circuit and a corresponding rise in the carotid pressure. Observe that the sudden release of the compression usually causes the pressure to fall below normal, and that several seconds then commonly elapse before it again assumes its normal height. It is only natural to assume that the sudden inrush of the blood into the previously empty 
blood-vessels of the posterior part of the body must cause a temporary reduction in the quantity of the blood allotted to the head-circuit.

6. Influence of Hemorrhage.-Insert a cannula in the femoral artery. While a tracing of the carotid pressure is being recorded remove the clip from the femoral artery and allow 20 c.c. of blood to escape into a beaker. Observe the systolic increase in the force of the ejection. Again apply the clamp and allow the pressure to adjust itself. What factors are involved in this compensation? Again withdraw 20 c.c. of blood, and continue this procedure until the pressure has fallen to zero. Carefully observe the symptoms of excessive hemorrhage, as displayed by the changes in the character of the respiratory movements and the rate and force of the heart. How much blood has been withdrawn, and what proportion of the total quantity present in this animal? Kill the animal by giving an excessive amount of ether. 




\section{LESSON XX}

\section{THE CIRCULATION (Continued)}

VENOUS VALVES. INFLUENCE OF DYSPNEA UPON THE BLOOD-PRESSURE. ACTION OF AMYL NITRITE AND ADRENALIN. HEMORRHAGE

1. Position and Function of the Venous Valves.-Ask the subject to hold his arm in a dependent position. Encircle the forearm with a piece of rubber tubing, but not too tightly. Raise the arm. Note the distended condition of the veins upon the dorsal aspect of the hand. Select the point of confluency of two veins. With the tip of the index-finger of your left hand occlude one branch distally to this point. With the tip of the index-finger of your right hand brush along this vein in a direction from periphery to center, emptying the blood into the collecting vein. Observe that the vein so emptied does not fill again until you have removed the distal finger and have allowed a

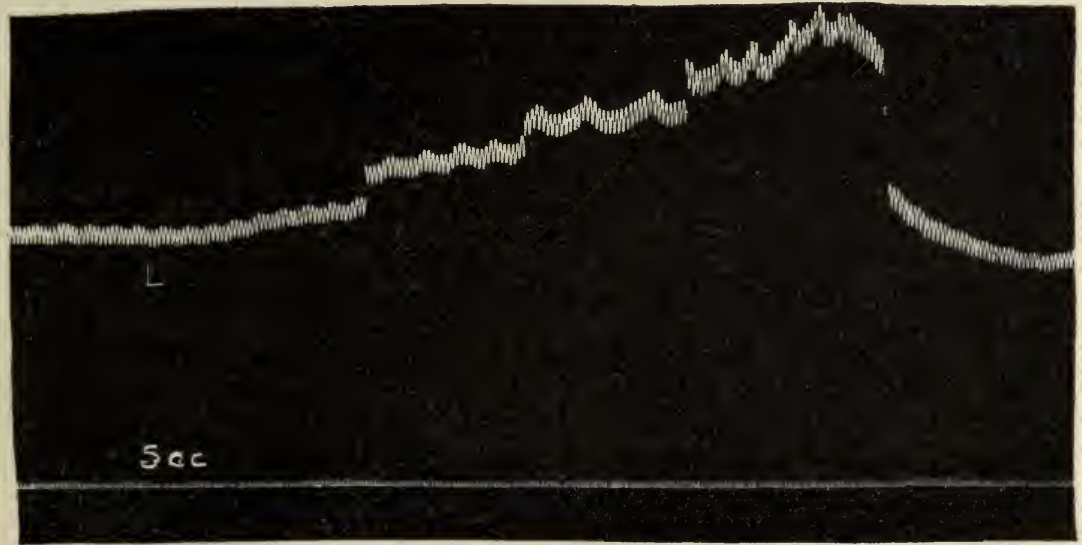

Fig. 70.-Record of the Carotid Blood-pressure During Dyspnea (Dog). At $L$ the tracheal tube was held shut until the blood-pressure began to drop.

certain quantity of blood to flow into it from its tributaries. Moreover, while emptied a marked prominence is developed at its point of confluency with the larger vein, indicating the position of the valve guarding its central orifice. This experiment was employed by Harvey to prove the circulation of the blood. Examine a preparation of a segment of vein preserved in alcohol.

2. Blood-pressure. Influence of Dyspnea.-Anesthetize a mammal and maintain the anesthesia until it has been killed. Perform tracheotomy. Insert a cannula in the carotid artery and connect it with the mercury manometer. Record the blood-pressure upon a slowly revolv- 
ing drum. Occlude the tracheal cannula with the tip of your finger. Observe that the respiratory movements gradually assume a labored character and that the respiratory variations in blood-pressure are thereby rendered more conspicuous. The blood-pressure rises gradually. The frequency of the heart, which is increased at first, is soon lessened. The heart then assumes a markedly diastolic character and causes the blood-pressure to fall. Immediately remove your finger from the tracheal cannula and wait until normal hemodynamic conditions have again been established. What factors are involved in the initial rise in blood-pressure?

3. Effect of Amyl Nitrite.-Place a glass bead filled with amyl nitrite in a small glass bottle. Break it between the tongs of an artery forceps. Place the palm of your hand over the mouth of the bottle. Record the blood-pressure upon a slowly revolving drum. Allow the animal to inhale the amyl nitrite by holding the end of the rubber tube attached to the tracheal cannula in the bottle. Remove the tube from the bottle as soon as the blood-pressure begins to fall. Naturally, the

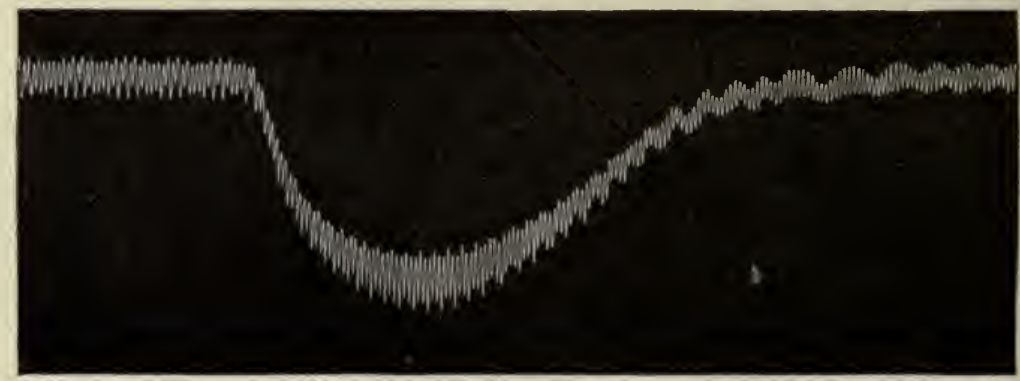

Fig. 71.-Effect of Amyl Nitrite on Blood-pressure (Dog).

pressure will continue to drop even after this time until the amyl nitrite, which has been absorbed from the inspiratory air, has been rendered inert. Subsequent to this point, however, it will rise slowly until normal conditions have again been established. Explain in detail the action of this agent.

What are the hemodynamic conditions established during attacks of angina pectoris? What changes may be effected during these attacks by inhalations of amyl nitrite?

Replace the clip upon the artery after each experiment. If coagulation has set in, flush out the tubing as well as the cannula with sodium carbonate solution.

4. Effect of Adrenalin.-Add 1 c.c. of adrenalin (solution $1: 1000$ ) to 10 c.c. of saline solution. Expose the right external jugular vein. Place a clip upon it centrally and insert a cannula distally to the clip (toward the heart). Fill the cannula with normal saline solution and connect it by means of a short piece of rubber tubing with a pipet containing the aforesaid solution of adrenalin. Fasten the pipet in a 
stand obliquely above the vein. Record the blood-pressure. Release the clip slightly and allow 1 to 2 c.c. of the solution of adrenalin to enter the venous circulation. Make a mark upon the paper, indicating the moment when the injection was made.

Determine the length of the latent period intervening between the injection and the rise in blood-pressure. Account for this interval. Note the character of the reaction and explain its cause.

What is the effect of adrenalin when applied to bleeding surfaces? State why adrenalin is added to solutions used for purposes of infusion after hemorrhage.

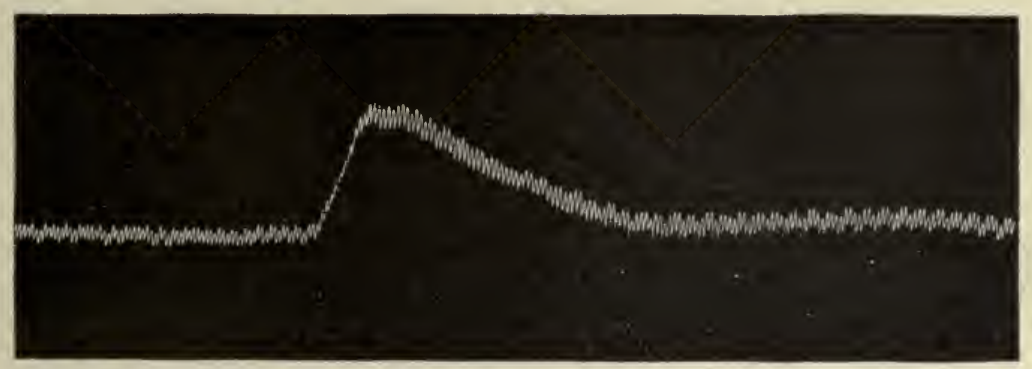

Fig. 72.-Effect of Adrenalin on Blood-pressure (Dog).

Annotation.-Since adrenalin deteriorates on standing, and especially when exposed to light, its strength can only be determined in a physiologic way. Consequently, adjust the dose to the reaction obtained, $i$. e., give it in smaller quantities if the rise is excessive and if the heart-beats assume a pronouncedly diastolic character.

5. Hemorrhage and Saline Injection.-Heat a considerable quantity of physiologic salt solution to $38^{\circ} \mathrm{C}$. Draw 100 c.c. of this solution into a pipet and connect the latter with the cannula in the jugular vein. Record the blood-pressure. Bleed the animal through the femoral artery until the carotid pressure has fallen to about $50 \mathrm{~mm}$. $\mathrm{Hg}$. Allow 50 c.c. of saline solution to enter the venous circulation. What effect is produced thereby? Repeat the injection until the pressure has become normal. How large an amount of the solution has been injected? Kill the animal by giving an excessive amount of ether. 



$$
\sqrt{3}
$$




\section{THE CIRCULATION (Continued)}

\section{THE EFFECT OF DIVISION AND STIMULATION OF THE VAGUS NERVE UPON THE BLOOD-PRESSURE AND ACTION OF THE HEART}

1. Stimulation of the Intact Vagi Nerves.-Etherize a mammal and continue the anesthesia until the animal has been killed at the end of the following experiments: Perform tracheotomy. Listen to the heart sounds and locate the cardiac impulse. With the tips of your indexfingers press gently upon the skin of the neck overlying the vagi nerves. Do you notice a reduction in the rate of the heart? Expose the common carotid artery on the left side and insert in it a straight cannula to be connected later on with the mercury manometer. Also expose the

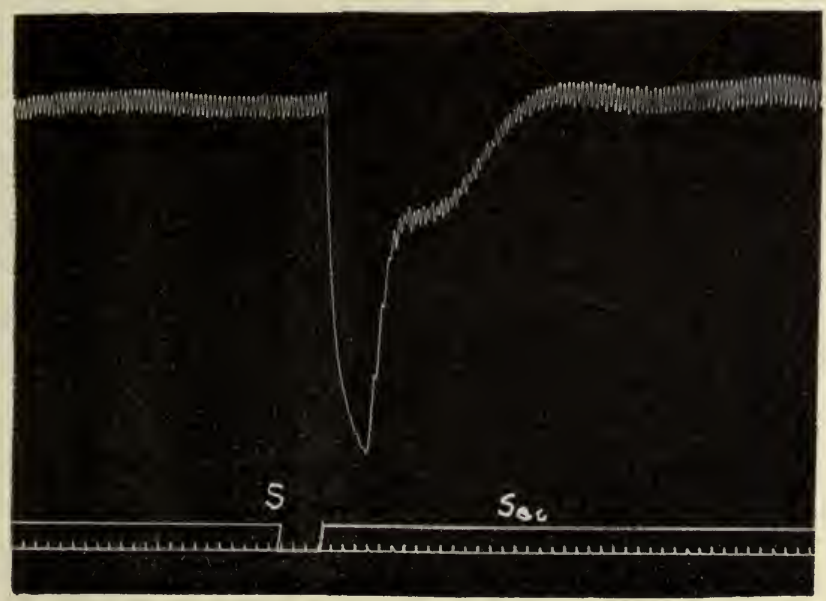

Fig. 73.-Record of Carotid Blood-pressure.

$S$, Stimulation of left vagus nerve. The fall in pressure is followed by compensatory changes before the normal pressure is again established.

common carotid artery on the right side, and separate both vagi nerves from the sheaths of these blood-vessels. Place each in a loose silk ligature. Arrange the stimulating apparatus to yield a quickly interrupted current of medium strength.

Apply the electrodes to the left intact vagus and stimulate briefly, while the normal blood-pressure is being recorded. In case the effect is too indefinite, increase the strength of the current slightly. Test the opposite nerve in the same manner and with the same strength of current. Do you notice a difference in the inhibitor power of these nerves? In each case allow the pressure to return to normal before you 
stimulate again. Note the compensatory variations occurring at this time.

2. Division of One Vagus Nerve.-Apply another loose ligature to each vagus nerve. Record the normal blood-pressure. Ligate one vagus twice and cut between the ligatures, so that its central and distal stumps may be stimulated separately. Indicate the moment of the division upon the tracing. What changes do you observe in the bloodpressure? Obtain the cardiac frequency from the tracing before and after the section. To what factor do you attribute the rise in pressure?

Stimulate the distal end of this nerve. After the pressure has returned to normal stimulate the central end of this nerve. What deductions may be drawn regarding the inhibitor qualities of the vagi?

Annotation.-It has been stated above that the inhibitor power of the vagi nerves differs in different animals. Thus, cardio-inhibition is easily obtained in the dog, but only with difficulty in cats. Secondly, in certain animals the stimulation of the central end of one vagus - the other being intact-produces a reflex slowing of the heart.

3. Division of Both Vagi Nerves.-Record the blood-pressure and divide the opposite vagus in the same manner. Determine the cardiac rate before and after the section. Are the effects previously noted now more pronounced? Stimulate the distal and central ends of this nerve. Compare the inhibitory power of the left nerve with that of the right.

Annotation.-The inhipitor power of these nerves varies considerably. Sometimes the left and sometimes the right nerve is the stronger of the two, but most generally the right. With both nerves divided, the stimulation of either central end must necessarily fail to produce a reflex slowing of the heart.

4. Inhibition of the Exposed Heart.-Test the artificial respiration apparatus and adjust the check-valve for an air current of medium volume. Connect the tracheal cannula of the animal with the etherbottle. Incise the skin over the sternum. By means of a pair of strong scissors cut through this bone directly in the median line, avoiding the mammary arteries. Institute artificial respiration. Vary the position of the stop-cock upon the inlet tube to give to the lungs a normal degree of inflation. Stop the bleeding by applying a compress or by torsion and ligation of the vessels. Pull the edges of the sternum apart by means of a strong string drawn around the board. Open the pericardial sac and inspect the heart, repeating the observations made in the course of Lesson XVI. Stimulate the distal end of either vagus nerve. Note that the heart stops in diastole, greatly distended by blood. Observe the swollen condition of the central veins, indicating a rise in venous pressure.

Endeavor to retain the heart in the inhibited condition by prolonging the duration of the stimulation. What happens? When the heart resumes its beat it is apparent that it contracts first in the region of the pace-maker, giving a well-marked contraction wave. 
5. Intracardiac Pressure.-Expose the right external jugular vein. Place a clip upon it centrally and ligate it about $2 \mathrm{~cm}$. farther distally. Open this vein between the clip and the ligature and insert the end of a long, hollow probe filled with physiologic salt solution. Place a ligature rather loosely around that portion of the vein which contains the end of the catheter. Connect the latter with a membrane manometer. Remove the clip from the vein and push the catheter slowly downward until its free end comes to lie in the right auricular cavity. Obtain a tracing of the right intra-auricular pressure.

Push the catheter through the auriculoventricular orifice into the right ventricle, taking care not to injure the tricuspid valve. Obtain a tracing of the right intraventricular pressure. Withdraw the catheter and replace the clip upon the vein.

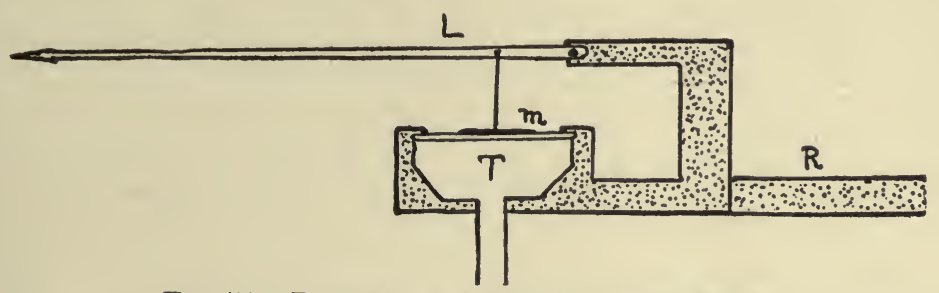

Fig. 74.-Diagram of Membrane Manometer.

$M$, Rubber membrane connected with writing lever $(L)$. The drum $(T)$ is connected with the cannula in the blood-vessel; $R, \operatorname{rod}$ to fasten manometer to stand.

Study the character of each tracing and compare them with one another. Calculate the pressures so obtained by comparing the oscillations of the membrane with those of a mercury manometer.

6. Entrance of Air Into the Circulation.-Insert a glass cannula in the right external jugular vein. Record the blood-pressure, and apply a stethoscope to the chest wall. Force a small amount of air into the aforesaid vein. After a short latent period the blood-pressure will begin to fall, this fall being accompanied by a peculiar noise produced by the frothing of the blood within the heart, as the valve flaps beat against the bubbles of air.

Kill the animal by an overdose of ether. Open the heart and examine the character of the blood. Dissect the heart, repeating the observations made in the course of Lesson XV. 





\section{LESSON XXII}

\section{THE CIRCULATION (Continued) \\ THE VASOMOTOR ACTION OF THE CERVICAL SYMPATHETIC, DEPRESSOR, AND SCIATIC NERVES}

1. The Cervical Sympathetic Nerve.-Etherize a rabbit and maintain the anesthesia throughout the following experiments: Perform tracheotomy. Make an incision along the inner border of the sternocleidomastoid muscle, and retract this muscle laterally outward. Do not disturb the relationship of these parts by dissection. Identify the carotid artery and the large vagus nerve right neighboring. In addition, identify two delicate nerves, one white and one gray in color. The former are the depressor fibers of the vagus which pursue a separate course in the rabbit, and the latter, the sympathetic fibers uniting the inferior and superior cervical ganglia of this system. Place each in a loose silk ligature. Shift the head of the animal so that the ear on the side operated on may be placed in a position for inspection. Hold an incandescent lamp behind it. Identify the central artery of the ear and the lateral venous collecting channels. Arrange the electric apparatus for stimulation with a quickly interrupted current of very moderate intensity. Gently raise the cervical sympathetic nerve from the wound and place it in small shielded electrodes. Stimulate for a period of about fifteen to twenty seconds. Note that after an appreciable latent period the central artery becomes less conspicuous and

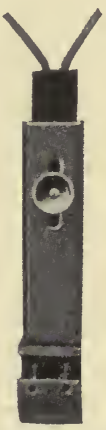

Fig. 75.-SHIELDED

Electrodes.

(Harvard A pparatus Co.) eventually disappears altogether. Discontinue the stimulation and allow circulatory conditions to become normal again. What conclusions may be drawn from this experiment regarding the vasomotor action of this nerve?

Raise the cervical sympathetic nerve and cut it between two ligatures. Inspect the blood-vessels of this ear. Note that its vascularity is now much greater than before and exceeds that of the opposite organ. It also possesses a higher temperature. Obviously, the division of this nerve has given rise to a relaxation of the blood-vessels of the corresponding ear. Stimulate the distal end of the divided nerve and repeat the observations made previously(Fig. 76).

It might be well to allude at this time to the influence of this nerve upon the size of the pupil, although the student cannot be expected as yet to be familiar with this particular reaction. Since the cervical 
sympathetic nerve contains certain fibers which innervate the radial muscle cells of the iris, its excitation must give rise to an increase in the size of the pupil. Consequently, if any doubt exists as to the character of the nerve isolated, this reaction may be employed to make sure that it is the sympathetic.
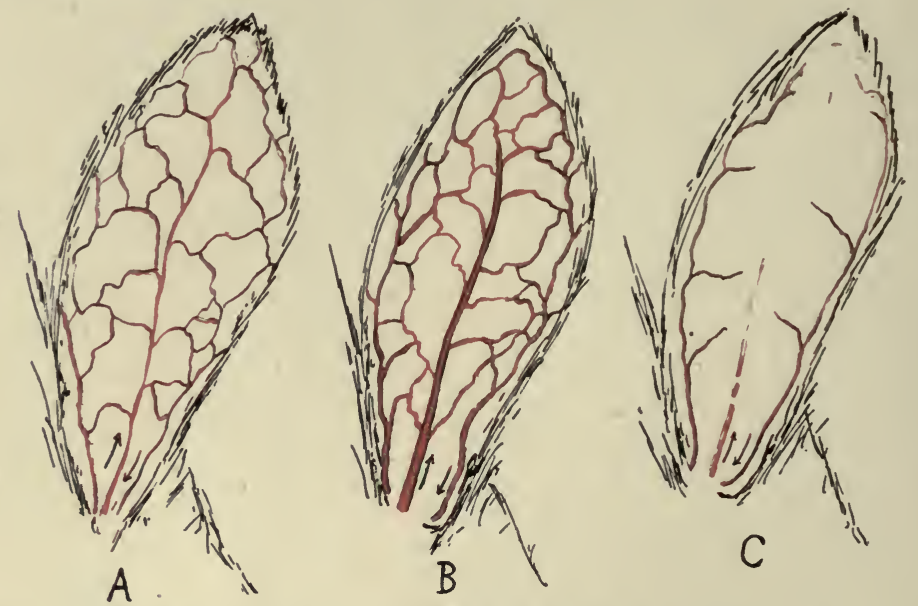

Fig. 76.-The Vasomotor Reaction in the Ear of the Rabbit on Division and Stimulation of the Cervical Sympathetic Nerve.

$A$, Normal $B$, After division of the cervical sympathetic nerve. $C$, On stimulation of the distal end of the divided cervical sympathetic nerve.

2. The Depressor Nerve.-Insert a straight eannula in the left common carotid artery and connect it with the mercury manometer. Place the depressor nerve in shielded electrodes, and arrange the electric apparatus for stimulation with a quickly interrupted current of mod-

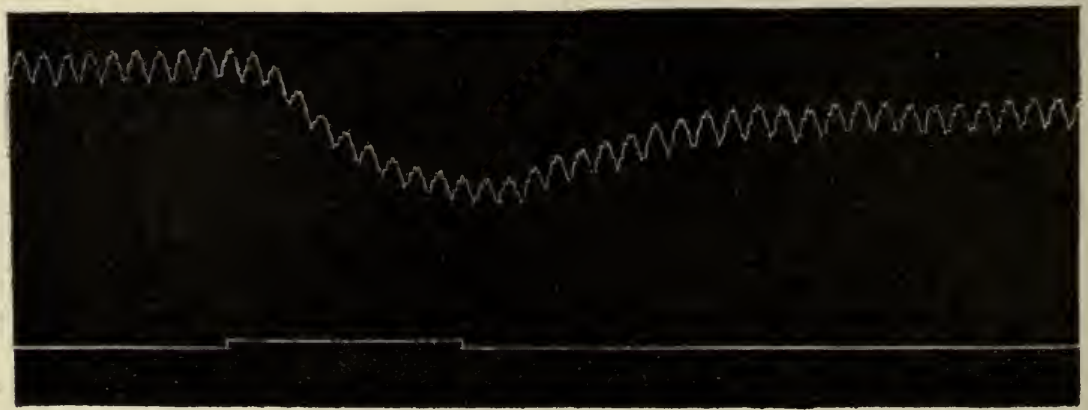

Fig. 77.-Record of the Carotid Blood-pressure in Rabit During Stimulation of THE Depressor Nerve.

erate strength. Record the blood-pressure and stimulate the aforesaid nerve for about twenty seconds. Note the fall in blood-pressure and also the decrease in the frequency of the heart. Allow the pressure to return to normal. 
Apply two ligatures to this nerve, a few millimeters apart, and cut between them. Record the blood-pressure and stimulate its central and distal (heart) ends successively at intervals. Since the excitation of the distal end remains ineffective, what conclusions may be drawn regarding the direction of conduction in this nerve? What two factors should be held responsible for the fall in blood-pressure?

Divide both vagi nerves and repeat the stimulation of the central end of the depressor as soon as the changes ordinarily following the section of the aforesaid nerves, have become thoroughly established. Observe that henceforth the fall in blood-pressure is no longer associated with a reduction in the frequency of the heart. Give a detailed explanation of the function of the depressor nerve, and mention conditions during which this mechanism is called into play.

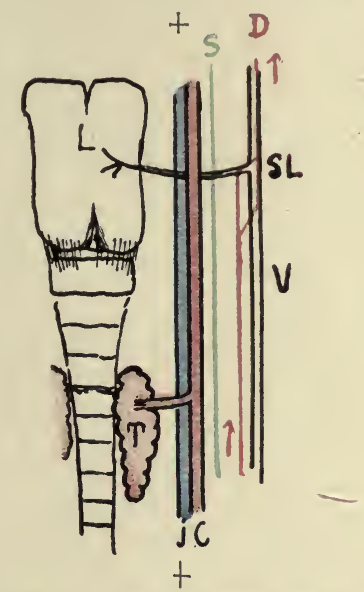

Fig. 78.-Diagram to Show the Course of the Depressor Nerve in the Rabiti.

$L$, Larynx; $T$, thyroid gland; $j$, int. jugular vein; $C$, carotid artery; $S$, sympathetic nerve extending between the superior and inferior cervical ganglia; $V$, vagus nerve; $S L$, sup. laryngeal nerve; $D$, depressor nerve, entering the vagus by two branches. The vagus is pulled over, permitting the sympathetic to appear next to the carotid artery.

3. The Sciatic Nerve.-Test the artificial respiration apparatus and connect the ether-bottle with the tracheal cannula. Prepare a solution of curare. Inject 1 c.c. of this solution intravenously by means of a syringe. Allow this agent to produce its characteristic effect, and institute artificial respiration as soon as the diaphragm shows the first indications of ceasing its action (fifteen minutes). Expose the sciatic nerve in the thigh; place a ligature upon it, and cut it distally to the ligature. Record the carotid pressure and stimulate the central end of this nerve with a quickly interrupted current of moderate strength. As a rule, currents of medium strength and frequency give rise to a reflex vasoconstriction, and hence to an increase in the carotid bloodpressure. Since the motor plates of the skeletal musculature have been paralyzed by the curare, this result cannot be referred to the mechanical influence of contracting muscle tissue. 
Weaken the strength of the current considerably by moving the secondary coil farther away from the primary. Stimulate the nerve in the same manner with single induction shocks repeated at intervals of about half a second. This procedure usually gives rise to a reflex vasodilatation, and hence to a fall in the carotid blood-pressure. Consequently, the sciatic nerve contains vasoconstrictor as well as vasodilator fibers. Kill the animal by an overdose of ether.

Annotation.-In isolating the sciatic nerve make use of the following landmarks: Seek a point midway between the ischium and great trochanter of the femur, and draw an imaginary line from here to the anterior aspect of the knee-joint. Direct the incision along this line about the middle of the thigh and separate the adjoining muscles until the fatty tissue investing the sciatic nerve has been exposed. 




\section{LESSON XXIII}

\section{THE CIRCULATION (Continued)}

\section{THE VASOMOTOR ACTION OF THE GREATER SPLANCHNIC NERVE. THE VASCULARITY OF THE KIDNEY. ONCOMETRY}

1. The Greater Splanchnic Nerve.-Anesthetize a cat and maintain deep anesthesia throughout the following experiments: Perform tracheotomy. Insert a straight cannula in the left common carotid artery and arrange the apparatus for recording the blood-pressure. Make a median incision through the skin of the abdomen and incise the linea alba. Introduce the middle and index-fingers of your left hand through this opening and by using them as a guide enlarge the incision upward as far as the ensiform cartilage, and downward-as far as the region of

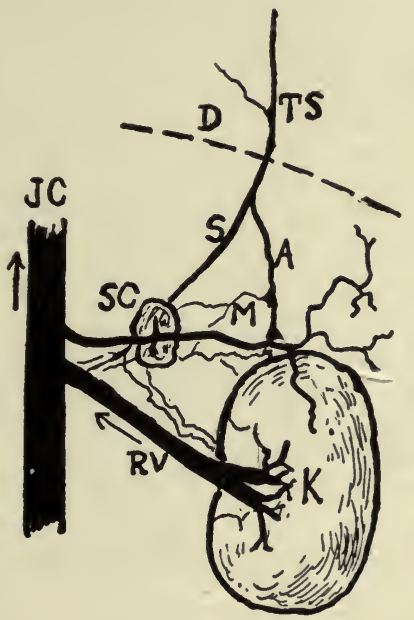

Fig. 79.-Region of Left Kidnex.

$J C$, Inferior vena cava; $R V$, renal vein; $K$, kidney; $S C$, left adrenal body with corresponding vein; $S$, greater splanchnic nerve; $A$, abdominal sympathetic nerve; $M$, minor splanchnic nerves, from lumbar ganglia to suprarenal plexus below adrenal body; $D$, diaphragm; $T S$, thoracic sympathetic nerve.

the bladder. Apply a cloth moistened with warmed saline solution to the abdomen.

Arrange the electric apparatus for stimulation with a quickly interrupted current of medium strength. Procure a pair of small shielded electrodes and connect them with the secondary coil of the inductorium. Ask the assistant to expose the region above the left kidney, holding this organ and the liver away from the field of operation. Identify the left suprarenal body at the junction of the left suprarenal vein with the inferior vena cava. It appears as a rounded, pea-shaped mass, possessing a pink color. With two forceps split the peritoneum and fascia 
along its right (as you look at it) upper margin. Very carefully search for a small nerve in the fatty tissue of this region pursuing an oblique course upward through the crus of the diaphragm. This is the left greater splanchnic nerve, connecting the thoracic sympathetic system with the solar plexus. Place a loose silk ligature around it and secure it in shielded electrodes. Insulate by means of a narrow piece of rubber membrane and dry cotton. Allow the parts to close in around the electrodes and apply a warm cloth to the abdomen.

Allow the carotid blood-pressure to be recorded. Stimulate the aforesaid nerve for about ten seconds until you have obtained an appre-

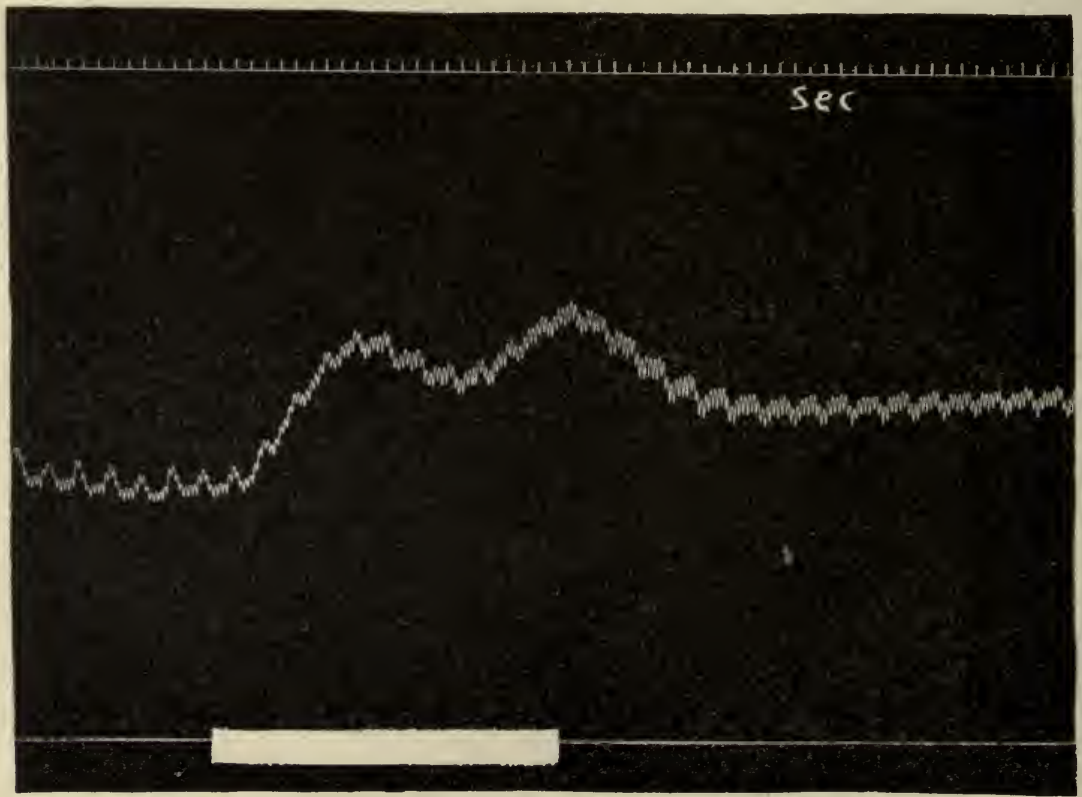

Fig. 80.-Splanchnic Rise in Blood-Pressure.

ciable rise in pressure. Repeat this procedure after an interval. Apply the clip to the artery. Study the character of the tracing so obtained.

Annotation.-The rise in blood-pressure usually consists of two phases. The first is brought about by the vasoconstriction, resulting in the so-called splanchnic organs in consequence of the excitation of this nerve. This rise appears within a few seconds after the onset of the stimulation and finds its origin in a rapid transfer of blood from the abdominal organs into the channels of the general circulatory system. The second rise appears about ten seconds after the first, and is due to the constriction of the blood-vessels of the general system by the adrenin liberated in consequence of the excitation of this nerve. Obviously, only the left adrenal body is involved, because the innervation of these organs by the splanchnic nerve is inilateral.

Record the blood-pressure. Quickly tighten the silk ligature so as to crush this nerve. Observe the gradual fall in pressure which is 
brought about by the fact that the abdominal blond-vessels innervated by this nerve, lose their tonus and relax. Consequently, a certain quantity of blood will now find its way from the general circulation into the relaxed blood-vessels of the splanchnic organs.

Apply the shielded electrodes to the distal (abdominal) end of the divided nerve. Replace the abdominal organs and cover the abdomen with a warm, moist cloth. Record the blood-pressure and stimulate the splanchnic nerve as described above. Owing to the lower general pressure, the rise will now be more pronounced. Disconnect the manometer and ligate the carotid artery.

2. The Vasomotor Supply of the Kidney.-Procure an oncometer large enough to contain the kidney of this animal. Fill the outer compartments of this instrument with warm water until their membranous inner walls have become moderately distended. Separate the left kidney from the fatty tissue surrounding it, but do not injure its capsule. Place the lower hemispheric part of the oncometer underneath this organ. Adjust its cover so that the, kidney assumes a central position between the rubber membranes, taking special care that its blood-vessels as well as the ureter pursue a perfectly normal course through the slit-like lateral orifice of the oncometer. Connect the compartment in the cover of this instrument by means of a piece of rubber tubing with a membrane tambour or piston recorder. It is not essential that this connecting tube be filled with water. Cover the abdomen with a warm, moist cloth.

Register the excursions of the recording lever upon the smoked paper of a slowly revolving kymograph. Observe that the volume of the kidney undergoes cardiac and respiratory variations, $i$. e., it is increased during each systolic phase of the heart and suffers a more general increase during the entire inspiratory period.

Temporarily obstruct both femoral arteries. Note the effect upon the volume of the kidney. Explain. Temporarily occlude the carotid arteries. Observe the change in the volume of the kidney. Explain.

Adjust the shielded electrodes to the distal end of the divided left greater splanchnic nerve. While registering the volume curve of the corresponding kidney stimulate this nerve with a quickly interrupted current of moderate strength. Account for the decided reduction in its volume. Kill the animal by an excessive amount of ether.

Dissect the region of the left kidney and adrenal body. Identify the organs on the opposite side, and also the suprarenal, mesenteric, and celiac ganglia and plexuses of the solar sympathetic system. Trace the greater splanchnic nerve into the thorax, identifying the thoracic sympathetic nerve and stellate ganglion. Enucleate the adrenal bodies. Bisect them and identify their cortical and medullary portions.

3. Vasomotor Phenomena in Man.-Fill a large beaker with icewater and another with water heated to $40^{\circ} \mathrm{C}$. Introduce your hands for a short time. Withdraw, and note the effect upon the color and condition of the skin. Explain. 





\section{LESSON XXIV}

\section{THE CIRCULATION (Continued)}

\section{THE INDIRECT METHOD OF MEASURING BLOOD-PRESSURE. EFFECT OF POSTURE AND EXERCISE}

1. The Application of the Sphygmomanometer.-Place the indexfinger of your left hand upon the skin over the brachial artery of the . subject. Palpate the radial pulse with the index-and middle fingers of your right hand. Gently compress the brachial artery until the radial pulse can no longer be felt.

Place the index-finger of your left hand upon the skin over the brachial artery. With your right hand adjust the chest-piece of a

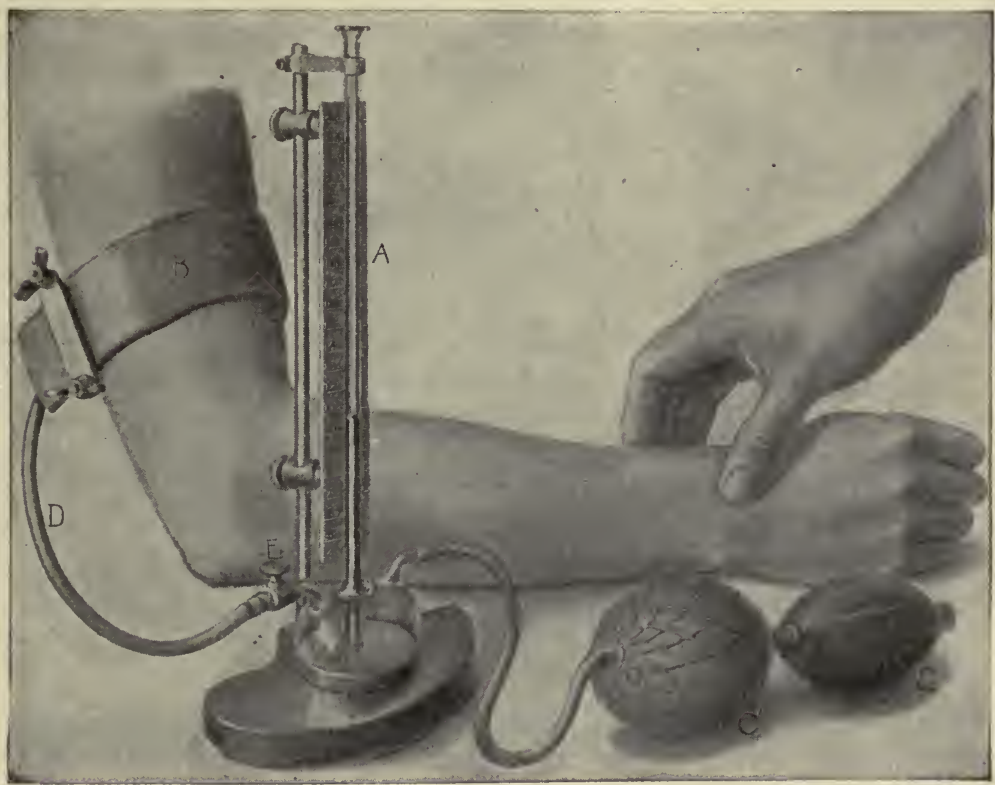

Fig. 81.-Riva-Rocci's Sphygmomanometer, Early Type. (From Janeway's "Clinical Study of Blood-pressure.")

stethoscope upon the flexor surface of the elbow-joint. Do you hear a sound? Gently compress the brachial artery. Describe the character of the sound now heard. Explain its cause.

The sphygmomanometer most commonly used in making these tests consists of $(a)$ a mercury manometer, $(b)$ a narrow rubber cuff to fit around the arm, and $(c)$ an inflating bulb with exhaust valve. In the sphygmotonometer the place of the mercurial indicator is taken by a steel 
spring which must be calibrated from time to time by comparing its movements with those of a column of mercury under equal degrees of pressure. Since the mercury manometer usually consists of one vertical limb leading away from a central reservoir, the pressure may be read off directly without correction. Some instruments, however, contain a $\mathrm{U}$-shaped tube, in which case the pressure, as read off from the ascending limb, must be multiplied by 2 .

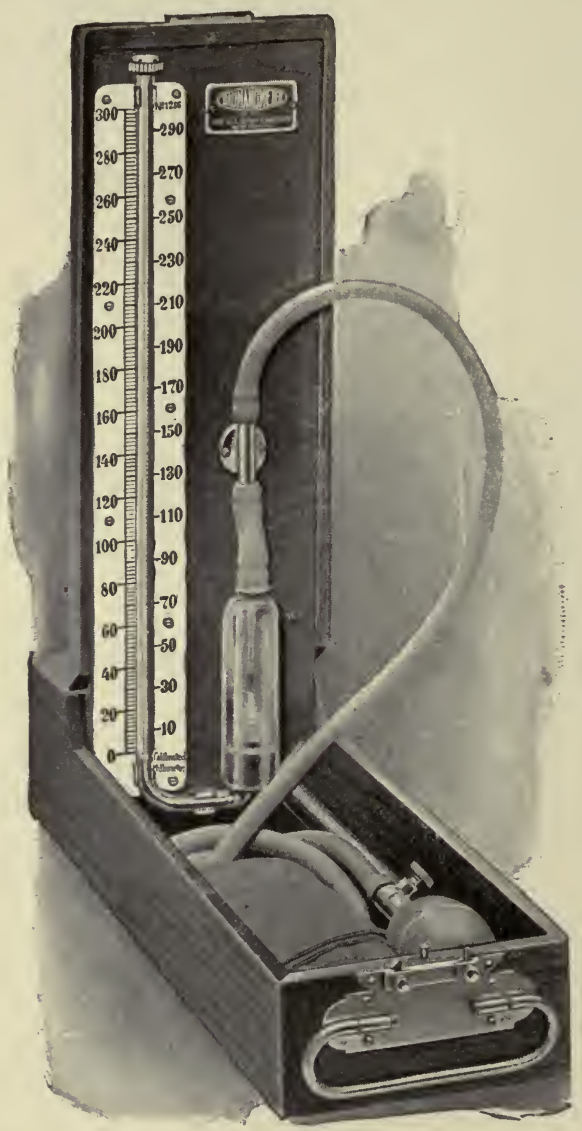

Fig. 82.-Sphygmomanometer, Recent Type. (Manufactured by W. A. Baum Co., N. Y.)

The subject should be comfortably seated in a chair with his right forearm resting upon a table. Adjust the armpiece of the sphygmomanometer (modification of the Riva-Rocci instrument) to the arm of the subject. Be sure that the lower edge of the cuff does not encroach upon the elbow and is not applied too loosely. Two methods may be followed in determining the blood-pressure, namely, palpation and auscultation.

(a) Palpation.-Having adjusted the armpiece accurately, locate the 
radial pulse with the fingers of the right hand. Close the exhaust valve and compress the rubber bulb rapidly to raise the pressure to about $140 \mathrm{~mm} . \mathrm{Hg}$. In a young person possessing an elastic vascular system and in the sitting position, we would not expect to find a pressure higher than this. Consequently, the pressure in the cuff overcomes at this time the internal pressure. The brachial artery is fully compressed and the radial pulse obliterated. Now gradually deflate until the radial pulse just barely makes itself felt. Read the pressure and deflate rapidly. Be sure to compress the arm for only the shortest possible time. Repeat again after intervals until you have acquired this technic thoroughly and are able to obtain correct results. This gives the systolic blood-pressure.

(b) Auscultation.-Place the chest-piece of a stethoscope over the region of the bifurcation of the brachial artery directly below the lower edge of the cuff. Inflate rapidly as before, then deflate gradually. No sound is heard when the brachial artery is obstructed. At the very moment, however, when the arterial pressure just overcomes the outside pressure a sound is produced indicative of the systolic gushes of blood through the constriction. Read the pressure, which, as has just been stated, is the systolic. Practice this method a number of times. Compare these results with those obtained previously by palpation.

The estimation of the diastolic pressure may be attempted during the process of palpation by carefully noting the amplitude of the oscillations of the mercury of the manometer. When the systolic pressure breaks through the constriction these fluctuations are small, but become much larger as the diastolic value is approached. Below this point their amplitude again decreases. A better way is to ascertain this value by the method of auscultation. Having determined the moment when the systolic sound just appears, continue to deflate slowly. The sound becomes louder; soon reaches a maximum, and then suddenly disappears. Read the pressure at this point. It indicates the diastolic pressure.

Deduct the value of the diastolic pressure from that of the systolic. This gives the pulse-pressure, which varies under ordinary conditions between 35 and $40 \mathrm{~mm}$. $\mathrm{Hg}$.

2. Effect of Posture.-Determine the systolic and diastolic pressures of the subject while resting horizontally upon the table. with his head upon a pillow. Repeat this test after he has assumed the sitting position, and again after he has assumed the standing position. Tabulate the results and determine the pulse-pressure. Explain the results. What bearing do they possess upon the condition of the vascular system?

Crampton has attempted to obtain an approximate estimate of the condition or vascular tone of a person by balancing the increase in the heart rate with the increase or decrease in blood-pressure resulting on standing up. The range of the systolic pressure has been found to be between +10 and $-10 \mathrm{~mm} . \mathrm{Hg}$, and the increase in the frequency of the heart between 0 and 44. By assigning equal percentages to these 
the following scale has been constructed, in accordance with which the vascular tone may be expressed in percentages:

Heart rate,
increase.

Thus, a person who on standing up shows an increase in the cardiac rate of 10 beats, and an increase in the blood-pressure of $10 \mathrm{~mm} . \mathrm{Hg}$, would be in the condition A (95 per cent.).

Barach calculates the energy-index in accordance with the following example:

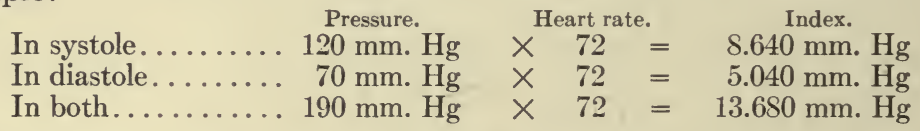

The highest energy-index in a still normal person has been found to lie close to $20,000 \mathrm{~mm}$. $\mathrm{Hg}$ in a minute, and the lowest somewhere about 10,000 .

3. The Effect of Exercise.-Determine the normal systolic bloodpressure and rate of the heart with the subject standing. Repeat these determinations immediately after the subject has made forty flexions and extensions of the arms or thirty knee bendings in one minute. Repeat one, two, three, four, and five minutes afterward. Construct a curve to show the course of the pressure and cardiac frequency.

Determine the cardiac frequency and blood-pressure in a subject before and after he has made a stationary run lasting one-half minute.

Determine the cardiac frequency and blood-pressure in a subject before and after he has ascended forty steps in the course of one minute.

4. Venous Pressure.-Hold the hand against the chest in the region of the heart. Note the degree of filling of the veins. Raise the hand slowly until the veins collapse. Determine the distance between this level and the level of the heart. It corresponds to the height of the column of blood supported by the heart. 



\section{LESSON XXV}

\section{THE CIRCULATION (Concluded)}

\section{THE CHARACTER AND VELOCITY OF THE ARTERIAL AND VENOUS PULSATIONS. POLYGRAPHY}

1. The Application of the Sphygmograph.-Determine the rate of the subject's heart by palpation of the radial pulse. How is the rate affected by the act of swallowing?

Study the construction of the sphygmograph. It usually consists of a vibrating rod which acts in magnified form upon a recording lever. The end of the rod is equipped with an oval projection which is adjusted over the artery. Apply this instrument securely to the radial artery and record a number of normal sphygmographic curves. Study their character.

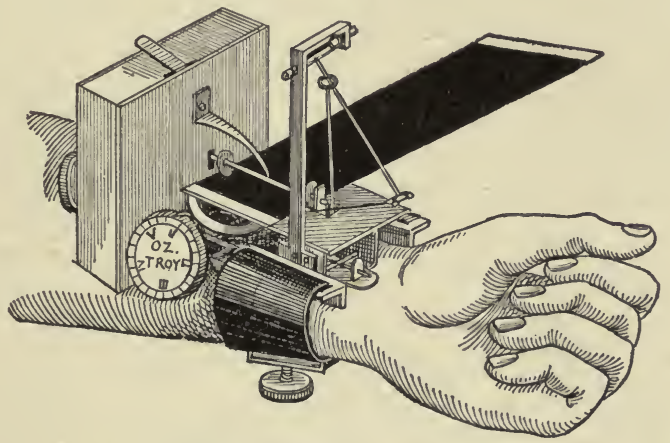

Fig. 83.-The Dudgeon Sphygmograph in Position. (Howell.)

Ask the subject to close his mouth and nostrils with the fingers of the free hand and exhale forcibly. Explain the result (Valsalva's experiment).

2. Relation Between the Arterial Pulse and the Action of the Heart. -Obtain a sphygmographic record of the radial pulse in proper relation with a record of the apex-beat registered by means of a cardiograph. Note whether any extrasystoles are present.

Allow a cardiograph and a sphygmograph (radial artery) to register their excursions in the same ordinate upon a rapidly rotating drum, above the record of a tuning-fork. Obtain the approximate distance between the ascending aorta and the radial artery, and ascertain the speed of the pulse-wave by computing the difference of the cardiac and radial impulses.

3. The Use of the Phlebograph.-Apply a metal „or glass cup to the jugular fossa and connect it with a recording tambour. Also register 
the time. Study the character of the phlebogram, and compare it with the curve of intra-auricular pressure. Under what conditions does the phlebogram become of special value? What changes would tricuspid regurgitation produce in the character of the tracing?

Obtain a phlebogram in proper relation with a sphygmogram of the radial artery, or with a cardiogram. When would a record of this kind prove of special diagnostic value?

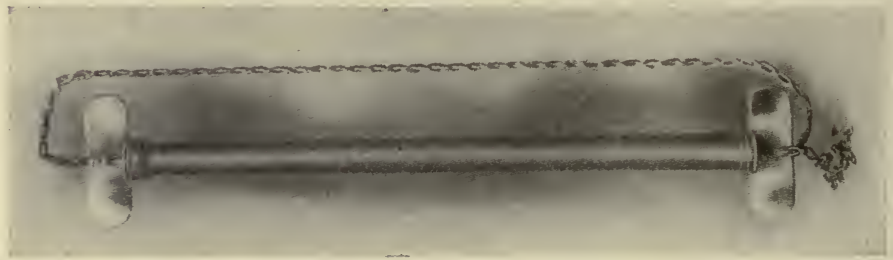

Fig. 84.-Pneumograph. (Harvard Apparatus Co.)

Annotation.-As the name indicates, a jugular cup consists of a hemispheric capsule of metal or glass which is placed flat against the region of the central end of the external jugular vein. The pulsations of this blood-vessel are transmitted to a recording drum.

4. Relation Between the Cardiac and Respiratory Activities.-Adjust a stethograph upon the chest of the subject and connect it with a recording tambour. Place a receiving cup over the region of the carotid artery and connect it with a recording drum arranged to register in the

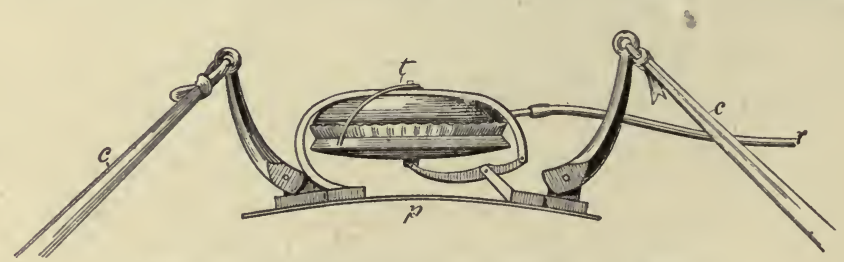

Fig. 85.-Marey's Pneumograph. (Verdin.)

The instrument consists of a tambour $(t)$, mounted on a flexible metal plate $(p)$. By means of the bands $c$ and $c$ the metal plate is tied to the chest. Any increase or decrease in the size of the chest will then affect the tambour by the lever arrangement shown in the figure. These changes in the tambour are transmitted through the tube $r$ as pressure changes in the contained air to a second tambour (not shown in the figure) which records them upon a smoked drum. (Howell.)

same vertical line as the former. Beneath these writing levers adjust the marker of a Jaquet chronograph. While the subject assumes a perfectly inattentive attitude, record a number of respiratory cycles upon a drum revolving at a moderate speed. How many cardiac cycles does each respiratory cycle embrace? Do you observe an increase in the cardiac frequency on inspiration? Explain this variation.

Annotation.-A simple stethograph may be made by fastening a small rubber pouch by means of a broad linen strap against the chest. The single orifice of this pouch is connected by means of a cannula and a piece of rubber tubing with a record- 
ing tambour. Another convenient form consists of a long rubber tube about 2 $\mathrm{cm}$. in diameter and closed at one end. Its other end is connected with a recording tambour by means of small tubing (Fig. S4). 'The interior of the large tube is occupied by a long spiral spring. When applied transversely around the chest this tube will be lengthened on inspiration and air will be drawn into it, causing the membrane of the recording tambour to be displaced inward. On expiration, the recoil of the tube, aided by the spiral, increases the pressure within its lumen and forces the rubber membrane of the tambour outward. Marey's stethograph (Fig. 85) consists of a metal plate which is fastened to the surface of the chest by means of a strap. 'The changes in the tension of this plate suffered by it in consequence of the respiratory movements, are communicated by means of a lever to a receiving drum. The latter, in turn, communicates with a recording drum. 




\section{LESSON XXVI \\ RESPIRATION \\ MECHANICS OF RESPIRATION}

1. The Spirometer.-Study the construction of the spirometer (Hutchinson). Breathe normally, then close the nostrils and exhale normally through the mouthpiece of this instrument. Determine the movement of the pointer and calculate the volume of the tidal air.

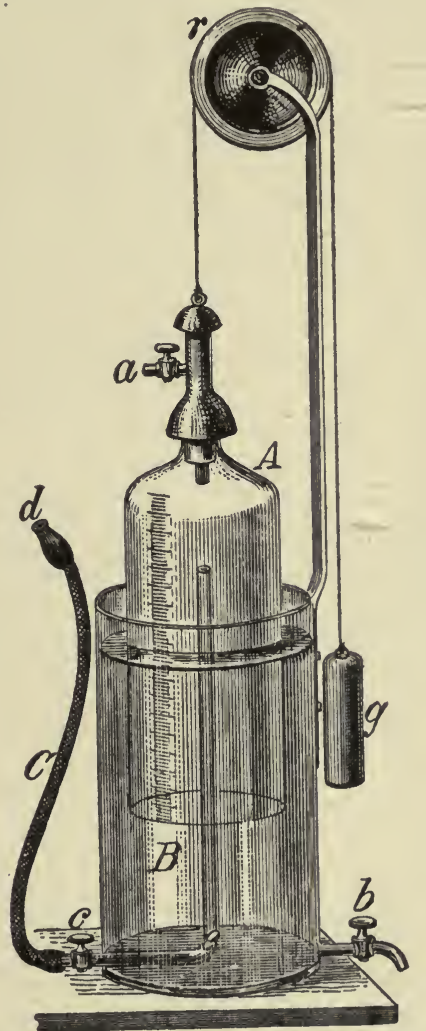

Fig. 86.-Wintrich's Modification of Hutchinson's Spirometer. (Reichert.)

Breathe normally; then exhale forcibly through the mouthpiece. Determine the volume of the supplemental air.

Set the spirometer at 5000 c.c. Breathe normally; then inspire deeply through the mouthpiece. Determine the volume of the complemental air. 
Set the spirometer at zero. After several normal respirations, inspire as deeply as possible and immediately expire as much as possible through the mouthpiece of the spirometer. Ascertain the vital capacity.

2. Schema Illustrating the Action of the Thorax.-Examine the construction of the model represented in Fig. 87.

(a) Normal Respiration.-Lower and raise the rubber membrane closing the bell-jar. Observe that the rubber pouch representing the lung is expanded during the first movement, and decreased in size during the second. Clearly, these changes are brought about by an action upon the entire external surface of the rubber pouch. When the pressure in the "intrapleural space" (between the surface of the rubber pouch and the wall of the bell-jar) is decreased by the downward move-

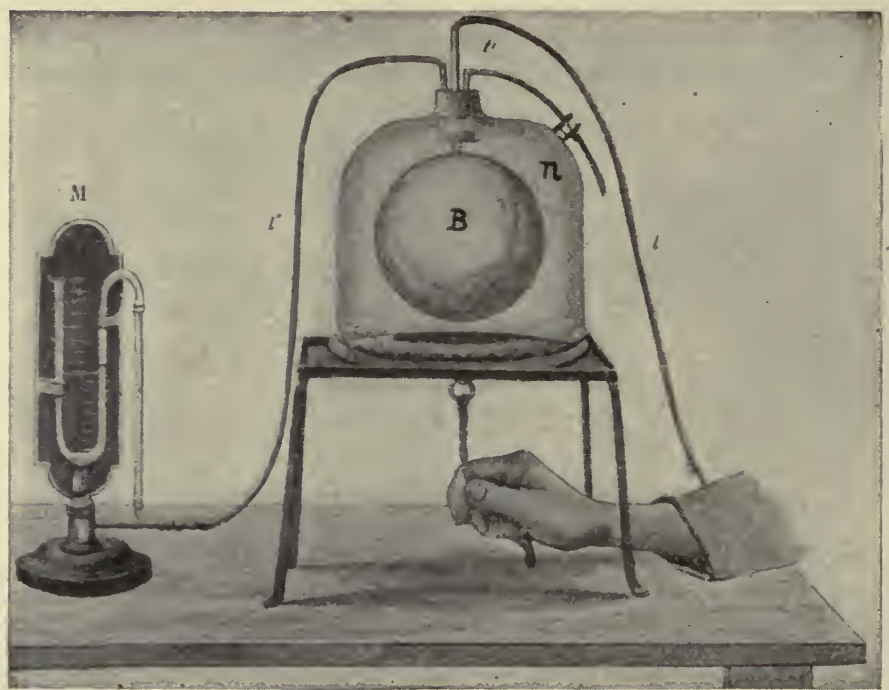

Fig. 87.-Apparatus Illustrating the Expansion of the Lung. (Laulanie.) $n$, Bell jar; $B$, lung in form of rubber balloon; $M$, manometer in connection with "intrapleural space." Another manometer may be connected with the inlet-tube to register the "intrapulmonic pressure."

ment of the "diaphragm" (rubber membrane), the walls of the "lung" (rubber pouch) are pulled uniformly outward. An area of low pressure is thereby established within the "lungs," forcing a certain quantity of air to flow through the "trachea" (inlet tube) into the pouch. The reverse relationships are established by the upward movement of the "diaphragm," simulating expiration.

The two manometers fastened to the top of this model are connected, on the one hand, with the "intrapleural space" and, on the other, with the "trachea." Carefully observe the variations in the pressures during inspiration and expiration, showing that the fall in "intrapleural pressure" is responsible for the expansion of the "lung" and the subsequent influx of air into its spaces. Normally, of course, the intrapleural 
space is a capillary space filled with pleural fluid. This implies that the surface of the lung is everywhere in close contact with the inner surface of the wall of the thorax.

(b) Forced Respiration.-Lower and raise the "diaphragm" more forcibly, thereby producing a more ample expansion of the "lung" and more decisive variations in pressure.

(c) Dyspnea and Asphyxia.-The former condition may be reproduced in a mechanical way by partially closing the stop-cock with which the inlet tube is equipped. This would correspond to an incomplete closure of the trachea and would greatly impair the interchange of the respiratory air. Note the resistance now acting against the "diaphragm." Obviously, this diminution in the caliber of the inlet tube must augment the variations in the intrapleural and intrapulmonic pressures.

Close the stop-cock completely, simulating the condition of asphyxia, or complete absence of air.

(d) Collapse of the Lungs.-Expand the "lung" by moving the "diaphragm" downward. Suddenly permit air to rush into the "intrapleural" space by slightly tilting the rubber cork closing the upper orifice of the bell-jar. Observe the immediate loss of "intrapleural" pressure and collapse of the "lung." Having in this way destroyed the "intrapleural" pressure, endeavor to expand the "lung" by moving the "diaphragm" downward.

3. Application of Above Principles to the Thorax of the Mammal.Anesthetize a cat and maintain the anesthesia throughout the following experiments: Perform tracheotomy. Make a median incision through the skin in the midventral line of the body, beginning near the tip of the sternum and extending well along the linea alba. Reflect the skin on each side so as to expose the ventral aspect of the thorax. Study the mechanism of normal respiration. Note the excursions of the diaphragm and contraction of the neighboring intercostal muscles. Observe the downward movement of the liver and stomach on inspiration. Which part of the chest is affected most in quiet breathing? Produce forced respiration by partially closing the rubber tube attached to the tracheal cannula. The normally diaphragmatic type of respiration is now gradually augmented by costal respiration. Observe also that the accessory movements of respiration are now much more conspicuous.

4. Action of the Diaphragm.-Open the abdominal cavity by an incision through the linea alba. Retract the margins of the wound and depress the liver. Observe: (a) The muscular and tendinous portions of the diaphragm, $(b)$ the general shape of this septum, $(c)$ the course of the fibers composing its muscular part, $(d)$ the manner of attachment and insertion of these fibers, and $(e)$ the character of their contraction.

Determine the distance traversed by the tendinous portion of the diaphragm on quiet inspiration. Identify the complemental space, bounded by the upper surface of the diaphragm and the opposite wall 
of the chest. Observe that this space is widened on inspiration and that the lower borders of the lungs are then drawn into it. Transilluminate this region, so that individual alveoli may be made out. Note that the tissue of the lung is in absolute contact with the tendinous portion of the diaphragm.

5. Intrapleural Pressure.-Procure a water manometer. Color the water with a little indigo-carmin. Connect the central tube of this manometer with a metal cannula, about $10 \mathrm{~cm}$. in length and curved at its end. Force the end of this cannula through the soft tissues of the seventh intercostal space and immediately turn it so that it comes to lie flat between the surface of the lung and the chest wall. Do not move it excessively, because this might give rise to a leakage of air into the intrapleural space and a collapse of the lung.

Observe that the liquid in the manometer is drawn toward the chest as soon as the end of the cannula has perforated the wall of the thorax and has forced the lung tissue away from the inner surface of the chest wall. The end of the cannula thus comes to lie between the visceral and parietal layers of -the pleura. The air in this artificial cavity, and hence also the liquid in the manometer, is exposed to the elastic recoil of the lung tissue. The force of this recoil is indicated by the inward movement of the liquid $\left(\mathrm{cm} . \mathrm{H}_{2} \mathrm{O}\right)$. Inasmuch as the lung tissue is more highly stretched during the inspiratory period, this pressure must fluctuate. It approaches zero (line of atmospheric pressure, $760 \mathrm{~mm} . \mathrm{Hg}$ ) at the end of expiration and assumes a value of as much as $-5 \mathrm{~mm}$. $\mathrm{Hg}$ (756 to $755 \mathrm{~mm}$. $\mathrm{Hg}$ ) on quiet inspiration.

Partially occlude the rubber tube attached to the tracheal cannula. As the breathing assumes a labored character, these differences in the intrapleural pressure become more apparent $(-8$ to $-10 \mathrm{~mm}$. $\mathrm{Hg}$, in cats), because the lung tissue is now put under a greater elastic tension than during normal respiration.

6. Collapse of the Lung.-Inspect the tendinous portion of the diaphragm through the wound in the abdominal wall. Note that the pink pulmonary tissue lies in absolute contact with it. Twist the cannula slightly, allowing air to enter the pleural sac. The lung tissue will then be seen to recoil from the diaphragm (pneumothorax). This procedure most generally leads to the collapse of only one lung, while the other lung remains expansible and is capable of effecting an adequate interchange of the gases. Discuss the effect of placing the chest and posterior part of the animal in a compartment in which a negative pressure may be produced equaling the intrapleural.

Open the cavity of the thorax by a cut through the median line of the sternum. Institute artificial respiration through the ether bottle. Note the position of the cannula in the now actual and greatly enlarged intrapleural space. Withdraw it.

Temporarily discontinue the artificial respiration. Observe that the respiratory muscles continue to contract in spite of the fact that the lungs can no longer be expanded. As the $\mathrm{CO}_{2}$ accumulates in the 
system, stimulating the respiratory center to increased activity, these reflex movements frequently assume a spasmodic character.

Inspect the internal aspect of the chest wall, and note the shape of the thoracic cavity: Identify the pulmonary blood-vessels. Transilluminate the border of either lung, noting its alveolar structure. Identify portions of the visceral pleura below the apex of the heart. Note its structural peculiarities.

7. The Phrenic Nerves.-Isolate both phrenic nerves above the diaphragm and place them in loose ligatures. These nerves are easily found, because they descend in close proximity to the heart, the right selecting the highway of the inferior vena cava. Stimulate them separately with single induction shocks of moderate strength. Observe the resultant contraction of the corresponding half of the diaphragm and note the effect of this contraction upon the position of the abdominal organs. Kill the animal by an overdose of ether.

Trace the course of each phrenic nerve to its origin in the cervical portion of the spinal cord.

8. Swim Test.-Grasp the tracheal cannula with your left hand, and separate the trachea and lungs, together with the heart and large blood-vessels, from the neighboring parts. Place this mass in water, observing that the buoyancy of the lungs is sufficient to carry considerable additional weight. How would an atelectatic lung behave under these circumstances? What use is made of these facts medicolegally?

9. The Excised Lung.- Remove the metal cannula from the model, illustrating the manner in which the lungs are expanded. Insert this cannula in the trachea of the excised lungs, and suspend this preparation in the bell-jar. Repeat the observations made previously with the rubber pouch.

Remove the lungs. Compress them in the palm of your hand. Are you able thereby to expel all the air from them? Repeat the swim test. Explain your inability to render them air free. 





\section{LESSON XXVII}

\section{RESPIRATION (Continued)}

STETHOGRAPHY. METHODS OF ARTIFICIAL RESPIRATION. PULMOTOR.

1. Frequency of Respiration.-Study the movements of the thorax and abdomen. Differentiate between diaphragmatic and costal breathing. Measure the circumference of the chest at the level of the nipples on deep expiration and inspiration. Count the number of respirations of the subject first in the horizontal and then in the erect position. Count again after a stationary run lasting thirty seconds, and again after forty to fifty flexions and extensions of the arms or knee bendings. Explain the result.

2. Percussion and Auscultation.-Familiarize yourself with the normal percussion sound of the lungs as obtained, say, in the region below the clavicle. Outline the upper boundary of the liver and the area of cardiac dulness. Make a diagram showing the position of the thoracic viscera.

Familiarize yourself with the normal vesicular sounds of the lungs. Auscultate over the bronchi and trachea.

3. Stethography.-Adjust a stethograph to the chest of the subject while he is quietly sitting beside a table (see Fig. 84). Allow a recording drum to register the respiratory movements over the record of a Jaquet chronograph. Ask the subject to take two or three deep breaths in quick succession. Explain the result.

Record normal curves of respiration for about twenty seconds. Read aloud during the next forty seconds. Carefully note the modifications produced by the voice.

Ask the subject to hold his breath after a moderate inspiration. The breaking point will be reached in about forty seconds. Repeat after a deep inspiration. The breaking point will now be reached after about fifty seconds. Repeat after having breathed forcibly for two or three minutes. The breaking point then occurs after two or three minutes. Since the imperative demand to respire is due principally to the accumulation of carbon dioxid, and only in a lesser degree to the scarcity of oxygen, the breaking point cannot be much prolonged by the previous inhalation of oxygen.

Register a normal curve of respiration for about twenty seconds. Drink half a glass of water without stopping. Explain this modification.

Record normal curves of respiration. Ask the subject to approximate the tips of his two index-fingers. Explain the change in the character of the respiratory movements.

Take tracings of the modified respiratory curve of laughing, sneezing, and coughing. 
4. Influence of Carbon Dioxid.-Compress the nostrils of the subject and allow him to breathe from and into a rubber bag containing about 30 liters of air. As soon as the $\mathrm{CO}_{2}$ has accumulated sufficiently the subject will breathe more rapidly and more deeply until his rate is about forty to the minute. Stop the experiment soon after the occurrence of the hyperpnea.

Repeat this experiment after having interposed a receptacle of 1 or 2 liters capacity containing soda-lime or sticks of sodium hydrate. The carbon dioxid will be absorbed, while the oxygen in the bag is being used up. Consequently, there will be no hyperpnea. Only a few minutes will be required to establish this fact. Immediately allow the subject to breathe normal air, otherwise the oxygen deficiency will lead to cyanosis, frontal headache, and unconsciousness.

5. Artificial Respiration.-Familiarize yourself with the Sylvester and Schäfer methods of artificial respiration. Place the subject upon his back with the feet somewhat elevated. Take a position at the head

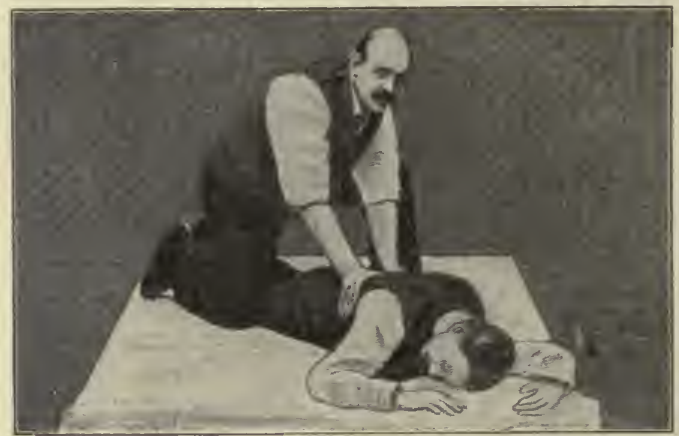

Fig. 88.-Position to Be Adopted for Effecting Artificial Respiration in Cases of Drowning. (Schaefer.)

of the subject and grasp his wrists. Now bring the forearms against the sides of the chest and press gently inward and downward against the ribs. Release the pressure, allowing the elasticity of the chest wall to restore normal conditions. Bring the arms above the head so as to stretch the accessory muscles and to enlarge the chest still further. Repeat this procedure at the rate of eighteen times in a minute (Sylvester).

Place the subject upon his ventral surface with a roller cushion under the epigastric region. Take a position over the legs of the subject, facing his head. Place the palms of your hands against the posterior and lateral aspect of the subject's lower ribs. Bring an even and gentle pressure to bear upon this region. Release the pressure so as to allow. the elasticity of the chest wall to restore normal conditions. Repeat this procedure at the rate of sixteen to eighteen times in a minute (Schäfer). What are the advantages of this method?

Study the construction and action of the pulmotor. What are its advantages and disadvantages? Contrast. 


\section{LESSON XXVIII \\ RESPIRATION (Continued) \\ NERVOUS REGULATION OF RESPIRATION}

1. Accessory Movements of Respiration.-Anesthetize a mammal and continue the anesthesia throughout the following experiments: Perform tracheotomy. Observe the movements of the facial muscles and note especially the changes in the size of the nostrils during inspiration and expiration. Render these movements more conspicuous by temporarily occluding the rubber tube attached to the tracheal cannula.

2. Self-regulation of Respiration.-Attach a pair of bellows to the tracheal cannula. Suddenly inflate the lungs. Note the expiratory effort immediately ensuing. Suddenly deflate the lungs and observe that the animal makes an immediate effort at inspiration. Explain these results upon the basis of the self-regulatory function of the vagi nerves.

3. The Trigeminal and Glossopharyngeal Nerves.-Apply a stethograph to the chest of this animal and register the respiratory movements upon the paper of a slowly revolving kymograph. Stimulate the nasal lining by means of a small plug of cotton attached to the end of a stick of wood. Note the resultant inhibition of respiration and forced expiratory efforts (act of sneezing). Enumerate the different nervous parts involved in this reflex. Stimulate the lining of the fauces and pharynx in the same manner, producing thereby those modifications of respiration which constitute the act of coughing. Trace the course of this reflex.

4. The Larynx.-Make a median incision through the skin covering the-region of the larynx and hyoid bone. Ligate the vein crossing the larynx and reflect the skin. Identify the thyroid and cricoid cartilages. Note the movability of the larynx and trachea. Isolate the superior and inferior laryngeal branches of the vagus on both sides, and place them in loose silk ligatures.

Annotation.-The superior laryngeal nerve pursues a course transversely across from the vagus nerve, and enters the lateral aspect of the thyroid cartilage. It is the largest nerve of this region. The inferior laryngeal nerve is isolated most readily below the larynx. It pursues a course upward along the trachea to enter the inferior aspect of the larynx.

Cut across the pharynx between the hyoid bone and upper margin of the thyroid cartilage. Bring the tip of the epiglottis through the incision and secure it by means of a pair of artery forceps. Enlarge the incision laterally so that the larynx may be raised and an unobstructed view be obtained of its interior. Identify the true and false vocal cords, the glottis, and the ventricles. Observe the alterations in the size and shape of the glottis in quiet inspiration and expiration. 
Temporarily occlude the rubber tube attached to the tracheal cannula, producing dyspnea and thereby rendering these variations more conspicuous.

Stimulate the lining of the larynx with a tuft of cotton fastened to a wooden stick. Note the resultant inhibition of respiration and the forced efforts at expiration (act of coughing). Compare these effects

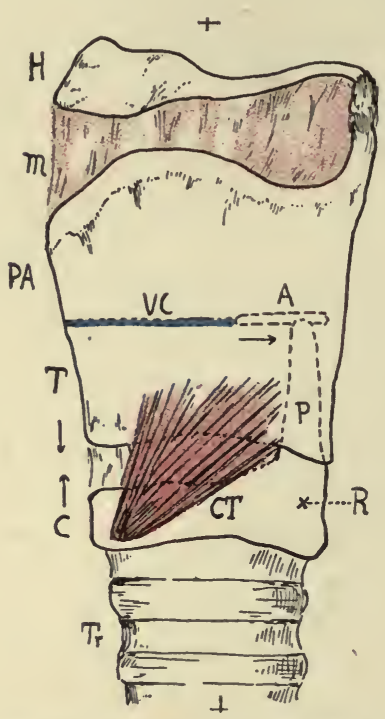

Fig. 89.-LATERAL View OF LARYNX to Illustrate the ACtion of the CricoTHYROID MUSCLE.

$H$, Hyoid bone; $M$, thyrohyoid membranes; $P A$, pomum Adami; $T$, thyroid cartilage; $C$, cricoid cartilage; $T r$, trachea; $C T$, cricothyroid muscle; $P$, vertical plate of cricoid with $(A)$ arytenoid cartilages placed transversely upon its articulating processes; $V C$, vocal cords; $R$, imaginary center of rotation of cricoid. When cricothyroid muscle contracts, $T$ and $C$ are brought closer together, while $A$ is forced away from $P A$.

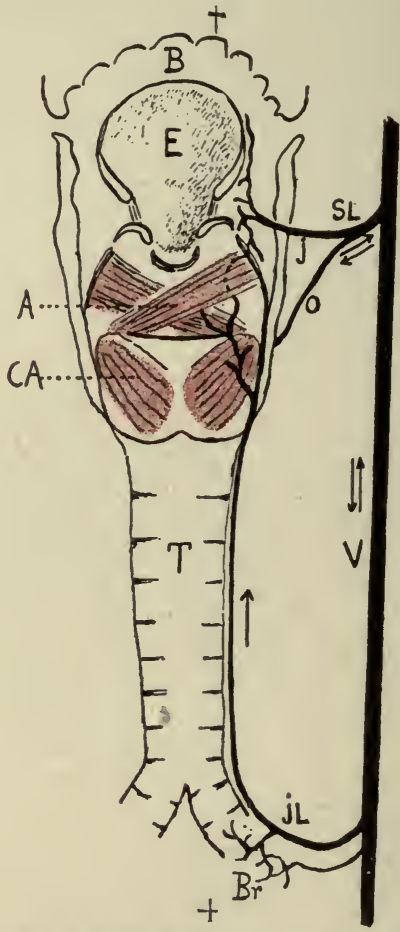

Fig. 90. - The Innervation of the Larynx (Posterior View; ONe Side).

$B$, Base of tongue; $E$, epiglottis; $A$, arytenoid muscles; $C A$, crico-arytenoid muscle; $T$, trachea; $V$, vagus nerve; $S L$, superior laryngeal nerve; $J$ and $O$, its inner and outer branches; $J L$, inferior laryngeal nerve; $B r$, vagal fibers innervating bronchial musculature.

with those commonly observed after the entrance of a foreign body into the larynx.

5. The Superior Laryngeal Nerve.-Place the intact superior laryngeal nerve in shielded electrodes and stimulate very briefly. Analyze the effect produced thereby upon $(a)$ the general character of the respiratory movements, and (b) the action of the laryngeal muscles.

Divide the superior laryngeal nerve between two ligatures. Stimulate its distal as well as its central end repeatedly, analyzing the effects produced in each case. 
Annotation.-The superior laryngeal nerve is chiefly a sensory nerve, but also embraces a certain number of motor fibers which innervate the cricothyroid muscle. Consequently, the stimulation of the intact nerve must give rise to an inhibition of respiration and forced expiratory efforts, and secondly, to an approximation of the cricoid and thyroid cartilages and a greater tension of the vocal cords.

6. The Inferior Laryngeal Nerve.-Place the intact inferior laryngeal nerve in shielded electrodes. Stimulate briefly and note the effects of the stimulation upon $(a)$ the general character of the respiratory movements, and $(b)$ the action of the laryngeal muscles.

Divide the inferior laryngeal nerve between two ligatures. Stimulate its distal as well as its central end, and study the effects (if any) produced in each case.
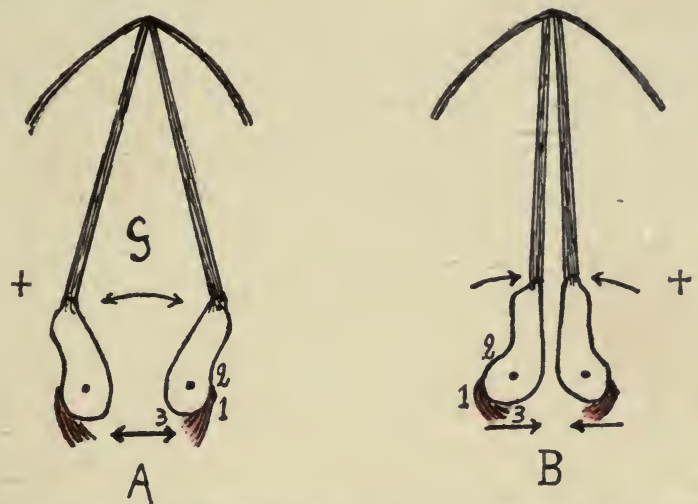

Fig. 91.-Diagram Illustrating the Abduction and Adduction of the Vocal Cords.

$A$, Abduction: 1, point of insertion of the post. crico-arytenoid muscle; $G$, glottis. $B$, adduction: 2, points of insertion of the lat. crico-arytenoid and thyro-arytenoid muscles; 3 , point of insertion of the arytenoid muscles. The dot indicates the position of the center of rotation of the arytenoid cartilages.

Annotation.-The inferior laryngeal nerve is a motor nerve, innervating the muscles of the larynx, except the cricothyroid. Consequently, its stimulation must give rise to peripheral effects only. These consist chiefly in contractions of the arytenoid muscles which have to do with the approximation of the vocal cords and the size of the glottis. In the dog the innervation of this nerve, as well as that of the superior laryngeal nerve, is unilateral.

7. The Main Trunk of the Vagus Nerve.-Expose both vagi nerves below their superior laryngeal branches. Divide each nerve between two ligatures. Observe that the rate of the respiratory movements is now greatly reduced, whereas their depth is increased. The total gas interchange is not seriously impaired. Stimulate the central end of either nerve with weak, medium, and strong currents. Discuss the part which the vagi nerves play in regulating the frequency and amplitude of the respiratory movements, formulating a concise picture of the self-regulation of respiration by means of these nerves. Administer an overdose of ether to the animal. 





\section{LESSON XXIX}

\section{RESPIRATION (Continued)}

\section{LOCALIZATION OF THE RESPIRATORY CENTER. PLACENTA. RESPIRA-}

TION IN THE FISH

1. The Localization of the Respiratory Center.-Anesthetize a cat and maintain the anesthesia until the animal has been killed. Perform tracheotomy. Place the animal upon its ventral surface. Make a median incision through the skin covering the region of the lower cervical and upper thoracic vertebræ. Identify the spinous process of the seventh cervical vertebra and follow this projection to the laminæ, retracting the spinal muscles. Stop bleeding by applying dry tampons or by torsion and ligation of the blood-vessels. Cut away the laminæ and process of the seventh vertebra until a short segment of the spinal cord has been exposed. With a curved needle draw a loose ligature around the cord.

Apply a stethograph to the chest of the animal and allow the recording drum to register the respiratory movements upon a slowly revolving kymograph. A sufficiently long normal record having been obtained, raise the spinal cord and divide it. The animal continues to respire normally, showing thereby that this lesion does not interfere with the efferent impulses to the muscles of respiration. Divide the students into three groups, and proceed as follows:

Group A.-Expose the spinal cord in the region of the third cervical vertebra. Place a loose ligature around it, and divide it after a short normal tracing has been taken. The respiratory movements cease almost immediately, owing to the fact that the transection has separated the nuclei of the respiratory nerves, principally those of the phrenic nerves, from the respiratory center. The latter, therefore, must lie above this section.

Group B.-Make a median incision through the skin covering the occiput. Advance in the direction of the foramen magnum. Enlarge the opening until a clear view of the region of the pons is obtained. Divide the latter structure transversely above the medulla oblongata. Since the animal continues to breathe, the principal center of respiration must be situated below this level.

Group C.-Make a median incision through the skin covering the occiput. Palpate this region until a depression in the vertebral column is clearly felt through which it is possible to reach the medulla directly. Pierce this structure with a pointed instrument. Respiration ceases immediately.

Compile the results of the three groups of students and draw conclusions regarding the location of the respiratory center. 
2. The Placenta.-Procure a placenta. Wear rubber gloves or handle this organ only with instruments. Study its appearance and draw a diagram to illustrate its position in the uterus. Divide the umbilical cord and identify its constituent blood-vessels. Observe the character of the outer and inner surfaces of the placenta. Note that the umbilical vessels complete their subdivision before the substance of the placenta is reached. Follow a cluster of vessels and separate the tissue supplied by them from the tissue supplied by neighboring groups of vessels. Suspend the fringed tissue so isolated in a beaker filled with water. By this means one may reproduce the conditions normally existing in this organ, the water representing the maternal blood with which the fetal blood is in diffusion relation.

3. The Respiratory Movements in Fishes.-Carefully study the opening and closing of the gill plates. What is the relation between this movement and the movements of the floor of the mouth? Draw diagrams to show the parts involved in these movements. Show the position of the maxillary and bronchostegal valves during inspiration and expiration. 




\section{RESPIRATION (Continued)}

\section{THE CIRCULATION IN THE LUNG OF THE FROG. PHENOMENA OF INFLAMMATION. EFFECT OF CHANGES IN INTRATHORACIC PRESSURE UPON THE LESSER CIRCUIT}

1. The Lung of the Frog.-Pith a frog. Open its jaws widely, and draw a small curved needle and silk thread through the soft tissues around the orifice of the trachea. Insert the end of a straight glass cannula in the tracheal orifice and secure it by means of the ligature. Attach a short piece of rubber tubing to the cannula. . Open the abdominal cavity of the frog widely. Blow air gently through the cannula until both lungs have been fully inflated. Kink the rubber tube and apply a clip. Remove both lungs with the heart and suspend them until thoroughly dried.

Transilluminate them. Note the large central cavity in each as well as the individual alveolar spaces along the wall. Cut each lung in half and inspect its interior.

2. The Capillary Circulation in the Frog's Lung.-Pith a frog without losing any blood. Block the opening by means of a pointed piece of match. Proceed as has been described in paragraph 1, imparting to the lungs a moderate degree of inflation. Raise one lung out of its cavity and place it upon the glass fitted in the orifice of a plate of cork. such as has been described upon page 99 .

Place a cover-slip upon the upper surface of this lung and apply gentle pressure to flatten the latter. Study the blood flow under the low and high powers of a microscope.

Examine a preparation of injected pulmonary capillaries under the microscope.

3. Phenomena of Inflammation.-Allow a drop of a dilute solution of mustard to be drawn by capillarity under the glass covering the surface of this lung. Study the resultant phenomena of inflammation as exemplified by changes in the circulation, viz., the relaxation of the capillaries, the retardation of the flow, the greater vascularity of this part, the gradually increasing numbers of white blood-cells, the fixation of these cells to the walls of the vessels, and their final migration into the neighboring tissues.

4. Effect of Variations in Intrathoracic Pressure Upon the Bloodflow Through the Lungs. - With the help of the apparatus represented in Fig. 92 study the effect of the inspiratory increase and expiratory decrease in intrathoracic pressure upon the blood-flow. The large orifice of a bell-jar is closed with a rubber membranc. To its central 
area is attached a metal plate and ring, so that the membrane as a whole may be lowered and raised. The upper orifice of the bell-jar is closed by means of a rubber cork bearing a relatively narrow inlet tube. This chamber is traversed by a horizontal tube of very soft rubber, draining a receptacle filled with water.

Allow the water to flow steadily through this tube. Then lower the rubber membrane (diaphragm), thereby decreasing the pressure in the glass compartment (intrathoracic pressure). Note that the "pul-

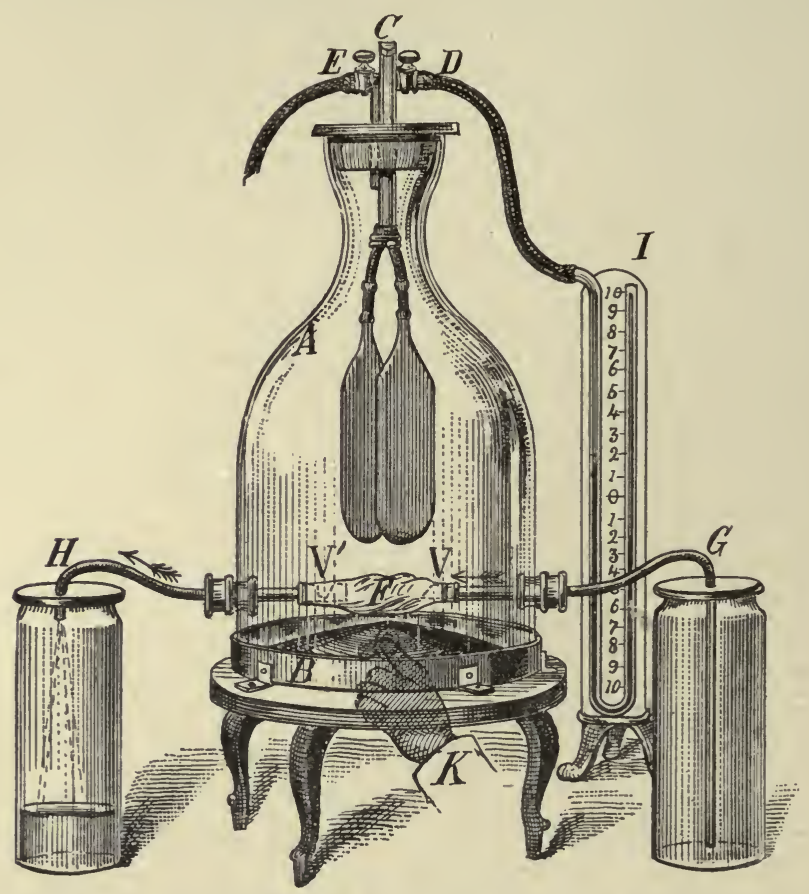

Fig. 92.-Device to Illustrate the Influence of the Respiratory Movements upon the Flow of the Blood through the Pulmonary Blood-vessels. (Hering.)

$A$, Bell jar; $B$, rubber membrane closing it; $V$, soft-rubber pouch to imitate the pulmonary blood-vessels; $G H$, arrangement for forcing water through $V$ under a constant pressure; $j$, manometer connected with "intrapleural space." On inspiration, produced by moving the rubber membrane downward, the intrapleural pressure is decreased. This gives rise to an aspiration which tends to pull the wall of $V$ outward, facilitating the flow from $G$ to $H$.

monary blood-bed," as represented by the thin rubber tube, is now large, allowing a free through flow. The opposite effect is produced by raising the diaphragm. The caliber of the pulmonary blood-vessels is then decreased and the pulmonary resistance increased.

5. Effect of Decreased Atmospheric Pressure.-Place a mouse under the bell-jar of an ordinary air-pump. Allow the pressure existing within this compartment to be recorded by means of a mercury manometer connected with the suction tube of the pump. Apply suction, lowering 
the pressure gradually to one-half and one-third of an atmosphere $(760 \mathrm{~mm} . \mathrm{Hg})$. Under normal conditions oxygen exerts a pressure of 20.94 per cent. of an atmosphere or about $153 \mathrm{~mm}$. $\mathrm{Hg}$. At 10 per cent. (one-half of an atmosphere) the animal will become restless and dyspneic, and at 7 per cent. (one-third of an atmosphere) lose consciousness and die. This pressure corresponds to the pressure prevailing at an altitude of 30,000 feet. 





\section{LESSON XXXI}

\section{RESPIRATION (Concluded)}

\section{ELIMINATION OF CARBON DIOXID AND CONSUMPTION OF OXYGEN}

1. The Elimination of Carbon Dioxid and Water.-Exhale repeatedly through a glass tube into a beaker filled with lime-water. Explain the resultant turbidity.

Procure a 4-ounce Woulffe bottle with three necks and the necessary delivery tubes and stoppers; three 5-inch calcium chlorid tubes with side tubes and perforated stoppers; a Geissler bulb with $\mathrm{KOH}$ and $\mathrm{CaCl}_{2}$ tubes, two small flasks with stoppers, and two glass tubes; a 2-liter bottle in which the animal is placed; and two 8-liter bottles.

The tubes containing the calcium chlorid should be put in the drying oven at a temperature of $100^{\circ}$ to $120^{\circ} \mathrm{C}$. for several hours. They are then cooled in a desiccator. Weigh two of them, marked $e$ and $f$. The Woulffe bottle and Geissler bulbs are filled with a 50 per cent. solution of $\mathrm{KOH}$. To the latter is attached the $\mathrm{CaCl}_{2}$ part and rubber connecting tubes, which are then clamped. The whole is weighed. The two flasks $b$ and $h$ are filled with a strong solution of $\mathrm{Ba}(\mathrm{OH})_{2}$. Weigh the bottle $d$ into which the animal is to be placed later on. Connect these parts and fill one of the siphon bottles (8 liters). Arrange the other $(k)$ at a distance of $1 \mathrm{~m}$. below the filled one $(i)$.

Place a white rat into the 2-liter bottle and weigh. Connect this bottle with the others. Start the siphon. Adjust the distance of the siphon bottles so as to give a sufficient ventilation to the animal (indicated by its rate of respiration). When the upper bottle has been nearly emptied, clamp the tube connecting it with the adjoining flask and quickly put the second (now filled) bottle in its place. Remove the clamp, and again siphon. Continue this procedure for about one hour.

At the end of this period clamp the siphon tube, turn the stoppers of the $\mathrm{CaCl}_{2}$ bottles, and disconnect the tubing. Weigh tubes $d, e$, $f$, and $g$. Obviously, parts $a, b$, and $c$ remove the $\mathrm{H}_{2} \mathrm{O}$ and $\mathrm{CO}_{2}$ from the air supplied to the animal, whereas parts $e, f$, and $g$ collect the $\mathrm{H}_{2} \mathrm{O}$ and $\mathrm{CO}_{2}$ given off by the animal in the course of this experiment. By weighing these parts before and after this test a means is provided for determining the amount of these excreta.

Determine the loss in weight suffered by the animal during this test. Ascertain how much $\mathrm{H}_{2} \mathrm{O}$ and $\mathrm{CO}_{2}$ left the animal during this period. Do these amounts correspond to the loss in weight of the animal? Explain.

2. The Consumption of Oxygen.-Fill the pressure tube $B$ with a solution of potassium pyrogallate, made by mixing 2 parts of a 25 per 
cent. aqueous solution of $\mathrm{KOH}$ and 1 part of a 5 per cent. aqueous solution of pyrogallic acid. The air must be analyzed as it passes into

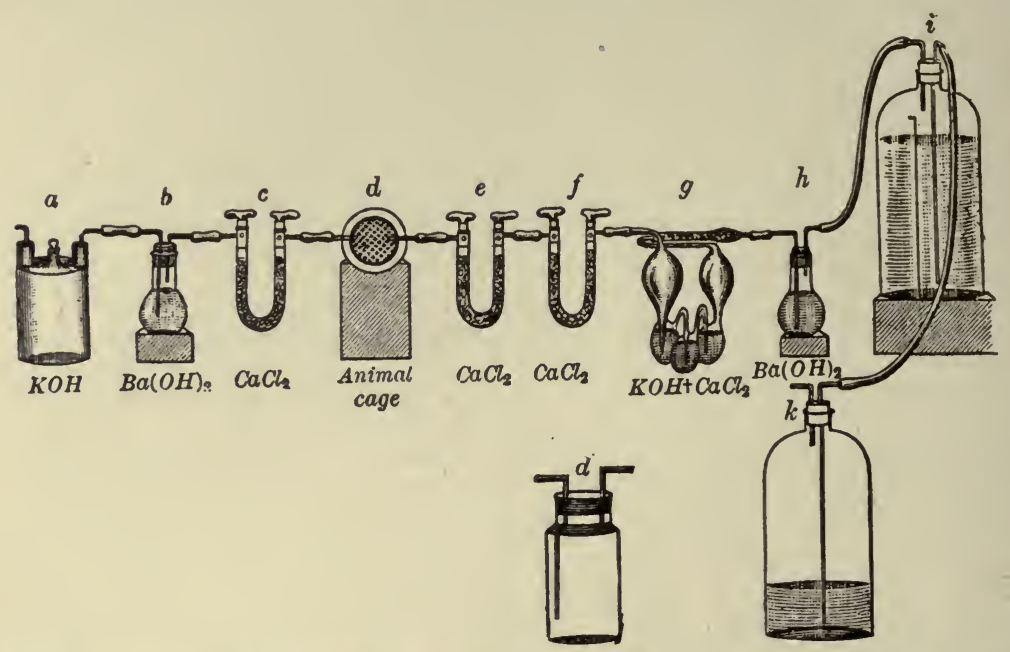

Fig. 93.-Apparatus for Estimating the $\mathrm{CO}_{2}$ and $\mathrm{H}_{2} \mathrm{O}$ of the Expired Air. (Hall.)

the apparatus represented in Fig. 93 and again as it leaves the siphon bottle. To accomplish this end fill the gas buret $A$ with water by

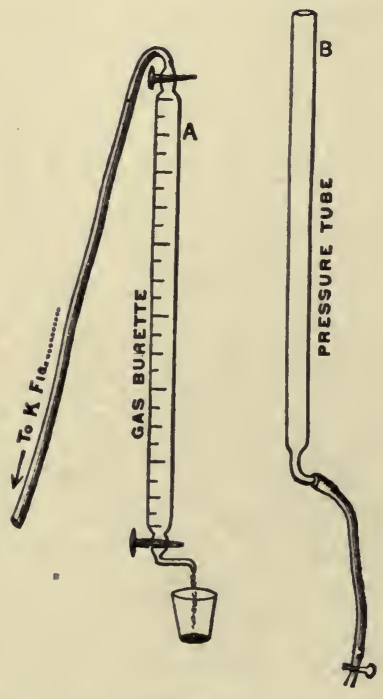

Position 1.

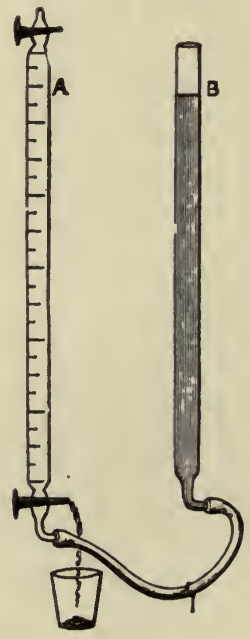

Position 2.

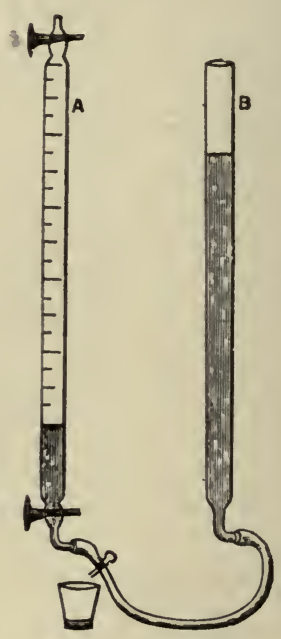

Position 3

Fig. 94.-Gas Burets for Determination of Oxygen. (Hall.)

suction. Connect its upper end with tube $K$ of the respiration apparatus (Fig. 93), and allow the siphoned air to displace the water in the 
buret. Turn the stop-cocks upon its two ends, and connect its lower end with the rubber tube of the pressure bottle $B$, containing the potassium pyrogallate. Expel the air from the connecting tube and turn the three-way stop-cock so that the pyrogallate may flow into the buret. Raise the pressure tube and finally clamp the connecting tube close to the buret. Turn the buret repeatedly (ten minutes) and open the clamp, allowing the potassium pyrogallate to take the place of the absorbed oxygen. Then remove the clamp, but allow the tubes to remain in connection for another ten minutes. At the end of this period adjust the level of the solution in the buret to the level of the solution in the pressure tube. Take the reading. Analyze the oxygen content of the normal air. Subtract the amount of oxygen of the respired air from that found in the normal air. The result corresponds to the amount of oxygen consumed by the animal. 




\section{LESSON XXXII}

\section{THE NERVOUS SYSTEM}

\section{REFLEX ACTION}

1. Histologic Study of Different Neurons.-Examine under the microscope motor neurons from the cerebral cortex, cells of Purkinje from the cerebellum, motor cells from the anterior horn of the spinal gray matter, and sensory cells from the spinal ganglion. Draw a diagram of each.

Orient yourself regarding the principal tracts of the spinal cord, the formation of the spinal roots and their function, and other data of general interest.

2. Dissection of the Nervous System of the Frog.-Kill a frog with ether. Make a median incision through the skin covering the skull cap. Hold the scalpel slantingly and perforate the bone about midway between the eyes, taking care not to penetrate too deeply. With a pair of small forceps cut away the bone around the perforation, enlarging the opening considerably. Having uncovered the white cerebral hemispheres and olfactory lobes, dissect backward until you have brought into view the rounded, grayish optic lobes or corpora bigemini. The cerebellum is rudimentary and occupies a position in front of these bodies. Identify the optic nerves.

Expose the entire spinal cord by breaking away the vertebræ along the dorsal aspect of the animal. Identify the spinal nerves and spinal roots.

3. Reflex Action.-Pith a frog and destroy its brain (not the spinal cord). Suspend the animal from a stand over a plate. Pinch the toes of one foot with the forceps. Observe that the foot is withdrawn from the seat of the stimulation by muscular activity.

Produce this reaction by immersing the foot in a weak solution of acetic acid. Repeat by applying the electrodes to the sole of the foot and stimulating with a brief tetanic current of moderate strength.

Destroy the spinal cord with a wire. Repeat the electric excitation and observe whether or no the different impulses so generated still induce motor responses. Draw conclusions regarding the part played by the spinal cord in this reaction.

Annotation.-The solution of acetic acid should be weak, possibly 3 drops of glacial acetic acid to about 20 c.c. of water. Strengthen it in case it should fail to stimulate. Immerse the foot in clean water after every stimulation. When the electric current is employed as a means of inducing reflex action, differentiate sharply between the local muscular contractions and those co-ordinated general contractions which eventually cause the removal of the foot from the seat of the stimulation.

In order to save material the student may omit destroying the spinal cord with the wire. This procedure, as may be surmised, destroys the spinal reflex ac- 
tions, because it produces a break between the afferent and efferent arcs of the reflex circuits.

4. Localization of the Reflex Center for the Hind Legs.-If the spinal cord has not been destroyed in the frog used for Experiment 3, this animal may now be employed for the following test: Suspend the brainless frog in the usual way. Open its abdominal and thoracic cavities and remove the viscera. Identify the vertebral column with its nine vertebræ. Produce a reflex by stimulating the sole of the foot electrically. Now make a transverse cut between the second and third vertebræ. Test the reflexes again. Cut between the third and fourth

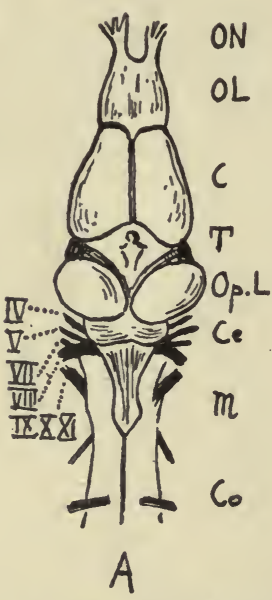

Fig. 95.-Diagrammatic Representation of the Brain of the Frog.

$O N$, Olfactory nerves; $O L$, olfactory lobe; $C$, cerebrum; $T$, tween brain; $O x L$, optic lobes; $C e$, cerebellum; $M$, medulla; Co, spinal cord. The cranial nerves are indicated by Roman numerals.

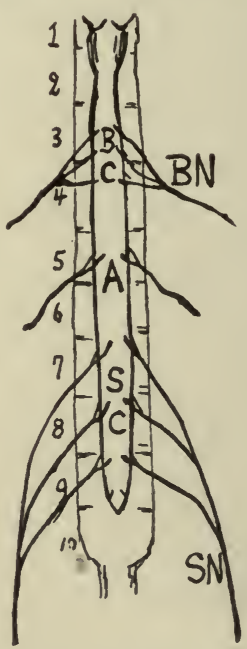

Fig. 96.-Diagram to Show the Position of the Reflex Centers in the Spinal Cord of the Frog.

$B C$ and $B N$, Brachial center and nerve; $A$, center for the parts of the trunk; $S C$ and $S N$, sciatic center and nerve. The numbers indicate the different vertebræ.

vertebræ and again test the reflexes. Continue this process until no longer able to elicit this reflex. At which level do you localize the sciatic center?

Annotation.-The frog possesses nine vertebræ. Its vertebral column ends at the dorsal prominence. From here the tenth vertebra or urostyle extends backward to the tip of the body. Notice that the three roots of the sciatic nerve enter the cord at different levels, the arrangement being such that a transverse cut made between the sixth and seventh vertebræ traverses the upper extent of the sciatic center, destroying the reflexes executed with the help of this nerve.

5. Localization of Function.-The same frog may be used for the following test: Identify the three roots of one sciatic nerve and place them in loose ligatures. Place the frog upon a moistened plate and stimulate each root separately with single induction shocks of very 
moderate intensity. Divide them and stimulate the distal end of each. Observe that they innervate different groups of muscles.

A similar localization of function may be detected in the sciatic center itself. Very fine needle electrodes should be used in mapping out this area.

6. Summation of Afferent Impulses.-Destroy the brain of a frog (not the cord). Adjust two thin copper wires to one foot about $1 \mathrm{~cm}$. apart and connect them with the secondary coil of an inductorium. Stimulate with a subminimal-reflex induction shock, $i . e$., with one which does not evoke a reflex, but may cause a local muscular reaction. Stimulate with two or three of these shocks in quick succession, and observe the reflex action ultimately resulting in consequence of this summation.

7. Effect of Thermal Stimuli.-Remove the wires from the frog used in Experiment 6. Dip the foot at intervals into water of $10^{\circ}, 20^{\circ}$, and $30^{\circ} \mathrm{C}$. Which is the most efficient stimulus? Finally, immerse the foot in cold water, which is then heated gradually until an intense reflex is evoked.

8. Spreading of Reflexes.-Apply the electrodes to the foot of this frog and stimulate first with a weak current and then with a strong current. Note that the weak stimulation gives rise to a perfectly localized reflex, whereas the strong stimulation evokes, in addition, movements of the other leg; trunk, and forelimbs. In other words, the strong stimulation causes the primary impulses to spread to other reflex circuits.

Hold the foot of one leg between your fingers. Place a small piece of filter-paper moistened with moderately dilute acetic acid, upon the skin of the ventral aspect of the thigh of the same leg. Observe that the impulses so elicited eventually involve other reflex circuits, causing the opposite leg to be moved. Naturally, if the filter-paper is brushed away by these movements, this result has a purely mechanical cause. Immerse the frog in fresh water.

9. Reflex Time.- Suspend the frog in the usual way and stimulate the sole of one foot several times with weak and strong electric currents. Count in each case the number of seconds elapsing between the moment of stimulation and the onset of the reflex action. This interval is the so-called reflex time. What is the relationship between this period and the intensity of the stimulus.

10. Inhibition of Reflexes Upon Central Paths.-Expose and ligate the sciatic nerve of one side. Divide the nerve distally to the ligature. Apply the electrodes to the central end of this nerve. Immerse the foot of the opposite leg in a weak solution of acetic acid and simultaneously stimulate the central end of the sciatic nerye with a weak tetanizing current. Note the resultant inhibition of the reflex. At what point of this reflex system do the impulses from the central end of the opposite sciatic nerve interfere with the impulses from the foot immersed in the acid? 





\section{LESSON XXXIII}

\section{THE NERVOUS SYSTEM (Continued)}

\section{REFLEX ACTION. REMOVAL OF CEREBRUM}

1. Inhibition of Reflexes by Higher Centers.-Etherize a frog. Make a median incision through the skin covering the skull-cap. Perforate with the point of a knife and enlarge the opening by means of a pair of forceps. Identify the cerebral hemispheres and remove them. After an interval suspend the frog and determine the reflex time in the usual way.

Sprinkle a few crystals of sodium chlorid upon the upper surface of the optic lobes, and again ascertain the reflex time. Since these bodies possess an inhibitor action upon spinal reflex action, this means of stimulation will tend to lengthen this period.

Remove both optic lobes, and again determine the reflex time. Obviously, their removal must destroy this inhibitor influence and intensify spinal reflex action.

2. Exaggeration of Reflexes by Means of Strychnin.-Inject a drop or two of a 0.5 per cent. solution of sulphate of strychnin into the dorsal lymph-sac of a frog. After a few minutes stimulate the foot of this frog mechanically. Repeat at brief intervals, noting the progressive character of the muscular seizures. Blow your breath at the frog or tap upon the table upon which it is resting. Upon which elements of the reflex circuit does the strychnin exert its action?

3. Reflexes in Man,- Let the subject open his mouth. Touch the uvula with the end of an aseptic glass rod. It will rise. Touch the fauces. The response may be either a movement concerned with the act of swallowing or the gagging reflex, an act tending to protect the digestive tract.

Make a sudden movement in front of the eyes of the subject as if you were going to strike him in the face. The eyelids are closed. Touch the outer surface of the cornea of the subject with a cotton fiber. An immediate closure of the eyelids is the result. In all these instances, however, the character of the reflex may be modified volitionally (inhibition by the cerebrum).

Shield the eye of the subject for a few seconds with your hand. Suddenly withdraw the latter, allowing light to enter the pupil. Observe the decrease in the size of this orifice, brought about by the contraction of the circular muscle cells of the iris (light reflex). Request the subject to accommodate alternately for far and near objects. The pupil is enlarged during far vision and constricted during near vision (accommodation reflex). Pinch the skin of the neck. The pupil will dilate (ciliospinal reflex). 
A simple secretory reflex may be produced by moistening the mucous lining of the mouth with a few drops of very dilute acetic acid or by chewing a piece of rubber or paraffin.

The sneezing reflex may be elicited by touching the mucous lining of the nasal cavity with a few cotton fibers. Inhibit this reflex by pressing upon the upper lip with the index-finger.

4. Tendon Reflexes in Man.-Cross your legs, allowing one leg to hang perfectly free. Let the assistant strike the patellar ligament of the free leg with the outer margin of his hand. Note the contraction of the quadriceps muscle and the upward kick of the leg and foot. During this test the subject should be perfectly inattentive, otherwise cerebral inhibition will result.

-Interlock the index-fingers of your hands. While you make a forced effort to separate these fingers let the assistant elicit the patellar reflex. Note that the kick is now much stronger than before (reinforcement of reflexes). Explain.

Stand beside a low chair with your leg resting upon it. Let the assistant strike the tendo achillis. The foot will be extended, owing to the contraction of the gastrocnemius muscle.

While the ankle-clonus is not observed in bealthy persons, the student should familiarize himself with the method employed in eliciting it. The patient is seated and rests his leg upon a chair of equal height, allowing the foot to project beyond its edge. Steady the leg with your left hand and with your right hand suddenly flex the foot upon the leg, so as to put the tendo achillis on the stretch. A series of clonic contractions of the corresponding muscles will result, in persons afflicted with certain spinal diseases.

Another peculiar phenomenon noted in certain spinal diseases is the so-called Babinski phenomenon. Under normal conditions the tickling of the sole of the foot results in a flexion of the toes upon the foot (plantar reflex), whereas under certain abnormal circumstances the first toe may be extended and the others flexed.

5. Muscle Tonus.-Etherize a frog under a bell-jar. When all sensibility has been lost, open the abdomen. Place the sciatic nerve of one side in a loose ligature. Suspend the frog in the usual way. Note that the legs are held in a position intermediate between complete relaxation and contraction (tonus). Divide the sciatic nerve previously placed in the ligature. Observe that the corresponding leg now assumes a more dependent position, $i$. e., relaxes more completely.

Remove the skin from the normal leg, and again note the position of this leg. It will now assume the level of the opposite leg, the sciatic nerve of which has been divided. Explain this result, making use of the contention that the tonus of skeletal muscles is dependent upon afferent stimuli derived from the integument.

6. Threshold of Stimulation.-Pith a frog. Carefully expose one sciatic nerve in the thigh. Determine the least strength of tetanizing current which will cause a spreading of reflexes when applied to the skin 
of the foot. Apply the same stimulus to the trunk of the sciatic nerve. The intensity of the stimulus required to evoke reflex action is usually less when applied to the sense organs.

Make a median incision through the skin covering the dorsal aspect of this frog. Raise the skin and identify one of the many nerve-fibers crossing the dorsal lymph-space to innervate the skin overlying. Cut out a piece of skin about $1 \mathrm{~cm}$. square, containing the terminals of this nerve. Raise this skin-flap, but allow it to remain in connection with the body by means of the nerve. Determine the least strength of stimulus required to cause a reflex movement when applied to the surface of this flap of skin Repeat, applying the electrodes to the aforesaid nerve. As a rule, the threshold value of the current will be found to be lower in the former instance.

7. Effect of Removal of the Cerebrum.-Etherize a male frog under a bell-jar. When completely insensitive make a median incision through the skin covering the skull-cap and perforate the skull with the point of a scalpel. Enlarge the opening and remove the cerebral hemispheres and olfactory lobes. Note that the junction between the cerebrum and the optic lobes is indicated externally by an imaginary line drawn through the anterior margins of the ear drums. Work rapidly but carefully. Bring the edges of the wound together by means of two or three sutures, and moisten the skin repeatedly with fresh water. Allow the animal to recover fully. Carefully study its behavior:

(a) What is its posture? Pass your hand in front of its eyes. Is it, made to move thereby? Repeat this test upon a normal frog. Compare.

(b) Gently pinch the toe of the decerebrated frog. Do you note any abnormality in its manner of jumping? Place the animal in water. Is its power of swimming affected in any way? Does it retain its upright position?

(c) Place the frog upon its back. Does it right itself ?

(d) Place the frog upon a somewhat roughened flat surface. Tilt the board gradually and note how well it adapts the axis of its body to the surface. Repeat this test by placing the frog upon a small Ferris wheel. Gradually turn the wheel, noting that the frog attempts to reach the top by moving against the direction of the rotation. Repeat this test by placing the frog upon a rotating surface arranged horizontally. The long axis of its body will then be bent against the direction of the rotation (compensatory movements of equilibration).

(e) Hold a small tuft of cotton moistened with a few drops of acetic acid in front of its nostrils. The frog will make protective movements with its forelimbs and move away from the seat of the stimulation (trigeminal reflex).

(f) Place a narrow board between the frog and an incandescent light. Force the frog to jump toward the light. It will avoid the object casting the shadow (retinal reflex).

(g) Pass your index-finger over the skin covering the dorsal surface 
of this frog. Observe that the decerebrated animal produces a peculiar sound whenever touched. The same result may be obtained by grasping the frog in such a way that your thumb and index-finger come to lie laterally upon the abdomen. Stimulate a normal frog in the same manner. Compare.

Annotation.-The test described last is one of the best means of ascertaining the function of the cerebrum. In the normal animal the act of croaking is under the control of the cerebral hemispheres, $i . e$., it is an associated act. Consequently, it will be almost impossible to evoke it by inadequate stimuli. The removal of the cerebrum, on the other hand, changes this complex act into a simple reflex. The influence of the higher centers having been destroyed, it may then be evoked by ordinary stimuli in a reflex way.

A male frog may be recognized by the cushion-like thickening at the base of the innermost digit of the hand. Besides, the male rana esculenta possesses a bladder-like resonating pouch on each side of the mouth.

Attention should also be called to the fact that an animal without cerebral hemispheres is incapable of "feeling." The sensorium ceases to exist after the removal of these structures, and only the ordinary reflex sensory mechanism remains behind.

8. Influence of the Cerebrum.-Place a normal frog in a basin filled with water. Warm the water slowly, and note that the frog will make complex efforts to escape as soon as the temperature of this medium has risen to about $25^{\circ} \mathrm{C}$.

Place a frog, the cerebrum of which has been destroyed, in cold water. Warm the water to $40^{\circ} \mathrm{C}$., and observe the reflex movements resulting in consequence of the thermal stimulation. While this animal may escape from the basin, this result is acsidental, and is due solely to the reflex contractions of the muscles. 




\section{LESSON XXXIV}

\section{THE NERVOUS SYSTEM (Continued)}

\section{STIMULATION OF THE CEREBRUM. THE FUNCTION OF THE ROOTS OF THE SPINAL CORD}

1. Cerebral Localization.-Procure a pair of adjustable electrodes and connect them with the secondary coil of an inductorium. Arrange the electric apparatus for stimulation with a quickly interrupted current. Anesthetize a cat and maintain the anesthesia throughout the following experiments: Perform tracheotomy. Place the animal on its side. Make a median incision through the skin covering the skullcap, and separate the edges of the temporal muscles from the bone underneath. Adjust a trephine, about $1.5 \mathrm{~cm}$. in diameter, to the anterior area of the right parietal bone at a distance of $0.5 \mathrm{~cm}$. from the median line. Carefully work the trephine until it has penetrated the skull. Do not press upon it heavily so as not to break through suddenly, piercing the substance of the cerebrum. Remove the round plate of bone with a pair of forceps. Apply dry cotton, and stop the bleeding by pressing soft wax against the edge of the cut bone. Identify the dura mater and its blood-vessels.

Observe that the surface of the dura rises with every systole of the heart and also during inspiration. Insert a thistle tube in the trephine opening. Fill it partly with warm saline solution and connect it with a recording tambour. Register these pulsations upon the smoked paper of a kymograph. The cranial cavity is in this way converted into a plethysmograph, registering the cardiac and respiratory changes in the volume of the brain.

Remove the thistle tube and enlarge the trephine opening by means of a pair of bone forceps until the crucial area of the cerebrum has been completely uncovered. Stop the bleeding by means of wax and cotton tampons. Incise the dura mater, noting the escape of liquor cerebrospinalis. ' Reflect the dura and expose the surface of the cerebrum along the crucial sulcus (fissure of Rolando). Place the animal on its right side and unfasten the left fore- and hind limbs.

Using Fig. 97 as a guide, stimulate the surface of the cerebrum in the vicinity of the crucial sulcus with a weak tetanizing current. If no results are obtained, increase the strength of the current gradually and lessen the depth of the narcosis. Analyze the movements resulting in consequence of the stimulation of these different areas.

2. The Function of the Roots of the Spinal Cord.-Close the wound by means of a continuous suture. Make a median incision through the skin covering the spinous processes of the lumbar vertebræ. By means of forceps separate the fascia and muscle tissue from these processes. 
Stop bleeding by tampons, torsion, and ligation of the blood-vessels. Expose the laminæ of several adjoining vertebræ. Cut through them and remove the dorsal wall of the spinal canal of this region. Take care not to injure the spinal nerves.

Incise the dura mater. Expose the spinal cord and identify the anterior and posterior roots of one of the spinal nerves. Isolate these roots and place each in a loose ligature. Stimulate each with a weak tetanizing current. Eventually tie these ligatures, the one upon the anterior root close to the cord, and the one upon the posterior root far

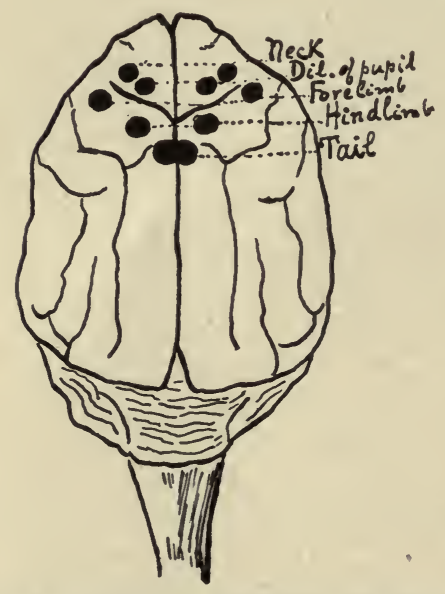

Fig. 97.-Diagram Showing the Motor Points in the Cerebrum of the Dog.

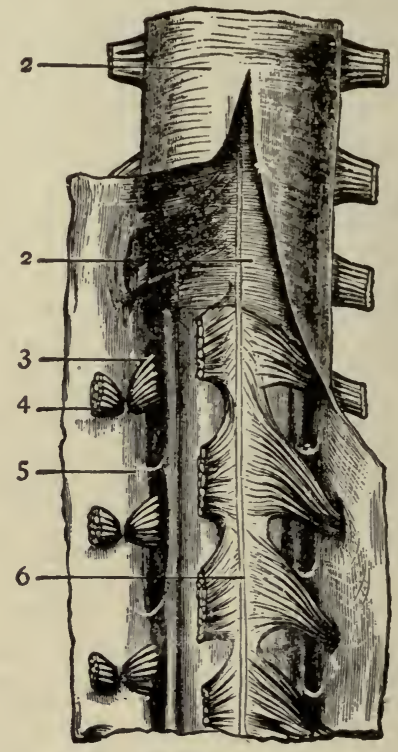

Fig. 98.-The Membranes of the Spinal CORD.

1, Dura mater; 2, arachnoid; 3 , posterior root of spinal nerve; 4 , anterior root of spinal nerve; 5 , ligamentum dentatum; 6, linea splendens. (After Ellis.)

away from the cord. Stimulate the distal end of the former with a weak tetanizing current. Repeat the stimulation upon the central end of the latter. Tabulate the results, and determine the direction of conduction in each root.

Kill the animal by an overdose of ether. Remove a segment of the spinal cord, noting the size and shape of the subdural space, and the manner in which the spinal nerves are enveloped by dura.

Remove the brain. Identify its different parts, and especially those to which attention has been called in the lectures. 




\section{LESSON XXXV \\ THE NERVOUS SYSTEM (Concluded)}

REACTION TIME

1. Reaction to Touch.-Arrange the electric apparatus for stimulation with single induction shocks. Insert a signal and two simple keys in the primary circuit of an induction apparatus, and connect a pair of platinum electrodes with the secondary coil. Arrange to record the movements of the signal. Add a tuning-fork. The latter should be fastened to a separate stand and be allowed to record from left to right, $i$. e., against the direction of rotation of the drum of the kymograph. Let the subject hold the electrodes against his tongue with his left hand, while his right hand grasps the handle of one of the two keys. Let the observer then spin the drum and close the other key. As soon as the subject feels the make shock, let him break the current by opening his key. Draw ordinates to the curves and determine the time which has elapsed between the make and the break of the current. Repeat this experiment several times and ascertain the average reaction time.

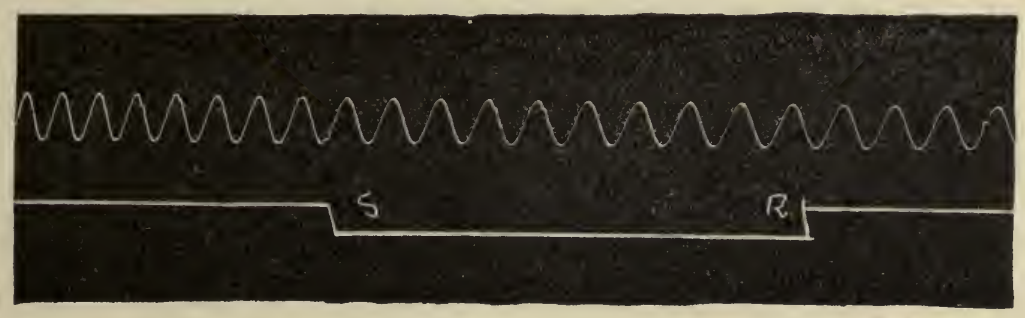

Fig. 99.-Reaction to Touch.

Record of signal below that of tuning-fork (1 $1 \frac{1}{20}$ sec.). $S$, Moment of stimulation; $R$, moment of reaction.

Repeat this experiment with a stronger stimulus. What relationship exists between the strength of the stimulus and the length of the reaction time?

2. Reaction to Light.-Arrange 5 dry cells in series, their total strength amounting to about 7 volts. Connect these cells with a small incandescent lamp (5 or 6 volts), a signal, and two simple keys. Arrange to register the movements of the signal above the record of a tuning-fork vibrating in hundredths of seconds. Let the subject hold the bridge of one key in the position of closure. Shield the other key and apparatus from the subject by a large cardboard. Let the observer spin the drum and suddenly light the lamp by closing the bridge of the second key. The subject should open his key as soon as he perceives the light. Determine the average reaction time of several tests of this kind. 
3. Reaction to Sound.-Arrange in series 2 or 3 dry cells, a simple key, a signal, a hammer, and a metal plate. Arrange to record the movements of the signal and of a tuning-fork. Let the subject hold the key closed. Let the observer spin the drum and sharply tap with the hammer upon the plate, thereby making the circuit. Let the subject open the simple key as soon as he hears the sound. Determine the average reaction time of several tests of this kind.

4. Reaction Time with Choice.-Arrange the electric apparatus for stimulation with single induction shocks and insert a signal and two simple keys in the primary circuit. Connect the secondary coil with a rocking bridge or mercury pole changer, and, in turn, each pair of connectors with electrodes. Let the subject hold the electrodes to his tongue, one on each side of it, and instruct him to open his key only when the right side of his tongue has been stimulated. Shield the apparatus by a large cardboard. Set the rocking key for stimulation of either side of the tongue. Spin the drum and close your key. Repeat several times, changing the point of stimulation, so that the subject is forced to judge which side has been stimulated. Does choice prolong the reaction time?

5. Patellar Reflex Time.-Let the subject be seated and cross his legs. Adjust to the thigh of the crossed leg a rubber cuff, and connect the latter by means of rubber tubing with a recording tambour. Adjust a tuning-fork upon a separate stand and arrange it to register its vibrations from left to right, $i . e$, against the direction of rotation of the drum of the kymograph. Spin the drum and tap the patellar ligament of the subject, while he endeavors to reinforce the patellar reflex by simultaneous efforts. The record of the tambour will show two oscillations, namely, a wave due to the blow upon the ligament and one caused by the contraction of the muscles of the thigh. Draw an ordinate at the beginning of each wave, and determine the time which has elapsed between the moment of stimulation and the reaction.

6. Reflex Winking Time.-Connect the upper eyelid of the subject by means of a fine thread and soft wax with a recording lever. Insert. a signal in the primary circuit of an induction apparatus, and place the writing point of the signal vertically below that of the recording lever. Allow a tuning-fork to record below the signal. Place upon the lower eyelid a pair of stimulating electrodes. While the drum is revolving at a rapid rate stimulate with a single make or break shock of suitable strength. Draw ordinates and measure the interval between the moment of stimulation and the moment when the upper eyelid began to react. 




\section{LESSON XXXVI}

\section{THE SENSE ORGANS}

\section{CUTANEOUS AND MUSCULAR SENSATIONS}

1. Histologic Examination of Tactile Corpuscles.-Place different tactile corpuscles under the low and high powers of a microscope and study their structure.

2. Touch Localization.-Touch the skin of the hand of the subject with the pointed end of a pencil, his eyes being kept closed throughout this experiment. Let him then place the blunt end of a pencil upon the area stimulated. Measure with a millimeter scale the error made by him. Repeat the foregoing experiment upon the forearm and cheek of the same subject.

Touch the skin of the subject twice in quick succession, selecting for the two stimulations either precisely the same point, or two points lying close to one another. Let the subject state whether two areas have been stimulated or only one.

3. Touch Discrimination.-By touching different areas of the surface of the hand, arm, and face with the points of a caliper ascertain how

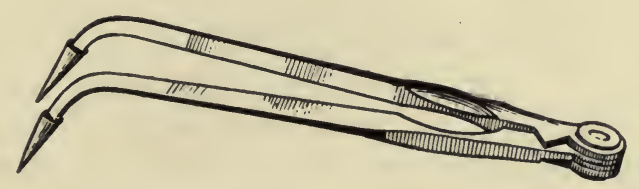

Fig. 100.-Esthesiometer with Guarded Points. (Stirling.)

widely this instrument must be opened in order that its points may be felt as two. Record in millimeters the results for each part stimulated, and compare them, paying special attention to the relative sensitiveness of the lips, cheeks, tongue, and the flexor and extensor surfaces of the arm. In the latter case observe that the sensibility increases in the direction of the fingers and also in the transverse direction rather than along the longitudinal axis of the limb.

4. Action of Cocain.-Press the point of a needle upon the tongue and note the degree of pressure necessary to produce a distinct sensation of pain. Touch this area with the end of a camel's-hair brush, moistened with a 4 per cent. solution of cocain. What changes do you note?

5. Aristotle's Experiment.-Cross the right middle and index-fingers and place them upon the palmar surface of the left hand. Place a small shot between them and roll it about in the palm of the hand. Describe the sensation. 
Cross the fingers in the same way and rub them against the tip of the nose. Describe the sensation.

Annotation.-Ordinarily the tactile corpuscles upon the outer surface of the index-finger and inner surface of the middle finger act in unison, producing harmonious impressions. If the corpuscles upon the inner aspect of the index-finger are now brought in relation with those upon the outer aspect of the middle finger, two impulses must result in consequence of the stimulation of dissimilar tactile points.

\section{Peculiar Phenomena and Illusions of Touch and Pressure.-} Place the cross-section of a tube against the skin of the forearm. An impression of a transversely oval object is produced thereby.

Separate the points of a compass about $2 \mathrm{~cm}$. and draw them in a straight line downward across the skin of the forearm, wrist, palm of hand, and fingers. A sensation of a single line opening up into two is produced; moreover, these lines appear to be more widely apart at the wrist, to converge in the palm, and to diverge toward the tips of the fingers.

Close your eyes, and follow the circumference of a round object first with a short rod and then with a long rod, held between your fingers in the usual way. The object will appear to be much larger when outlined with the short rod.

Apply the points of a compass simultaneously to the skin of the subject, his eyes being closed. Allow the subject to obtain a clear impression of the distance between the points. Apply only one of the points, and move this point rapidly an equal distance across the skin. In the former instance the distance will seem grester.

Make a knot in a coarse thread about $30 \mathrm{~cm}$. in length. Allow the subject to hold the knot between the thumb and index-finger of his right hand, his eyes being closed. Pull the thread through his fingers first slowly and then more quickly. Is the subject capable of estimating the length of the thread?

Apply a comb to the dorsal surface of the subject's hand, his eyes being closed. Ask him to indicate the length of the comb actually applied. It will appear shorter to him than it really is. Draw the same distance of comb slowly across the surface. It will now seem longer than it actually is, because movement leads to an exaggerated sensation of length.

Draw the head of a pin rapidly to and fro across the skin of the forearm of the subject, his eyes being closed. The subject will perceive the motion some time before he is able to determine its direction.

Touch your forehead with your finger. The finger "feels" the forehead. Rapidly draw the finger across the skin of the forehead. The sensation will now be referred to the forehead.

7. Adaptation to Touch Sensations.-Place an object, such as a cork, upon the skin of the forearm. The initial sensation of pressure will gradually give way to an indifferent sensation.

Dip your index-finger into a tube filled with mercury. Presently 
only the sensation of pressure at the surface of the mercury will remain behind; in fact, eventually even the latter will appear only when the finger is moved.

8. Touch Sensations Modified by Movement.-Touch the skin of the dorsal surface of the arm with the tip of your index-finger. Note the quality of the sensation. Draw the finger slowly across this surface. The sensation of touch is changed into one of stroking, due in all probability to the activation of the nerve plexuses investing the roots of the hairs.

Feel any surface with the tip of your index-finger. Draw the finger across the surface. Note that the sensation of simple contact is now amplified by sensations of motion and space.

9. Projection of the Sensations of Touch.-Hold a metal rod between your fingers and draw its end across a roughened surface. The sensation will be referred to the end of the rod, $i$. e., be projected beyond the skin.

Dip the elbow in cold water. The initial sensation of cold at the point of eontact with the water will soon give way to a similar sensation in the region innervated by the ulnar nerve.

10. Mechanical Stimulation of the Hot and Cold Spots.-Close your eyes and instruct the assistant to touch the dorsal aspect of your hand with the blunt point of a pencil. In certain areas you will obtain a sensation of cold, and in others, of heat. These spots are separated by areas in which no sensations of temperature are evoked.

Allow the assistant to draw the point of a pencil slowly across the surface, noting that distinct points of cold flash out. Sensations of heat are not so easily elicited.

11. Thermal Stimulation of the Hot and Cold Spots.-Employ a metal rod, about $10 \mathrm{~cm}$. in length and $1 \mathrm{~cm}$. in diameter. Its pointed end projects from a covering made of rubber tubing. Cool the rod in ice-water and map out a circumscribed area upon the dorsal aspect of the subject's hand. Mark the cold spots with black ink. Warm the rod to $70^{\circ} \mathrm{C}$. and proceed as before, marking the warm spots in red ink. Observe that the latter are less easily found and that their stimulation is followed by a longer latent period.

12. Chemical and Electric Stimulation of the Temperature Spots.Apply objects of different heat-absorbing power to the skin, such as wool and a piece of metal. The latter feels colder because it gives rise to a greater loss of heat, thereby stimulating the cold spots.

Rub menthol upon the skin of the hand or forehead. A sensation of cold is obtained because this agent renders the skin hyperesthetic.

Identify a cold spot upon the dorsal surface of the hand. Employ two electrodes, one pointed and the other flat. The former is applied to the cold spot and the latter elsewhere upon the hand. Stimulate with a weak induction current until a distinct sensation of cold is obtained.

13. "After-images" of Temperature.-Place a cold coin on the 
forehead or on the palm of the hand for about half a minute. Does the sensation of cold continue even after the coin has been removed, allowing the temperature of the skin to rise? Repeat this test with a slightly heated coin.

14. Acuity of the Temperature Sense.-Insert the index-finger in a beaker filled with water of $30^{\circ} \mathrm{C}$. Raise the temperature of the water quickly a few tenths of a degree. How small a difference are you able to perceive?

15. Temperature Contrast.-Place the index-finger of your right hand in water of $40^{\circ} \mathrm{C}$. and the index-finger of your left hand in water of $20^{\circ} \mathrm{C}$. Wait a short time until the initial sensations of warmth and cold have become less intense. Transfer both fingers into water of $30^{\circ} \mathrm{C}$. Note that the right finger feels cold because heat is lost by it, whereas the left finger feels warm owing to a certain stagnation of its heat.

Place one index-finger in water of $32^{\circ} \mathrm{C}$. and the other in water of $45^{\circ} \mathrm{C}$. Wait half a minute and transfer both in water of $10^{\circ} \mathrm{C}$. Analyze the sensation.

16. Thermal Illusions.-Employ two disks of metal of equal size, one warm and the other cold. Place them successively upon the skin of the subject, whose eyes are closed. The cold disk will feel heavier than the warm one.

17. Pain Spots.-Map out the dorsal surface of the hand of the subject with the point of a sewing needle, noting the points where a distinct sensation of pain is perceived.

18. Discrimination of Weight: Weber's Law.-Place a small box containing 10 shot upon the palmar surfaces of the tips of the middle and index-fingers of the subject. Support his hand at the wrist, and ask him to close his eyes. When he has obtained a clear impression of the weight, add or subtract shot until he notices a distinct difference. Repeat this experiment several times and obtain a mean value for the number of shot added or removed. Repeat this test with 30 and 50 shot respectively in the box. Tabulate the results.

19. Relation of Weight to Area Stimulated.-Place two objects of equal weight but unequal size upon the dorsal aspect of the subject's hand, his eyes being closed. Most generally, that weight will be thought to be heavier which presents a greater surface to the skin, $i$. e., stimulates the largest number of tactile corpuscles.

Request the subject to lift three cylinders of equal weight, but unequal size, and determine which is the heaviest. The largest cylinder is usually thought to be the heaviest.

20. Illusions Relating to Weight.-Lift an object first rapidly and then slowly, and note that its weight seems less in the former instance. Lift a certain weight with one hand while clenching the other. The weight seems lighter in consequence of the simultaneous effort.

21. Simultaneous Movements.-Stand erect before a blackboard. Close your eyes and draw with both hands placed at the same height two 
leaf patterns of equal size. Draw from left to right with a "free hand" motion of the arms, produced by simultaneous impulses directed equally to the two sides. Note the relative size and position of the figures.

Repeat this experiment, but place one hand about $12 \mathrm{~cm}$. above the other. Obviously, the muscle sense is not well trained in the average person.

22. Sensation of Motion at the Elbow.-Place the forearm upon a board which it is possible to move, thereby imitating the flexion of the forearm upon the arm. Close the eyes. While an assistant raises or lowers the free end of the arm-board, state when you perceive a distinct motion of your forearm. How great an angular movement is necessary in order to produce a sensation of motion?

23. Paradoxic Resistance.-Fasten a weight to a string about $2 \mathrm{~m}$. in length. Close your eyes and grasp the string anywhere, suspending the weight in space. Quickly lower the weight upon a felt cushion. When contact is made a definite sensation of resistance arises, as if the hand were supported by a rod. 





\section{LESSON XXXVII \\ THE SENSE ORGANS (Continued)}

TASTE, SMELL, HEARING

1. Structure of the Taste-buds.-Study histologic preparations of the taste-buds.

2. Distribution of Taste.-Place a crystal of cane-sugar upon the tip of the tongue. Note that there is a definite latency caused by the fact that solid substances cannot be tasted. They must first go into solution.

Place a crystal of cane-sugar upon the tip of the tongue and another upon its posterior area. Where is the sweet taste most pronounced?

Employ a solution of sulphate of quinin (bitter), a 5 per cent. solution of cane-sugar (sweet), a 10 per cent. solution of $\mathrm{NaCl}$ (saline), and a 1 per cent. solution of acetic acid (sour). Apply these solutions to different parts of the tongue by means of a camel's-hair brush, and observe where each is tasted most acutely.

3. "Threshold Value" of Taste.-Moisten the tongue with $\frac{1}{2}$ teaspoonful of a $1: 1000$ solution of cane-sugar. Do you perceive a sweet taste? Rinse the mouth and repeat the experiment with solutions of the following strengths: $1: 800,1: 600,1: 400$, and $1: 200$. Which solution produces the least perceptible sweet taste? How does the acuity of taste in smokers compare with that in non-smokers?

4. Taste Reaction of Single Papilla.-By using a lens select a fungiform papilla near the tip or side of the tongue. Apply to it the tip of a camel's-hair brush moistened with one of the fluids provided for this purpose, viz., weak and strong solutions of cane-sugar, sodium chlorid, tartaric acid, and quinin. The subject should indicate the taste which he perceives. Does the papilla experimented with respond to more than one of the agents used? Test other papillæ in the same manner. Explain.

If a papilla be found which reacts to bitter, paint it with a solution of cocain. To another, which is particularly responsive to sweet, apply a saturated alcoholic solution of gymnemic acid. . Note the result in each case.

5. Electric Stimulation. Inadequate Stimuli.-Connect two small zinc electrodes with a series of 4 dry cells. Apply one of the electrodes to the upper and the other to the lower surface of the tongue. An acid taste will be obtained at the positive and an alkaline one at the negative pole. The objection that electrolysis is the cause of these sensations may be met by employing non-polarizable electrodes. Moreover, even single shocks which cause practically no electrolysis, give rise to taste sensations. 
6. Elimination of Sweet and Bitter.-Apply a 5 per cent. decoction of gymnema sylvestris to a limited area of the tongue. Twenty to thirty seconds later rinse the mouth thoroughly and test the taste with the solutions used for Experiment 2. What solutions remain effective?

7. Structure of Olfactory Cells.-Study hitologic preparations of the olfactory area.

8. Distribution of Olfactory Cells.-Place a glass funnel over some odoriferous substance. Insert the tip of the funnel first into the lower posterior and then into the upper anterior region of the nasal cavity. Which region is the more sensitive to the odor? Where is the olfactory area located?

9. Olfactory Latency. - Smell oil of cloves held close to the nose, and determine the time intervening between this act and the perception of the sensation. Give a reason for this latency.

10. Olfactory Fatigue.-Smell tincture of camphor, tincture of iodin, or oil of cloves with one nostril until the olfactory cells have been fatigued. Note the time it takes for the fatigue to set in and for the acuteness of smell to be re-established.

11. Qualitative Changes Before Exhaustion.-Inhale oil of cloves through one nostril and observe the changes which occur in the quality of the sensation before it ceases.

12. "Threshold Value" of Sounds.-Determine the greatest distance at which the subject can still hear the tick of a watch placed on the level of his right or left ear, the other ear having been closed with cotton. To avoid inattention the subject should shut his eyes. When just at the threshold of audibility the sound varies greatly in its intensity.

Place the handle of a vibrating tuning-fork upon the head. Note the intensity of the sound, and then remove the fork quickly before the sound ceases completely. Observe that the change to complete silence seems much greater than the apparent low intensity of the sound would justify.

13. Auditory Fatigue.-Insert the ends of a Y-shaped rubber tube into the openings of the ears. Place a vibrating tuning-fork upon the tube in such a way that the sounds seem equally intense to both ears. Remove the tuning-fork. After a brief interval occlude the tube on one side by pinching it, and place the vibrating tuning-fork in its former position. When the sound has nearly ceased to be audible, open the pinched tube. The sound now appears to be much stronger in the rested ear than in the other.

14. Location of Tones.-Place the handle of a vibrating tuning-fork upon the top of the head. In what part of the head does the sound seem to be localized? Close one ear and observe the change in the apparent localization of the sound. Explain this phenomenon.

Note the effects produced by placing the tuning-fork upon different parts of the head.

Place the tuning-fork upon the teeth. Close one ear and note the apparent change in the location of the sound. 
Place first the right side and then the left side of your head flat against a pillow. Do you hear the sounds of your heart? In which ear are they perceived most clearly? Give an explanation of this phenomenon.

15. Compound Tones.-Set in vibration a violin string fixed between two points. Touch the center of the string with a rod. Observe that the original fundamental tone is now obliterated, the lowest tone being an octave higher.

16. Observation of the Membrana Tympani in Man.-Fasten the reflector to your forehead, and direct the rays from a lantern into the right external auditory meatus of the subject. With your left hand pull the external ear backward and upward, and with your right hand insert the funnel-shaped tube, taking great care not to injure the skin of the meatus or the membrana tympani. Concentrate the light upon this membrane by moving the reflector either nearer to or farther away from

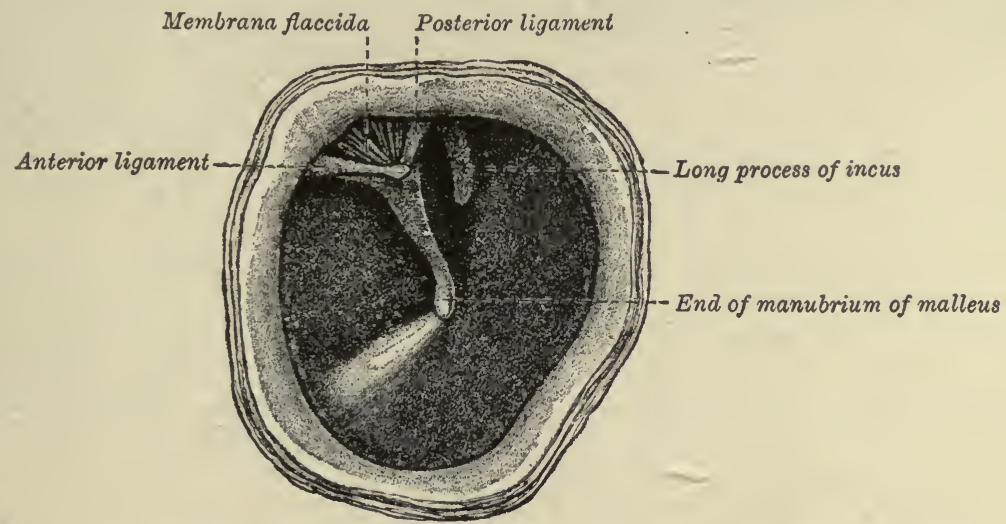

Fig. 101.-Membrana Tympani, as Seen with the Otoscope. (Heusman.)

the ear. Adjust the tube by tilting it, so that a view of the entire membrane may be had. The light should be placed about $60 \mathrm{~cm}$. from the reflector, and the reflector about $17 \mathrm{~cm}$. from the membrane.

Near the upper anterior border of the membrane will be seen the short process of the malleus, the handle of the malleus extending downward and backward from the short process. Locate the "umbo," which is the most retracted area of the membrane, and identify the "pars flaccida," the "pars tensa," and the "annulus cartilagineus."

17. Pressure in the Tympanum.-Close the mouth and nostrils. Attempt to inspire, and swallow. Note the peculiar sensation in the ears and the diminution in the acuity of hearing.

Close the mouth and nostrils. Attempt to expire, and swallow. A similar sensation is produced. Show how these acts affect the pressure in the tympanum and the vibratory quality of the ear drum.

18. Models of the Middle Ear.-Examine such models as may be available for illustrating the action of the ossicles. 
19. Observation of the Interior of the Larynx in Man.-Place a light near the side of the subject's head. Seat yourself in front of him,

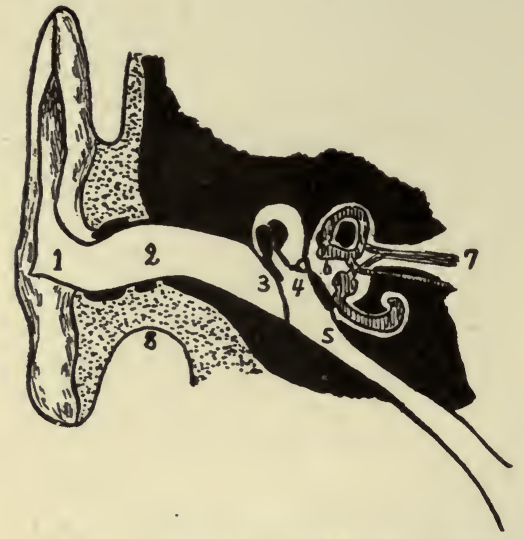

Fig. 102.-Diagrammatic Representation of the Different Parts of THE EAR.

1, Pinna; 2 , external auditory meatus; 3 , ear drum; 4, middle ear containing the ossicles; 5 , eustachian tube; 6 , vestibule of the internal ear; 7, auditory nerve; dividing into two branches, one of which innervates the cochlea, and the other the semicircular canals; 8 , parotid gland.

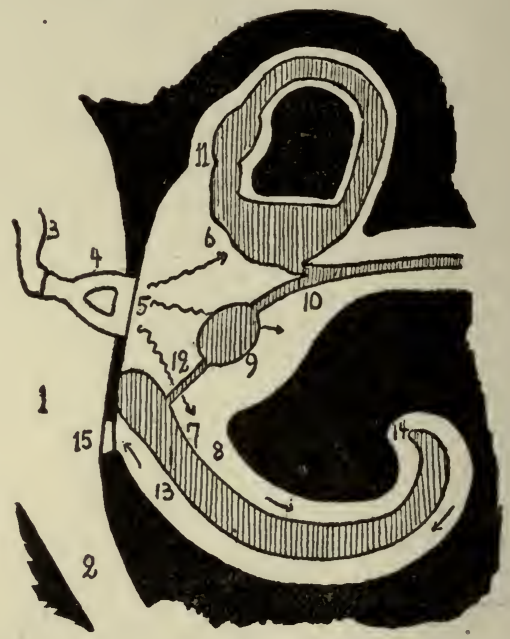

Fig. 103.-Diagrammatic View of the INTERNAL EAR.

1, Tympanic cavity; 2, eustachian tube; 3 , incus; 4 , stapes; 5 , vestibule of the internal ear (perilymph); 6, utricle; 7 , central canal of the cochlea; 8 , scala vestibuli; 9 , saccule; 10 , endolymphatic duct between saccule and utricle; 11, ampulla of semicircular canal; 12, canalis reuniens; 13 , scala tympani; 14 , helicotrema; 15 , fenestra ovalis.

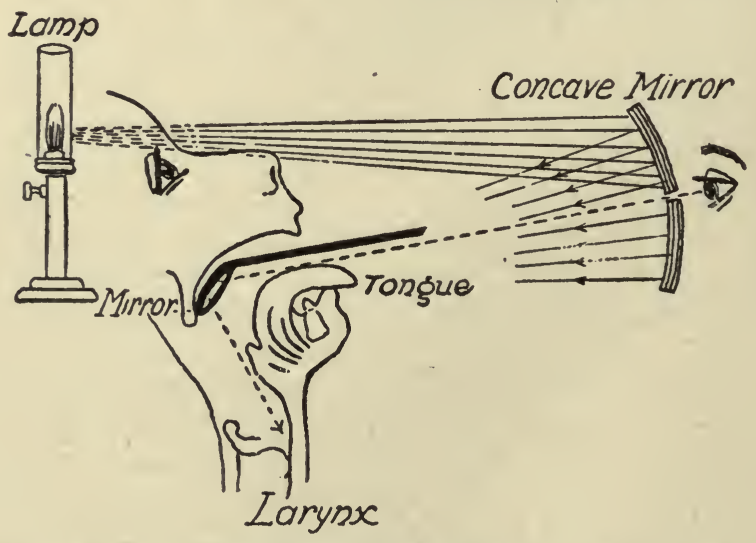

Fig. 104.-Diagram of Laryngoscope. (From Stewart's A Manual of Physiology, William Wood \& Co., Publishers.)

and direct him to incline his head slightly backward, to open his mouth, and to hold his tongue forward with the aid of a handkerchief. Illuminate the pharynx of the subject by means of a reflector fastened to your 
forehead. Slightly warm the laryngeal mirror, and pass it into the subject's mouth, carrying the mirror horizontally backward until its back touches the base of the uvula. Avoid pressure upon the uvula or contact with the other soft parts. Concentrate the light upon the mirror, and tilt the latter until a view is obtained of the interior of the larynx, remembering that the parts are seen inverted in the mirror.

Identify the dorsum of the tongue, the slightly yellowish epiglottis with its "cushion," and the glosso-epiglottidean folds of mucous membrane. Identify the white and shining "true" vocal cords, the pink or red "false" vocal cords, the aryepiglottidean folds, and the mucous membrane covering the arytenoid cartilages and cartilages of Santorini.

Observe the differences in the form of the glottis:

1. During quiet and forced respiration,

2. While the subject sings a low and a high note, and

3. While the subject sings the vowel sounds: A, E, I, O, U. 





\section{LESSON XXXVIII}

\section{THE SENSE ORGANS (Continued) \\ THE STATIC AND DYNAMIC SENSES}

1. Dissection of the Ear of the Dog-fish.-Remove the cartilage between the eyes. Identify the different parts of the brain. Proceed toward its hinder part, carefully removing the cartilage from within outward. Having reached one of the semicircular canals, remove its upper wall as far as possible. Identify its membranous canal with its ampulla. Follow this canal until it joins a membranous sac, the utricle. Carefully expose the other semicircular canals. Open one of the ampulla and identify the crista acustica, a transverse ridge carrying the sensory epithelium.

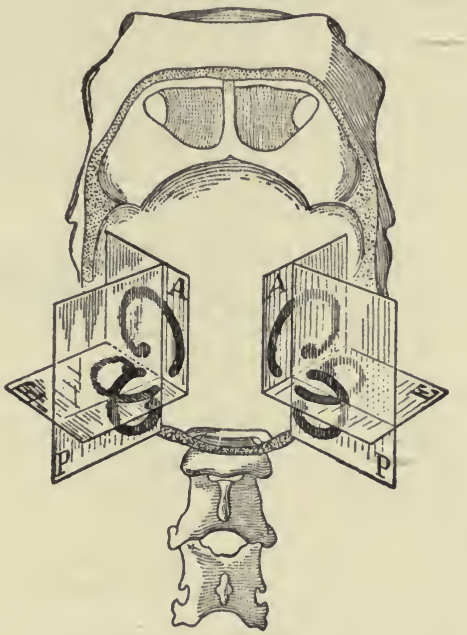

Fig. 105.-Position of the Three Semicircular Canals in the Skull of the Pigeon. (Ewald.)

2. The Position of the Semicircular Canals.-Procure a human skull in which the semicircular canals have been exposed, $i . e$. , the bone has been removed, leaving only the bony walls of the canals behind. Inside this shell lies the membranous canal containing endolymph and invested by perilymph.

Hold the skull in its proper position and indicate by a diagram the position of these canals. How many are there and what position do they occupy toward one another? What planes in space do they correspond to?

Diagrammatically represent the canals on the other side of the head, and compare their positions with those just sketched. Note that the canals are paired, comprising the following groups: the two horizontal 
ones, the right anterior and left posterior, and the left anterior and right posterior.

Identify the ampulla of each canal and determine its position. Tilt the skull in different directions, and state which canals are involved in any particular movement.

3. Model Illustrating the Action of the Semicircular Canals.Secure a model, such as is represented in Fig. 106. Rotate the circular glass tube containing water. Observe that the tube moves first and that at this time the water is still stationary The bristles, representing the hair processes of the cells lining the ampulla, are deviated against the direction of the rotation. Presently the water will move with practically the same velocity as the tube. The hair processes then extend straight into the fluid. On stopping the tube the water will continue to move onward, deviating the hair processes in the direction of its flow.

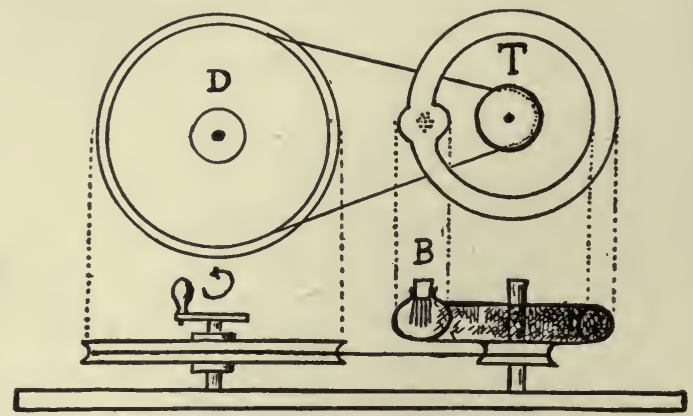

Fig. 106.-Diagrammatic Representation of a Model Illustrating the Deviation of the Hair Processes of the Ampulla.

$D$, Disk rotated by hand; $T$, circular glass tube filled with water; $B$, bulbular enlargement containing a long camel's-hair brush, vertically placed.

While a free movement of the endolymph is not possible, this schema illustrates at least the principle involved in the activation of the hair cells.

4. Acuity of the Dynamic Sense.- Sit upon a revolving chair. Close your eyes and lift the feet from the floor. Instruct the assistant to turn the chair a short distance. What canals are involved in this movement? Determine the number of degrees through which the rotation has taken place. Ascertain the least possible rotation which will impart a sensation of movement. How would you rate the acuity of this sense?

Bend your head forward. Repeat these tests. What canals are involved in this position? Is the sensitiveness of these canals toward this particular movement greater or slighter than that of the horizontal canals?

5. Rotation Effects in Mammals.-Place a rabbit in a long and narrow box which may be rotated around its vertical axis. Revolve the. box at a moderate speed about ten times around its axis. Immediately tilt the box so that the rabbit slides out upon the table. Observe 
the nystagmus, the change in the direction of the long axis of the rabbit, the change in the position of its head, and the compensating muscular movements made by it in order to retain its equilibrium. Do not repeat this experiment many times.

Stand erect and rotate a few times around the long axis of your body. Repeat this test with your eyes closed. Analyze the peculiar phenomena appearing when you cease rotating. In what direction do the walls move? How do you endeavor to counteract this impression? Since some persons are unusually sensitive to rotation, these experiments should be performed with some care. Do not rotate excessively until you have determined by a few rotations just how receptive you are.

Repeat the rotation around your vertical axis while you hold the head forward. Retain this position of the head at the end of the rotation. What canals are involved, and what is the character of the aftereffects?

Repeat the rotation around your vertical axis with the head bent forward. On ceasing to rotate, raise the head. Analyze the aftereffect.

6. Rotation Effects in the Frog.-Place a frog under a bell-jar upon a revolving chair. Turn the latter slowly, noting that the frog bends its head and body against the direction of the rotation.

Place a frog upon a somewhat roughened board. Tilt the board and observe that the frog bends its body, and chiefly its head, against the inclination. Carefully force the frog to move and make it move across the edge of the gradually raised board and down its opposite side.

7. Equilibrium a Combined Sense.-Close your eyes and try to stand on one leg for one minute. Open your eyes and repeat this test. Employ a tactile sensation in addition to the visual. Note that you can retain your equilibrium with greater ease if the sensations from the labyrinth are augmented by other sensations.

8. Railroad Nystagmus.-While riding in a street car observe that the eyes of the person seated opposite to you are first deviated laterally and are then quickly moved into a median position. This nystagmus is not of labyrinthine origin, because it may be made to cease by accommodation for a stationary object or by shielding the eyes.

9. Otolithic Cavity in the Frog.-Etherize a frog. Open its mouth widely and make an incision through the membrane covering its roof on the median side of the orifice of the Eustachian tube. Remove the surface layer of the bone of this region, thereby exposing a white otolithic mass. Thoroughly destroy this mass. Let this animal rest for some time, and then observe the position of its head and limbs. Rotate this frog and look for compensating movements. Does this frog possess a normal power of locomotion? How do its swimming movements compare with those of a normal frog?

Destroy the otolithic cavity on the opposite side, and repeat the observations just made. Compare. 





\section{LESSON XXXIX}

\section{THE SENSE ORGANS (Continued)}

\section{VISION}

1. Dissection of the Eye.-Procure an ox eye. Having identified the lids, conjunctiva, and different structures attached to the eyeball, isolate the optic nerve and cut away the muscles and fatty tissue. Open the anterior chamber of the eye by a transverse incision through the cornea. Study the physical characteristics of the aqueous humor. Remove the cornea and examine the iris and neighboring parts. By exerting a gentle pressure upon the outer coat of the eyeball force the

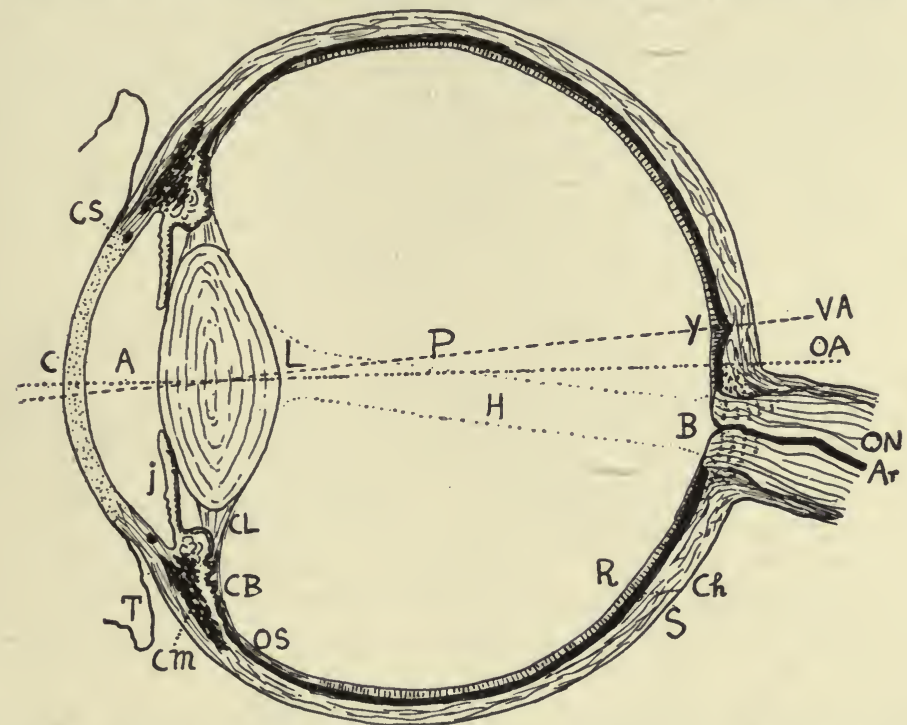

Fig. 107.-Diagram of a Horizontal Section Through the Human Eye.

$C$, Cornea; $A$, anterior cavity; $P$, posterior cavity; $L$, lens; $J$, iris; $T$, conjunctival sac; $C L$, ciliary ligament; $C B$, ciliary body; $C M$, ciliary muscle; $O S$, ora serrata; $C S$, canal of Schlemm; $R$, retina; $C h$, choroid; $S$, sclera; $O N$, optic nerve; $A$, retinal artery; $B$, blind spot; $Y$, yellow spot; $O A$, optical axis; $V A$, visual axis; $H$, hyaloid canal.

lens through the pupillary orifice. Examine the lens, noting the degree of convexity of its anterior and posterior surfaces. Hold it over print. Place it under water. Can it be readily seen now? Explain. Open the posterior chamber widely, noting the differences in the color of the fundus. Explain. Identify the optic papilla and obtain an idea regarding the location of the yellow spot. Note the characteristics of the vitreous humor. Examine histologic preparations of the cornea, iris, lens, and retina. 
2. Formation of the Image Upon the Retina.-Place a fresh ox eye in a watch-glass. Make a small square opening in its upper wall directly behind the ciliary body. Direct the pupil of this eye toward an incandescent lamp (optical lantern), the rays of which have been rendered parallel by the interposition of projection lenses. Vary the distance between the light and the eye until the rays have been made to intersect sharply upon the retina. Place a diaphragm with an upright arrow in front of the projection lens. Note the condition of the image. By means of a diagram show why the image must be inverted. Move the incandescent lamp (optical lantern) slightly in different directions and observe the direction of the movement of the image.

Endeavor to thin the eyeball in the region of its posterior pole by removing the sclera. This means is frequently resorted to in order to obtain a clearer image. It illustrates the manner of focalizing an object upon the ground glass of a photographic camera.

3. Changes in the Size of the Pupil.-Light Reflex.-Observe the changes in the size of the pupil in a subject who alternately accommodates for a window and a darkened wall. Cover the eye of the subject with the flat of your hand for a few moments. Remove the hand. Note the constriction of the pupil. Again close one eye with your hand and observe the size of the pupil of the other eye. Suddenly remove the hand. Note that the pupil of the other eye also constricts.

The cornea acts as a planoconvex glass and gives a larger size to the pupil than it actually possesses. To show this take an ox eye, the cornea of which has been removed. Place a watch-glass in front of it. The pupil immediately appears larger.

In order to show the movements of the iris in your own eye proceed as follows: With your right eye look at a uniform white surface through a pinhole in a card, preferably at the white shade of a reading lamp. Close your left eye. Obtain a concept of the size of the circular visual field. Open the left eye. The field becomes smaller and brighter, owing to the constriction of the pupil. Again close the left eye. The field gradually enlarges and is slightly dulled, owing to the dilatation of the pupil.

Accommodation Reflex.-Observe the changes in the size of the pupil in a subject who alternately looks at objects near to and far away from his eye.

4. Changes in the Shape of the Lens.-Look at the eye of the subject from the side, observing the position of the iris when accommodated for a far object. Ask the subject to accommodate for a near object. Note that the iris is forced forward into the aqueous chamber, owing to the fact that the lens now becomes more convex.

Insert. an ordinary watch-glass in a tube of black paper, its convexity being turned outward. A few centimeters behind it adjust a biconvex lens. Hold the tube toward a candle, and note the three images reflected from this system, viz., one from the watch-glass (cornea), one from the anterior surface of the lens, and one from the pos- 
terior surface of the lens. The first two images are upright and the third inverted.

In a thoroughly darkened room place your eyes about $25 \mathrm{~cm}$. in front and to the left of the right eye of the observed person. With your left hand hold a large cardboard directly beside the right side of
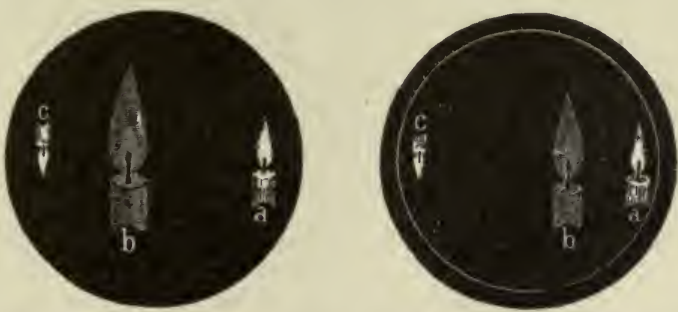

Fig. 108.-Reflected Images of a Candle Flame as Seen in the Pupil of an Eye at Rest and Accommodated for Near ObJects. (Williams.)

your head. With your right hand hold a lighted candle somewhat to the right of the cardboard, $i$. e., to the right of the visual axis of the eye of the observed person. Vary the position of the candle and approach the eye of the subject until you can clearly make out three images, viz., one from the cornea, one from the anterior surface of the lens, and

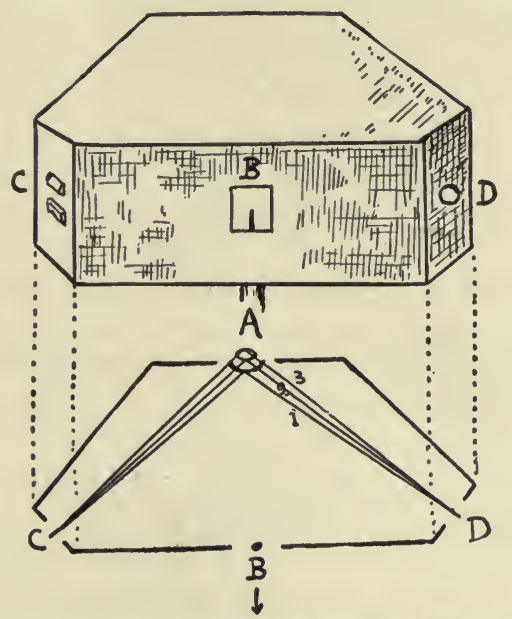

Fig. 109.-Diagram Illustrating Course of the Rays Through the Phacoscope.

$A$, Observed eye; $B$, opening allowing accommodation for near and far objects; $C$, source of light; $D$, observer's eye; 1 , images from cornea; 2 , anterior surface of lens; 3 , posterior surface of lens.

one from the posterior surface of the lens. The gaze of the subject should at this time be directed straight ahead upon a distant object. Note the position and appearance of the images. Ask the subject to accommodate for a near object, but without changing his visual line. What changes do you note in the position and shape of the images? 
Draw conclusions regarding the relative importance in accommodation of these refractive surfaces.

Repeat these observations with the help of the phacoscope (Helmholtz).

Annotation.-The phacoscope consists of a roughly triangular box. The observer's eye is placed in the aperture at $A$ and focalizes a far object through aperture $B$. Orifice $C$ contains two prisms, through which light is reflected upon the eye of the observed person. The observer notes the images through the orifice $D$. The observed person then accommodates for the pin situated in aperture $B$ (Fig. 109).

Since the image from the cornea does not change its position or shape, the cornea does not alter its refractive power. The inverted image from the posterior surface of the lens undergoes a very slight change, showing that this refracting surface remains practically unchanged. Contrariwise, the image from the anterior surface of the lens becomes more rounded and moves toward the corneal image. This change proves that this refracting surface suffers the principal change in accommodation.

5. Wabbling of the Lens.-Gaze upon a light wall or ceiling. Do you notice black dots traversing the visual field (muscæ volitantes)? If you do, quickly accommodate for a near object and note that the spots execute a jerky lateral movement. This fact indicates that accommodation relieves the tension under which the lens is ordinarily held and allows it to deviate somewhat from its optical axis. This phenomenon constitutes the so-called wabbling of the lens.

6. Formation of the Retinal Image.-Accommodate for a light object situated about $6 \mathrm{~m}$. from the eye. Hold the index-finger of your right hand in the visual axis of this eye. Draw a diagram to show why the finger appears indistinct. Repeat this experiment, but accommodate for the finger. Draw a diagram showing why the far object is not clear.

Look at a window through wire netting held about $25 \mathrm{~cm}$. in front of the eyes. Later on accommodate for the netting. Explain.

7. Scheiner's Experiment.-Make two small holes in a card at a distance of $4 \mathrm{~mm}$. from one another. Close one eye and hold the openings in front of the pupil of the other eye. Accommodate for a pin held about $18 \mathrm{~cm}$. in front of the eye. The pin is seen single. Now, accommodate for a pin held at a distance of $60 \mathrm{~cm}$. from the eye. The far pin is seen single and the near pin double. Close the left opening in the card and observe which image disappears when accommodating for the far pin and which when accommodating for the near pin. Draw diagrams showing the course of the rays, and explain the peculiar blocking of the images following the closure of one or the other of the openings in the card.

The psychic element in vision is also clearly betrayed by the following experiment: With the left hand hold a card bearing a pinhole about 3 or $4 \mathrm{~cm}$. in front of the eye. The other eye should be closed at this time. With the right hand bring the head of a pin from below into the field of vision, adjusting the pin as close as possible to the pupil. Note 
that the pin appears to enter the visual field from above. Draw a diagram to illustrate the course of the rays and explain this peculiar phenomenon.

8. The Emmetropic Eye.-Adjust the optical box at a distance of about $15 \mathrm{~cm}$. from the optical lantern. Obtain parallel rays by placing the tin tube containing the projection lenses in front of the incandescent light. Lessen this bundle of light by a diaphragm, bearing the outlines

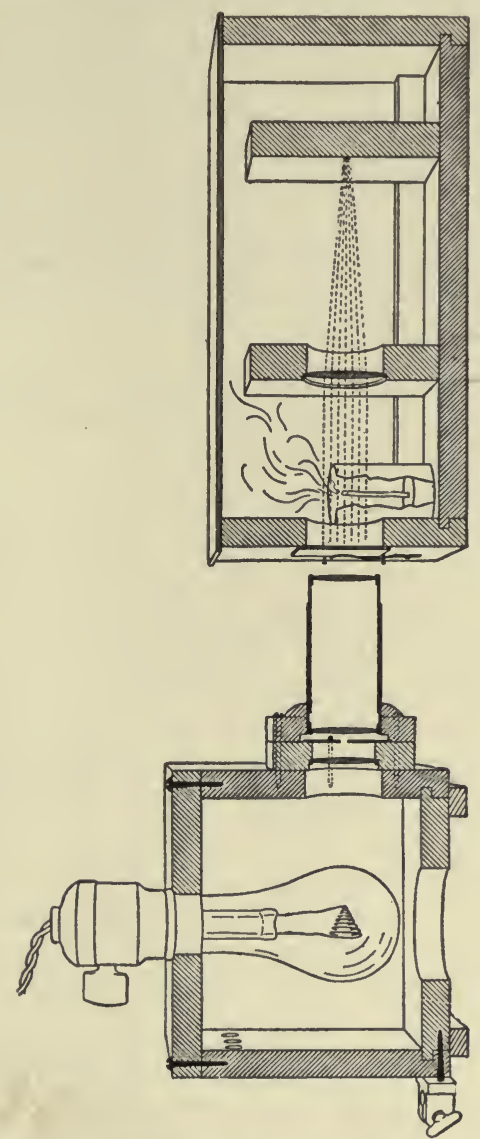

Fig. 110.-Optical Lantern and Box. (Harvard Apparatus Co.)

of the letter $L$. Close the round opening in the optical box by a flat piece of window glass (cornea). In the holder directly behind this opening place a convex lens of $10 \mathrm{~cm}$. focal distance (10 diopters). Arrange the solid black screen (retina) near the center of the optical box. Close the box with a large plate of clean glass and burn a small amount of Japanese incense in the box. Move the optical box as well as the screen (retina) until a perfectly clear image of object $L$ is obtained upon the latter. 
9. Circles of Dispersion.-Move the screen farther forward and then farther backward. Note that the rays now strike the retina widely apart, $i$. e., they form a dispersion circle upon this receptor.

The same end may be attained by moving the lantern either farther away or nearer to the optical box (lens). Draw diagrams showing the course of the rays under these two conditions.

10. Near Point.-Again adjust the screen (retina) in such a way that the rays of light emitted by object $L$ are brought to a sharp intersecting point (emmetropia). With a ruler determine the distance between object $L$ and the lens of the optical box. This distance constitutes the anterior focal distance of this lens and indicates the position of its near point.

Move the lantern closer to the optical box. Observe that the rays immediately pass into dispersion and finally leave the posterior surface of the lens very divergently, illuminating the screen widely. A precise focal point cannot be obtained if the object is situated inside the near point.

11. The Ametropic Eye. Hypermetropia. Myopia.-Construct an eye that is too short. To accomplish this end move the screen $2.5 \mathrm{~cm}$. in front of the position occupied by it when the eye was emmetropic. This adjustment simulates the condition of hypermetropia or fnr-sightedness. Theoretically speaking, the focal point of these rays lies behind the screen. In order to render this eye emmetropic place a convex lens in front of the aperture in the box (cornea), its strength being just sufficient to converge the rays so that they intersect upon the screen. A lens of $2 \mathrm{D}$. should accomplish this end. If a number of different lenses are available, other degrees of hypermetropia may be established.

Construct an eye that is too long. To accomplish this end move the screen a distance of $2.5 \mathrm{~cm}$. behind the position occupied by it in the emmetropic eye. Note that the intersecting point of the rays now lies in front of the screen (vitreous humor) and that the rays then diverge and strike the screen in dispersion. This condition is known as myopia or near-sightedness. In order to correct this condition interpose a concave lens in front of the optical box (cornea). The entering rays are thereby rendered more divergent, so that they are focalized exactly upon the retina. A -2 lens $(-2 \mathrm{D}$.) will accomplish this end, provided the degree of myopia which has been established is not greater than specified.

Draw diagrams to show the manner of refraction in hypermetropic and myopic eyes, and also indicate how these errors may be corrected.

At the close of this exercise replace every lens in its proper paper envelope. 




\section{LESSON XI \\ THE SENSE ORGANS (Continued)}

VISION

1. The Ametropic Eye. Astigmatism.-Adjust the optical lantern and box to form an emmetropic eye. Insert the diaphragm with the $2 \mathrm{~mm}$. aperture in the opening of the optical lantern. Place a beaker with water directly in front of the cornea. Note that the image now simulates a vertical line. Close the top of the beaker by means of a small piece of cardboard. Hold the beaker between your thumb and index-finger and place it horizontally in front of the cornea. Note that the image now simulates a horizontal line. Draw a diagram to show the course of the refracted parallel rays.

Repeat the preceding tests by holding a cylindric lens in front of the cornea, first with its greatest curvature adjusted in the vertical direction and then adjusted in the horizontal direction. By this means may be imitated the conditions of "with-the-rule" and "against-therule" reguiar astigmatism. It will be remembered that the astigmatism which the physician is usually called upon to correct is due to a faulty curvature of the cornea. In each case move the screen farther forward and backward and note the changes in the shape of the image. Explain these changes by means of a diagram.

By means of a cylindric lens establish the condition of "with-therule" astigmatism. Move the screen so as to obtain as distinct an image as possible. Correct this condition by interposing in front of this cylindric lens another one of the same refractive power, but adjusted in such a way that its greatest curvature comes to lie in the horizontal plane of the cornea.

Establish and correct the condition of "against-the-rule" astigmatism in the same manner.

Explain the fact that in the absence of a faulty curvature of the cornea, all round luminous objects, such as lamps and stars, do not appear round, but as radiate figures.

2. Chromatic Aberration.--Let the rays from the lantern emerge parallel through the $2 \mathrm{~mm}$. aperture. Hold a lens of $10 \mathrm{D}$. about 15 $\mathrm{cm}$. in front of the.lantern, $i$. e., at a distance greater than the focal distance of this lens. Place a white sheet of paper in the path of the converging bundle of light at a distance of about $15 \mathrm{~cm}$. from the lens. Note the colors around the margin of the image. Correct this aberration by covering the edges of the lens with a circle cut out of cardboard. How is chromatic aberration prevented in our eye?

Make a pinhole in a card and place it upon cobalt glass. Close one eye and with the other gaze at a gas flame through the pinhole. The 
cobalt glass permits only the red and violet rays to pass. In accommodating for the red rays a violet halo is obtained and on accommodating for the violet rays, a reddish halo.

On a black background place a strip of red paper and one of blue paper. The red appears nearer than the blue. Since the red rays are less refrangible, a greater effort at accommodation is required to focalize them. This gives rise to an erroneous judgment of distance.

Place a heart colored red upon a bright blue sheet of paper. In a room lighted only by a candle hold the heart below the level of the eyes and move it gently from side to side. The red heart will then appear to flutter over the blue background.

3. Spheric Aberration.-Construct an emmetropic eye. Note that while the focal point upon the screen seems perfectly sharp, a close observation shows that this "point" is really drawn out backward. This distortion is due to the development of caustics, $i$. e., the rays of light traversing the marginal zone of the lens are focalized somewhat behind those traversing its central area. Correct this defect by covering the peripheral sphere of the lens with a circle cut out of paper. How is spheric aberration prevented in our eye?

4. Mechanical Stimulation of the Retina. Phosphenes.- Shut one eye and turn it inward. With the point of a pencil press gently upon the outer surface of the upper eyelid. To what part of the field of vision is the yellowish circular image referred. Explain.

Place a light in front of the eyes. Close them and move them quickly from side to side. When the eyes reach an extreme position, observe the rapidly disappearing bluish spot surrounded by a yellow halo. Obviously, this movement stimulates the retina around the optic papilla in a mechanical way. Note that mechanical stimuli are inadequate stimuli. While they produce visual sensations, they cannot give visual concepts. Moreover, all retinal impressions are always projected into the opposite visual field, $i . e$., into that part of the field with which that particular area of the retina is in functional relation.

5. The Field of Vision.-Fasten a sheet of white paper $60 \mathrm{~cm}$. square upon a piece of pasteboard and make a small cross mark about $30 \mathrm{~cm}$. to the right of the left margin of the paper. Let the subject rest his chin upon an iron support, and adjust the paper in such a way that his right eye is in a direct line with the cross mark. Move the vertically placed paper close enough so that he cannot look beyond its edges. Let the experimenter fasten a small piece of white paper to a straw, move it horizontally inward from without, and mark on the white sheet the point at which this object becomes clearly perceptible to the person. Determine in this way also the boundaries of the visual field of this eye in the vertical direction, and in two or three intermediate directions. Outline the inner side of the field in a corresponding manner. Connect the different pencil marks to form a continuous line. The visual field of the left eye may then be mapped out in a similar 
way. The sheet of paper, however, must then be adjusted in front of this eye and bear a cross mark near its right margin.

If a perimeter is available, the subject should be comfortably seated at a table with his chin resting upon the support. Either the right or the left eye should then be focalized steadily upon the white dot in the center of the semicircle. The metal arch bears a sliding path in which is moved the object, generally a small white square, from without inward, its movement being indicated in degrees upon a scale inscribed upon the arch. The point at which the object becomes first visible

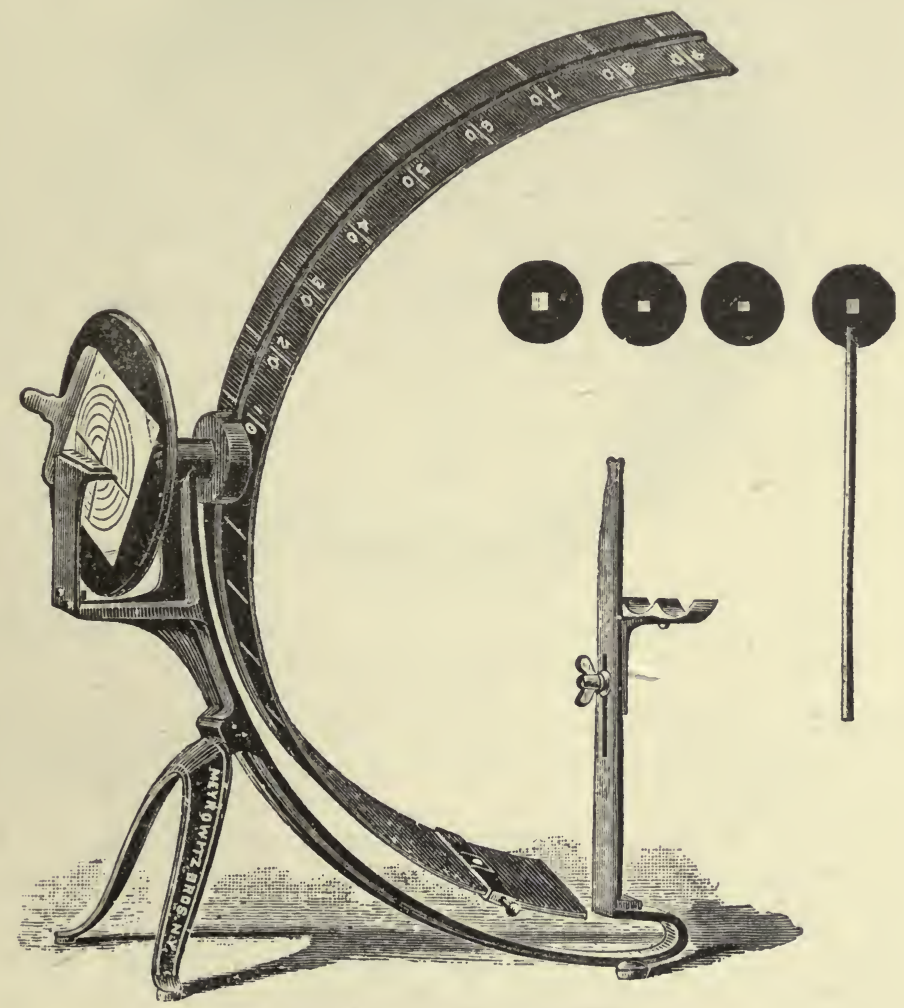

Fig. 111.-The Perimeter.

is then indicated upon a small piece of paper upon which different meridians have been drawn out to correspond to those of the metal arch.

Study the general outline of the visual field of each eye. Why is it not round, but oval, with its more pointed area toward the nasal side? Do you note any irregularities in its outline which cannot be ascribed to a faulty technic? Enumerate the causes which might be held responsible for such restrictions of the visual field.

6. The Fields for Colored Objects.-Proceed as described in paragraph 5 , but insert red, blue, and green squares in the holder upon the 
metal arch. While the subject gazes fixedly at the mark in the center of the arch, move these colors interchangingly from without inward along the different meridians of the eye. As soon as the subject perceives the color clearly, indicate this point in degrees upon the smaller chart. Eventually, connect all the points of the same color with one another.

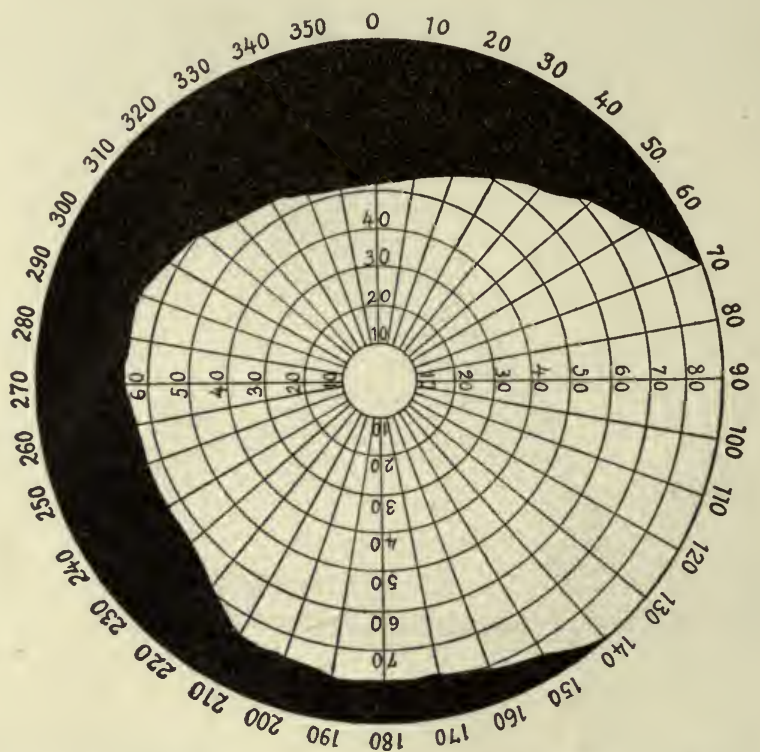

Fig. 112.-Perimeter Chart to Show the Field of Visiun for a Right Eye When KePt in a Fixed Position.

While the results differ materially with the saturation of the different colors, most generally the red field is the largest, then follows the blue, and lastly the green.

7. Entoptic Phenomena Produced by the Tears.-Evoke a mild hypersecretion of lacrimæ by holding a cut onion at some distance below the eye or imitate this condition by moistening the eyelids with fresh water. Close the eyelids somewhat, so as to deepen the layer of lacrimæ upon the central area of the cornea. What effect has this upon refraction and the formation of the retinal image? Draw a diagram to show how refraction is affected under water. 




\section{LESSON XLI \\ THE SENSE ORGANS (Continued) \\ VISION}

1. The Near and Far Points of the Eye.-Hold a pin $50 \mathrm{~cm}$. from your eyes and move it slowly toward them until a point has been reached beyond which this object no longer produces a clear image. Measure the distance of this point from your eyes and determine whether, in accordance with your age, it is situated at a normal distance. Deter-

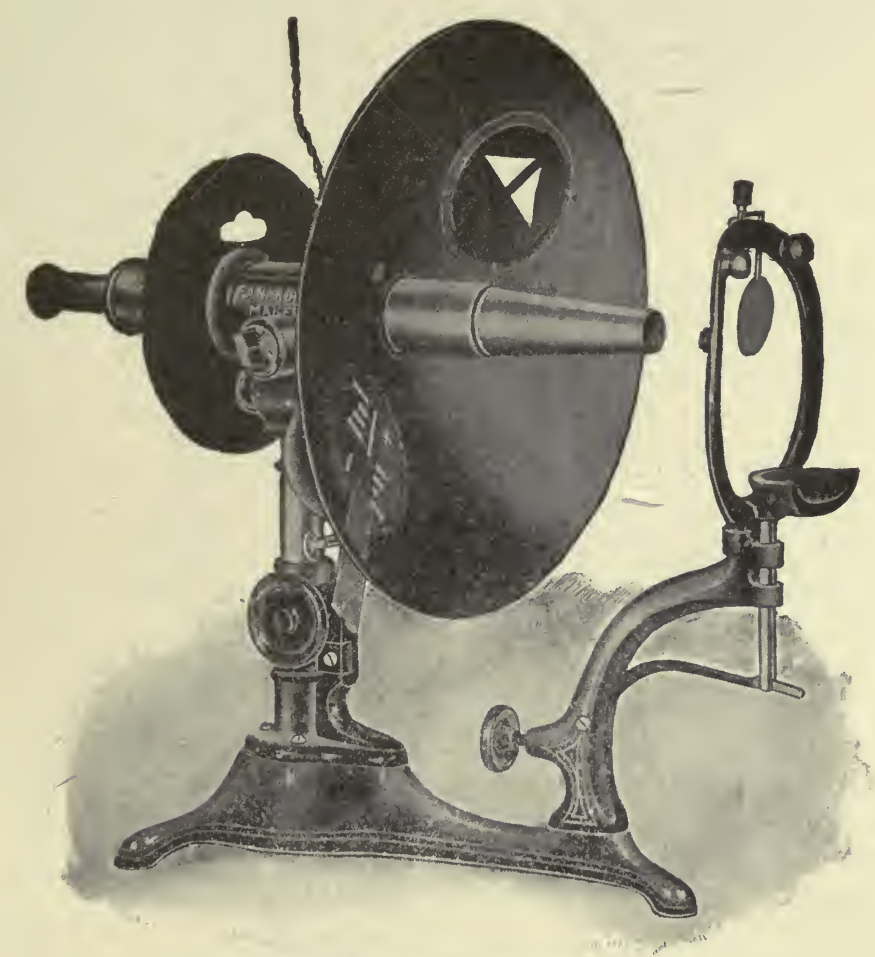

Fig. 113.-Ophthalmometer. (Hardy.)

mine the near point for each eye. In the emmetropic eye the far point lies at the horizon.

Select a number of students with abnormal vision and determine the near and far points in each. Ascertain whether they are far-sighted or near-sighted. 
2. Detection of Astigmatism.-Determine the near point for a pin held vertically and one held horizontally. Note whether the distances correspond. If not, what are your conclusions.

Draw two lines $5 \mathrm{~cm}$. in length and intersecting at right angles. Each right angle again bisect and each oblique angle again by other lines, until a radiate figure is obtained. Interpose this figure at the near point of accommodation. Note whether any of these lines appear blurred. Explain.

3. Measurement of Astigmatism.-Carefully study the construction of the ophthalmometer (Helmholtz), and discuss the principle involved in this method of detecting and measuring astigmatism.

4. Visual Angle.-The visual angle is the angle formed by the lines drawn from the two extremities of an object through the nodal point of the eye (optical center of the lens). It is situated $7 \mathrm{~mm}$. behind the cornea and $15 \mathrm{~mm}$. in front of the retina. Since these lines traverse the nodal point unrefracted, the size of the retinal image may be obtained from this projection. Draw a diagram to show that, in order to subtend at the same angle, objects must be made increasingly larger, the farther they are removed from the retina. Thus, the letter $A$ seen clearly at $6 \mathrm{~m}$., would have to be ten times as large at $60 \mathrm{~m}$. in order to be seen equally well. At a distance of $1 \mathrm{~m}$. this letter should be $1 \mathrm{~cm}$. in height in order to be seen clearly by the emmetropic eye.

5. Snellen's Test Types.-Determine the normal acuity of vision by distinguishing different letters subtending at an angle of 5 degrees. If the distance indicated can be exceeded or cannot be reached, the acuity of vision may be expressed as $=\frac{D}{d}$. In this formula D stands for the given distanee; at which the angle of 5 degrees is subtended, and $d$ for the distance at which the letters can be recognized.

Equip the ametropic person (myopic or hypermetropic) with lenses of different refractive power until able to recognize these letters clearly.

6. Ophthalmoscopic Examination of the Emmetropic Artificial Eye by the Direct Method.-Remove the projection-lens from the optical lantern. Adjust the emmetropic artificial eye for far vision at zero, and place it to the left of the lantern and on a level with your own eye. With the right hand hold the ophthalmoscope close to your right eye and about $30 \mathrm{~cm}$. from the artificial eye. Make the visual axis of your right eye coincide with that of the artificial eye by keeping your head erect. Look through the opening in the mirror and throw the light into the pupil of the artificial eye. Accommodate your own eye for an imaginary object placed at some distance precisely behind the artificial eye. Gradually move your head toward the artificial eye until the mirror lies in the anterior principal focus of the latter, $i . e .$, about $5 \mathrm{~cm}$. in front of the cornea of the artificial eye.

When the fundus of the artificial eye has become visible, find the optic disk. Draw a diagram showing the course of the rays of light.

In case the observer is myopic or hypermetropic, he must first cor- 
rect his error in refraction by placing a suitable lens behind the opening in the mirror.

\section{Ophthalmoscopic Examination of the Ametropic Artificial Eye by} the Direct Method.-Render the artificial eye ametropic by pushing its two parts closer together or by separating them more widely. Examine its retina with the ophthalmoscope held at a distance of from 30 to $50 \mathrm{~cm}$. in front of it. When the details of the fundus have become visible, move your head together with the ophthalmoscope from side to side. In the hypermetropic eye the retinal vessels will appear
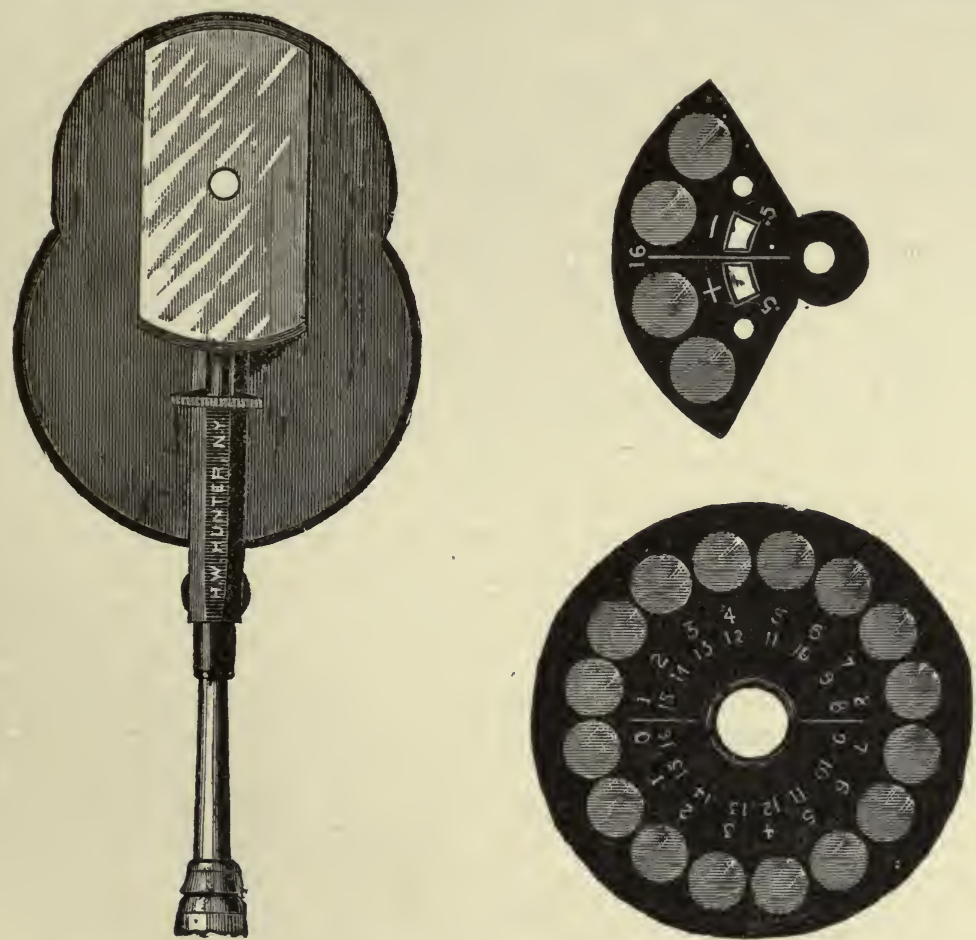

Fig. 114.-Loring's Ophthalmoscope, with Tiliting Mirror, Complete Disk of LENSES FROM -1 TO -8 AND 0 TO +7 , AND SUPPLEMENTAL QUADRANT ConTAINING \pm 0.5 AND $=16 \mathrm{D}$. This AfFords 66 Glasses or Combinations from +23 to $-24 \mathrm{D}$.

to move in the same direction, and, in the myopic eye, in the opposite direction. Having in this way determined whether the eye is myopic or hypermetropic, measure the degree of this ametropia in the following manner:

Place the ophthalmoscope in the anterior focal plane of the artificial eye, $i$. e., $5 \mathrm{~cm}$. in front of its cornea. Illuminate the pupil and accommodate your own eye for a distant point. If the artificial eye is myopic, place behind the opening in the mirror concave lenses of different strengths, until the lens has been found which renders a certain part of the optic disk-for example, a peripheral blood-vessel-perfectly 
clear. If the artificial eye is hypermetropic, use convex lenses instead of concave. The focal power of the lens which is needed in order to be able to see the fundus of the eye of the subject clearly, is the lens needed to overcome the ametropia of this eye. In prescribing glasses for patients the strength of the lens is somewhat exaggerated, $i$. e., we prescribe in hypermetropia the strongest convex lens with which the observer is still able to see the details of the fundus, and in myopia the weakest concave lens. Why? Draw diagrams to show the course of the rays emitted by myopic and hypermetropic eyes. Also indicate how these conditions may be corrected by the interposition of suitable lenses.

8. Ophthalmoscopic Examination of the Artificial Eye by the Indirect Method.-Place a convex lens of 5 diopters behind the opening in the mirror of the ophthalmoscope, and hold the latter about $30 \mathrm{~cm}$. in front of the artificial eye. Interpose at a distance of $5 \mathrm{~cm}$. from the cornea of the artificial eye a +20 lens, held between the thumb and forefinger of the left hand. The rays which issue from the artificial eye are focalized in space after they have passed through the +20 lens. This image you study with the aid of the +5 lens. Draw a diagram to show the course of the rays emitted by this eye.

9. Skiascopy or the Shadow Test.-Hold the plane mirror of the ophthalmoscope in front of your eye and at a distance of $1 \mathrm{~m}$. from the emmetropic artificial eye. Throw a beam of light upon this eye. That portion of it which falls upon the pupil is condensed on the retina and forms here an area of light which moves in accordance with the movement of the mirror. Consequently, the same condition is obtained here as when light is reflected against a wall by means of a mirror. Draw a diagram to show the real movement of the light upon the retina. note that its movement, as seen through the pupil, is the same as that of the light on the face.

Render the artificial eye myopic by drawing its halves farther apart. Place the mirror at a distance of $1 \mathrm{~m}$. from the eye and tilt it gently. Note that the pupillary reflection now moves in a direction opposite to that of the mirror. Move closer to the eye until a point has been reached at which it is impossible to tell which way the light moves. Move still closer to the eye and observe that the light now moves with the rotation of the mirror. It is to be remembered that the myopic eye converges the rays leaving it. The point in space at which they intersect is the point of reversal. Obviously, distally to this point the light must move against, and inside this point, with the rotation of the mirror. Hence, skiascopy is simply an accurate method of determining this point of reversal. Draw a diagram illustrating the reflection in myopia.

Render the artificial eye hypermetropic by bringing its halves closer together. Place your eye at a distance of $1 \mathrm{~m}$. from the artificial eye. Move back and forth and note that the movement of the pupillary light is with the rotation of the mirror, no matter what position you occupy. This is due to the fact that the hypermetropic eye does not form a 
point of reversal because it emits divergent rays. Now interpose a convex lens in front of the artificial eye, thereby converging these rays and producing an artificial point of reversal and, so to speak, an artificial myopia. This lens accomplishes two things, namely, it neutralizes the divergence of the rays, and secondly, converges them sufficiently to intersect. In order to obtain the degree of hypermetropia existing in this eye, determine the point of reversal as in normal myopia. Subtract the degree of myopia from the total strength of the lens. The remainder of the focal strength of the lens is the strength which is required to overcome the hypermetropia. Draw a diagram showing the reflection in the hypermetropic eye.

Select a number of students who are either near-sighted or farsighted and determine the degree of ametropia in each. For example, if the erect movement is obtained as close as $55 \mathrm{~cm}$. from the eye and the reversal as near as $80 \mathrm{~cm}$., the point of reversal lies at a distance of about $67 \mathrm{~cm}$. The myopia equals $1.50 \mathrm{D}$. Again, supposing that the movement of the light in the pupil is found to be with the mirror at all distances, then interpose a $5 \mathrm{D}$. (convex) lens. - If the point of reversal is now at a distance of $1 \mathrm{~m} ., 4 \mathrm{D}$. have been used to neutralize the divergency and $1 \mathrm{D}$. to render the rays convergent. Consequently, the degree of hypermetropia equals $4 \mathrm{D}$. 



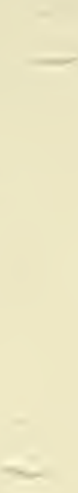

D 



\section{THE SENSE ORGANS (Concluded)}

VISION

1. The Blind Spot.-Make a small cross mark upon a white strip of paper. About $8 \mathrm{~cm}$. to the right of it make a black dot of the size of a large pea. Close your left eye and with your right eye gaze fixedly at the cross lines, allowing the dot to lie in the outer visual field. Bring the card closer to the eye until the dot disappears completely. Move the card still closer; the dot reappears. Draw a diagram explaining this defect in the visual field. What position does the yellow spot occupy in relation to the blind spot, and how is this disturbance overcome in binocular vision.

With your right eye gaze at an object placed at a distance of $6 \mathrm{~m}$. from you. Adjust the position of a fellow student in such a way that his head disappears completely.

Make a small cross upon a white strip of paper. On each side of it and about $4 \mathrm{~cm}$. from it make two black dots. Hold your left hand with its inner margin against the bridge of your nose. With your right hand hold the above figure in front of your eyes, and while steadily fixing the cross move the paper to and fro until you reach the distance at which both dots disappear.

2. The Contours of the Blind Spot.-Place the chin of the subject upon a support and adjust a sheet of white paper about $50 \mathrm{~cm}$. vertically in front of him. Ask him to gaze with his right eye at a small black dot upon the paper. Fasten a pin with a large black head upon a straw, and move this pin from without inward along the horizontal meridian of the eye. Indicate upon the paper the moments when the head of the pin disappears and reappears. Draw a vertical line about midway between these two points and also several oblique lines. Indicate upon all these lines the moments when the pin disappears and reappears. Connect these points to obtain a continuous line. Note that the field is irregularly oval in its outline, owing to the fact that the blood-vessels emerging from the optic disk are also insensitive to the light rays.

3. The Yellow Spot.-Having rested the eye for a minute or two, look through a flat bottle containing a fairly strong solution of chrome alum. It is best to hold the bottle against a sheet of white paper. Since the pigment of the yellow spot absorbs the blue and green rays and transmits the others, the predominant tinge imparted to the area corresponding to the macula lutea will be red (purple).

4. The Retinal Blood-vessels.-While the subject turns his eyes laterally upon a dark wall, concentrate a beam of light from the optical lantern upon the exposed sclerotic coat directly behind the region of 
the ciliary body. Give the lens used to concentrate the light a gentle rocking motion. The visual field will then appear to the subject as reddish yellow, through which dark figures are passing. The latter assume the character of a network, in agreement with the branching blood-vessels (Purkinje's figures). By this means shadows of the blood-vessels are cast upon regions of the retina not ordinarily exposed to them. It is essential, however, that these shadows move, because the retina quickly adapts itself to continued stimulation.

Make a pinhole in a card and hold it directly in front of one eye, the other being closed. Direct the visual axis upon a bright and evenly illuminated sheet of white paper placed in front of a lamp. Move the card from side to side. Vertically running figures will be apparent. Move the card up and down. Horizontal vessels will now be in evidence.

Gaze at a white cloud through a thick piece of blue glass. Many bright points followed by shadows will be seen to traverse the visual field in a constant procession. The latter, in all probability, represent the red blood-corpuscles.

5. Direct and Indirect Vision.-Draw a figure upon a white sheet of paper, consisting of one central dot surrounded by six others at a slight distance from it. With your right eye fixate a small object at a distance of $50 \mathrm{~cm}$. from you. Bring the aforesaid figure slowly into the line of vision. Note that the individual dots coalesce at some distance from the latter. They are severally in evidence only when the figure is brought into the visual line. Obviously, at this time, the rays of light emitted by the figure are focalized in the fovea, $i$. e., in the region of the greatest acuity of vision. When'made to fall upon outlying districts of the retina the rays cannot form a precise image.

Gaze at an object in a brightly illuminated room. Note that the object is unconsciously brought into direct line with the fovea. Darken the room. Note that at first your vision is very poor, but gradually adapts itself to the low intensity of light. Also observe that you now endeavor to focalize objects by bringing them upon the outer zones of the retinæ.

Focalize a single word upon a page of print. Note that only the word looked at is perfectly clear, while those farther away from it in any direction are not clear.

Draw radiate lines upon the blackboard and equip each with cross marks at distances of 5 degrees from one another. Fix any one of the cross marks and note that the others are at this time not perfectly clear.

6. Associated Movements of the Eyes.-Close one eye and place the tip of your index-finger upon the upper eyelid. Note that the focalizing of an object with the other eye invariably produces a co-ordinated movement in the closed eye.

7. Positive After-images.-Having rested the eyes for a short time, look suddenly at a lighted incandescent lamp and shut the eyes again after a distinct image of the lamp has been formed. Note the aftereffect. 
Close the eyes for a few seconds. Look at a gas flame in which some common salt is burning, and then at a sheet of white paper. Does the after-image move with the eye?

Obtain an after-image by suddenly turning off the gas in a dark room, or by gazing at a window for half a second and then closing the eyes.

8. Negative After-images.-Having rested the eyes, gaze for about half a minute at a small white square on a black background. Suddenly place a sheet of white paper over the black. How does the present after-image differ from those obtained previously.

Look at a black square on a white ground; then suddenly let the eyes rest upon a gray screen. Observe the after-image, and note the apparent increase in its size when the gray screen is moved farther away from the eye.

Gaze at a bright red square upon a black ground; then look at a white surface. Does the bluish-green after-image show periodic varia-

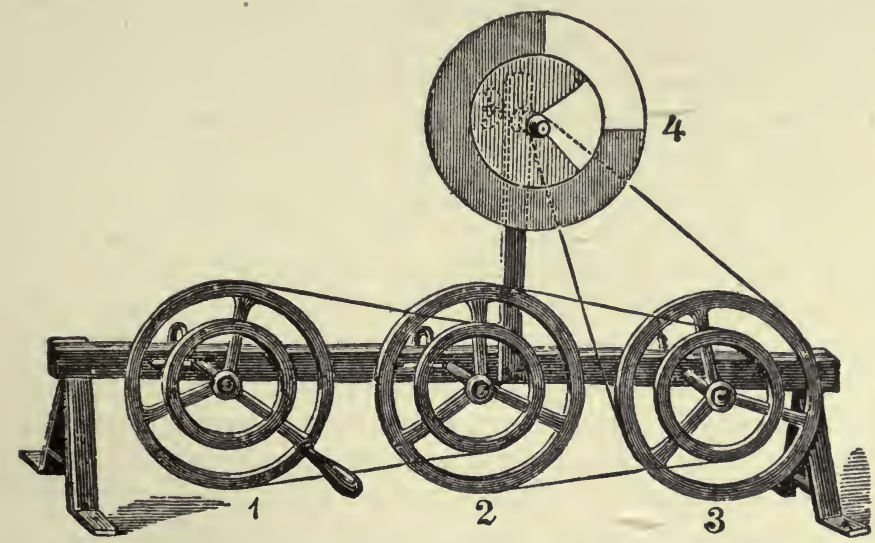

Fig. 115.-Rothe's Rotatory Apparatus for Color Disks. It is so Arranged as to Give Various Rates of Rotation by Combining the Motions of 1, 2, and 3.

tions? Repeat the experiment with a green square. Note that the color of the after-image is complementary to that of the square.

Place the red and green squares side by side on the black surface. Look at them steadily for about twenty seconds, and then slip a sheet of white paper over the whole.

9. Fusion of Gray and White.-By means of Maxwell's color mixer rotate the black and white disk supplied for this experiment until you have obtained complete fusion of the black and white in the outer zones of the disk.

Increase the frequency of the revolutions of the disk until even the central zones appear of a uniform gray color. Darken the room somewhat, and determine how many revolutions are necessary to produce a gray of the same quality as before.

10. Fusion of Colors.- Rotate two large disks, colored respectively red and green, and adjust the colored areas so as to obtain a dark yel- 
low. Match this color by inserting two sinall disks, black and yellow respectively. Obtain a blue color by means of two large disks colored green and yellow. Match the blue color by means of two small disks, respectively blue and black.

Use three disks colored red, green, and violet. The wave-lengths of these colors should correspond as closely as possible to those of the corresponding colors of the solar spectrum. Arrange the colored areas in the proportion of red, 118 degrees; green, 146 degrees; and violet, 96 degrees. Rotate the disks until you have obtained a gray color. Match this gray by means of two small disks, colored black and white.

11. Complementary Colors.- Select from the series of colored disks provided for this experiment one of a blue color. Determine what color, when mixed with this blue in definite proportion, will turn it gray or nearly white. The color-mixer should make from 40 to 50 revolutions in a minute. If the gray remain slightly tinged with color, add a small section of a third color disk, neutralizing with green if the tinge inclines toward red, and with red if the tinge exhibits a greenish hue. Determine in the same manner the colors complementary to red, yellow, orange, and green.

12. Intensity of Light and Quality of Color (Purkinje's Phenomenon).-Take two small squares of red and blue paper respectively, which in a moderately bright light possess nearly the same intensity of color. Observe them again in a partially darkened room. Which color appears to be the brighter?

13. Color-blindness.-Place Holmgren's worsteds upon a well lighted sheet of white paper. Employ as a test color light green, $i$. e., a mixture of white and pure green, and ask the subject to select from a lot of skeins all those which appear to him to be of this light green color. If he choose one or more "confusion-colors"-in this case pink or yellowhe should be given a skein of a pale rose color, and be asked to pick out all the skeins which he thinks would match it. If the subject be redblind, he will select blue and violet; if green-blind, gray and green.

Ask the subject to match a bright red skein. If red-blind, he will choose green and brown; if green-blind, red and light brown.

14. Contrast.-Of two equally large strips of gray paper, place one upon a sheet of white paper, and the other upon a sheet of black. Which strip appears to be the brighter?

Place these sheets close together, each sheet bearing its gray slip. Gaze at both slips for twenty seconds, and then shut the eyes. Which after-image seems the brighter?

Place a cross of gray paper in the middle of a large sheet of green paper and cover both cross and background with tissue paper. Note the color now imparted by the cross. Place the gray cross upon a red background instead of a green. Cover the whole with tissue paper as before and note the color of the cross.

Place a strip of gray paper across the junction of a red and a green 
sheet of paper and cover the whole with tissue paper. Note the apparent color of the strip.

15. Irradiation.-Place a circular piece of black paper $1 \mathrm{~cm}$. in diameter upon a white circle of paper $2 \mathrm{~cm}$. in diameter, and a white circle $1 \mathrm{~cm}$. in diameter upon a black circle $2 \mathrm{~cm}$. in diameter. Hold the circles at some distance from the eyes and note their apparent size.

Divide a square into four small squares of equal size, and blacken the left upper and the right lower of these four fields. Observe that the white squares appear to be larger than the black.

16. Single and Double Images.-Adjust your vision so as to see clearly with both eyes a vertical rod held $60 \mathrm{~cm}$. in front of them. Hold upright the index-finger of one hand in the binocular line about $35 \mathrm{~cm}$. from the eyes, and observe the double image of the finger. Close the right eye and note whether the right or the left image disappears. Accommodate for the finger, and observe the resulting double image of the rod. Close one eye and note which image disappears. Explain.

Accommodate for an object placed about $2 \mathrm{~m}$. in front of the eyes. Hold a pencil upright in the binocular line about $30 \mathrm{~cm}$. from the eyes. Move the pencil slightly toward the right eye. Observe that one image disappears when the images lie asymmetrically to one side of the line of vision only, the image in the right eye being perceptible in the present case. Close the right eye and in this way render the second image perceptible.

17. Binocular Fusion of Dissimilar Images.-Fasten a red and a green postage stamp upon a piece of cardboard at a distance equaling the interocular distance. Observe them in the stereoscope and note. that their images are fused into a single one.

18. Relation of Binocular Vision to Judgments of Direction.-Draw a sheet of paper, with a pinhole in it, horizontally past the eyes, starting well to the right of the eye. To what plane of the head are the two successive images referred?

19. Relation of Binocular Vision to Judgments of Solidity.-Examine a series of pictures in the stereoscope. Draw a diagram to show the manner in which judgments of solidity are formed.

20. Relation of Binocular Vision to Judgment of Distance.-Close one eye and hold the index-finger of the left hand vertically in front of the other eye. Try to strike the left index-finger with the indexfinger of the right hand.

Close one eye and try to dip the index-finger of one hand into the mouth of a bottle held about $25 \mathrm{~cm}$. in front of the other eye.

With the left hand hold a pencil vertically about $25 \mathrm{~cm}$. in front of the eyes. Gaze at the pencil for a few seconds, close the left eye; then cover the lower part of the pencil with your right index-finger held vertically between the object and the eyes. Try to strike the pencil with the finger.

21. Influence of Convergence of the Visual Axes.-Gaze at an 
object through two blackened tubes of paper. Cause the tubes to converge slowly and observe the apparent increase in the size of the object.

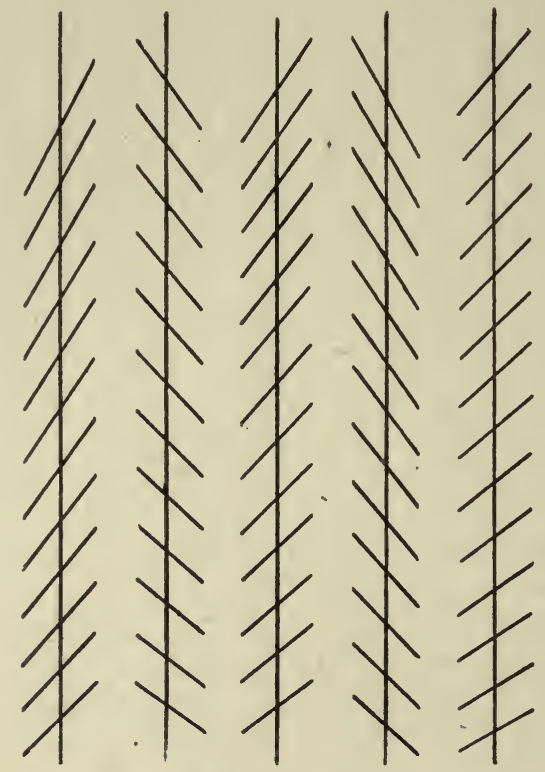

Fig. 116.-ZollNer's LiNes.

22. Optical Illusions.-Examine a series of figures, such as the following, and give reasons for the illusion:

$A$

$$
B
$$
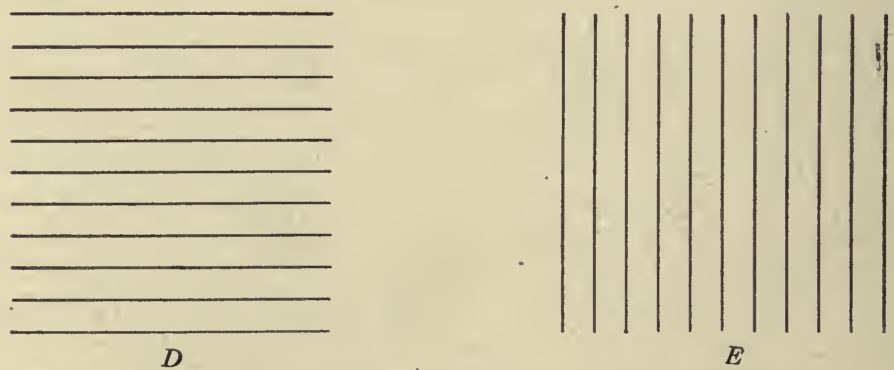

Fig. 117.-To Illustrate the Illusion of Subdivided Space.

(a) Make two lines parallel and draw short oblique lines through them. Note that the lines now slope in accordance with the direction of the oblique lines (Fig. 116).

(b) Make three black dots $(A, B$, and $C)$ at equal distances from 
one another. Between $A$ and $B$ make several dots of equal size. $A$ and $B$ will then appear farther apart than $B$ and $C$ (Fig. 117).

(c) Draw two horizontal lines of equal length and parallel to one another. Upon the ends of one draw oblique lines outward and upon

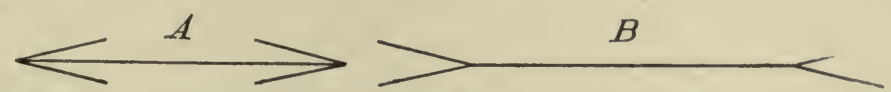

Fig. 118.-Muller-Lyer Figures to Show Illusion in Space Perception. The Lines $A$ and $B$ are of the Same Length.

the ends of the other similar lines turned inward. The latter will then appear to be shorter than the former.

(d) Draw two lines of equal length parallel to one another. Next to this figure draw another of the same dimensions with several inter-

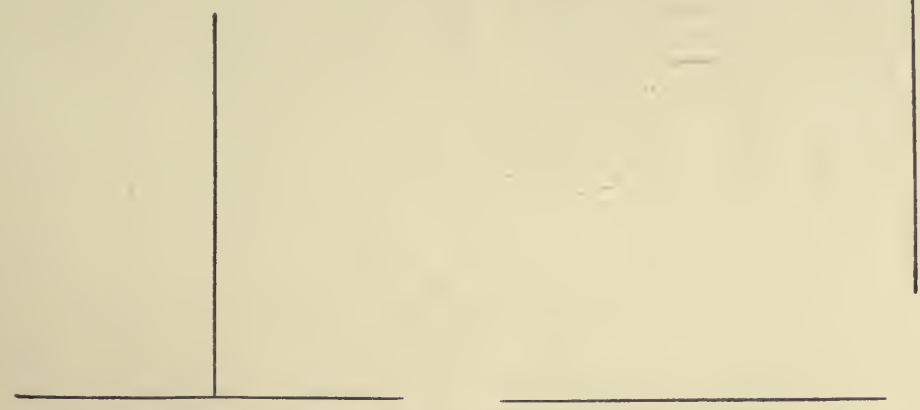

Fig. 119.-To Illustrate the Overestimation of Vertical Lines.

coursing lines. Note that the second figure appears to be larger than the first (Fig. 117).

(e) Place a vertical line beside a horizontal line of the same length. The former appears to be the longer (Fig. 119). 





\section{LESSON XLIII}

\section{DIGESTION}

\section{DEGIUTITION}

1. Isolation of the Esophagus.-Anesthetize a mammal and maintain the anesthesia throughout the following experiments: Perform tracheotomy low down. Separate the trachea from the esophagus, and excise the piece of trachea situated between the cricoid cartilage and the metal cannula. Palpate the esophagus, noting its texture and movability.

Expose both vagi nerves and place them in loose ligatures. In addition, expose their superior laryngeal branches and place them in loose ligatures.

Make a median incision through the linea alba. Follow the anterior wall of the stomach until the esophageal gastric junction has been reached. Insert a straight cannula in the orifice of the esophagus, and connect it with a manometer containing water. Cover the abdominal wound with a cloth moistened with warm saline solution.

2. Wave of Deglutition.-Open the mouth of the animal and touch the fauces with a moistened plug of cotton. Observe the swallowing movements evoked thereby. Note that they begin in the mouth and travel downward in the form of a peristaltic wave, involving the different segments of this passage consecutively.

Observe the displacement of the column of water, and determine approximately the interval of time between the beginning of this act and the moment when the wave has reached the level of the larynx, and again when it has arrived at the cardiac end of the stomach. In which segment is its progress slowest?

Evoke these movements by stimulating the central end of the superior laryngeal nerve.

3. Division of Esophagus.-Cut transversely across the cervical portion of the esophagus. Stimulate the central end of the superior laryngeal nerve, evoking peristalsis. Does the wave proceed as before, or is it blocked at the line of the section? Explain.

Raise the orifice of the lower segment slightly. Insert an elliptic piece of smooth wood secured by means of a thread. Does the introduction of this body lead to a wave of deglutition in this segment? If not, excite a wave in the normal way, either by stimulating the superior laryngeal nerve or by stimulating the mucous membrane of the mouth. Is the wood now propelled onward? Explain.

4. Influence of the Vagi Nerves.-Divide the right vagus nerve. Excite deglutition by stimulation of the superior laryngeal nerve. Study the character of the wave. Divide the left vagus nerve and repeat this 
stimulation. What differences do you observe? What part do the vagi nerves play in deglutition? Kill the animal by an overdose of ether. Open the thorax and trace the esophagus through the chest. Measure its total length, and determine the velocity of the wave of deglutition.

5. Deglutition in the Human Subject.-Let the subject take a swallow of water while you auscultate first over the region of the larynx and again over the cardiac orifice of the esophagus. Describe the character of the noises heard. Determine the approximate interval between them. Chew a piece of bread and swallow in the usual way. Determine the interval between the aforesaid noises. The time of deglutition is much longer in the latter instance.

6. Percussion of the Human Stomach.-Let the subject take sodium bicarbonate in conjunction with tartaric acid and assume the horizontal position. Percuss the region of the stomach, outlining this organ with colored chalk. Determine the position of the pyloric orifice. What relation does the lower boundary of the stomach bear to the umbilicus? Where would this boundary be found in a dilated organ? Explain the fact that gas is not erupted until the subject assumes the recumbent position. 




\section{DIGESTION (Continued)}

\section{SECRETION OF SALIVA}

1. Isolation of the Secretory Nerves.-Anesthetize a mammal and maintain the anesthesia throughout the following experiments until the animal has been killed. Perform tracheotomy. Expose the left common carotid artery and insert in its central end a straight cannula. On the opposite side make a longitudinal cut through the skin a little to one side of the median line, and beginning at a point opposite the canine teeth. Prolong it backward to the angle of the jaw. Cut through the platysma myoides. The next layer of muscle-fibers is arranged transversely. Carefully split this layer (musc. mylohyoideus) with forceps, reflecting its halves to the right and left. Let the assistant place his index-finger upon the floor of the mouth and pull it away from the side of the lower jaw. Identify the lingual nerve which passes transversely

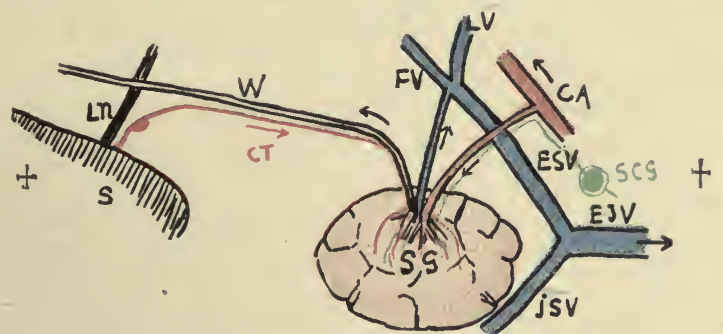

Fig. 120.-Schema Illugtrating the Nerve Supply of the Submaxillary Gland.

$S G$, Submaxillary gland; supplied by a small artery from the carotid system $(C A)$. It is drained by a small vein which generally enters the facial $(F V)$ at its point of confluence with the lingual vein $(L V)$. The external $(E S V)$ and internal $(J S V)$ maxillary veins invest the gland and unite to form the external jugular vein $(E J V)$. The sympathetic nerve supply is derived from the sup. cerv. ganglion $(S C G)$. The chorda tympani $(C T)$ attaches itself to the lingual nerve $L N$ and then to Wharton's duct $(W) ; S$, lower jaw.

across the floor of the mouth at about the junction of the middle and posterior thirds of the jaw. Follow the latter in a central direction until you reach the chorda tympani nerve and the duct of the submaxillary gland (Wharton's). Isolate the chorda tympani nerve at the point where it leaves the lingual nerve, and place it in shielded electrodes. Ligate the duct of the submaxillary gland and stimulate the chorda tympani for a few seconds, so as to distend the duct with saliva. Insert a cannula. Expose the vagosympathetic nerve of the same side, cut it, and arrange for stimulating its central end (toward the head) with a weak tetanizing current. 
2. Function of the Chorda Tympani Nerve.-Stimulate the chorda tympani for fifteen seconds and count the number of drops of saliva which escape during this time. Observe the degree of viscosity of the saliva. Pour it from one beaker into another, noting its viscidity. Repeat this test.

3. Function of the Sympathetic Nerve.-Stimulate the central end of the sympathetic nerve and count the number of drops of saliva. Observe its viscosity and viscidity. Contrast it with the chorda saliva.

4. Secretory Pressure.-Record on the kymograph the bloodpressure prevailing in the carotid artery of this animal. Disconnect the carotid artery from the manometer and connect the latter with the duct of the submaxillary gland. Stimulate the chorda tympani for a considerable time, with occasional intervals of rest, and record the pressure under which secretion still takes place. Allow the drum to revolve very slowly. The pressure in the salivary duct may be raised a considerable distance above the general blood-pressure, proving thereby that filtration is not the only factor concerned in the formation of this secretion. Enumerate the other factors.

5. The Vasomotor Changes in the Submaxillary Gland Produced by Stimulation of the Chorda Tympani and Sympathetic Nerve.-Reflect the skin and expose the large veins in the vicinity of the submaxillary gland. Ligate the internal maxillary and facial veins opposite the anterior end of the gland, and ligate the sublingual vein about $2 \mathrm{~cm}$. distally to its junction with the facial vein. Carefully destroy by torsion the larger cutaneous veins which empty their contents into the aforesaid veins centrally to the ligatures. The vein which returns the blood from the submaxillary gland pursues a very irregular course. In most cases, however, it joins one or the other of the above-mentioned veins at a point nearer to the heart than where the ligatures have been applied. If this relationship prevails in the mammal used for this experiment, only the blood returned from the submaxillary gland will be able to enter the external jugular vein.

Insert a cannula in the distal end of the external jugular vein near the point where the internal and external maxillary veins unite. Connect the cannula with a bottle having an inlet and an outlet tube, and containing a solution of magnesium sulphate. When the blood is allowed to flow into the bottle it will displace an equal quantity of this solution, which can be measured in a graduated cylinder.

Measure the blood flow for a short time, then stimulate the chorda tympani nerve until you have obtained a decided increase in the blood flow. Allow this flow to become normal again. Stimulate the sympathetic nerve until the blood flow has been considerably lessened. These results are referable to the fact that the chorda possesses vasodilator qualities, whereas the sympathetic is a vasoconstrictor nerve.

6. Secretion by the Bloodless Gland.-Render the submaxillary gland bloodless by ligating the blood-vessels supplying the head, or by decapitating the animal. Stimulate the chorda tympani, and measure 
the quantity of saliva secreted. How long after the cessation of the blood flow may saliva be obtained? What conclusion seems justified regarding the secretory activity of the cells of this gland?

7. Dissection of the Salivary Glands.- Isolate the sublingual and parotid glands and their excretory ducts.

Examine preparations of resting and active tissue from the parotid or submaxillary gland under the microscope. 





\section{LESSON XLV \\ DIGESTION (Continued)}

\section{SECRETION OF PANCREATIC JUICE. ACTION OF SECRETIN. GASTRO- ENTEROSTOMY}

1. Preparation of Secretin.-Anesthetize a mammal and maintain the anesthesia throughout the following experiments: Perform tracheotomy. Open the abdomen by an incision through the linea alba. Identify the pyloric sphincter. Beginning at this point isolate a segment of intestine about $1 \mathrm{~m}$. in length. Remove it; slit its wall, and wash it thoroughly in running water. Kill the animal by an overdose of ether. Scrape off the mucous lining of the segment of intestine just removed with a piece of glass, and immerse this material in saline solution and sand. Add to it three or four times its volume of 0.4 per cent. $\mathrm{HCl}$ and place it in the ice-chest. Shortly before being

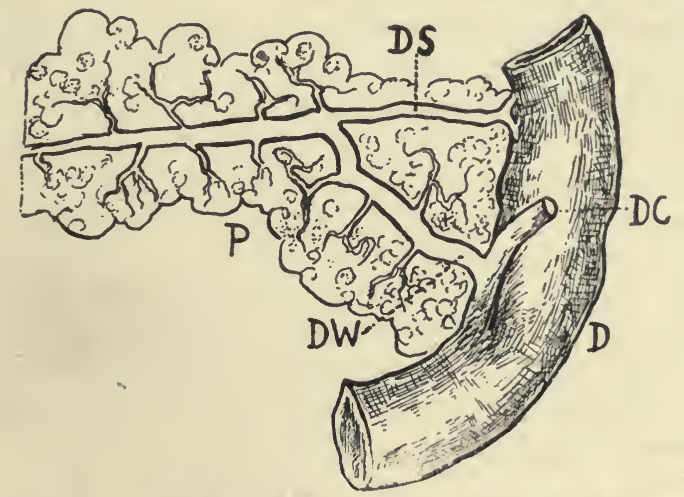

Fig. 121.-Diagram to Show the Position of the Ducts of the Pancreas. $D$, Duodenum; $P$, pancreas; $D C$, ductus choledochus; $D W$, ductus Wirsungianus; $D S$, ductus Santorini.

used add $\mathrm{KOH}$ to this decoction of the duodenal mucosa until changed to an alkaline reaction. Strain through muslin and filter. As much as 50 c.c. of this preparation should be used at a time. Since the acidified decoction may be kept for some time, it is advisable for economic reasons to prepare it from some of the animals used during one of the exercises immediately preceding. If an animal has been used especially for obtaining secretin, it should be kept until the end of this exercise for purposes of dissection.

2. Collection of Pancreatic Juice.-Anesthetize a mammal and maintain the anesthesia throughout the following experiments: Perform tracheotomy. Open the abdominal cavity by an incision in the linea 
alba. Identify the pyloric sphincter and duodenum. Note the position, shape, color, etc., of the pancreas. Inspect the body of this organ. Note how closely it invests the duodenum. Identify the duct of Wirsung. Open the duodenum in this region and locate the papilla through which the pancreatic and common bile-ducts discharge their contents. Insert a straight glass cannula in this orifice, and place a clip upon the common duct farther distally.

Adjust the mouth of the cannula so that the drops of pancreatic secretion may fall upon the spoon of a receiving tambour. By means of narrow rubber tubing connect this tambour with a recording drum registering the number of drops upon the paper of a slowly revolving kymograph. Add a time-curve.

3. Action of Secretin.-Expose the right external jugular vein and insert a cannula in its central end (see Lesson X). Slowly inject 50 c.c. of the activated preparation of duodenal mucosa. Register the drops of pancreatic juice in the manner just described. Compare the results. Disconnect the apparatus.

4. Gastro-enterostomy.-Procure curved needles, catgut, and operating instruments. Select a segment from the upper portion of the jejunum. Bring its flat surface against the region of the greater curvature of the stomach, avoiding the blood-vessels of this region. Approximate these surfaces, being careful to give a normal curvature to the duodenum. Make an incision about $6 \mathrm{~cm}$. in length in the anterior wall of the stomach at a safe distance from the blood-vessels passing along the greater curvature. Make a similar incision in the convex side of the loop of the jejunum. Suture the two orifices in apposition, first by an external serous suture carried along one side of the orifice and then by an internal row of sutures embracin the mucosa. Repeat on the other side in the reverse direction. What is the purpose of this operation? Discuss physiologic points for and against this operation. Under what circumstances may it produce beneficial results? Kill the animal by an overdose of ether. 




\section{LESSON XLVI}

\section{DIGESTION (Concluded)}

\section{LACTEALS AND THORACIC DUCT. PERISTALSIS. SECRETION OF BILE}

1. Lacteals.-Anesthetize a cat which has been fed with fatty food several hours beforehand, and maintain the anesthesia throughout the following experiments: Perform tracheotomy. Open the abdomen by a longitudinal cut in the median line, and thus expose the viscera. Throughout the subsequent experiments keep the viscera warm by applying cloths moistened with warm physiologic salt solution. Examine the walls of the intestine and the mesentery. Identify the lacteals filled with chyle, and note their beaded appearance. These constrictions indicate the positions of the lymphatic valves. Trace the lacteals to the receptaculum chyli, the enlarged abdominal end of the thoracic duct, which lies opposite the kidneys.

2. Peristalsis.-In the cat, spontaneous peristaltic movements of the small intestine are seldom in evidence. Give reasons for this motor quiescence. Stimulate the intestine mechanically and electrically and watch the resulting contractions.

Is the stomach contracting? Stimulate its surface at the fundus and at the pylorus. Compare the results.

Expose the right vagus nerve; cut it, and stimulate its distal stump. Observe the movements of the stomach and intestine. What is their character?

3. Chyle.-Slit open a distal lacteal. With a pipet transfer a few drops of chyle to a watch-glass and observe the coagulation. Place a drop on a glass slide and examine it microscopically, identifying white corpuscles and fat globules. Close the opening in the lacteal by means of an artery clamp.

Expose the region of the hepatic artery and trace the numerous lymphatics to the hilum of the liver. What is their appearance? What is the character of their contents?

4. Secretion of Bile.-Ligate the common bile-duct just above its entrance into the duodenum, and insert a cannula in its hepatic end. Empty the gall-bladder by gently squeezing it between your fingers and occlude the cystic duct by means of an artery clamp. Connect the cannula with a graduated cylinder. Read off the quantity of bile so far collected. Note the effect upon its level of the respiratory movements. Ascertain by reading the amount of bile that is secreted in a given period of time. After fifteen minutes place the graduated cylinder in a vertical position, and determine the pressure under which the bile is being secreted. What is the relationship between the secretory 
pressure and the blood-pressure in the portal vein? Obviously, the bilepressure is determined in this case in $\mathrm{mm} . \mathrm{H}_{2} \mathrm{O}$. Divide by 13.5 in order to obtain the corresponding value in $\mathrm{mm}$. Hg. Since the portal pressure in cats amounts to about $7 \mathrm{~mm}$. $\mathrm{Hg}$ while the secretory pressure frequently rises to $15 \mathrm{~mm}$. $\mathrm{Hg}$, it may be concluded that the hepatic cells are capable of secreting even against a pressure higher than that under which they obtain their material.

5. Experimental Jaundice.-Inject into the bile-duct a saturated solution of indigo-carmin, noting by a watch the time when the injection has been begun. Clamp the common duct. Watch for the blue color to appear in the skin, the mucous membrane of the mouth, the conjunctiva, and the chyle in the lacteals. Squeeze the urinary bladder at intervals and expel a small quantity of urine. How soon after the beginning of the injection does the urine assume a blue color?

6. Thoracic Duct.-Arrange for artificial respiration. Open the chest and expose the thoracic duct on the left side of the spinal column. It is now rendered clearly visible by the blue chyle.

7. The Stomach Contents.-Palpate the stomach. Has the food been fully reduced mechanically? Evacuate its contents. Wash the gastric mucosa and note its soft velvety texture. Examine preparations of gastric glands under the low and high powers of a microscope. Identify the chief and parietal cells. 




\section{LESSON XLVII}

\section{ABSORPTION}

OSMOSIS. INTESTINAL PERISTALSIS, SECRETION OF INTESTINAL JUICE. ABSORPTION FROM THE SMALL INTESTINE

1. Osmosis.-Prepare an osmometer by tying a piece of fish condom or other semipermeable membrane over the orifice of a thistle tube. Fill the chamber of this tube with a concentrated solution of magnesium sulphate. Fill a beaker with distilled water and suspend the filled thistle tube in the water so that the levels of the fluids agree. Attach a narrow glass tube, about $1 \mathrm{~m}$. in length, to the end of the thistle tube, and allow the apparatus to stand for twenty-four hours.

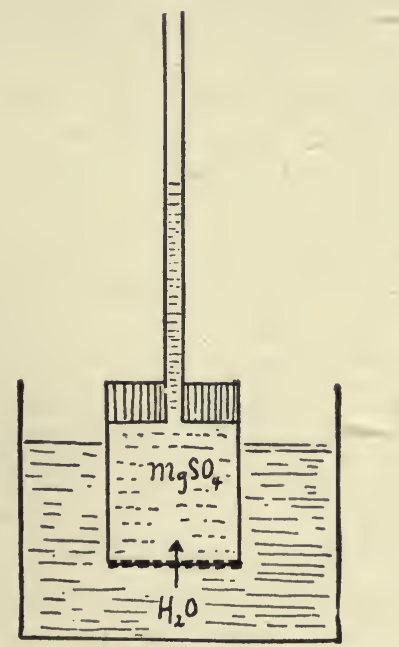

Fig. 122.-A Simple Osmometer.

The receptacle contains water, and the cell a solution of magnesium sulphate. As the molecules of water are drawn through the semipermeable membrane the level of the $\mathrm{MgSO}_{4}$ solution rises.

Already in the course of an hour or two it will be observed that the level of the solution of magnesium sulphate has risen a considerable distance. The solution of magnesium sulphate possesses a greater osmotic pressure and draws molecules of water through the membrane.

2. The Osmotic Power of the Intestine.-Anesthetize a cat and maintain the anesthesia until the animal has been killed by an overdose of ether. Perform tracheotomy. Open the abdominal cavity by a small longitudinal incision in the linea alba. Pull a loop of small in- 
testine about $20 \mathrm{~cm}$. in length through this opening. The jejunum is usually empty. If not, gently squeeze its contents along into the lower bowel. Tie a ligature tightly about the middle of the exposed segment, and a second and third about $8 \mathrm{~cm}$. distally and centrally to this one. In this way two segments of intestine have been isolated. Into one of these inject 5 c.c. of a saturated solution of magnesium sulphate, and into the other 30 c.c. of a 0.7 per cent. solution of sodium chlorid. Note that the former segment feels perfectly empty, whereas the latter is highly distended. Replace the entire loop in the abdominal cavity, marking its upper end with a colored piece of cord. Close the opening in the abdominal wall with a clamp. Cover the animal with a warm cloth and allow it to rest under anesthesia for thirty minutes. At the end of this period expose the aforesaid segments and determine their degree of distention. It will now be found that the loop containing the $\mathrm{Mg} \mathrm{SO}_{4}$ is highly distended, whereas the loop with the $\mathrm{NaCl}$ is practically empty. Obviously, the $\mathrm{Mg} \mathrm{SO}_{4}$, possessing a greater osmotic pressure.than the body fluids, has abstracted water from the latter. Contrariwise, the 0.7 per cent. solution of $\mathrm{NaCl}$ has been removed from the loop. Using these facts as a basis, explain the action of the saline cathartics.

3. Chemical Stimulation of the Secretion of Intestinal Juice.Insert a cannula in the external jugular vein, and empty a loop of duodenum about $30 \mathrm{~cm}$. in length. Inject intravenously from 1 to 3 c.c. of $\mathrm{M} / 8$ solution of barium chlorid or sodium citrate. Observe the peristalsis which results almost immediately and measure the quantity of intestinal juice obtainable from this segment. Kill the animal by an excessive amount of ether.

4. Gastric Fistula.-Procure curved needles, a needle-holder, and clamps. Bring the wall of the pylorus in the wound and anchor it by means of a continuous suture. Decrease the length of the wound by sutures. Incise the gastric wall and suture it to the edges of the wound. Insert a gastric cannula and secure it by additional sutures.

5. Peristalsis.-Anesthetize a rabbit and maintain the anesthesia during the following experiments and until the animal has been killed by an overdose of ether. Perform tracheotomy. Open the abdominal cavity by a median incision through the linea alba. Observe the peristalsis. The intestine of the rabbit invariably shows most intense peristaltic movements when exposed to the cooler air or stimulated mechanically. Note the pendular movements and also the regular peristalsis.

Compare the length and general appearance of the intestines of the rabbit with those of the intestines of the cat and dog, noting especially the predominance of the large intestine in the former animal. Discuss the functional significance of this difference.

6. The Influence of Salts on Peristalsis.-Apply a few drops of $\mathrm{M} / 320$ solution of barium chlorid or sodium citrate to the peritoneal surface of a loop of intestine. Observe the marked peristalsis which 
follows. Inhibit this movement by applying a solution of calcium chlorid or magnesium chlorid.

7. Secretion of Intestinal Juice into Excised Loops.--Empty a loop of small intestine about $10 \mathrm{~cm}$. in length by gentle pressure exerted with your fingers. Place two pairs of ligatures around the intestine so as to isolate this segment. Ligate the corresponding blood-vessels. Remove this segment from the body by cutting between each pair of ligatures. Kill the animal by an overdose of ether. Suspend the excised loop at $38^{\circ} \mathrm{C}$. in a measured quantity of $\mathrm{M} / 8$ sodium chlorid to which from one-seventieth to one-fiftieth of its volume of $\mathrm{M} / 8$ barium chlorid has been added. Keep the ends of the intestinal segment above the surface of the solution. Observe the peristalsis. After about twenty minutes measure the quantity of fluid which has been seereted into the lumen of the segment. Since the quantity of the fluid surrounding this loop has not been lessened, the secretion must have been formed from the material stored in the cells at the time of their removal from the body.

8. Influence of Adrenal Extract on Intestine.-Remove a piece of duodenum, about $10 \mathrm{~cm}$. in length, and place it in cold Ringer's solution. After a time cut off a piece $2 \mathrm{~cm}$. in length and suspend it in a beaker filled with Ringer's solution. Connect its lower end with a weight placed upon the bottom of the receptacle and its upper end with a writing lever properly counterpoised.

Place a small flame underneath the beaker and slowly raise the temperature of the fluid to $30^{\circ} \mathrm{C}$. Start the recording drum and register the movements of this strip.

Add 0.5 c.c. of adrenalin to the Ringer fluid (300 c.c.). Note the effect upon the length of the strip (tonus) as well as upon its contractions.

9. Rapidity of Absorption and Elimination.-Into each of ten testtubes pour 5 c.c. of thin starch paste and 2 c.c. of concentrated nitric acid. Swallow a capsule containing 10 grains of potassium iodid and rinse your mouth thoroughly. Increase the secretion of saliva by chewing a piece of paraffin and collect at intervals of two minutes a quantity of saliva in the corresponding test-tubes. Rinse the mouth after each collection. How soon after ingestion can this agent be recognized in the saliva?

10. Histologic Examination of Preparations of Intestinal Mucosa.Study the structure of a villus, mucous cells, and glands of Lieberkühn. 


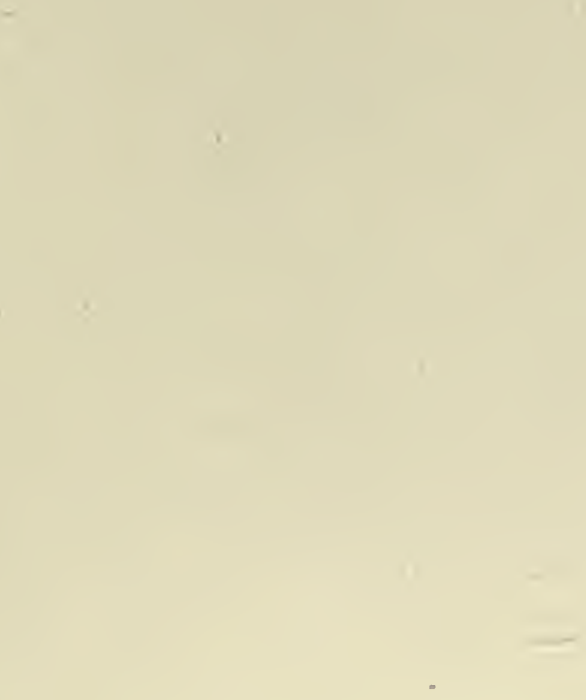

- 



\section{LESSON XLVIII}

\section{EXCRETION}

\section{SECRETION OF URINE}

1. The Drop Method of Registering the Flow of Urine.-Anesthetize a mammal and continue the anesthesia throughout the following experiments: Perform tracheotomy. Expose the left common carotid artery and insert in it a straight glass cannula. Fill it with a solution of sodium carbonate, and connect it with the mercury manometer. Isolate the vagus nerve on the same side and place it in a loose silk ligature. On the opposite side expose the external jugular vein. Clamp it centrally; ligate it about $2 \mathrm{~cm}$. distally to the clamp, and insert a straight glass cannula toward the heart. Fill this cannula with normal saline solution.

Open the abdominal cavity in the linea alba below the umbilicus. Identify the bladder and the two ureters leading away from its posterior surface. Insert a straight cannula in each (toward the kidney) and connect them by means of short pieces of rubber tubing with a Y-tube. Allow the end of the Y-tube to project beyond the edge of the board, at a distance of about $20 \mathrm{~cm}$. above the spoon-shaped lever of the receiving tambour. Connect the latter by means of a long piece of rubber tubing with a recording tambour placed against the paper of the kymograph. Adjust the recording needle of the mercury manometer in such a way that it registers in the same ordinate as the writing lever of the recording tambour. Allow a chronograph to register seconds below these levers.

2. Normal Secretion of Urine.-Allow the kymograph to revolve at a moderate speed, and register the drops of urine secreted in relation with the curve of the blood-pressure. Remember that the formation of urine is often greatly lessened during ether narcosis. If this condition prevails in the animal used for this experiment, stimulate the flow in the manner described in paragraph 3.

3. Action of Glucose.-Prepare a concentrated solution of glucose (25 c.c.). Filter it and draw 10 c.c. of the filtrate into a pipet. Connect the latter with the cannula inserted in the external jugular vein. Allow this quantity of glucose to enter the circulation, but slowly, so that the height of the blood-pressure is not altered. After a certain latent period drops of urine will be seen to enter the Y-tube at intervals. Observe the rapidity with which they are secreted. It is said that glucose stimulates the renal cells directly, giving rise to diuresis. Record the flow in relation with the blood-pressure.

4. Action of Sodium Chlorid.-When the diuresis produced by the glucose has nearly subsided, inject 100 c.c. of warmed saline solution. Register the flow of urine in the manner described above. Repeat the injection if the effect is not sufficiently decisive. 
The injection of so large a quantity of solvent is prone to produce a material rise in the blood-pressure, thereby increasing the filtration pressure. For this reason the diuretic effect of sodium chlorid is frequently referred to this cause, in combination with hydremia and osmotic changes, and not to a direct stimulating action upon the renal cells.

5. Effect of Changes in Blood-pressure.-When an active secretion has been produced, stimulate the vagus nerve, thereby evoking a material reduction in the general blood-pressure. While the secretion of urine is then greatly lessened, note that the flow does not return to normal until a considerable time after the cessation of the stimulation. This fact tends to show that while the blood-pressure is an important factor in the production of urine, the secreting cells are not dominated by pressure alone.

Dilute a $1: 1000$ solution of adrenalin sufficiently to cause a moderate rise in blood-pressure ( 2 c.c. of the solution to 20 c.c. of saline). Register the flow of urine. Inject a small quantity of the aforesaid

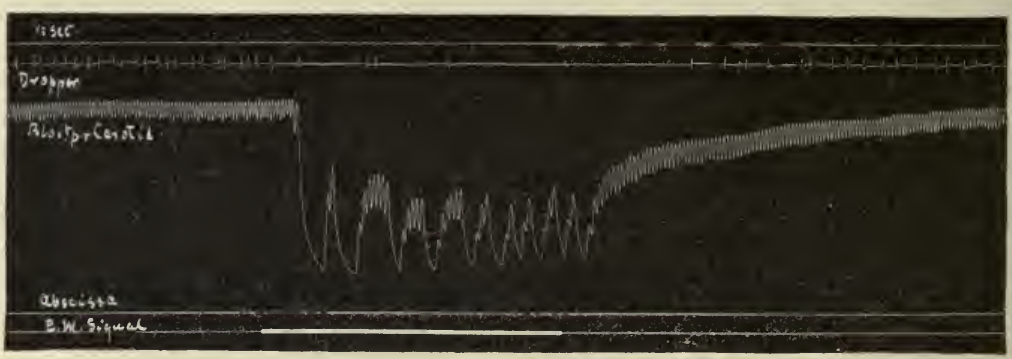

Fig. 123.-Effect of Stimulation of the Vagus Nerve Upon the Secretion of URINE.

solution of adrenalin in the external jugular vein. Note that the flow of urine is greatly lessened thereby in spite of the high blood-pressure. This discrepancy is easily explained, because the adrenalin constricts the blood-vessels of the kidney, and gives rise to a lessened vascularity of this organ and lessened secretory power of its cells.

6. Rapidity of Elimination.-Prepare a saturated solution of indigocarmin. Inject 5 c.c. of this solution in the external jugular vein, noting the moment of the injection. Again determine the time when this pigment appears in the urine. Kill the animal by an overdose of ether.

7. Dissection of the Region of the Kidney.-Identify the suprarenal bodies on each side. Carefully remove the left organ and expose the suprarenal plexus, greater and lesser splanchnic nerves, and fibers of the renal plexus.

Open the pelvis of the kidney and study the characteristics of the cut surface of this organ. Identify the papillæ, pyramids, medulla, cortex, and capsule. Remove the capsule. Is it closely adherent to the substance of the kidney? 




\section{LESSON XLIX \\ EXCRETION (Continued)}

\section{SECRETION OF SWEAT. BODY TEMPERATURE}

1. Sweat Nerves.-Anesthetize a cat and maintain the anesthesia until the animal has been killed by an overdose of ether. Expose the sciatic nerve, apply a ligature, and cut centrally to the ligature. Stimulate the distal end of the divided nerve with a quickly interrupted current of moderate intensity. Observe the beads of sweat collecting upon the pads of the feet. Take the rectal temperature of this animal. Apply a cloth moistened with warm water to the upper part of its body, thereby raising its body temperature. Sweating is also evoked by this means. Explain its purpose.

Expose the external jugular vein and inject 3 milligrams of atropin sulphate. Note that the stimulation of the sciatic nerve now remains ineffective. The atropin paralyzes the secretory nerve endings.

Inject 10 milligrams of pilocarpin. Sweat will again be secreted, because this agent excites the cells of the sweat-glands directly. Kill the animal by an overdose of ether.

2. Sweating in Man.-Procure a piece of paper sensitized with silver nitrate. Cleanse the palm of the hand and after a certain interval apply the paper to this surface. The orifices of the sweat-glands will be marked upon the paper as spots of silver chlorid. Apply to this area a small pad of cotton moistened with a 1 per cent. solution of atropin sulphate. Repeat the aforesaid test. Since atropin paralyzes the endings of the secretory nerves, this test will now remain negative.

3. Body Temperature.-Determine the body temperature of the subject by means of an ordinary thermometer, the bulb of which is placed beneath the tongue. Ask the subject to close the lips. Observe the rapidity with which the temperature becomes constant. How long a time must the thermometer be left in situ before the mercury remains stationary?

Determine the axillary temperature in the same manner. Compare.

Place the bulb of the thermometer in the palm of the closed hand. Read the temperature. Compare. Wrap the hand in a thick woolen cloth. Why is the temperature now higher? Place the same hand in water of $20^{\circ} \mathrm{C}$. for thirty seconds. Again determine its temperature. Explain the result.

Request the subject to make thirty flexions of the arms and legs. Quickly determine the body temperature. For how long a time does the rise persist? 





\section{LESSON L}

\section{EXCRETION (Concluded)}

\section{THE INNERVATION OF THE BLADDER. PILOMOTOR REACTIONS}

1. The Function of the Hypogastric Nerves.-Anesthetize a cat and maintain the anesthesia throughout the following experiments: Perform tracheotomy. Open the abdominal cavity by a median incision in the linea alba. Identify the urinary bladder, and raise its fundus sufficiently to expose the fatty tissue investing its cervical portion. By careful dissection isolate the nerve-fibers which ascend from here to the fundus. Place them in shielded electrodes, and arrange the electric apparatus for stimulation with a tetanizing current of medium strength.

Insert a small hook in the top of the fundus of the bladder and connect it by means of a thread with the end of a writing lever (suspension method). Counterpoise the lever so as to place the musculature of the bladder under a certain tension. Place cotton moistened with warm saline solution around the base of the bladder to protect the abdominal organs against evaporation and thermal influences. Allow the drum to revolve at a slow rate and stimulate the hypogastric fibers until a contraction of moderate height has been obtained.

Cut one nerve and stimulate the central end of this nerve. Note that the bladder is now made to contract reflexly through the hypogastric center and intact nerve on the opposite side.

2. Pilomotor Effects.-Unite the margins of the wound in the abdomen by a few sutures. Make a median incision through the skin covering the base of the tail and posterior extent of the vertebral column. Reflect the muscles. Clip away the spinal processes and adjoining laminæ of several vertebræ near the base of the tail. Apply a cotton tampon until the bleeding has stopped. Incise the dura mater and identify the chorda equinæ. Isolate several of its constituent nerve-fibers close to the base of the tail and place them in loose ligatures. Smooth the hairs of the tail and place the latter upon a sheet of white paper. Note its volume. Stimulate the nerve-fibers just isolated successively with a weak tetanizing current, until one is found which causes an erection of the hairs of the tail. Again smooth the tail and repeat this experiment. Explain the mechanism by which hairs are erected. Kill the animal by an overdose of ether. Repeat the stimulation of these pilomotor fibers after an interval of several minutes. Note that this mechanism remains effective for some time after death. 




\section{DEMONSTRATIONS TO BE GIVEN IN CONNECTION WITH THE PRECEDING LESSONS}

1. (a) The determination of the strength of the make and break induction shocks by means of the galvanometer. Projection method.

(b) The production of acid in contracting muscle. Acid fuchsin method.

2. Electrotonus. Pflüger's law of contraction.

3. The current of injury and current of action in muscle and nerve. Projection method.

4. The action of electric currents of high voltage.

5. (a) Extraction of the gases of the blood by means of the mercury pump. Barcroft-Haldane blood-gas apparatus.

(b) Viscosimeter (Burton-Opitz).

6. (a) Projection of the spectrum. Formation of the bands of oxyhemoglobin and reduced hemoglobin.

(b) The capillary circulation in the mesentery or bladder of the frog. Projection method.

7. (a) The action of the valves of the heart. Gad's method.

(b) The vitelline area in the developing chick.

8. (a) The construction and action of different types of galvanometers.

(b) Einthoven's string galvanometer and the action current of the heart.

9. Electrocardiography. Normal and abnormal records.

10. The registration of the sounds of the heart. Normal and abnormal records.

11. The effect of increases in intracranial pressure upon the circulation and respiration.

12. The blood-supply of the intestines, demonstrated by means of the recording stromuhr of Burton-Opitz. Stimulation of the splanchnic nerve.

13. The vasomotor function of the spinal cord. Division and stimulation of the cord.

14. Fluoroscopic examination of the heart and lungs in man.

15. Perfusion of the excised mammalian heart.

16. The application of the polygraph (Mackenzie).

17. Projection of the larynx. Stimulation of the superior and inferior laryngeal nerves.

18. Abolished and exaggerated reflexes in man in consequence of "high" and "low" lesions of the nervous system.

19. (a) The decerebrated pigeon.

(b) The decerebrated cat. 
20. (a) Lesions of the cerebellum in pigeons.

(b) Cerebellar defects in man. Nystagmus.

21. (a) Stimulation of the semicircular canals in the turtle.

(b) Lesions of the semicircular canals in pigeons.

22. Hemiplegia, aphasia, and hemianopsia in man.

23. The dissociation of the cutaneous sensations. Syringomyelia. Sensory anesthesia.

24. The accommodation of the eye of the frog, turtle, and fish.

25. (a) Large demonstration ophthalmoscope.

(b) Astigmatism determined by means of the ophthalmotonometer of Helmholtz.

26. Myopia and hypermetropia displayed by means of a large Kühne's artificial eye.

- 27. Fluoroscopic observation of the movements of the stomach and intestines in a mammal.

28. Removal of the thyroid and parathyroid bodies.

29. Removal of the suprarenal bodies.

30. (a) Dog with gastric fistula.

(b) Diffusion demonstrated with the help of the apparatus described by Abel. 


\section{WEIGHTS AND MEASURES*}

IN the metric system the liter is a unit of capacity equivalent to the volume occupied by the mass of 1 kilogram of pure water at its maximum density. It is equivalent in volume to 1.000027 cubic decimeter. Under this definition a milliliter ( 0.001 of a liter) is different from a cubic centimeter by a very minute fraction. However, as "cubic centimeter" is the term used throughout medical literature we shall use it in this book, though both the U.S. and British Pharmacopœias have adopted the term "milliliter" (mil.) in its place.

\section{A. METRIC}

Weight:

1 milligram (mg.)

10 milligrams $=1$ centigram (cg.)...........

10 centigrams $=1$ decigram (dg.) $\ldots \ldots \ldots \ldots \ldots$

10 decigrams $=1$ gram $(\mathrm{gm}.) . \ldots \ldots \ldots \ldots \ldots$

1000 grams $=1$ kilogram (kilo.) $\ldots \ldots \ldots \ldots \ldots \ldots$

Volume:

1 milliliter (mil.) .....................

1 cubic centimeter (c.c.) $\ldots \ldots \ldots \ldots \ldots \ldots \ldots$ (1 c.c. of water weighs $1 \mathrm{gm}$.)

1000 cubic centimeters $=1$ liter (L.).

\section{Length:}

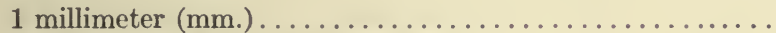

10 millimeters $=1$ centimeter $(\mathrm{cm}.) \ldots \ldots \ldots \ldots \ldots \ldots$

10 centimeters $=1$ decimeter $(\mathrm{dm}.) \ldots \ldots \ldots \ldots \ldots \ldots \ldots$

10 decimeters $=1$ meter $($ M. $) \ldots \ldots \ldots \ldots \ldots \ldots \ldots \ldots$
Approximate equivalent.

$\frac{1}{65}$ grain

$\frac{1}{6}$ grain

$1 \frac{1}{2}$ grains

15 grains

$2 \frac{1}{5}$ pounds

15 minims

15 minims

34 fluidounces

$\frac{1}{25}$ inch

inch

4 inches

40 inches

\section{B. APOTHECARIES}

Weight (Troy):

Approximate equivalent.

1 grain (gr.)

$0.065 \mathrm{gm}$.

10 grains

$0.7 \mathrm{gm}$.

20 grains $=1$ scruple $(\dot{\oplus}) \cdots \cdots \cdots \cdots \cdots \cdots \cdots \cdots \cdots \cdots \cdots$

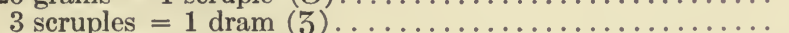

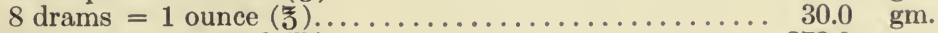

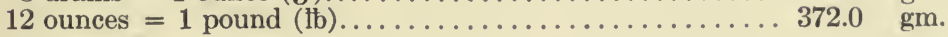

\section{Volume:}

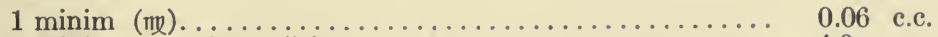

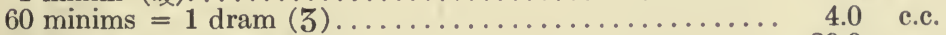

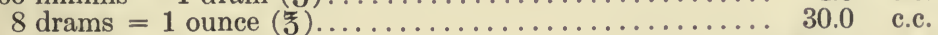

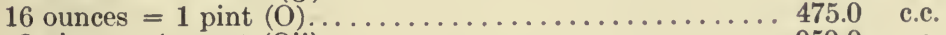

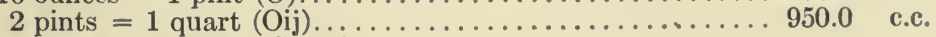

8 pints $=1$ gallon (Cong.).

( 1 gill $=4$ fluidounces.)

\section{Length:}

1 inch (in.)

* From Bastedo's "Materia Medica, Pharmacology, Therapeutics, and Prescription Writing." 
Noteworthy Terms:

Approximate

1 ounce avoirdupois. equivalent.

1 ounce troy 437.5

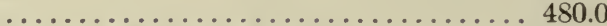

1 fluidounce of water (the standard of volume) . . . . 455.7

1 pound avoirdupois is . . . . . . . . . . . . . . . 7000.0

1 pound troy is. ................................

1 minim of water weighs $\frac{455.7}{480}$ grains $=0.95$ grain $=61.61 \mathrm{mg}$.

15 grains of water $=16$ minims; 1 grain of water measures 1.05 minims $=0.0648$ c.c.

An imperial pint is 20 ounces; a United States pint is 16 ounces.

EXACT EQUIVALENTS OF METRIC AND APOTHECARIES' WEIGHTS AND MEASURES ACCORDING TO THE U. S. PHARMACOPCEIA

\section{Volume:}

1 c.c

1 liter ( 1000 c.c.)

1 minim (nx).

1 fluidram (3).

1 fluidounce (§)

1 pint (O).

\section{Weight:}

1 milligram, 0.001 (mg.)

1 centigram, 0.01 (cg.)

1 decigram, 0.1 (dg.)

1 gram, 1.0 (gm.)

30 grams, 30.0

31 grams.

1 grain (gr.)

10 grains

15 grains.

1 scruple (円)

1 dram (3).

1 ounce troy (

1 ounce avoirdupois.
Approximate equivalent.

16.23 minims $33.8 \mathrm{oz}$.

0.061 c.c. 3.696 c.c. 29.57 c.c. 473.18 c.c.

$\begin{array}{cl}0.0154 & \text { grain } \\ 0.1543 & \text { grain } \\ 1.543 & \text { grains } \\ 15.4324 & \text { grains } \\ 462.9 & \text { grains } \\ 478.4 & \text { grains } \\ 0.065 & \text { gm. } \\ 0.648 & \text { gm. } \\ 0.972 & \text { gm. } \\ 1.296 & \text { gm. } \\ 3.89 & \text { gm. } \\ 31.1 & \text { gm. } \\ 28.35 & \text { gm. }\end{array}$
0.1543 grain 1.543 grains 15.4324 grains 462.9 grains 478.4 grains $\mathrm{gm}$

$0.648 \mathrm{gm}$

$0.972 \mathrm{gm}$.

$1.296 \mathrm{gm}$ 31.1 gm $28.35 \mathrm{gm}$. 


\section{INDEX}

AbDominal aorta, compression of, 107

Abduction of vocal cords, 143

Aberration, achromatic, 189 spheric, 190

Absorption, 217 and elimination, rapidity of, 219

Acceleration of heart, 88

Accommodation reflex, 184

Achromatic aberration, 189

Action current of frog's heart, 60 of muscle, 59

of nerve, 60

of cocain, 167

of glucose, 221

of secretin, 214

of sodium chlorid, 221

Acuity of dynamic sense, 180

of temperature sense, 170

Adaptation to touch sensations, 168

Adduction of vocal cords, 143

Adrenal extract, influence of, on intestine, 219

Adrenalin, effect of, on blood-pressure, 110

Afferent impulses, summation of, 157

After-images, negative, 201

of temperature, 169

positive, 200

Air, entrance of, into circulation, 115

All-or-none law, 82

Ameboid motion, 21

Ametropic artificial eye, ophthalmoscopic examination of, by direct method, 195

eye, 188,189

Amyl nitrite, effect of, on blood-pressure, 110

Anelectrotonus, 63

Anodic excitation of nerve, 64

Aorta, abdominal, compression of, 107

Aortic insufficiency, schema of, 102 stenosis, schema of, 102

Apex-beat of heart, 95

Apothecaries' weight, 229 exact equivalent, 230

Aristotle's experiment, 167

Arterial blood, 68

blood-pressure, direct method of determining, 103

pulse, character and velocity, 129

heart action and relation between, 129
Artificial eye, ametropic, ophthalmoscopic examination of, by direct method, 195

emmetropic, ophthalmoscopic examination of, by direct method, 194

opthalmoscopic examination of, by indirect method, 196

respiration, 140

Asphyetic blood, 68

Asphyxia, 135

Associated movements of eyes, 200

Astigmatism, 189

detection of, 194

measurement of, 194

Atmospheric pressure, decreased, effect on lungs, 148

Atropin, action on heart, 87

Auditory fatigue, 174

Auscultation, 139

Axes, visual, convergence of, influence of, 203

BABINSKI phenomenon, eliciting, 160

Bile, secretion of, 215

Binocular fusion of dissimilar images, 203 vision, relation of, to judgments of direction, 203

of distance, 203

of solidity, 203

Bladder, innervation of, 225

Blind spot, 199

contours of, 199

Blood, arterial, 68

asphyctic, 68

cells, red, human, counting of, 73

chemical tests for, 78

coagulation of, 69

corpuscles, red, enumeration, 70 simultaneous count, 73

relative amounts, 70

white, enumeration of, 71 simultaneous count, 73

crystals, 78

experiments on, preparation of animal for, 67

flow through lungs, effect of variations in intrathoracic pressure on, 147 velocity of, differences in, schema illustrating, 103 
Blood, medicolegal tests for, 77 microscopic examination, 73 plasma, relative amounts, 70 specific gravity, 75 spectroscopic examination of, 77 suspected, examination, 78 venous, 68

Bloodless gland, secretion by, 210

Blood-pressure, arterial, direct method of determining, 103

carotid, record of, 113

effect of adrenalin on, 110

of amyl nitrite on, 110

of changes in, 222

of division of both vagi nerves on, 114 of one vagus nerve on, 114

of exercise on, 128

of hemorrhage and saline injection on, 111

of posture on, 127

of stimulation of intact vagi nerves on, 113

estimation by indirect method, 125

influence of dyspnea on, 109

of hemorrhage on, 108

of posture on, 107

intracardiac, 115

venous, 128

Blood-supply, influence of, on muscle contraction, 52

Blood-vessels, retinal, 199

Body temperature, 223

Bones, manner of attachment of muscles to, 39

Capillary circulation, 99 in frog's lung, 147

Carbon dioxid, elimination of, in respiration, 151

influence of, in respiration, 140

Cardiac plethysmograph, 92

Cardiogram, 96

Cardiograph, 96

Cardiometer, 92

Carotid blood-pressure, record of, 113

Catelectrotonus, 63

Cathodic excitation of nerve, 64

Cell, Daniell, 25

dry, construction and action, 25

Cerebral localization, 163

Cerebrum, influence of, 162

removal of, effect, 161

stimulation of, 163

Cervical sympathetic nerve, vasomotor action, 117

Chemical and electric stimulation of temperature spots, 169

stimulation of muscle, 47

of secretion of intestinal juice, 218 tests for blood, 78

Chemicals, influence of, on muscle contraction, 48

Chloroform and ether, action of, on heart, 90
Chorda tympani nerve, function of, 210

Chyle, 215

Ciliary motion, 21

Circles of dispersion, 188

Circuit, primary, 29 secondary, 30

Circulation, capillary, 99

in frog's lung, 147

constant-flow, 99

entrance of air into, 115

in frog's lung, 147

intermittent flow, 99

schema, normal, 101

remittent flow, 99

schema, 100

Coagulation of blood, 69

Cocain, action of, 167

Coil, induction, 29

Cold and hot spots, mechanical stimulation of, 169

thermal stimulation of, 169

Cold-blooded animals, speed of nerve impulse in, 55

Collapse of lung, 136

Collection of pancreatic juice, 213

Color-blindness, 202

Colored objects, fields for, 191

Colors, complementary, 202

contrast, 202

fusion of, 201

Commutator, Pohl's, 56

Complementary colors, 202

Conductivity of nerve, 36

Constant current, 29

Contraction, muscle, compound, 43

effect of excessive stimulation on, 49 of veratrin on, 49

on volume, 38

fusion of, 42

height of, relation of amount of load to, 44

of strength of stimulus to, 43

human, 51

influence of blood-supply on, 52

of chemicals on, 48

of temperature changes on, 47

method of registering, 33

paradoxic, 60

single, 41

summation of, 42

Contrast colors, 202

Convergence of visual axes, influence of, 203

Corpuscles, blood. See Blood corpuscles. tactile, histologic examination of, 167

Crystals, blood, 78

hemin, 78

Currents, electric, types, 28

Cutaneous and muscular sensations, 167

Daniell cell, 25

Deglutition, 207

in human subject, 208

wave of, 207 
Depresion nerve, vasomotor action of, $11 \mathrm{~s}$

Diaphragm, action of, 135

Didg(on sphygmogr:uph, 12!)

Digestion, 207, 209, 21:3, 215)

Direct and indirect vision, 200)

method for ophthalmoscopic (xaminattion of anctropice artificial eye, 195

of ('mmetropic artificial ('y' (', 19)!

Direction, judgments of, relation of binocular vision to, $2(0) 3$

Diserimination of weight, Weber's law, 170

Dispersion, circles of, 188

Dissection of ear of dog-fish, 179 of eye, 18:3

of region of kidney, 222

of salivary glands, 211

Distanee, juclgment of, relation of binoculiur vision to, 203

Double and single images, 20:3

Drop method of registering flow of urinc, 221

Dry cell, construction and action, 25

Dynamic and static senses, 179 sense', acuity of, 180

Dynamometer, 52

1) y'sple: 13.)

influence of, on blood-presisure, 109

LAk, diagrammatic representation of, 176 midlle, models of, 175

of $\log$-fish, dissection of, 179

Elbow, sensation of motion at, 171

Electric and chemical stimulation of temperature spots, 169

currents, types, 28

stimulation of muscle, 23

Electrodes, non-polarizable, 29

stimulating, 27,28

Electrotonus, 63

Elimination and absorption, rapidity of, 219

rapidity of, 222

Emmetropic artificial eye, ophthalmoscopic examination of, by dircet method, 194 eye, 187

Entoptic phenomena produced by tears, 192

Equilibrium a combined sense, 181

Ergograph, Mosso's, 51 spring, 52

Ergographic record of frog's gastrocnemius, 52

Esophagus, division of, 207

isolation, 207

Esthesiometer, 167

Ether and chloroform, action on heart, 90

Excised heart, 82

Excitation wave of heart, 1 ransmission of, 81

Excretion, 221, 223, 225
Exereise, effect of, on blood-pressure, 12S

Experimental jaundice, 216

Extensibility of muscle, 36

Ext risystole, s1

Eyc, anctropic, 188, 189

disisection of, $18: 3$

('rumetrop)ic, 157

artificial, ophthalmoscopie examina-

tion of, hy direct mothorl, 191

near and far points of, 193

lyes, associated movements of, 200

FAr and near points of eyc, 19:3

Fin-sightedness, 188

Fatiguc, aurlitory, 174 inusele, 49

Fibrillation, 93

Ficlal of vision, 190

Fields for colored objects, 191

lish, respiratory movenuents, 146;

Fistula, gastric, 218

Fleischl's hemoglobinometer, 74

Formation of image upon retiua, 184

Friction key, 27

Frog, gastrocnemius of, ergographic record, 52

heart of, action emrent of, 60

otolithic cavity in, 181

rheoscopic, 59

rotation effects in, 181

Function of roots of spinal cord, 163

Fusion, binocular, of dissimilar images, 203

of colors, 201

of gray and white, 201

of inuscle contraction, 42

Galvanic current, 29

Galvani's experiment, 59

Gastric fistula, 218

Gastrocnemius, frog's, ergographic record of, 52

Gastro-enterostomy, 214

Glossopharyngeal nerves, 141

Glucose, action of, 221

Gower's hemoglobinometer, 75

Gravity, center of, 39

Gray and white, fusion, 201

HeAring, 173

compound tones, 175

location of tones, 174

threshold value of sounds, 174

Heart, acceleration of, 88

action, arterial pulse and, relation between, 129

of ether and chloroform on, 90

of muscarin on, $\mathrm{S7}$

of nicotin on, 87

activities, respiratory activities and, relation between, 130

apex-bcat of, 95 
Heart dulness, area of, 95

effects of constant current on, 89 excised, 82

excitation wave, transmission of, 81

exposed, inhibition of, 114

frog's, action current of, 60

impulse, 95

inhibition of, 85

reflex, 88

isolated segments, 82

mammalian, beating, in situ, 91

dissection of, 90

rate of, 95

sounds, 97

staircase phenomenon, 89

Stannius' experiment, 89

stimulation of, 85

summation of stimuli, 89

ventricle of, isolated strips, 82

Heart-beat, conduction through ventricle, 83

effect of temperature on, 81

normal, 79

refractory period, 81

registration of, 79

Heart-block, 93

Hemin crystals, 78

Hemocytometer, 71

Hemoglobin percentage, estimation of, 73

Hemoglobinometer, Fleischl's, 75

Gowers', 75

Hemorrhage, 71

and saline injection, effect of, on bloodpressure, 111

influence of, on blood-pressure, 108

Hot and cold spots, mechanical stimulation of, 169

thermal stimulation of, 169

Hypermetropia, 188

Hypogastric nerves, function of, 225

ILLUSIONS, optical, 204

relating to weight, 170

thermal, 170

Images, dissimilar, binocular fusion of, 203

Indirect and direct vision, 200

method for ophthalmoscopic examination of artificial eye, 196

Induction coil, 29

Inductorium, 30

Inflammation of lung, phenomena of, 147 phenomena of, 147

Inhibition of heart, 85 reflex, 88

of reflexes upon central paths, 157

Innervation of bladder, 225

Insufficiency, aortic, schema of, 602 mitral, schema of, 102

Intensity of light and quality of color, 202

Intestinal juice, secretion of, chemical stimulation of, 218

into excised loops, 219

mucosa, preparations of, histologic examination of, 219
Intestine, influence of adrenal extract on, 219

osmotic power of, 217

Intracardiac blood-pressure, 115

Intrapleural pressure, 136

Intrathoracic pressure, effect of variations in, on blood-flow through lungs, 147

Intraventricular pressure, schema of, 102

Irradiation, 203

Irritability of muscle, independent, 35 of nerve, 36

Isolation of esophagus, 207

of secretory nerves, 209

Isometric myograms, 34

Isotonic myograms, 34

JAUNDICE, experimental, 216

KEY, simple, 26

Kidney, dissection of region of, 222 vasomotor supply of, 123

\section{LACTEALS, 215}

Laryngeal nerve, inferior, 143 superior, 142

Laryngoscope, 176

Larynx, 141 interior of, in man, observation of, 176

Law, all-or-none, 82

Lens, changes in shape of, 184 wabbling of, 186

Levers, systems, 38

Light, intensity of, and quality of color, 202

reaction to, 165

reflex, 184

Localization, cerebral, 163 touch, 167

Loring's ophthalmoscope, 195

Lungs, blood-flow through, effect of variations in intrathoracic pressure on, 147

collapse of, 135,136

effect of decreased atmospheric pressure on, 148

excised, 137

frog's, capillary circulation in, 147 circulation in, 147

inflammation of, phenomena of, 147

Mammalian heart, beating, in situ, 91 dissection of, 90

Mammals, rotation effects in, 180

Man, speed of nerve impulse in, 56 vasomotor phenomena in, 123

Manometer, membrane, 115

Marey's pneumograph, 129 tambour, 91

Mechanical stimulation of hot and cold spots, 169 
Mechanical stimulation of muscle, 23 of retina, 190

Medicolegal tests for blood, 77

Membrana tympani in man, observation of, 175

Membrane manometer, 115

Mercury key, 26

Metric weight, 229 exact equivalent, 230

Microscopic examination of blood, 73

Mitral insufficiency, schema, 102 stenosis, schema of, 102

Morse key, 26

Mosso's ergograph, 51

Motion, ameboid, 21 ciliary, 21 sensation of, at elbow, 171

Motor nerve, irritability near center, 59 points, stimulation of, 61

Movement, touch sensations modified by, 169 simultaneous, 170

Müller-Lyer figures, 205

Muscarin, action on heart, 87

Muscle, 21

action current, 59

contraction, compound, 43

effect of excessive stimulation on, 49 of veratrin on, 49

on volume, 38

fusion of, 42

height of, relation of load to, 44 of strength of stimulus to, 43

influence of blood-supply on, 52 of chemicals on, 48

of temperature changes on, 47

method of registering, 33

of human, 51

paradoxic, 60

single, 41

summation, 42

cross-section, relation of force to, 38

extensibility of, 36

fatigue, 49

human, law of unipolar stimulation, 65

independent irritability, 35

long and compact, comparison between, 37

manner of attachment to bones, 39

movement, 22

power, measurement, 37

smooth, 53

stimulation of, 31

direct, 23,32

indirect, 23, 32

stimuli, 22

relation of strength of, to height of contraction, 43

subminimal summation of, 44

tetanus, 43

tissuc, structure of different types, 21

tonus, 160

twitch, 41

warmer, 47

work, 45
Musele work, addition of, 46

Muscular and cutaneous sensations, 167

Myograms, isometric, 34

isotonic, 34

Myography, 25, 32

records of, fixation, 34

Myopia, 188

Near and far points of eye, 193 point, 188

Near-sightedness, 188

Negative after-images, 201

Nerve, 21

action current of, 60

anodic excitation, 64

cathodic excitation, 64

chorda tympani, function of, 210

conductivity of, 36

currents, 61

glossopharyngeal, 141

human, law of unipolar stimulation, 6.5

hypogastric, function of, 225

impulse, speed of, in cold-blooded animals, 55

in $\operatorname{man}, 56$

influence of temperature on, 59

irritability of, 36

motor, irritability near center, 59

phrenic, 137

secretory, isolation of, 209

stimulation of, 31

direct, 32

indirect, 32

superior laryngeal; 142

supply of submaxillary gland, schema illustrating, 209

sweat, 223

sympathetic, function of, 210

tissue, histulogic study, 55

trigeminal, 141

vagus, influence of, 207

main trunk, 143

Nerve-fibers, conduction in both directions by, 57

Nervous regulation of respiration, 141 system, 163, 165

frog's dissection of, 155

localization of function in, 156

reflex action, 155

Neurons, histologic study, 155

Nicotin, action on heart, 87

Non-polarizable electrodes, 29

Nystagmus, railroad, 181

OBSERVATION of interior of larynx in man, 176

of membrana tympani in man, 175

Olfactory cells, distribution of, 174

structure of, 174

fatigue, 174

latency, 174

qualitative changes before exhaustion, 
Ophthalmometer, 193

Ophthalmoscope, Loring's, 195

Ophthalmoscopic examination of ametropic artificial eye by direct method, 195

of artificial eye by indirect method, 196

of emmetropic artificial eye by direct method, 194

Optical illusions, 204

Osmometer, 217

Osmosis, 217

Osmotic power of intestine, 217

Otolithic cavity in frog, 181

PaIn spots, 170

Pancreas, position of lucts of, diagram showing, 213

Pancreatic juice, collection of, 213

Paradoxic contraction of muscle, 60 resistance, 171

Patellar reflex time, 166

Percussion, 139 of human stomach, 208

Perimeter, 191

Peristalsis, 215, 218 influence of salts on, $21 \mathrm{~s}$

Phenomenon, Babinski, eliciting of, 160 Purkinje's, 202

Phlebograph, use of, 129

Phosphenes, 190

Photic stimulation of musele, 23

Phrenic nerves, 137

Pilomotor reactions, 225

Pithing, 23

Placenta, study of, 146

Plasma, blood, relative amounts, 70

Plethysmograph, cardiac, 92

Pneumograph, Marey's, 129

Pohl's commutator, 56

Polarization, 28

Polygraphy, 129

Positive after-images, 200

Posture, effect of, on blood-pressure, 127 influence of, on blood-pressure, 107

Preparation of secretin, 213

Pressure and touch, peculiar phenomena and illusions of, 168

in tympanum, 175

intrapleural, 136

intraventricular, schema of, 102 secretory, 210

Primary current, 29

Protoplasmic streaming, 21

Pulmotor, 140

Pulse, arterial, character and velocity, 129 heart action and, relation between, 129

cause and velocity, schema illustrating, 103

schema of, 102

venous, character and velocity, 129

Pupil, size of, changes in, 184

Purkinje's phenomenon, 202 QUALITY of color and intensity of light,
202

Quickly interrupted current, 29

RAILROAD nystagmus, 181

Rapidity of absorption and elimination, 219

of elimination, 222

Reaction time, 165 with choice, 166

to light, 165

to sound, 166

to touch, 165 sensation, 165

Reactions, pilomotor, 225

Red blood cells, human, counting of, 73 corpuscles, enumeration, 70 simultaneous count, 73

Reflex, accommondation, 184 action, 159 study of, 155

centers for hind legs, localization of, 156

light, 184

time, 157 patellar, 166

winking time, 166

Reflexes, action, 159

- exaggeration of, by means of strychnin, 15 )

in man, 159

inhibition of, by higher centers, 159 upon central paths, 157

spreading of, 157

tendon, in man, 160

Relation of weight to area stimulated, 170

Resistance, paradoxic, 171

Respiration, 133

accessory movements, 141

action of thorax in, 134

artificial, 140

center of, localization, 145

elimination of carbon dioxid in, 151 of water in, 151

forced, 135

frequency, 139

glossopharyngeal nerve in, 141

inferior laryngeal norve in, 143

influence of carbon dioxid in, 140

larynx in, 141

main trunk of vagus nerve in, 143

meehanies of, 1333

nervous regulation of, 141

normal, 134

placenta in, 146

self-regulation, 141

superior laryngeal nerve in, 142

trigeminal nerve in, 141

Respiratory activities, heart activities and, relation between, 130 movements in fish, 146

Retina, formation of image upon, 184 mechanical stimulation of, 190

Retinal blood-vessels, 199

image, formation of, 186 
Rheoscopic frog, 59

Riva-Rocei's sphygmomanometer, 12.;

Roots of spinal cord, function of, 16 ij:3

Rotation effects in frog, 181 in mammals, 180

Rotatory apparatus for color disks, 2)1

Saline injection and hemorrhage, effect of, on blood-pressure, 111

Saliva, secretion of, 209

Salivary glands, disseetion of, 211

Salts, influence of, on peristalsis, 218

Scheiner's experiment, 186

Sciatic nerve, vasomotor aetion of, 118 , 119

Secondary current, 29

Secretin, action of, 214 preparation of, 213

Secretion by bloodless gland, 210 norinal, of urine, 221

of bile, 215

of intestiual juice into excised loops, 21!) of saliva, 209

of sweat, 223

Secretory nerves, isolation of, 209 pressure, 210

Semicircular canals, action of, model illustrating, 180

position of, 179

Sensation of motion at elbow, 171

Sense oryans, 167, 173

Sharlow test, 196

Shape of lens, changes in, 184

Simultaneous movements, 170

Single and double images, 20:3

Size of pupil, changes in, 184

Skiaseopy, 196

Smell, 173

Smooth muscle, 53

Snellen's test types, 194

Sodium chlorid, action of, 221

Solidity, judgments of, relation of binocular vision to, 203

Sounds, reaction to, 166 threshold value of, 174

Speeific gravity of blood, 75

Spectroseopic examination of blood, 77

Spheric aberration, 190

Sphygmograph, application of, 129 Didgeon, 129

Sphygmomanometer, application of, 125

Sphygmotonometer, 125

Spinal cord, roots of, function of, 163

Spirometer, 133

Splanehnic nerve, greater, vasomotor aetion of, 121

Spot, blind, 199 contours of, 199 pain, 170

yellow, 199

Spring ergograph, 52

Staircase phenomenon, 89

Stannius' experiment, 89

Static and dynamic senses, 179
Stenosis, aortic, schema of, 102

mitral, schema of, $10^{\circ} 2$

Stethograph, Marey's, 130, 131

Stethography, 139

Stimulating electrodes, $27,2 \mathrm{~S}$

Stimulation, excessive, offect on musclo contraction, 49

of cerebrum, 163

of muscle, 31

direct, 32

indirect, 32

of nerve, 31

direct, 32

indirect, 32

threshold of, 160

Stimulus, inadequate, 173

muscle, relation of strength of, to heiglit of contraction, 43 subminimal, summation of, 44 thermal, effect of, 157

Stomach contents, 216

human, percussion of, 208

itroaming, protoplasmic, 21

itrychnin, exaggeration of reflexes by means of, 159

N'uhmaxillary gland, nerve supply of, schema illustrating, 209

vasomotor changes in, produced by stimulation of chorda tympani and sympathetic nerve, 210

Niweat nerves, 223

secretion of, 223

Siveating in man, $22: 3$

S'wim test, 137

Sympathetic nerve, cervical, visomotor action, 117

function of, 210

TACTILE corpuscles, histologic examination of, 167

'Tambour, Marey's, 91

Taste, 173

distribution of, 173

electric stimulation, 173

elimination of sweet and bitter, 174

inadequate stimuli, 173

reaction to single papilla, 173

threshold value of, 173

Taste-buds, structure of, 173

Tears, entoptic phenomena produced by, 192

Temperature, after-image of, 169

body, 223

changes, influenee on muscle contraction, 47

eontrast, 170

effect of, on heart-beat, 80 .

influence of, on nerve, 59

sense, acuity of, 170

spots, chemical and electric stimulation of, 169

Tendon reflexes in man, 160

Test, swim, 137

types, Snellen's, 194 
Tetanic current, 29

Tetanus, incomplete, of heart, 89 muscle, 43

Thermal illusions, 170

stimulation of hot and cold spots, 169 of muscle, 23

stimuli, effect of, 157

Thoma-Zeiss hemocytometer, 71

Thoracic duct, 216

Thorax in respiration, action of, 134

Tones, compound, 175

location of, 174

Tonus, muscle, 160

Touch and pressure, peculiar phenomena and illusions of, 168

discrimination, 167

localization, 167

projection of sensation of, 169

reaction to, 165

sensations, adaptation to, 168

modified by movement, 169 reaction to, 165

Traube-Hering curves, 106

Trigeminal nerves, 141

Twitch, muscle, 41

Tympanum, pressure in, 175

Types, test, Snellen's, 194

UNIPOLAR stimulation of human muscle and nerve, law of, 65

Urine, flow of, drop method of registering, 221

normal secretion of, 221

VAGI nerves, both, division of, effect of, on blood-pressure, 114

influence of, 207

intact, stimulation of, effect on bloodpressure, 113

Vagus nerve, one, division of, effect on blood-pressure, 114

main trunk, 143

Valves, venous, position and function, 109

Vasomotor action of cervical sympathetic nerve, 117

of depressor nerve, 118

of greater splanchnic nerve, 121

of sciatic nerve, 118, 119
Vasomotor changes in submaxillary gland produced by stimulation of chorda tympani and sympathetic nerve, 210 phenomena in man, 123 supply of kidney, 123

Venous blood, 68 blood-pressure, 128 pulse, character and velocity, 129 valves, position and function, 109

Ventricle of heart, conduction of heartbeat through, 83 isolated strips, 82

Veratrin, effect on muscle contraction, 49

Vision, 183, 189, 193, 199

binocular, relation of, to judgments of direction, 203 of distance, 203 of solidity, 203

field of, 190

Visual angle, 194 axes, convergence of, influence of, 203

Vocal cords, abduction of, 143 and adduction of, 143

WABBLing of lens, 186

Warmer, muscle, 47

Water, elimination of, in respiration, 151

Wave of deglutition, 207

Weber's law, 170

Weight, apothecaries', 229 exact equivalent, 230

discrimination of, Weber's law, 170

illusions relating to, 170

metric, 229 exact equivalent, 230

relation of, to area stimulated, 170

Weights and measures, 229

White and gray, fusion of, 201 blood corpuscles, enumeration of, 71

Winking time, reflex,

Wintrich's modification of Hutchinson's spirometer, 133

Work, muscular, 45 addition of, 46

Work-adder, 45

YELLOW spot, 199

ZolLner's lines, 204 



UNIVERSITY OF CALIFORNIA

MEDICAL CENTER LIBRARY

THIS BOOK IS DUE ON THE IAST DATE STAMPED BELOW

Books not returned on time are subject to a fine of $50 \mathrm{c}$ per volume after the third day overdue, increasing to

$\$ 1.00$ per volume after the sixth day. Books not in de. mand may be renewed if application is made before expiration of loan period.

\section{Fto 161942}

\section{MAR 141942}




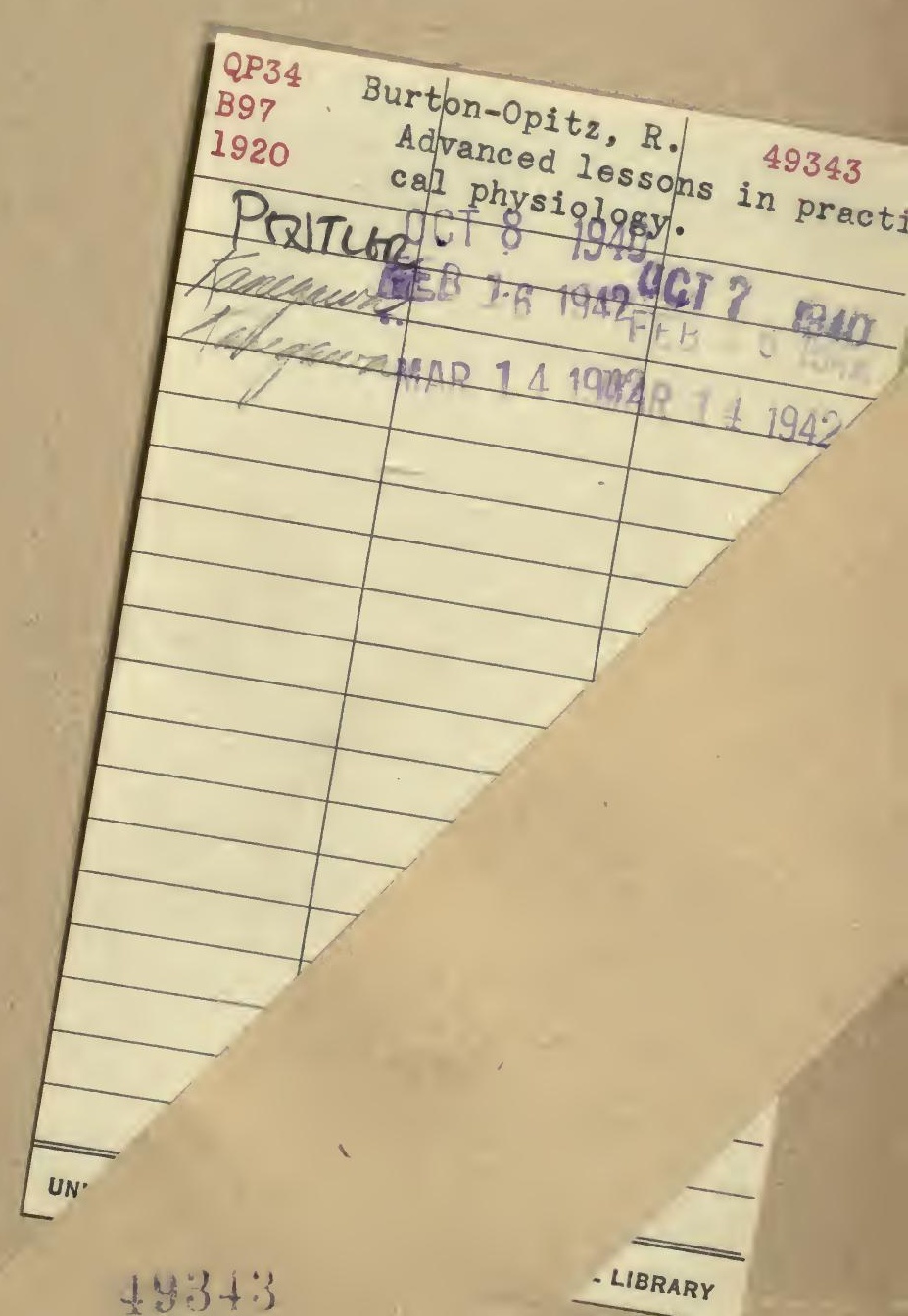


